Aus der Klinik und Poliklinik für Frauenheilkunde und Geburtshilfe Innenstadt

Klinikum der Ludwig-Maximilians-Universität München

Direktor: Prof. Dr. med. habil. Klaus Friese

\title{
Reiten in der Schwangerschaft
}

\author{
Dissertation \\ zum Erwerb des Doktorgrades der Medizin \\ an der Medizinischen Fakultät der \\ Ludwig-Maximilians-Universität zu München \\ vorgelegt von \\ Susanna Kramarz
}

aus

Leipzig

2011 


\section{Mit Genehmigung der Medizinischen Fakultät} der Universität München

Berichterstatter:

Mitberichterstatterin:

Mitbetreuung durch den promovierten Mitarbeiter:

Dekan:

Tag der mündlichen Prüfung:
Prof. Dr. med. habil. Klaus Friese Universitäts-Frauenklinik

Prof. Dr. Dr. Angela Schuh

Privatdozent Dr. med. Ioannis Mylonas

Prof. Dr. med. Dr. h.c. M. Reiser, FACR, FRCR

07.04 .2011 


\section{Inhaltsverzeichnis}

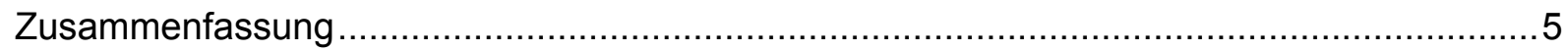

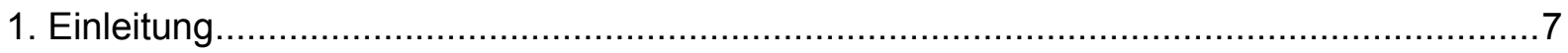

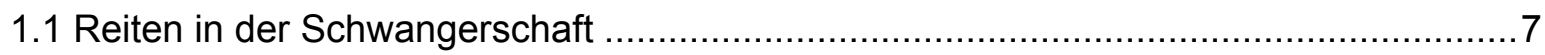

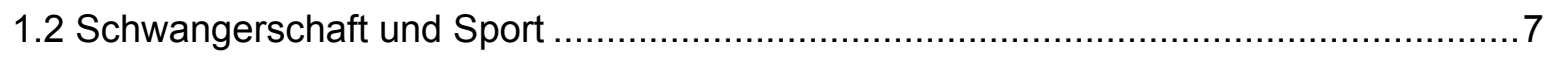

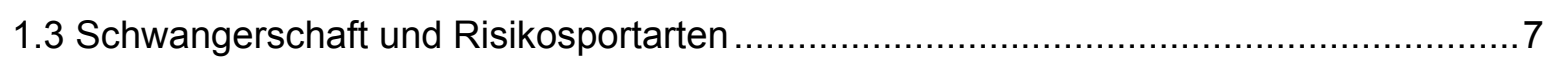

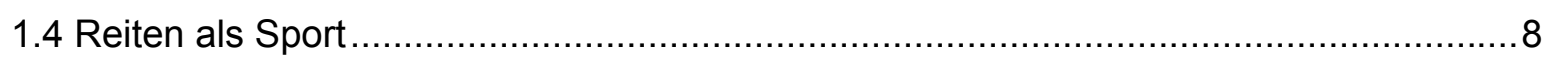

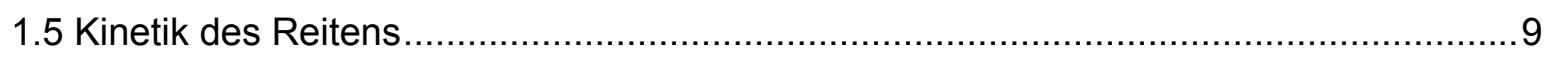

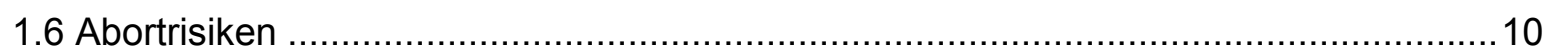

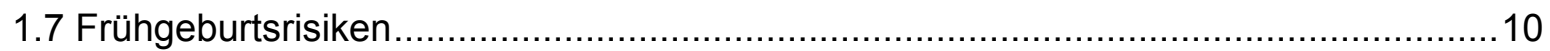

1.8 Sportliches Training, Beckenboden und protrahierter Geburtsverlauf........................11

1.9 Kindslage - externe Einflüsse und kindliches Outcome bei Beckenendlage ..............11

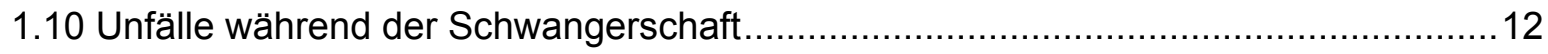

1.11 Unfälle beim Reiten und beim Umgang mit dem Pferd ......................................12

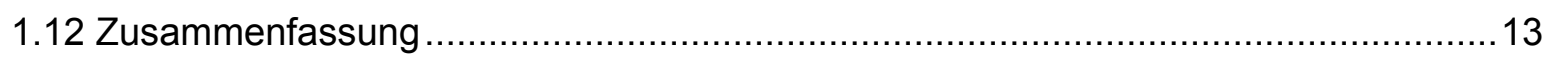

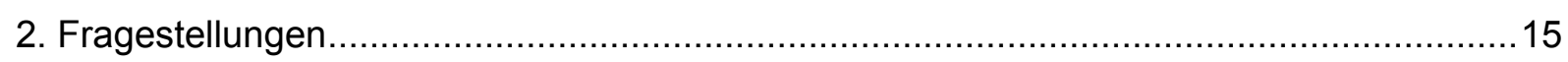

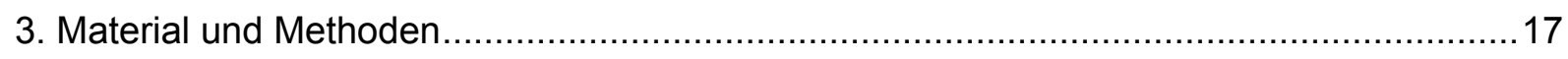

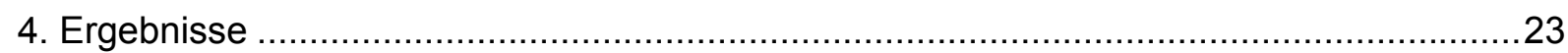

4.1 Vergleich mit der Niedersächsischen Perinatalstudie (Normalkollektiv)......................23

4.2 Ermittlung des Frühgeburtsrisikos bei Reiterinnen nach Gewichtung ........................26

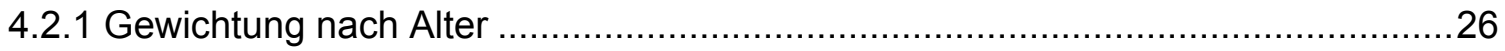

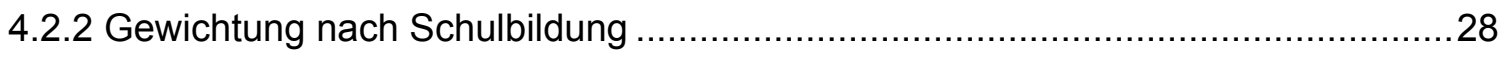

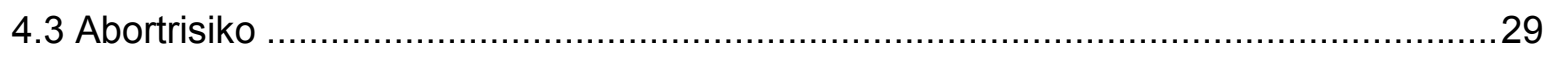

4.4 Einfluss unterschiedlicher Reitweisen auf die Frühgeburtlichkeit.............................. 31

4.4.1 Weiterreiten während der Schwangerschaft - inverse Korrelation zur

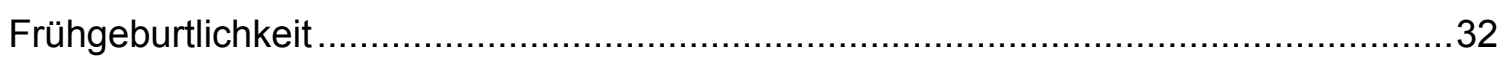

4.5 Weitere Risiken für Frühgeburtlichkeit.......................................................... 35

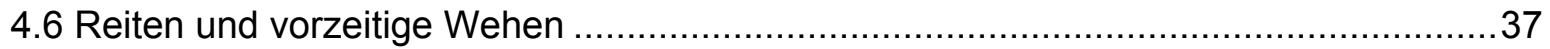

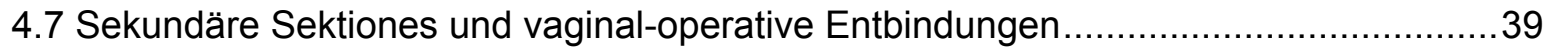

4.8 Vaginal-operative Entbindungen in Abhängigkeit von der reiterlichen Aktivität............42

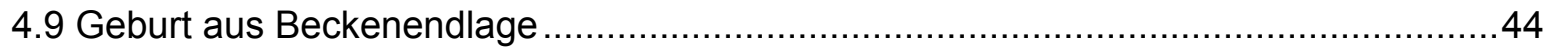

4.9.1 Schädellage vs. Beckenend- und Querlage - Häufigkeiten .................................44

4.9.2 Vergleich Entbindungen aus Schädellage vs. Beckenend- und Querlage .............45

4.9.3 Schädellage vs. Beckenend- und Querlage - weitere Unterschiede ....................45

4.9.4 Schädellage vs. Beckenend- und Querlage - nicht unterschiedliche Parameter .. 46

4.9.5 Kindslage beim Reiten bis zur Entbindung am Termin.......................................46 
4.10 Unfälle beim Reiten während der Schwangerschaft............................................48

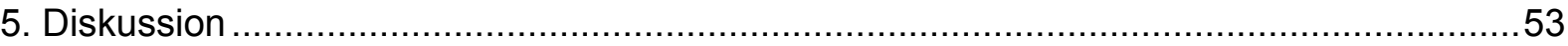

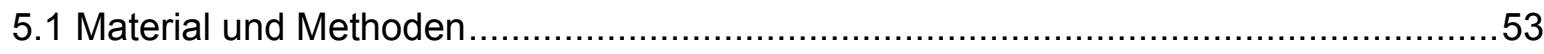

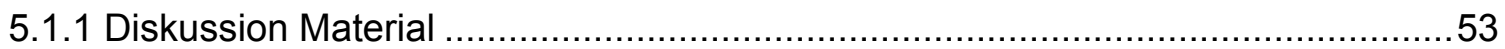

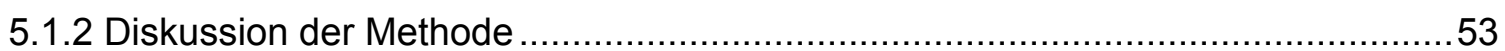

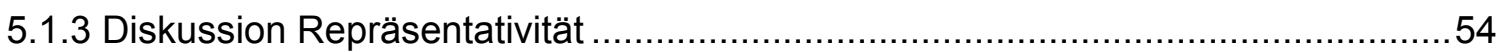

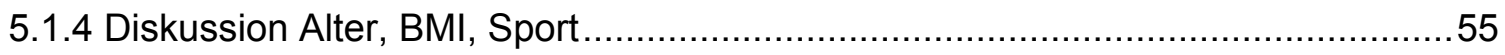

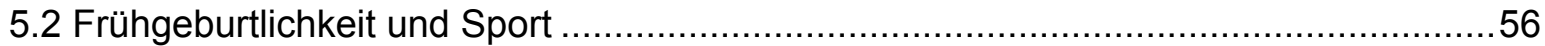

5.3 Körperliche Belastung und körperliche Arbeit während der Schwangerschaft............59

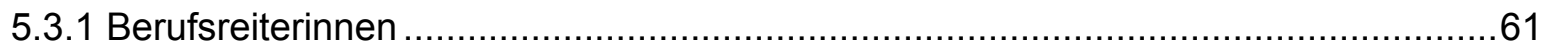

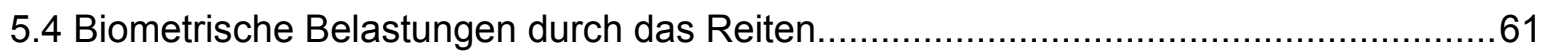

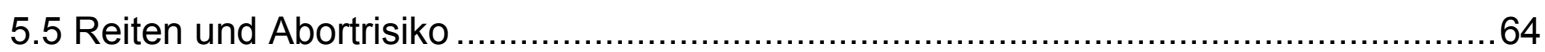

5.6 Beckenboden und Reiten in der Schwangerschaft..............................................67

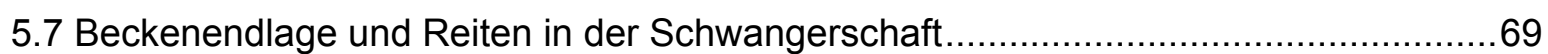

5.8 Unfälle durch Reiten und Umgang mit dem Pferd in der Schwangerschaft .................70

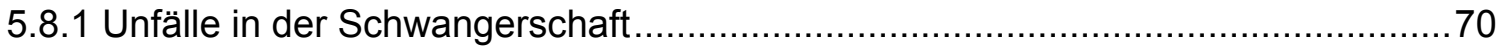

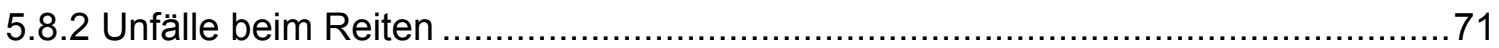

5.8.3 Unfälle beim Umgang mit dem Pferd........................................................... 72

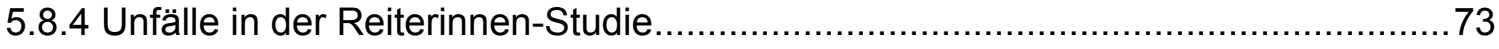

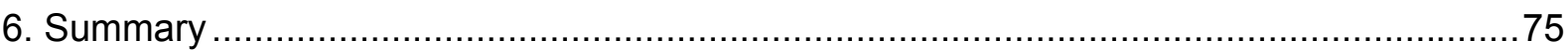

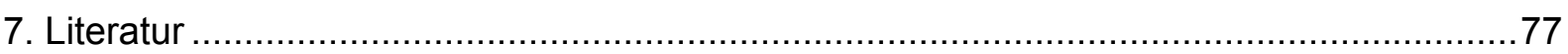

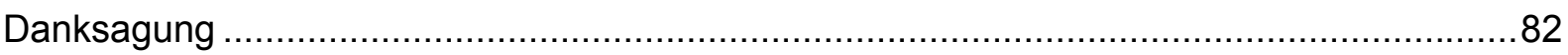

Curriculum vitae

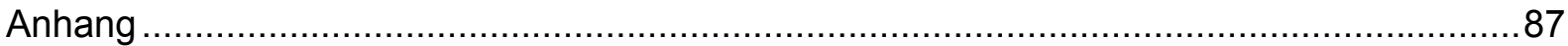

Vorabpublikationen

Reiten in der Schwangerschaft - der Fragebogen

Tabellenband

Tab. A1 Primiparae, ausführliche Dokumentation

Tab. A2 Primiparae, verdichtet

Tab. A3 Aborte, ausführliche Dokumentation

Tab. A4 Primiparae, Teilnehmerinnen mit vorzeitigen Wehen, verdichtet

Tab. A5 Primiparae, Entbindungen aus Beckenendlage, verdichtet

Tab. A6 Geritten bis zur Entbindung in Terminnähe, BEL vs. SL, verdichtet

Tab. A7A Primiparae - Spontanentbindungen vs. vaginal-operative Entbindungen

Tab. A7B Primiparae - Spontanentbindungen vs. sekundäre Sectiones

Tab. A8 Unfälle beim Reiten und beim Umgang mit dem Pferd, verdichtet 


\section{Zusammenfassung}

Die Auswertung einer retrospektiven, Internet-basierten Befragung von 1851 Reiterinnen zeigt keine Anhaltspunkte dafür, dass Reiten während der Schwangerschaft zu einer erhöhten Rate an Aborten oder Fehlgeburten führt.

Auch eine erhöhte Neigung zur Frühgeburtlichkeit kann nach Gewichtung der Daten beim Vergleich mit den Daten der Niedersächsischen Perinatalstudie nicht festgestellt werden.

Beim Vergleich von Reiterinnen mit Entbindung am Termin und mit Frühgeburt lässt sich keinerlei Hinweis darauf finden, dass bei gesunden Schwangeren bestimmte reiterliche Disziplinen oder die Intensität und Dauer des Reitens während der Schwangerschaft einen Einfluss auf die Dauer der Schwangerschaft haben.

Gegenüber den Vergleichsdaten der Niedersächsischen Perinatalstudie wird keine Zunahme an vaginal-operativen Entbindungen oder sekundären Sectiones gefunden.

Jede elfte Teilnehmerin hatte in der Schwangerschaft einen oder mehrere Unfälle beim Reiten oder beim Umgang mit dem Pferd. Besonders gefährdet sind ambitionierte und professionelle Reiterinnen, Reiterinnen im gehobenen Springsport und Reiterinnen, die erhöhte Stress-Parameter angeben. Aber auch bei schwangeren Reiterinnen ohne diese Risikofaktoren ereignen sich Unfälle beim Reiten oder beim Umgang mit dem Pferd. Das Unfallrisiko kann durch keine Maßnahme sicher ausgeschlossen werden.. 


\section{Einleitung}

\subsection{Reiten in der Schwangerschaft}

Zwischen 5.000 und 10.000 Reiterinnen ${ }^{1}$ werden jährlich in Deutschland schwanger. Viele von ihnen haben ein eigenes Pferd, etliche sind Berufsreiterinnen. Sie selbst, ihre Ärzte, Trainer und bei Berufsreiterinnen auch die Arbeitgeber können sich für die Überlegung, ob und wie lange eine Reiterin in der Schwangerschaft im Sattel bleiben kann, bisher nur auf Vermutungen stützen: Zum Thema „Reiten in der Schwangerschaft" wurden weltweit bislang keine Untersuchungen publiziert. Diese Feststellung war der Anlass, die hier vorgelegte Studie durchzuführen.

\subsection{Schwangerschaft und Sport}

Sport zu treiben wird Schwangeren heute im Allgemeinen empfohlen, wenn nicht gesundheitliche Gründe dagegen stehen. Dabei soll die sportliche Betätigung möglichst im gemäBigten Ausdauer- und Wellness-Bereich stattfinden (Huch 2005, Schlüssel 2008). Allerdings zeigen Untersuchungen an Leistungssportlerinnen, dass es durchaus möglich ist, bis weit in die Schwangerschaft hinein einen beträchtlichen Teil des Trainingspensums beizubehalten (Kardel 2005, Pettinen 2007).

Die körperliche Belastbarkeit der Schwangeren vor allem im Hinblick auf Spätschwangerschaft und Geburt profitiert von einem angemessenen sportlichen Trainingszustand (AGOC 2002). Das Schmerzerleben unter der Geburt wird von Sportlerinnen als weniger belastend eingeschätzt als von Frauen, die während der Schwangerschaft körperlich inaktiv waren (Reimers 2008). Der Fet wird durch die erhöhte Herzfrequenz und Muskeltätigkeit der Mutter nicht beeinträchtigt.

\subsection{Schwangerschaft und Risikosportarten}

Schwangeren wird davon abgeraten, während der Schwangerschaft Sportarten weiter auszuüben, in denen schnelle Bewegungen, Drehungen und Stürze unvermeidlich sind, wie Mannschaftssportarten, Eiskunstlauf, Kampfsportarten, Alpinski und auch Reiten (Paringer 2010). Dieser Rat wird unter der Vorstellung erteilt, dass schnelle Bewegungen und Drehungen eine mechanische Belastung für Plazenta, Nabelschnur, Amnion und Embryo bzw. Fet darstellen und zu Plazentaablösungen, Nabelschnurumschlingungen und vorzeitigem

\footnotetext{
${ }^{1}$ Die Zahl beruht auf Hochrechnungen; Grundlage sind die Bevölkerungsstatistik des Bundesamts für Statistik, die Statistik der Deutschen Reiterlichen Vereinigung und Auswertungen der BabyCare-Studie, siehe S. 71 ff.
} 
Blasensprung führen könnten. Obwohl anzunehmen ist, dass viele Schwangere ihre gewohnten Sportarten - auch Risikosportarten - zumindest noch eine Zeitlang weiterführen, wurde bisher nicht systematisch untersucht, ob diese Befürchtung den Tatsachen entspricht. Reiten hat in dieser Liste eine Sonderstellung. Denn beim Reiten sind dynamische Bewegungen und Sprünge, Drehungen um die Körperachse oder Stürze nicht als regelmäßiger Bestandteil der Sportart vorgesehen.

\subsection{Reiten als Sport}

Reiten besteht aus sportphysiologischer Sicht zu 45\% aus Koordination, zu 30\% aus Beweglichkeit und nur zu 25\% aus Kraft, Ausdauer, Schnelligkeit (Heipertz-Hengst 2002). Eine gute Koordination ist die wesentliche Voraussetzung für gutes Reiten; eine fehlende Koordination verhindert die Anpassung an die Bewegungen des Pferdes, der Sitz des Reiters wird unbequem und unsicher, das Pferd verspannt sich, die Unfallgefahr steigt. Beweglichkeit und Reaktionsschnelligkeit sind zum Reiten ebenfalls als Grundlage erforderlich, werden aber nur beim Western-, Gelände-, Spring- und Rennreiten in höherem Maß beansprucht. Ein Training bis an den Rand der physischen Leistungsfähigkeit ist beim Reiten nicht erwünscht: Aus Sicherheitsgründen brauchen Reiter immer Reserven, um das Pferd im Griff zu behalten und auf unerwartete Situationen entschieden reagieren zu können.

Das Ziel des reiterlichen Sports ist nicht eine maximale physische Leistungsfähigkeit des Reiters, sondern Übereinstimmung zwischen Reiter und Pferd. Ist dank jahrzehntelanger Erfahrung diese Übereinstimmung erreicht, dann ist es anders als bei allen anderen Sportarten möglich, fast bis ins Seniorenalter Spitzenleistungen zu erbringen. Auch während der Schwangerschaft ist es bei entsprechender vorheriger Ausbildung möglich, im Spitzensport mitzureiten. Reiterlicher Leistungssport in der Schwangerschaft ist damit für die vorliegende Untersuchung nicht unter dem Aspekt des Trainings an der körperlichen Leistungsgrenze relevant. Wichtig sind hier vier andere Gesichtspunkte:

- Die Tatsache, dass beim Reiten erhebliche Schwingungsbelastungen auf die Schwangere wirken,

- dass das Reiten und die Arbeit mit dem Pferd mit hohen Trainingszeiten einhergehen kann,

- dass die körperliche Anstrengung bei der Stallarbeit mit berücksichtigt werden muss, und

- dass der Umgang mit dem Pferd nie als völlig sicher gelten kann. 


\subsection{Kinetik des Reitens}

Untersuchungen zu Schwingungsbelastungen beim Reiten, durchgeführt auf einem standardisierten Hochgeschwindigkeits-Laufband für Pferde, haben gezeigt, dass auf die Wirbelsäule von Reitern im Trab und Galopp Schwingungsbelastungen (horizontale Beschleunigung) wirken, die vor allem bei entsprechender Disposition geeignet sind, dauerhafte Schäden der Wirbelsäule zu verursachen (Berufsgenossenschaftliches Institut für Arbeitssicherheit 1999).

Die Untersucher kommentierten ihre Ergebnisse und kamen zu der Schlussfolgerung, dass eine gut trainierte Becken- und Rumpfmuskulatur die Belastungen zum großen Teil kompensieren könne.

Diese Haltung ist inwischen umstritten. In einer neueren Untersuchung von Berufsreitern (Kraft 2009) fand man bei $88 \%$ der Dressurreiter (mittleres Alter 33,5 Jahre) Degenerationserscheinungen in den Bandscheiben der Lumbal- und Kreuzbeinwirbel, die im Magnetresonanztomogramm verifizierbar waren, nicht jedoch bei Springreitern. Bei den Springreitern hatten die häufig berichteten Rückenschmerzen eher ihre Ursache in einer chronischen muskulären Dysbalance durch die Dauerbelastung.

Die verifizierbaren Verschleißerscheinungen bei Dressurreitern geben einen weiteren Hinweis darauf, dass reiterliche Dauerbelastung eine unphysiologische Belastung darstellen und organische Schäden hervorrufen kann.

Dass Dressurreiter stärker belastet sind als Springreiter, ist gut erklärbar. Bei allen Fragen zur biometrischen Belastung von Rumpf und Wirbelsäule beim Reiten muss zwischen zwei grundsätzlich unterschiedlichen Reitweisen unterschieden werden: Dressurreiten im so genannten „tiefen Sitz“ bedeutet, mit langen Steigbügeln fest im Sattel zu sitzen und alle Bewegungen des Pferdes möglichst direkt mitzureiten. Auf diese Weise hat der Reiter mit seinem Sitz eine intensive Einwirkung auf das Pferd. Dieser Reitstil ist mit einer dauerhaften biomechanischen Schwung- und Stoßbelastung verbunden, weil einerseits jede Bewegung, Anspannung und Gewichtsverlagerung des Reiters direkt auf den Pferderücken übertragen wird, andererseits aber jede Bewegung des Pferdes ebenso direkt auf Becken, Rücken und Rumpf des Reiters wirkt und mitgeritten wird.

Beim Reiten im „leichten Sitz“ dagegen steht der Reiter in den Bügeln und federt alle Bewegungen des Pferdes mit der Oberschenkel- und Hüftmuskulatur ab. Die Rumpfmuskulatur 
wird zusätzlich zur Festigung eingesetzt. Auch das „Leichttraben“, bei dem jeder zweite Trab-Tritt des Pferdes im Sattel gesessen, jeder zweite in den Steigbügeln gestanden wird, ist bei geübten Reitern eine angenehme Technik, um die Stoßbelastungen für Rumpf und Rücken weitgehend zu reduzieren. Der „leichte Sitz“ ist anstrengend und braucht einen guten Trainingsstand. Aber er ermöglicht es, Stoßbelastungen für Wirbelsäule und Rumpf fast vollständig auszuschalten (siehe S. 58, Kapitel 5.3).

Inwieweit unterschiedliche Arten des Reitens - tiefer Dressursitz oder leichter Sitz - einen Einfluss auf den Verlauf einer Schwangerschaft haben, wurde bisher nicht untersucht.

\subsection{Abortrisiken}

Genaue Zahlen zur Aborthäufigkeit existieren nicht. Es wird angenommen, dass zwischen $10 \%$ und $15 \%$ aller Schwangerschaften in einem Abort enden (Baltzer 2006), etwa 3-5\% ab Bekanntwerden der Schwangerschaft (Madson 2007). Ursachen sind in der überwiegenden Zahl der Fälle nicht lebensfähige Fehlbildungen beim Embryo, insbesondere genetische Aberrationen, außerdem Fehlbildungen des Uterus, Infektionen, hormonelle Störungen, mütterliches Über- und Untergewicht und immunologische Faktoren. In vielen Fällen sind die Ursachen jedoch nicht bekannt.

Zur Fragestellung, ob sportliche Belastung das Abortrisiko erhöhen kann, existiert eine aktuelle Subgruppen-Analyse der Danish National Birth Cohort (Madson 2007); hier wurde bei einer Trainingsintensität von 7 Stunden pro Woche und darüber bei „High-impact“-Sportarten wie Joggen und Tennisspielen eine erhöhte Aborthäufigkeit in den ersten 18 Wochen der Schwangerschaft berichtet, beim Schwimmen eine geringere. Beim Schwangeren, die

„Radfahren oder Reiten“ im ersten Trimenon angaben, wurde eine Erhöhung der Aborthäufigkeit in nicht signifikantem Umfang gefunden. Bei geringeren Trainingszeiten wurde kein Zusammenhang zwischen dem Sport bzw. der Sportart und der Abortrate gefunden. Die Reitweise wurde in der Untersuchung nicht erfragt.

Ein Einfluss reiterlicher Aktivität auf die Frühabort-Rate wurde bisher nicht untersucht.

\subsection{Frühgeburtsrisiken}

Die derzeit am besten nachgewiesenen Frühgeburtsrisiken sind

- Frühgeburt in der Anamnese,

- aufsteigende Infektionen, 
- soziale Faktoren,

- Stress, psychische Belastung,

- Rauchen, Drogen.

Unangemessene körperliche Belastung als auslösender Faktor für vorzeitige Wehen ist seit langem bekannt (Seitz 1933). Als Risiko für Frühgeburtlichkeit gilt sie, wenn weitere Faktoren hinzukommen wie fehlende Erholungsmöglichkeiten im Tagesverlauf, schlechter Ernährungszustand, Stress, Krankheit, Nachtarbeit (Lawson 2009).

Eine hohe sportliche Belastung gehört nicht zu den bekannten Frühgeburtsrisiken. Allerdings gehen alle Untersuchungen an schwangeren Leistungssportlerinnen immanent davon aus, dass schwangere Sportlerinnen ihr Training eigenverantwortlich regulieren und mit fortschreitender Schwangerschaft reduzieren, um sich und das Kind nicht zu gefährden.

Untersuchungen, ob leistungssportlich betriebenes Reiten mit Trainingsbelastungen von $7 \mathrm{~h}$ pro Woche und mehr den Verlauf einer Schwangerschaft beeinflussen kann, existieren bisher nicht.

\subsection{Sportliches Training, Beckenboden und protrahierter Geburtsverlauf}

Druckmessungen des Beckenbodens zeigen einen hohen Innervierungs- und Aktivierungsstand der Beckenbodenmuskulatur bei Reiterinnen und Reitern (Osthoff 2008). Jedoch gibt es bisher keine Untersuchungen $\mathrm{zu}$ der Fragestellung, ob eine rigide, trainierte Beckenbodenmuskulatur einen klinisch relevanten Einfluss auf den Geburtsverlauf und das kindliche Outcome ausüben könnte.

Der Einfluss des Trainingsstandes und der Festigkeit der Beckenbodenmuskulatur auf den Geburtsverlauf bei Reiterinnen wurde noch nicht untersucht.

\subsection{Kindslage - externe Einflüsse und kindliches Outcome bei Beckenendlage}

Empirisch wird vermutet, dass Reiterinnen häufiger aus Beckenendlage entbinden als NichtReiterinnen. Dieser Überlegung liegt die Vorstellung zu Grunde, dass der Fet in utero die reiterlichen Bewegungen seiner Mutter als angenehmer empfindet, wenn er sich in Beckenlage befindet als in Schädellage. In der vorliegenden Untersuchung wird diese These überprüft. 
Ein Zusammenhang zwischen körperlichen Belastungsmustern der Schwangeren und der Kindslage ist aus der Literatur nicht bekannt: Beckenendlagen gelten als idiopathisch. In $50 \%$ handelt es sich um Primiparae. Es werden familiäre Häufungen beobachtet; zudem ist ein Zusammenhang mit Frühgeburtlichkeit, Mehrlingsschwangerschaft, Oligo- oder Hydramnion, Fehlbildungen des Kindes oder des Uterus, Nabelschnurdeviationen, Placenta praevia, Rauchen, Diabetes und erhöhte Gewichtszunahme während der Schwangerschaft bekannt (Rayl 2005).

\subsection{Unfälle während der Schwangerschaft}

Verletzungen durch Unfälle oder Gewalteinwirkung sind eine häufige und wichtige Schwangerschaftskomplikation: Weltweit - von USA über Europa bis Vorderasien - müssen 5 bis 8\% aller schwangeren Frauen wegen eines Unfalls oder einer Verletzung ärztlich behandelt werden (El Kady 2004).

Unfälle und Gewalteinwirkung stellen die häufigsten nicht-geburtshilflichen Todesursachen für Mutter und Kind in der Schwangerschaft dar (Chang 2009). Deshalb sollten Unfallrisiken in der Schwangerschaft so weit wie möglich ausgeschaltet werden.

\subsection{Unfälle beim Reiten und beim Umgang mit dem Pferd}

Unfälle beim Reiten und beim Umgang mit dem Pferd sind in den ersten Jahren der reiterlichen Ausbildung häufig. Mit zunehmender Erfahrung sinkt die Unfallgefahr. Reiten ist damit eine Hochrisiko-Sportart für Unerfahrene.

Eine zweite gefährdete Gruppe sind hoch erfahrene, trainierte Reiter, die aufgrund ihrer Expertise in der Ausbildung und Korrektur von jungen Pferden und von Problempferden eingebunden sind, Pferde in der Ausbildung und im Training für den Dressur-, Spring- und Geländesport intensiv fordern und sich mit innen auseinandersetzen und die mit Hengsten arbeiten: Anders als bei Stuten gehört das Steigen ebenso wie das Besteigen anderer Pferde bei Hengsten zum natürlichen Bewegungs- und Verhaltensrepertoire. Es erfordert erhebliches Feingefühl, Durchsetzungsvermögen und Können, diese Neigung sowohl im Umgang mit den Tieren als auch beim Reiten zu beherrschen und zu kompensieren.

Aber Unfälle und Stürze beim Reiten oder beim Umgang mit dem Pferd sind - anders als bei Mannschafts- und Kampfsportarten - kein genuiner Bestandteil des Reitsports. Wenn die reiterlichen Techniken sicher beherrscht werden, der Reiter ausreichend konzentriert und 
aufmerksam ist, das Pferd einen verlässlichen Charakter und eine solide Ausbildung hat und nicht am Rand seiner Belastungsfähigkeit geritten wird, sind Unfälle und Verletzungen nicht vorgesehen. Trotzdem ereignen sich nach einer Befragung der Bundesanstalt für Arbeitsschutz und Arbeitsmedizin (BauA 2002) jährlich in Deutschland etwa 56.000 Unfälle beim Reiten oder beim Umgang mit Pferden. Pferdewirte werden in den landwirtschaftlichen Berufsgenossenschaften zu den Berufen mit der höchsten Unfallquote gerechnet (Pferdebetrieb 2000).

Zum Unfallrisiko von Schwangeren beim Reiten und beim Umgang mit dem Pferd existieren bisher keine Untersuchungen.

\subsection{Zusammenfassung}

Mit der vorliegenden Arbeit soll der Versuch unternommen werden, die in der Einleitung aufgeführten Fragen und Problemstellungen zum Risikopotential des Reitens in der Schwangerschaft mit Hilfe einer retrospektiven Befragung an Reiterinnen zu beantworten, deren Schwangerschaft bereits beendet ist und die vor oder während ihrer Schwangerschaft geritten sind. 


\section{Fragestellungen}

Ziel der vorliegenden Arbeit ist die Beantwortung folgender Fragen:

1. Ist das Studienkollektiv mit einem Normalkollektiv von Schwangeren (Niedersächsische Perinatalstudie) vergleichbar (Strukturvergleich)?

2. Haben Reiterinnen ein erhöhtes Abortrisiko?

3. Haben Reiterinnen ein erhöhtes Risiko für Frühgeburten?

4. In welchen Parametern unterscheiden sich Schwangerschaften mit einem Entbindungstermin (ET) bis Woche $36+0$ (Frühgeburten) und Schwangerschaften mit einem $\mathrm{ET}$ ab 36+1?

Ist ein erhöhtes Risiko für Frühgeburtlichkeit zu erkennen in Abhängigkeit von

- Intensität und Dauer des Reitens pro Woche (während der SWS),

- reiterlichen Disziplin (Dressur und Springreiten versus Freizeitreiten),

- zeitlicher Fortdauer des Reitens während der Schwangerschaft ,

- reiterlicher Erfahrung,

- reiterlicher Qualifikation?

5. Ist ein erhöhtes Risiko für protrahierte Geburtsverläufe oder Geburtsstillstände gemessen an der Häufigkeit vaginal-operativer Entbindungen und sekundärer Sectiones caesareae - erkennbar in Abhängigkeit von

- Intensität und Dauer des RWS pro Woche,

- reiterlicher Disziplin (Dressur, Springreiten, Freizeitreiten),

- zeitlicher Fortdauer des Reitens während der Schwangerschaft (Reiten bis 1., 2., 3. Trimenon),

- reiterlicher Erfahrung und Qualifikation?

6. Führt Reiten während der Schwangerschaft zu einem erhöhten Risiko für Entbindungen aus Beckenendlage?

Ist ein solches Risiko abhängig von

- Intensität und Dauer des Reitens pro Woche,

- reiterlicher Disziplin, 
- zeitlicher Fortdauer des Reitens während der Schwangerschaft?

7. Sind Reiterinnen während der Schwangerschaft durch das Unfallrisiko beim Reiten und beim Umgang mit dem Pferd gefährdet?

Ist das Unfallrisiko abhängig von

- Intensität und Dauer des Reitens pro Woche,

- reiterlicher Disziplin,

- reiterlicher Erfahrung und Qualifikation,

- weiteren Faktoren? 


\section{Material und Methoden}

Zur Beantwortung der in Kapitel 1 aufgeführten Fragen wurde eine retrospektive Befragung von Reiterinnen mit abgeschlossener Schwangerschaft durchgeführt. Hierfür wurde durch die Untersucherin ein Fragebogen mit 54 Fragen konzipiert. Die gynäkologischgeburtshilfliche Komponente wurde in Anlehnung an die Niedersächsische Perinatalstudie und an den Fragebogen des Baby-Care-Projekts (www.baby-care.de) konzipiert. Der reiterlich-sportmedizinische Teil wurde in Kooperation mit der Deutschen Reiterlichen Vereinigung und dem Institut für Angewandte Sportwissenschaften (www.ias-service.de) erarbeitet. Um das Projekt interessierten Teilnehmerinnen, Frauenärzten, Journalisten, Reiterverbänden und Reitlehrern zu präsentieren, wurde eine Homepage www.schwangerreiten.de aufgebaut und mit ausführlichen Informationen und Presseunterlagen zur Befragung ausgestattet. Außerdem wurden der Online-Fragebogen und Gästebuch auf diese Homepage gestellt. Die Antworten aus der Befragung wurden anonym direkt in eine Datenbankanwendung eingespeist, aus der anschließend der Datenexport nach Excel ohne Informationsverlust erfolgte.

Die Befragung wurde als einmalige Online-Befragung ohne Follow-up konzipiert. Auf die Befragung wurde im Dezember 2004 durch eine Pressemeldung der Deutschen Reiterlichen Vereinigung aufmerksam gemacht. Dieser Pressemeldung folgten weitere Presse-Aktivitäten der Autorin, Publikationen in Reiterzeitschriften, in Internet-Foren und in mehreren Tageszeitungen und Publikumszeitschriften. Zusätzlich verbreitete die Bundesvereinigung der Berufsreiter (BBR) die Umfrage unter ihren Mitgliedern. Die Befragung war im Untersuchungszeitraum bei aktiven Reiterinnen und Reitern durch diese Maßnahmen hinreichend bekannt.

Frauenärztinnen und Frauenärzte wurden durch die Zeitschrift „Frauenarzt“ informiert, die vom Berufsverband der Frauenärzte (BVF) und der Deutschen Gesellschaft für Gynäkologie und Geburtshilfe (DGGG) gemeinsam herausgegeben wird.

An der Umfrage teilnehmen konnte jede Frau, die vor oder während ihrer Schwangerschaft geritten ist, deren Schwangerschaft zum Zeitpunkt der Teilnahme an der Befragung durch Abort, Fehlgeburt oder Geburt beendet war, und deren Entbindung im Befragungszeitraum zwischen 1995 und 2005 lag. Für jede Schwangerschaft musste ein eigener, vollständiger Fragebogen ausgefüllt werden. Bei Teilnehmerinnen unter 18 Jahren (Alter bei Teilnahme an der Befragung) war die Teilnahme nur mit schriftlicher Einwilligung der Eltern möglich. Dieser Fall trat aber nicht auf. 
Die Teilnahme war anonym. In der Annahme, mit einem zusätzlichen Anreiz die Teilnehmerinnenzahlen zu erhöhen, wurden unter den Teilnehmerinnen zwei IntensivReitseminare an führenden Reitzentren und 50 Paar Reithandschuhe ausgelost. Die Angabe der persönlichen Daten war allerdings für die Teilnahme an der Untersuchung nicht obligatorisch. Die Daten für die Teilnahme an der Verlosung wurden getrennt von den Studiendaten erfasst.

Das positive Votum der Ethikkommission der Universität München wurde vor dem Start der Befragung eingeholt.

Frauen, die keinen Internetzugang hatten und an der Befragung teilnehmen wollten, erhielten den Fragebogen per Post. Die zurückgesandten Fragebögen wurden in die OnlineDatenbank eingetragen.

Die Fragebögen wurden von den Teilnehmerinnen selbständig ausgefüllt. Dies setzte Zeitschriftenlektüre bzw. aktive Internet-Nutzung voraus, Erfahrung mit Multiple-ChoiceBefragungen, eine ausreichende Kenntnis der deutschen Schriftsprache und ca. 20 Minuten konzentriertes Arbeiten. Der hohe Anteil von Teilnehmerinnen mit Hochschulreife (63\%) kann als Zeichen dafür interpretiert werden, dass diese Kriterien zu einer Teilnehmerselektion geführt haben. Ein möglicher Einfluss auf die Frühgeburtenrate und die Aussagefähigkeit der Daten wird in Kapitel 5.1 (S. 50 ff.) diskutiert.

In den Fragebögen wurden folgende Daten evaluiert (siehe Anhang)

- Allgemeine Daten,

- Alter bei Beginn der Schwangerschaft,

- BMI,

- Schulbildung,

- gynäkologische und geburtshilfliche Anamnese,

- sportliche Aktiväten vor der Schwangerschaft,

- reiterliche Qualifikation,

- reiterliche Aktivitäten (Disziplinen, Intensität, wöchentliche Reitdauer) vor der Schwangerschaft,

- Information des Frauenarztes über die reiterliche Aktivität,

- Dauer des Reitens während der Schwangerschaft,

- sportliche Aktiväten während der Schwangerschaft, 
- reiterliche Aktivitäten (Disziplinen, Intensität, wöchentliche Reitdauer) während der Schwangerschaft,

- Komplikationen und Erkrankungen während der Schwangerschaft,

- Stress- und Belastungsfaktoren,

- Nikotin-, Alkohol- und Koffein-Konsum,

- Kinderwunschbehandlung,

- Verlauf der Geburt,

- Outcome von Mutter und Kind.

Nach Dammrissen und Dammschnitten als möglichen Indizien für eine potentiell rigide, den Geburtsverlauf beeinträchtigende Beckenbodenmuskulatur wurde nicht gefragt, weil diese in erheblichem Umfang von der klinischen Tradition und dem Können von Hebamme und Geburtshelfer abhängig sind, und weil von den Teilnehmerinnen retrospektiv eine zuverlässige Angabe über Ausmaß und Lokalisation des Dammrisses bzw. Dammschnittes nicht zu erwarten war.

Die Online-Befragung startete im April 2005. Ziel war ein Rücklauf von mindestens 1000 vollständig ausgefüllten Fragebögen/Datensätzen. Am 15. September 2005 wurde die Untersuchung mit einem Bestand von 1881 Datensätzen geschlossen. 33 Teilnehmerinnen hatten ihre Fragebögen per Fax zurückgesandt, 143 per Post, 1710 hatten den Fragebogen im Internet ausgefüllt.

Die Rückmeldungen wurden zu etwa $80 \%$ durch die Publikationen in Reiterzeitschriften und -foren ausgelöst. Teilnehmerinnen, die nach der Geburt ihrer Kinder aus dem Reitsport ausgeschieden sind, wurden wegen zu geringer Resonanz von Frauen- und Familienzeitschriften nicht in befriedigender Weise erreicht. Es muss als wahrscheinlich angesehen werden, dass Reiterinnen, die den Reitsport während der Schwangerschaft aufgegeben und danach nicht wieder aufgenommen haben, in der Befragung unterrepräsentiert sind. Ob dies zu einer Verzerrung der Ergebnisse geführt haben kann, muss zum gegenwärtigen Zeitpunkt offen bleiben. Nach Abschluss der hier vorgelegten Arbeit ist eine weiterführende Befragungswelle geplant, die hierzu möglicherweise Aufschluss geben kann.

Vor der Auswertung zu den Einflüssen des Reitens auf die Frühgeburtlichkeit und auf den Geburtsverlauf wurden folgende Fragebögen entfernt: 39 unvollständig, offensichtlich fehlerhaft oder unplausibel ausgefüllte Fragebögen, 
16 Aborte und Fehlgeburten,

214 Entbindungen vor 1995,

26 noch nicht beendete Schwangerschaften,

28 Mehrlingsgeburten (Mehrlingsschwangerschaften bergen ein so erhebliches Risiko für eine vorzeitige Entbindung, dass nicht auszuschließen war, dass sie die Ergebnisse der Datenanalyse in Bezug auf die Frühgeburtlichkeit unzulässig verfälscht hätten).

Damit blieben 1558 Datensätze in der Auswertung:

1122 Primipare,

436 Multiparae.

Für Fragestellungen bezüglich des Unfallrisikos wurden die Datensätze der Primi- und Multiparae gemeinsam ausgewertet, für alle andere Fragestellungen getrennt.

In der Erhebung wurden neben den reiterlichen Daten und Angaben zur Schwangerschaft und Geburt auch Daten zu nicht-reiterlichen sportlichen Aktivitäten der Frauen vor und während der Schwangerschaft erhoben, ebenso zu Kinderwunschbehandlung, zu Krankheiten und Komplikationen während der Schwangerschaft. Diese haben nach statistischer Auswertung der Daten keinen Einfluss auf die zu untersuchende Fragestellung (Anhang Tab. A1). Sie werden deshalb an dieser Stelle nicht erörtert.

Zur Auswertung wurde mit Microsoft Excel 2003 gearbeitet. Signifikanzen wurden mit dem TTest ermittelt und nur bei einem $p<0.05$ berücksichtigt (T-Test $\geq 1,96$ ). Schwach signifikante Ergebnisse ( $T$-Test $<1,94$ und $\geq 1,64$ ) werden im Weiteren nur dort erwähnt, wo sie im Rahmen eindeutig signifikanter Zusammenhänge zur Abrundung eines Gesamtbildes beitragen können.

Qualitative Merkmale wurden nicht verglichen.

Bei der vergleichenden Strukturprüfung (eigenes Datenmaterial vs. Niedersächsische Perinatalstudie) wurden für die Daten der Reiterinnenstudie 95\%-Konfidenzintervalle berechnet. Die Durchführung eines T-Tests zur Prüfung der Signifikanz der Wertunterschiede war hier nicht möglich, da es sich bei den Perinataldaten nicht um eine Stichprobe, sondern um Daten einer Grundgesamtheit handelte. 
Auf Angaben der Odds-Ratios wurde bewusst verzichtet, weil es nicht möglich ist, Angaben zur Repräsentativität der Studiengruppe zu machen, und weil die Möglichkeit nicht ausgeschlossen werden kann, dass mit der Angabe von Odds-Ratios Risikobewertungen in die Diskussion einfließen, die der unsicheren Datenlage nicht angemessen sind.

Die Intervalle im folgenden Kapitel (4. Ergebnisse) werden deshalb wie folgt berechnet:

Abb. 1. Berechnung der Konfidenzintervalle.

\begin{tabular}{|l|l|}
\hline Konfidenzintervall bei Anteilswerten & Konfidenzintervall bei Mittelwerten \\
\hline$z \alpha=p \pm t \sqrt{\frac{p q}{n}}$ & $\mathrm{z} \alpha=\mathrm{M}+-\mathrm{t}(\mathrm{s} / \sqrt{ } \mathrm{n})$ \\
Wobei: & Wobei: \\
$\mathrm{p}=$ Anteilswert & $\mathrm{M}=$ Mittelwert der Stichprobe \\
$\mathrm{t}=$ Irrtumswahrscheinlichkeit & $\mathrm{t}=$ Irrtumswahrscheinlichkeit \\
$5 \%(1-\alpha)=0,950 \mathrm{t}=1,64 /(1-\alpha / 2)=1,96$ & $5 \%(1-\alpha)=0,950 \mathrm{t}=1,64 /(1-\alpha / 2)=1,96$ \\
$\mathrm{q}=1-\mathrm{p}$ & $\mathrm{s}=$ Standardabweichung \\
\hline
\end{tabular}

Signifikante Unterschiede liegen vor, wenn sich die Konfidenzintervalle nicht überschneiden. Es wird die übliche Irrtumswahrscheinlichkeit von 5\% unterlegt. Die Konfidenzintervallprüfungen werden überall dort angewendet, wo die Niedersächsischen Perinatalstudie als Grundgesamtheit herangezogen wird.

Abb. 2. Berechnung T-Test.

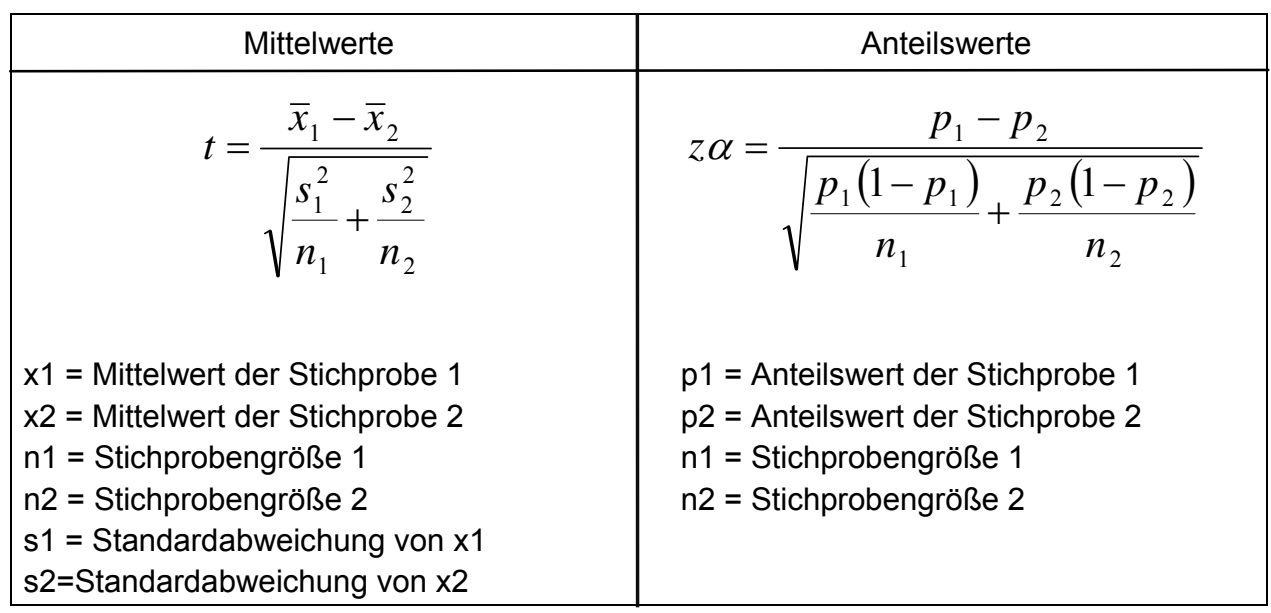

Signifikante Unterschiede liegen vor, wenn $t, z \alpha>1,96$ ist. Weitere Voraussetzungen sind, dass die Stichproben $n \geq 30$ und die Zellenbesetzungen $n>5$ sind. Es wird die übliche 
Irrtumswahrscheinlichkeit von 5\% unterlegt. Der T-Test wird verwendet, um Aussagen über unterschiedliche Gruppen der Studienteilnehmerinnen zu prüfen.

In den Tabellen sind signifikante Unterschiede mit s gekennzeichnet, nicht signifikante Unterschiede mit ns. 


\section{Ergebnisse}

\subsection{Vergleich mit der Niedersächsischen Perinatalstudie (Normalkollektiv)}

In der Befragung wurden insgesamt $\mathrm{n}=1881$ Interviews realisiert. Aufgrund der Art der Datenerhebung als Internetbefragung kann eine Strukturprüfung der realisierten Nettostichprobe gegenüber der Bruttostichprobe für ausgewählte Variablen (z.B. Alter, Schulbildung) nicht erfolgen.

Um die Struktur der Stichprobe zu beschreiben, wird diese für ausgewählte Variablen mit den Perinataldaten in Niedersachsen aus dem Jahr 2003 verglichen. Das Jahr 2003 wurde gewählt, weil die Datenerhebung der Reiterinnenstudie sich über die Jahre 1995 bis 2005 erstreckte.

Von den 1881 Rückläufen waren $n=39$ Datensätze unvollständig und $n=16$ Aborte, Fehloder Totgeburten vor der 25. Schwangerschaftswoche (SSW). Das Abortrisiko wird in Kapitel 4.3. erörtert (S. 27 f.). Da Fehl- und Totgeburten vor der 25. SSW in den Perinataldaten nicht erhoben werden, wurden diese Datensätze vor dem Vergleich mit der Niedersächsischen Perinatalstudie aus der Erhebung entfernt. N=214 Datensätze betrafen Geburten vor 1995, teilweise zurück bis 1965. Auch diese Datensätze wurden entfernt, ebenso 26 Datensätze mit Schwangerschaften, die zum Erhebungszeitraum noch nicht abgeschlossen waren.

Folgende Basisvariablen, die die Frühgeburtenrate stark determinieren und in den Perinataldaten reliabel vorliegen, werden in den Strukturvergleich einbezogen:

1. Alter nach vier Altersgruppen

2. Parität: Primiparae / Multiparae

3. Einlingsschwangerschaften

4. Entbindungsmodus

Die Perinataldaten wurden von der Ärztekammer Niedersachsen zur Verfügung gestellt. Lediglich die Basisdaten zum Entbindungsmodus wurden aus der Internetdokumentation www.zq-aekn.de (Auswertung 2003) entnommen.

Da ein Zusammenhang zwischen der schulischen Abschlussqualifikation und dem Frühgeburtsrisiko bekannt ist, wurde als weitere Prüfvariable die Schulbildung einbezogen. 
Diese Variable ist allerdings nicht in den Perinataldaten enthalten, so dass hier ein anderes Vorgehen gewählt werden musste (s.u.).

Bei der vergleichenden Strukturprüfung wurden für die Daten der Reiterinnenstudie 95\% Konfidenzintervalle berechnet. Die Durchführung z.B. eines T-Tests zur Prüfung der Signifikanz der Werteunterschiede war nicht möglich, da es sich bei den Perinataldaten nicht um eine Stichprobe, sondern um Daten einer Grundgesamtheit handelt.

Die Intervalle wurden nach den Formeln in Abbildung 1 errechnet (siehe S. 19).

Die Vergleichsanalyse zeigt, dass sich die Reiterinnenstichprobe hinsichtlich des Alters und der Parität signifikant von der Struktur der Perinataldaten unterscheidet. So haben sich jüngere Reiterinnen (bis zu 24 Jahren) wie auch ältere (35 Jahre und mehr) unterproportional an der Studie beteiligt.

Noch gravierender sind die Strukturunterschiede in der Parität. Während in den Perinataldaten 46\% Erstgebärende sind, sind es in der Reiterinnenstudie 72,1\%.

Der Grund für diese Verzerrung liegt möglicherweise darin begründet, dass Mehrfachgebärende in der Reiterinnenstudie für jedes geborene Kind einen Fragebogen ausfüllen sollten, was die Teilnahmeakzeptanz dieser Gruppe reduziert haben könnte. Zudem machen viele Reiterinnen, die kein eigenes Pferd und keine professionellen Ambitionen und Verpflichtungen haben, nach der Geburt des ersten Kindes eine Reitpause und wurden deshalb durch die Öffentlichkeitsarbeit in der reiterlichen Fachliteratur nicht erreicht. Wie bereits in Kapitel 3 erwähnt, war die redaktionelle Kooperationsbereitschaft in der Publikumspresse unbefriedigend. 
Tab. 1. Vergleichsanalyse Reiterinnenstudie und Niedersächsische Perinatalstudie.

\begin{tabular}{|c|c|c|c|c|c|c|c|c|}
\hline & & & & & \multicolumn{4}{|c|}{ Konfidenzintervall } \\
\hline & \multicolumn{2}{|c|}{ Reiterinnenstudie } & \multicolumn{2}{|c|}{ Perinataldaten } & \multirow{2}{*}{$\begin{array}{l}\text { Untere } \\
\text { Grenze }\end{array}$} & \multirow[t]{2}{*}{ Mittelwert } & \multirow{2}{*}{$\begin{array}{l}\text { Obere } \\
\text { Grenze }\end{array}$} & \multirow[t]{2}{*}{ Signifikanz } \\
\hline $\begin{array}{l}\text { Alter zu } \\
\text { Beginn der } \\
\text { SWS }\end{array}$ & abs. & $\%$ & abs. & $\%$ & & & & \\
\hline bis 24 Jahre & 191 & $12,3 \%$ & 12538 & $19,1 \%$ & $7,6 \%$ & $12,3 \%$ & $17,0 \%$ & $\mathrm{Ja}$ \\
\hline 25-29 Jahre & 546 & $35,0 \%$ & 17639 & $26,9 \%$ & $31,7 \%$ & $35,0 \%$ & $38,3 \%$ & $\mathrm{Ja}$ \\
\hline 30-34 Jahre & 594 & $38,1 \%$ & 21539 & $32,8 \%$ & $32,84 \%$ & $38,1 \%$ & $43,4 \%$ & $\mathrm{Ja}$ \\
\hline $35+$ & 287 & $14,5 \%$ & 13867 & $21,1 \%$ & $6,9 \%$ & $14,5 \%$ & $22,1 \%$ & Nein \\
\hline Summe & 1558 & $100 \%$ & 65583 & $100 \%$ & & & & \\
\hline
\end{tabular}

\begin{tabular}{|l|l|l|l|l|l|l|l|l|}
\hline \multicolumn{1}{|l|}{ Parität } & \multicolumn{1}{|l|}{ Ja } \\
\hline Primiparae & 1122 & $72,0 \%$ & 29957 & $45,7 \%$ & $69,8 \%$ & $72,0 \%$ & $74,2 \%$ & $\mathrm{Ja}$ \\
\hline Multiparae & 436 & $28,0 \%$ & 35626 & $54,3 \%$ & $24,5 \%$ & $28,0 \%$ & $31,5 \%$ & $\mathrm{Ja}$ \\
\hline Summe & 1558 & $100 \%$ & 65583 & $100 \%$ & & & \\
\hline
\end{tabular}

\begin{tabular}{|l|l|l|l|l|l|l|l|l|}
\hline \multirow{2}{*}{$\begin{array}{l}\text { Einlingl } \\
\text { Mehrling }\end{array}$} & \multicolumn{1}{|c|}{} \\
\hline Einling & 1530 & $98,2 \%$ & 64498 & $98,3 \%$ & $97,6 \%$ & $98,2 \%$ & $98,8 \%$ & Nein \\
\hline $\begin{array}{l}\text { Mehrlings- } \\
\text { geburt }\end{array}$ & 28 & $1,8 \%$ & 1085 & $1,7 \%$ & $0 \%$ & $1,8 \%$ & $5,9 \%$ & Nein \\
\hline Summe & 1558 & $100 \%$ & 65583 & $100 \%$ & & & \\
\hline
\end{tabular}

\begin{tabular}{|l|c|c|c|c|c|c|c|c|}
\hline $\begin{array}{l}\text { Entbindungs- } \\
\text { modus }\end{array}$ & \multicolumn{1}{|c|}{} \\
\hline Spontan/vag. & 1045 & $67,1 \%$ & 44541 & $68,0 \%$ & $64,7 \%$ & $67,1 \%$ & $69,5 \%$ & Nein \\
\hline $\begin{array}{l}\text { Vag-op. } \\
\text { Entbindung }\end{array}$ & 140 & $9,0 \%$ & 3871 & $5,9 \%$ & $5,0 \%$ & $9,0 \%$ & $13,0 \%$ & Nein \\
\hline $\begin{array}{l}\text { Sectio } \\
\text { primär und } \\
\text { sekundär }\end{array}$ & 373 & $23,9 \%$ & 16991 & $25,9 \%$ & $20,3 \%$ & $23,9 \%$ & $27,5 \%$ & Nein \\
\hline Summe & 1558 & $100 \%$ & 65583 & $100 \%$ & & & & \\
\hline
\end{tabular}

Keine signifikanten Unterschiede ergeben sich für die Variablen Einling/Mehrling sowie für den Entbindungsmodus. Allerdings ist der Anteil der vaginal-operativen Entbindungen wenn auch nicht signifikant - gegenüber den Daten aus der Perinatalstudie erhöht.

Zusammenfassend lässt sich feststellen, dass in der Reiterinnenstudie gegenüber der Niedersächsischen Perinatalstudie

- mehr Erstgebärende,

- weniger Schwangere unter 24 Jahre und

- weniger Schwangere über 35 Jahre vertreten sind. 
Diese Unterschiede lassen es sinnvoll erscheinen, die Ergebnisse aus der Reiterinnenstudie für die Fragestellung, ob Reiterinnen ein höheres Frühgeburtsrisiko haben als Nichtreiterinnen, zu gewichten.

\subsection{Ermittlung des Frühgeburtsrisikos bei Reiterinnen nach Gewichtung}

Um die Daten aus der vorliegenden Studie interpretieren zu können, wurden sie den Daten aus der Niedersächsischen Perinatalstudie (2003) gegenübergestellt. Aufgrund der Strukturunterschiede zwischen den Datensätzen ist ein Vergleich der Zielvariablen der Untersuchung (Geburtswoche) ohne eine Gewichtung der Daten der Reiterinnenstudie auf die Struktur der Perinataldaten nicht möglich.

\subsubsection{Gewichtung nach Alter}

Theoretisch kann eine altersbezogene Gewichtung für den Gesamtdatensatz der Reiterinnenstudie durchgeführt werden. Allerdings führt dies aufgrund der sehr geringen Fallzahlen bei Multiparae zu problematisch hohen Gewichtungsfaktoren von $>3$ in der unteren und $>2$ in der oberen Altersgruppe der Multiparae (Tab. 2).

Tab. 2. Gewichtungsfaktoren bei Gewichtung der Gesamtstichprobe (PP=Primiparae, M=Multiparae).

\begin{tabular}{|c|c|c|c|c|c|}
\hline Verteilung Perinataldaten & unter $\mathbf{2 5} \mathbf{J}$. & $\mathbf{2 5 - 2 9} \mathbf{J .}$ & $\mathbf{3 0 - 3 4} \mathbf{~ J .}$ & $\mathbf{3 5 +}$ & Summe \\
\hline PP & $13,2 \%$ & $13,6 \%$ & $13,0 \%$ & $5,9 \%$ & $100,0 \%$ \\
\hline MP & $5,9 \%$ & $13,3 \%$ & $19,8 \%$ & $15,3 \%$ & $100,0 \%$ \\
\hline Verteilung Reiterstudie & \multicolumn{5}{|l}{} \\
\hline PP & $12,6 \%$ & $28,6 \%$ & $25,0 \%$ & $6,4 \%$ & $100,0 \%$ \\
\hline MP & $1,5 \%$ & $7,5 \%$ & $11,7 \%$ & $6,7 \%$ & $100,0 \%$ \\
\hline Gewichtungsfaktoren & \multicolumn{5}{|l}{} \\
\hline PP & 1,05 & 0,48 & 0,52 & 0,92 & \\
\hline MP & 3,94 & 1,77 & 1,69 & 2,28 & \\
\hline
\end{tabular}

Aus diesem Grund werden die Daten der Reiterinnenstudie getrennt für Primi- und Multiparae gewichtet, was zur Folge hat, dass die Verteilungen der Zielvariablen der Untersuchung getrennt für Primi- und Multiparae untersucht werden müssen (Tab. 3).

Bei der Gewichtung nach Alter (Tab. 4) ergeben sich in der Reiterinnenstudie sowohl für Primi- als auch für Multiparae insgesamt signifikant geringere Frühgeburtenraten gegenüber den Perinataldaten 2003 in Niedersachsen. 
Tab. 3. Getrennte Gewichtung für Primiparae (PP) und für Multiparae(MP).

\begin{tabular}{|c|c|c|c|c|c|}
\hline Verteilung Perinataldaten & unter $\mathbf{2 5} \mathbf{J}$. & $\mathbf{2 5 - 2 9} \mathbf{J .}$ & $\mathbf{3 0 - 3 4} \mathbf{J .}$ & $\mathbf{3 5 +}$ & Summe \\
\hline PP & $29,0 \%$ & $29,8 \%$ & $28,5 \%$ & $12,8 \%$ & $100,0 \%$ \\
\hline MP & $10,8 \%$ & $24,5 \%$ & $36,5 \%$ & $28,2 \%$ & $100,0 \%$ \\
\hline Verteilung Reiterstudie & \multicolumn{5}{|l}{} \\
\hline PP & $17,4 \%$ & $39,4 \%$ & $34,5 \%$ & $8,8 \%$ & $100,0 \%$ \\
\hline MP & $5,4 \%$ & $27,4 \%$ & $42,7 \%$ & $24,4 \%$ & $100,0 \%$ \\
\hline Gewichtung Alter und Parität & \multicolumn{5}{|c|}{} \\
\hline PP & 1,66 & 0,76 & 0,83 & 1,46 & \\
\hline MP & 1,99 & 0,89 & 0,85 & 1,15 & \\
\hline
\end{tabular}

Tab. 4. Frühgeburtenraten gewichtet nach Alter.

\begin{tabular}{|c|c|c|c|c|c|}
\hline \multicolumn{6}{|l|}{ Primiparae } \\
\hline & Gesamt & unter $25 \mathrm{~J}$. & 25-29 J. & $30-34 \mathrm{~J}$. & $35+$ \\
\hline Frühgeburtenrate NDS 2003 & $10,0 \%$ & $9,4 \%$ & $9,2 \%$ & $10,4 \%$ & $11,88 \%$ \\
\hline$n=$ & 1122 & 170 & 433 & 407 & 112 \\
\hline Frühgeburten $n=$ & 70 & 11 & 28 & 23 & 8 \\
\hline Frühgeburtenrate Reiterstudie ungewichtet & $6,2 \%$ & $6,5 \%$ & $6,5 \%$ & $5,7 \%$ & $7,1 \%$ \\
\hline n gewichtet $=$ & 1113 & 282 & 329 & 338 & 164 \\
\hline $\begin{array}{l}\text { Frühgeburtenrate Reiterstudie } \\
\text { gewichtet für Alter }\end{array}$ & $7,9 \%$ & $9,2 \%$ & $7,8 \%$ & $6,9 \%$ & $7,8 \%$ \\
\hline \multicolumn{6}{|l|}{ Konfidenzintervall 95\% } \\
\hline Obere Grenze & $9,4 \%$ & $12,1 \%$ & $10,5 \%$ & $9,5 \%$ & $11,90 \%$ \\
\hline Mittelwert & $7,9 \%$ & $9,2 \%$ & $7,8 \%$ & $6,9 \%$ & $7,8 \%$ \\
\hline Untere Grenze & $6,4 \%$ & $6,3 \%$ & $5,1 \%$ & $4,3 \%$ & $3,7 \%$ \\
\hline Signifikant & $\mathrm{Ja}$ & Nein & Nein & $\mathrm{Ja}$ & Nein \\
\hline \multicolumn{6}{|l|}{ Multiparae } \\
\hline & Gesamt & unter $25 \mathrm{~J}$. & 25-29 J. & 30-34 J. & $35+$ \\
\hline Frühgeburtenrate NDS 2003 & $7,4 \%$ & $8,6 \%$ & $7,0 \%$ & $7,0 \%$ & $8,0 \%$ \\
\hline$n=$ & 436 & 23 & 107 & 196 & 110 \\
\hline Frühgeburtenrate Reiterstudie ungewichtet & $4,6 \%$ & $11,1 \%$ & $2,9 \%$ & $6,1 \%$ & $2,5 \%$ \\
\hline $\mathrm{n}=$ & 434 & 46 & 95 & 167 & 127 \\
\hline $\begin{array}{l}\text { Frühgeburtenrate Reiterstudie gewichtet } \\
\text { für Alter }\end{array}$ & $4,9 \%$ & $11,1 \%$ & $2,9 \%$ & $6,1 \%$ & $2,5 \%$ \\
\hline \multicolumn{6}{|l|}{ Konfidenzintervall 95\% } \\
\hline Obere Grenze & $6,0 \%$ & $19,5 \%$ & $5,9 \%$ & $9,6 \%$ & $5,1 \%$ \\
\hline Mittelwert & $4,9 \%$ & $11,1 \%$ & $2,9 \%$ & $6,1 \%$ & $2,5 \%$ \\
\hline Untere Grenze & $3,0 \%$ & $2,7 \%$ & $0,0 \%$ & $2,6 \%$ & $0,0 \%$ \\
\hline Signifikant & $\mathrm{Ja}$ & Nein & $\mathrm{Ja}$ & Nein & $\mathrm{Ja}$ \\
\hline
\end{tabular}




\subsubsection{Gewichtung nach Schulbildung}

Die Stichprobe der Reiterinnen enthält im Vergleich zum Bildungsstand bei Reitern insgesamt, der dem Bildungsniveau der Gesamtbevölkerung in Deutschland entspricht (Deutsche Reiterliche Vereinigung, 2001), einen überproportionalen Anteil an Frauen mit Abitur und einen unterproportionalen Anteil an Frauen mit einfacher Schulbildung (siehe Kapitel 3, Material und Methoden). Auf der Grundlage einer kumulierten Auswertung von repräsentativen Befragungsdaten von Frauen im Alter von 18 bis 45 Jahren im Land Niedersachsen verfügen dort im Jahr 2003

- $31 \%$ über einen Hauptschulabschluss,

- $51 \%$ über einen Realschulabschluss und

- $18 \%$ über einen Gymnasialabschluss.

In der Reiterinnenstudie verfügten

- $6 \%$ über einen Hauptschulabschluss,

- $31 \%$ über einen Realschulabschluss,

- $63 \%$ über einen Gymnasialabschluss.

Deshalb wurden die Daten der Reiterinnenstudie zusätzlich nach Schulabschluss gewichtet. Die Ergebnisse sind in Tab. 5 und Tab. 6 zusammengefasst. Eine Gewichtung der Gesamtrate an Frühgeburtenrate getrennt für Primi- und Multiparae nach Schulbildung ergibt für Primiparae keine signifikanten Unterschiede mehr. Bei bei Multiparae ist die Frühgeburtenrate auch nach Gewichtung hinsichtlich des Schulabschlusses weiterhin signifikant geringer als in den Perinataldaten.

Tab. 5. Gewichtung nach Schulbildung (Primiparae).

\begin{tabular}{|c|c|c|c|c|}
\hline Primiparae & Gesamt & Hauptschule & Realschule & Gymansium \\
\hline$n=$ & 1122 & 67 & 341 & 714 \\
\hline Verteilung IST & $100 \%$ & $6 \%$ & $31 \%$ & $63 \%$ \\
\hline Verteilung SOLL & $100 \%$ & $31,0 \%$ & $51,0 \%$ & $18,0 \%$ \\
\hline Zahl der Frühgeburten abs. & 86 & 5 & 33 & 48 \\
\hline Frühgeburtenrate ungewichtet & $7,7 \%$ & $7,9 \%$ & $9,7 \%$ & $6,7 \%$ \\
\hline Weight Bildung & & 5,19 & 1,68 & 0,28 \\
\hline Fallzahl gewichtet & 1122 & 348 & 572 & 202 \\
\hline Erwartungswerte Frühgeburten & 97 & 27 & 56 & 14 \\
\hline $\begin{array}{l}\text { Frühgeburtenrate gesamt } \\
\text { gewichtet nach Bildung }\end{array}$ & $8,6 \%$ & & & \\
\hline \multicolumn{2}{|l|}{ Konfidenzintervall 95\% } & & & \\
\hline Obere Grenze & $10,8 \%$ & & & \\
\hline Mittelwert & $8,6 \%$ & & & \\
\hline Untere Grenze & $6,4 \%$ & & & \\
\hline Signifikant & Nein & & & \\
\hline
\end{tabular}


Tab. 6. Gewichtung nach Schulbildung (Multiparae)

\begin{tabular}{|c|c|c|c|c|}
\hline Multiparae & Gesamt & Hauptschule & Realschule & Gymansium \\
\hline$n=$ & 436 & 28 & 136 & 278 \\
\hline Verteilung IST & $101 \%$ & $6,4 \%$ & $31,2 \%$ & $63,8 \%$ \\
\hline Verteilung SOLL & $100 \%$ & $31,0 \%$ & $51,0 \%$ & $18,0 \%$ \\
\hline Zahl der Frühgeburten & 14 & 1 & 5 & 8 \\
\hline Frühgeburtenrate ungewichtet & $3,2 \%$ & $3,6 \%$ & $3,7 \%$ & $2,9 \%$ \\
\hline Weight Bildung & & 4,83 & 1,64 & 0,28 \\
\hline Fallzahl gewichtet & 436 & 135 & 222 & 78 \\
\hline Erwartungswerte Frühgeburten & 15 & 5 & 8 & 2 \\
\hline $\begin{array}{l}\text { Frühgeburtenrate gesamt } \\
\text { gewichtet nach Bildung }\end{array}$ & $3,5 \%$ & & & \\
\hline \multicolumn{2}{|l|}{ Konfidenzintervall 95\% } & & & \\
\hline Obere Grenze & $5,8 \%$ & & & \\
\hline Mittelwert & $3,5 \%$ & & & \\
\hline Untere Grenze & $1,2 \%$ & & & \\
\hline Signifikant & $\mathrm{Ja}$ & & & \\
\hline
\end{tabular}

Zusammenfassend und vor der Prüfung weiterer Einflussfaktoren ist damit festzustellen, dass die gewichtete Reiterinnenstichprobe gegenüber den Perinataldaten in Niedersachsen in der Gruppe der Primiparae keine erhöhten Frühgeburtsraten aufweist, in der Gruppe der Multiparae eine signifikant niedrigere Frühgeburtenrate.

\subsection{Abortrisiko}

Genaue Zahlen zur Aborthäufigkeit existieren nicht. Es wird angenommen, dass ab Konzeption zwischen $10 \%$ und 15\% aller Schwangerschaften in einem Abort enden (Baltzer, 2006). In einer Auswertung der dänischen Kohortenstudie wird eine Abortrate von 3,6\% ab Bekanntwerden der Schwangerschaft berichtet (Madson 2007). Um die Frage nach der Aborthäufigkeit bei Reiterinnen zu evaluieren, waren im Studienaufruf alle Frauen zur Teilnahme aufgerufen worden, die vor oder während ihrer Schwangerschaft geritten sind, und bei denen die Schwangerschaft beendet war - gleichgültig, ob es sich um einen Abort oder um eine Geburt eines lebensfähigen Kindes gehandelt hatte.

In dem gesamten Datenpool der vollständig abgegebenen Datensätze $(n=1816)$ fanden sich 16 Teilnehmerinnen $(0,9 \%)$, bei denen die Schwangerschaft mit einem Abort vor Erreichen der Lebensfähigkeit des Kindes (beendete 24. Schwangerschaftswoche) geendet hat. In einem Fall handelte es sich um die Folge eines Reitunfalles, in zwei weiteren um eine 
Mehrlingsschwangerschaft. Drei Teilnehmerinnen gaben Fehlbildungen in der Familie an. Alle Daten können der Tabelle A3 im Tabellenband entnommen werden.

Die Gruppe der Frauen mit Abort unterschied sich in den folgenden Punkten nicht von den Teilnehmerinnen, deren Schwangerschaft zur Entbindung eines lebensfähigen Kindes geführt hat:

- Alter,

- reiterliche Aktivitäten vor und während der Schwangerschaft,

- geburtshilfliche und gynäkologische Anamnese,

- Kaffee-, Nikotin- und Alkoholkonsum während der Schwangerschaft.

Deutlich sichtbar, aber wegen der kleinen Gruppengröße nicht signifikant war der Unterschied im BMI: In der Gruppe der Reiterinnen mit Abort/Fehlgeburt war ein BMI von $<20$ mit 37,5\% häufiger als in der Gruppe mit Entbindungen nach Erreichen der 25. Woche $(20,3 \%)$. Ein BMI von $>25$ kam in der Abort-/Fehlgeburtengruppe nicht vor, dagegen in $15,1 \%$ bei den Reiterinnen mit Entbindung nach der 25. Woche.

Unterschiede, die trotz der sehr geringen Gruppengröße signifikant $(p<0,05)$ waren, fanden sich bei folgenden Parametern:

- Angabe „Der Frauenarzt hat mir vom Reiten während der Schwangerschaft abgeraten“ ( $37,5 \%$ vs. $12,3 \%)$,

- Fehlbildungen in der Familie der Mutter (18,8\% s. 3,5\%),

- Angabe „Bei meiner Arbeit musste ich mich regelmäßig stark konzentrieren“ (87,5\% vs. $69,7 \%)$,

- Angabe „Bei meiner Arbeit war ich häufig zu schnellen Entscheidungen gezwungen“ ( $81,3 \%$ vs. $56,4 \%)$, wobei diese Angaben keine objektiven Aussagen über die tatsächliche Belastung am Arbeitsplatz ermöglichen.

Teilnehmerinnen, deren Schwangerschaft in einem Abort oder einer Fehlgeburt endete, bezeichneten ihren sportlichen Trainingsstand vor und während der Schwangerschaft als besser und gaben häufiger an, neben dem Reiten noch weitere Sportarten auszuüben als Teilnehmerinnen, deren Schwangerschaft zur Entbindung eines lebensfähigen Kindes führte. Diese Unterschiede waren wegen der kleinen Gruppengröße nicht signifikant. Mögliche Korrelationen zwischen Sport und Aborthäufigkeit werden in Kapitel 5.4 ab S. 61 diskutiert.

Aufgrund der vorliegenden Daten kann die Annahme, dass das Reiten in der Schwangerschaft das Abortrisiko erhöhen würde, nicht bestätigt werden. 


\subsection{Einfluss unterschiedlicher Reitweisen auf die Frühgeburtlichkeit}

Für die Frage, ob reiterliches Training in der Schwangerschaft zu einem erhöhten Risiko für Frühgeburtlichkeit führt und ob dieses Risiko abhängig ist von der Art und Intensität des Trainings, wurden die Primiparae mit Einlings-Geburten $(n=1122)$ in zwei Gruppen aufgeteilt:

- Entbindungen ab Woche $37+0$ (Entbindungen am Termin, $n=1052$ ) und

- Entbindungen bis zur Woche 36+6 (Frühgeburten, $n=70$ ).

Die Gesamtübersicht ist in Tabelle A1 im Tabellenband enthalten.

Die Auswertung zeigt, dass es zwischen den beiden Gruppen keinerlei Unterschiede gibt hinsichtlich

- reiterlichen Disziplinen vor und während der Schwangerschaft (Abb. 3),

- reiterlicher Erfahrung, Qualifikation und Professionalität (Abb. 4),

- wöchentlicher Trainingszeit im Sattel (Abb. 5).

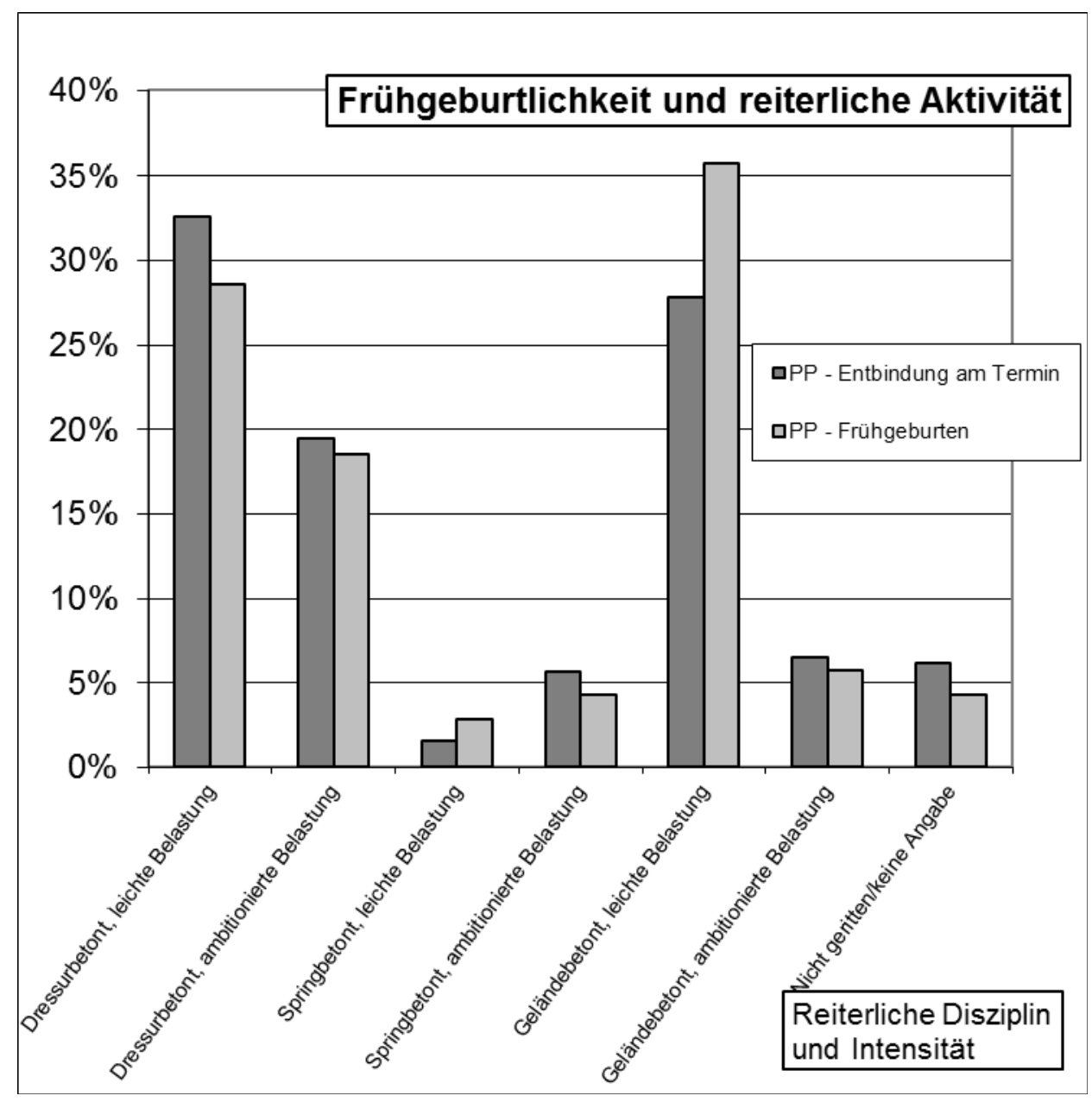

Abb. 3. Reiterliche Schwerpunkte und Aktivität bei Primiparae mit Entbindung am Termin und mit Frühgeburt. Die reiterlichen Aktivitäten sind in beiden Gruppen weitestgehend identisch. Unterschiede sind nicht signifikant. 


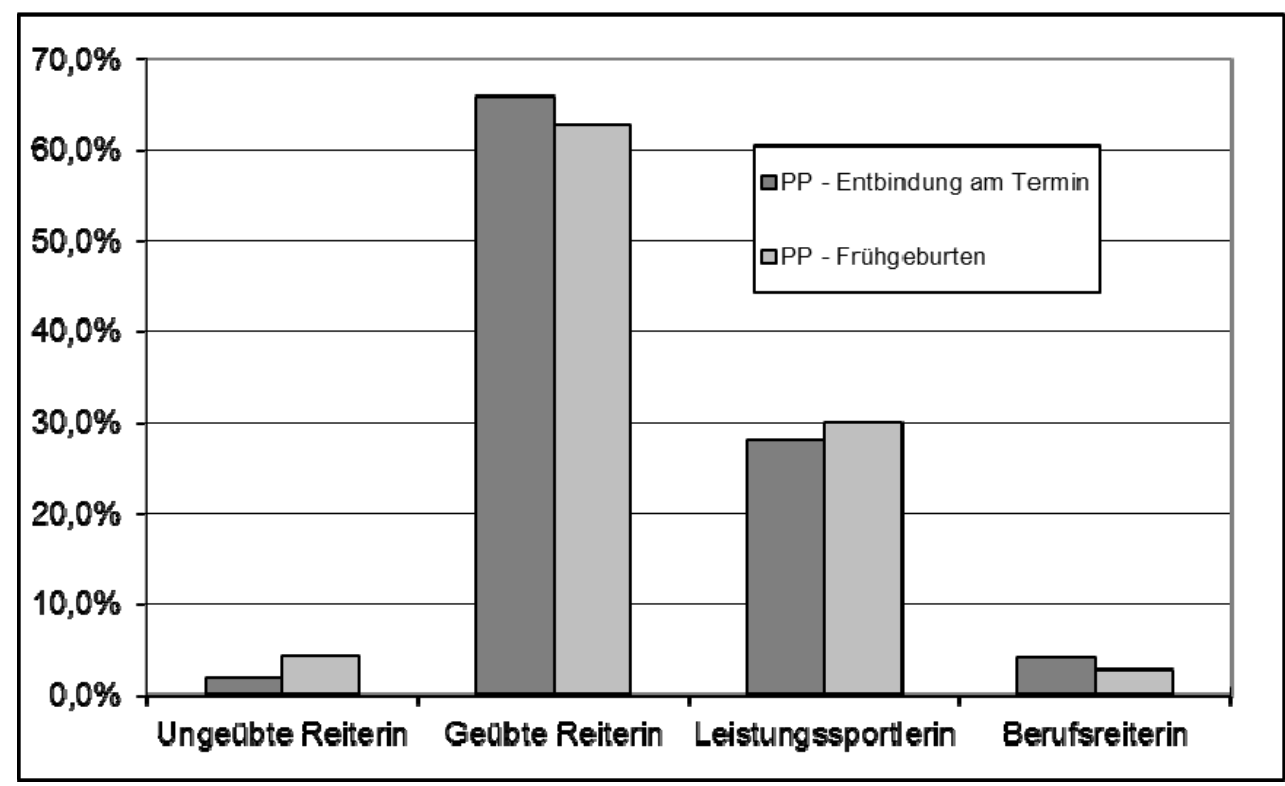

Abb. 4. Reiterliche

Erfahrung bei

Primiparae mit

Entbindung am

Termin und mit

Frühgeburt. Der

reiterliche

Erfahrungs- und

Trainingsstand ist

in beiden Gruppen

weitgehend

identisch.

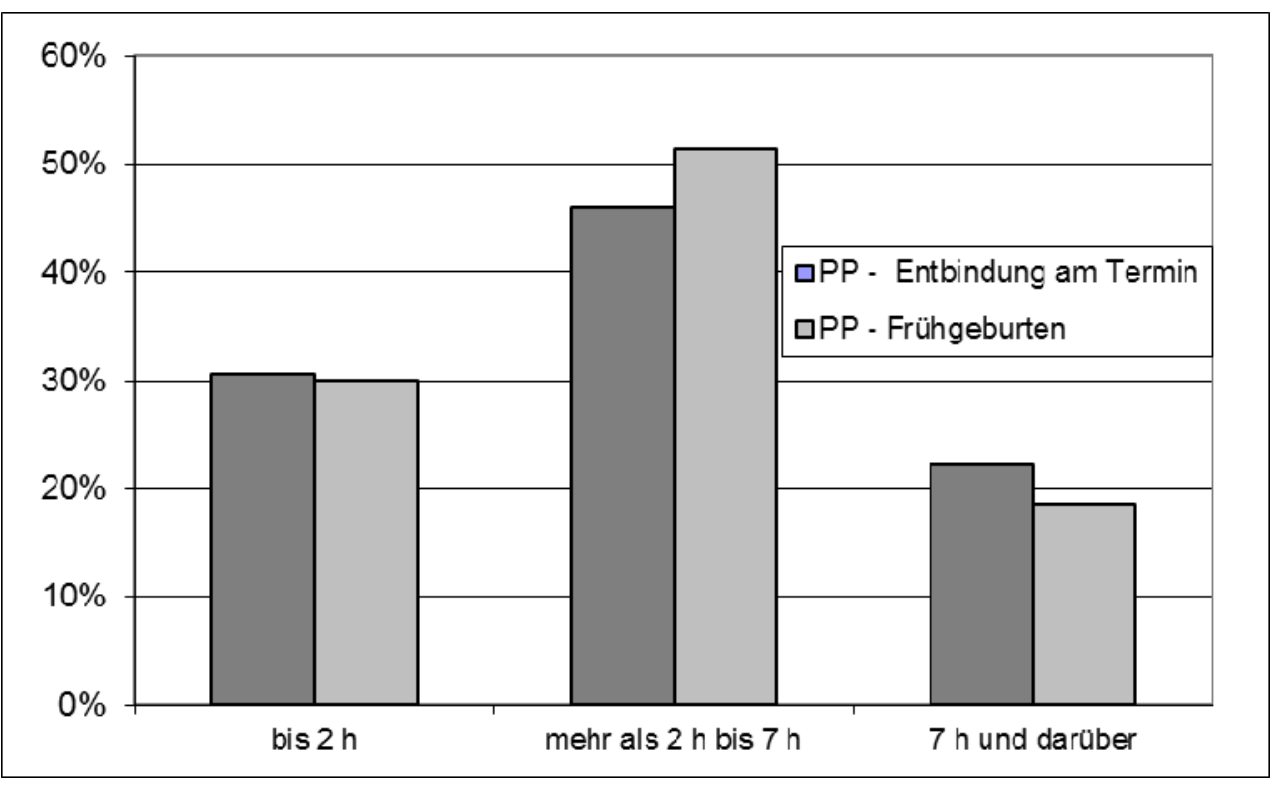

Abb. 5.

Wöchentliche

Trainingszeit im

Sattel während

der

Schwangerschaft bei Primiparae mit

Entbindung am

Termin und mit

Frühgeburt. Die

wöchentliche

Trainingszeit ist in

beiden Gruppen

weitgehend

identisch.

\subsubsection{Weiterreiten während der Schwangerschaft - inverse Korrelation zur Frühgeburtlichkeit}

Teilnehmerinnen, deren Schwangerschaft mit einer Frühgeburt endet, hören durchschnittlich einen Monat früher mit dem Reiten auf als Reiterinnen mit Entbindung am Termin (Schwangerschaftswoche 24,7 vs. 29,2 ); diese Differenz ist trotz der hohen Standardabweichungen signifikant (Abb. 6 und Tab. 7). 


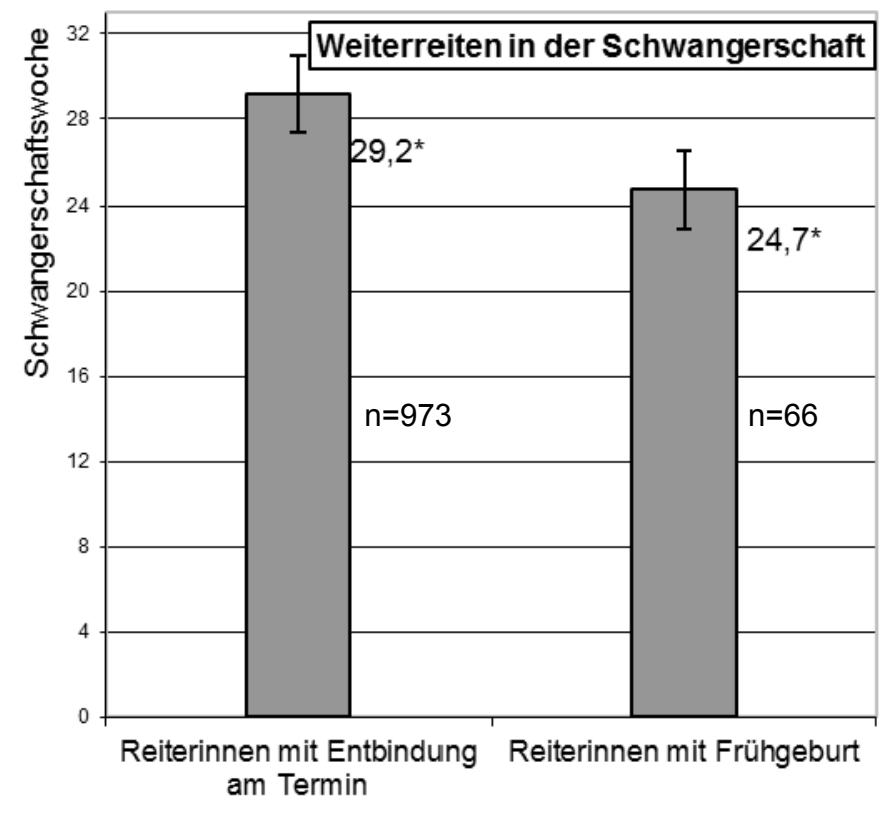

Abb. 6. Weiterreiten während der Schwangerschaft - Reiterinnen mit Entbindung am Termin vs.

Reiterinnen mit Frühgeburt.

Tab. 7. Weiterreiten während der Schwangerschaft. Reiterinnen mit Entbindung am Termin vs. Reiterinnen mit Frühgeburt (PP = Primiparae, ET = Entbindung am Termin, FG = Entbindung per Frühgeburt).

\begin{tabular}{|l|c|c|c|c|}
\hline $\begin{array}{l}\text { Nach Feststellung der Sws } \\
\text { weitergeritten (n=1039) }\end{array}$ & $\begin{array}{l}\text { Absolut PP } \\
\text { mit ET }\end{array}$ & Prozent & $\begin{array}{l}\text { Absolut PP } \\
\text { mit FG }\end{array}$ & Prozent \\
\hline Ja & 973 & $92,5 \%$ & 66 & $94,3 \%$ \\
\hline $\begin{array}{l}\text { Und zwar bis zur } \\
\text { Schwangerschaftswoche }\end{array}$ & $\mathbf{2 9 , 2}$ & & 24,7 & \\
\cline { 1 - 3 } St-Abw. & 9,0 & & 7,5 & \\
\cline { 1 - 4 } T-Test & \multicolumn{2}{|c|}{4,6} \\
\hline
\end{tabular}

Auch finden sich bei Schwangeren mit Frühgeburt weniger Reiterinnen, die während der Schwangerschaft täglich reiten $(7,1 \%$ vs. $16,1 \%$, Abb. 7 und Tab. 8$)$. 


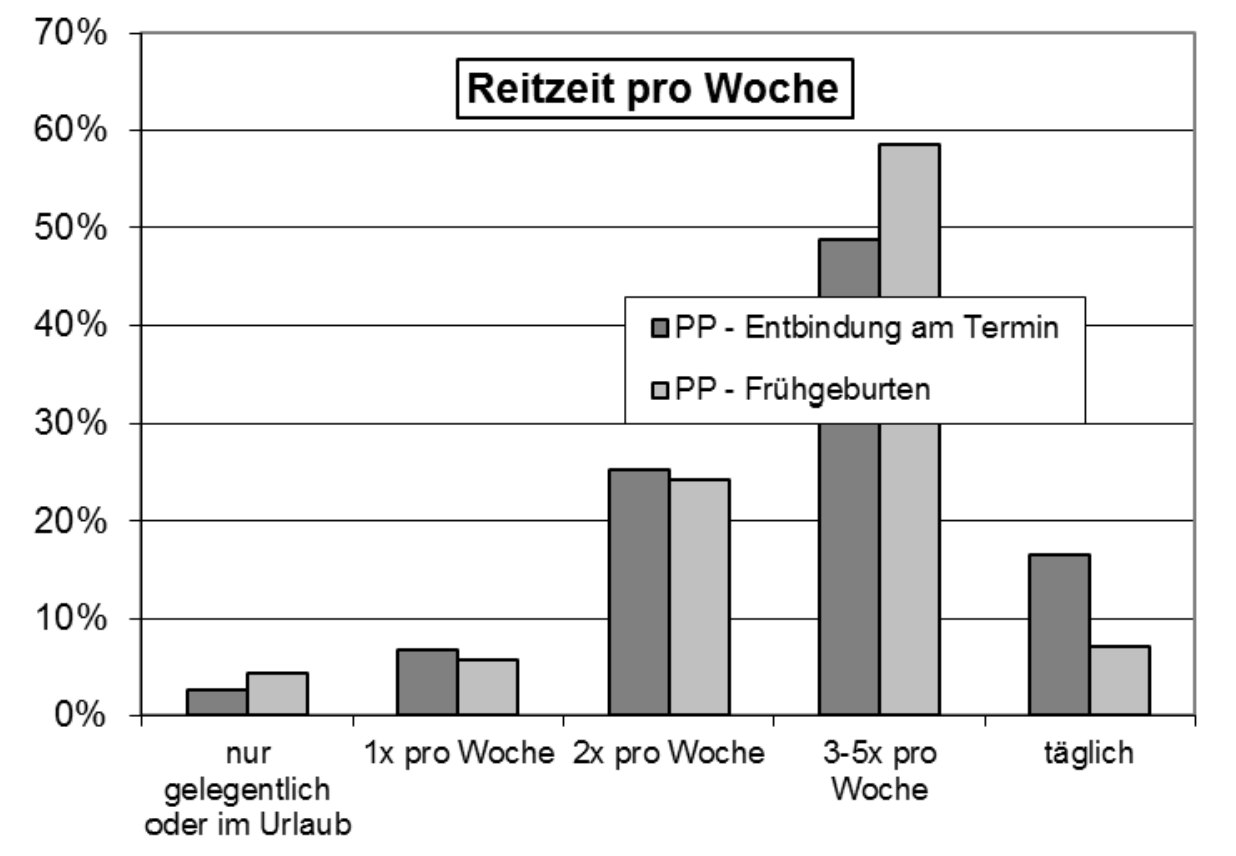

Abb. 7. Reitzeit pro

Woche -

Reiterinnen

(Primiparae) mit

Entbindung am

Termin vs.

Reiterinnen mit

Frühgeburt.

(Unterschiede nicht

signifikant, siehe

Tab. 8)

Tab. 8. Reittage pro Woche bis zur Beendigung des Reitens.

\begin{tabular}{|l|c|c|c|c|c|c|}
\hline $\begin{array}{l}\text { Reittage pro Woche bis } \\
\text { zur Beendigung des } \\
\text { Reitens (n=1122) }\end{array}$ & \multicolumn{2}{|l|}{$\begin{array}{l}\text { Reiterinnen (PP) mit } \\
\text { Entbindung am Termin }\end{array}$} & $\begin{array}{l}\text { Reiterinnen (PP) } \\
\text { mit Frühgeburt }\end{array}$ & $\begin{array}{l}\text { Signi- } \\
\text { fikanz }\end{array}$ & T-Test \\
\hline & $\mathrm{n}$ & $\%$ & $\mathrm{n}$ & $\%$ & \\
\hline & 1052 & $100 \%$ & 70 & & \\
\hline $\begin{array}{l}\text { nur gelegentlich oder im } \\
\text { Urlaub }\end{array}$ & 28 & $2,7 \%$ & 3 & $4,3 \%$ &.$/$. \\
\hline 1x pro Woche & 71 & $6,7 \%$ & 4 & $5,7 \%$ &.$/$. & \\
\hline 2x pro Woche & 265 & $25,2 \%$ & 17 & $24,3 \%$ & $\mathrm{~ns}$ & \\
\hline 3-5x pro Woche & 513 & $48,8 \%$ & 41 & $58,6 \%$ & $\mathrm{~ns}$ & 0,1 \\
\hline Täglich & 175 & $16,6 \%$ & 5 & $7,1 \%$ & $\mathrm{~ns}$ & 1,22 \\
\hline
\end{tabular}

Ein erhöhtes Risiko für Frühgeburtlichkeit in Abhängigkeit von der Dauer des täglichen oder wöchentlichen Reitens, des reiterlichen Niveaus oder der reiterlichen Disziplinen - Dressur, Springen, Gelände - kann in dieser Untersuchung mit den vorliegenden Daten von überwiegend gut trainierten und erfahrenen Reiterinnen nicht nachgewiesen werden.

Reiterinnen, deren Schwangerschaft vor Beendigung der 37. Schwangerschaftswoche endet, belasten sich reiterlich weniger stark als Schwangere, die am Entbindungstermin entbinden; allerdings sind die Unterschiede wegen der kleinen Gruppengröße (Tägliches Reiten $\mathrm{n}=5$ ) nicht signifikant. 


\subsection{Weitere Risiken für Frühgeburtlichkeit}

Es wurden weitere Parametern untersucht, in denen sich die Gruppe der Reiterinnen mit Frühgeburt (FG) und die Gruppe der Reiterinnen mit Entbindung am Termin (ET) unterscheiden könnten. Die Details sind Tab. A1 und A2 im Tabellenband zu entnehmen.

Keine Unterschiede in beiden Gruppen fanden sich hinsichtlich

- Alter,

- $\mathrm{BMI}$,

- gynäkologischer Anamnese,

- Schulabschluss,

- sportlichem Trainingszustand vor der Schwangerschaft,

- reiterlichem Leistungsstand,

- Reittagen pro Woche und täglicher Reitdauer vor der Schwangerschaft,

- reiterlichen Disziplinen und Leistungsorientierung vor der Schwangerschaft,

- Information und Empfehlung des Frauenarztes,

- Weiterreiten während der Schwangerschaft im ersten Trimenon,

- Schwangerschaftsbeschwerden im Zusammenhang mit dem Reiten (harter Bauch, Ziehen im Unterleib, Rückenschmerzen),

- reiterlichen Disziplinen und Leistungsorientierung während der Schwangerschaft,

- Änderungen des Reitstils mit Rücksicht auf die Schwangerschaft,

- Unfällen beim Reiten oder beim Umgang mit dem Pferd,

- sportlichem Trainingszustand während der Schwangerschaft,

- Schichtarbeit,

- körperlich schwerer Arbeit,

- Zwang zu starker Konzentration oder zu schnellen Entscheidungen, Arbeit mit Chemikalien.

In der FG-Gruppe finden sich als mögliche Ursachen der Frühgeburtlichkeit häufiger $(p<0,05)$

- Bluthochdruck während der Schwangerschaft (14,3\% vs. $4,5 \%)$,

- vorzeitige Wehentätigkeit nach Beendigung der Reitzeit (17,1\% vs. 7,2\%),

- vorzeitiger Blasensprung nach Beendigung der Reitzeit (24,3\% vs. 3,5\%),

- Zervixinsuffizienz nach Beendigung der Reitzeit (11,4\% vs. 2,9\%),

- Plazenta-Insuffizienz $(11,4 \%$ vs. $1,5 \%)$. 
Folgende Parameter treten bei Reiterinnen mit Frühgeburt mit schwacher Signifikanz $(p<0,1)$ gehäuft auf:

- Vorzeitige Wehentätigkeit während der Reitzeit (7,1\% vs. 3,1\%).

- vorzeitiger Blasensprung während der Reitzeit (5,7\% vs. $1,0 \%)$,

- Zervixinsuffizienz während der Reitzeit (5,7\% vs. $2,1 \%)$,

- Amnioninfektionssyndrom (2,9\% vs. $0,2 \%)$,

- Plazenta-Ablösung (2,9\% vs. $0,3 \%)$,

- Präeklampsie $(5,7 \%$ vs. $1,9 \%)$,

- Eklampsie/HELLP-Syndrom (2,9\% s. 0,7\%),

- Blutungen $(11,4 \%$ vs. $4,5 \%)$,

- Blutungen während der Reitzeit (10,0\% vs. 3,7\%).

Bei den nicht-medizinischen Parametern fällt folgender signifikanter Unterschied auf $(p<0,05)$ :

- In der FG-Gruppe geben signikant mehr Frauen an, dass sie sich durch Arbeit, Beruf, Familie oder anderes stark oder sehr stark belastet fühlen ( $39 \%$ vs. $21 \%)$, wobei das Auftreten der Belastungen selbst von den Frauen mit ET und mit FG als gleich häufig angegeben wird.

Schwach signifikant sind folgende Unterschiede in der Lebensführung:

- Koffeinkonsum wird in der FG-Gruppe häufiger angegeben (41,4\% vs. 36,6\%);

- Nikotinkonsum wird in der FG-Gruppe häufiger angegeben (14,3\% s. 8,9\%).

\section{Fazit}

Schwangerschaftskomplikationen und hohe Stressbelastung werden bei Reiterinnen mit Frühgeburten signifikant häufiger gefunden als bei Reiterinnen mit Entbindung am Termin.

Vorzeitige Wehen, Zervixinsuffizienz und vorzeitiger Blasensprung treten bei Reiterinnen mit Frühgeburt gelegentlich bereits während der aktiven Reitzeit auf. Die Unterschiede zu Reiterinnen mit Entbindung am Termin sind allerdings nicht signifikant. Aus den Daten kann deshalb nicht geschlossen werden, dass das Reiten selbst vorzeitige Wehen, Zervixinsuffizienz und vorzeitigen Blasensprung auslösen und damit das Risiko für eine Frühgeburtlichkeit erhöhen würde. 
Vorzeitige Wehen, Zervixinsuffizienz und vorzeitiger Blasensprung treten bei Reiterinnen mit Frühgeburt signifikant häufiger auf als bei Reiterinnen mit Entbindung am Termin, wobei diese Ereignisse meist erst nach Beendigung der reiterlichen Aktivität berichtet werden. Ein direkter Zusammenhang zwischen dem Sport und den Frühgeburtsbestrebungen kann nicht nachgewiesen werden.

\subsection{Reiten und vorzeitige Wehen}

Frauen, die in der Schwangerschaft weiterreiten, wünschen sich ein Regulativ, um selbst festzustellen und entscheiden zu können, wann sie den Sport beenden müssen. Vor allem für Berufsreiterinnen, die mehrere Stunden täglich im Sattel arbeiten, ist die Frage von Bedeutung, wann sie die Belastung reduzieren sollten.

Der Parameter „Vorzeitige Wehen“ mit seinen subklinischen Varianten dient traditionell als Gradmesser für die Stabilität einer Schwangerschaft. Deshalb wurde der Parameter „Vorzeitige Wehen“ (VW) auf seinen prognostischen Wert hinsichtlich der Frühgeburtlichkeit bei Reiterinnen überprüft. Nur von $n=549$ Primiparae lagen Angaben dazu vor, ob in ihrer Schwangerschaft vorzeitige Wehen aufgetreten sind oder nicht. Die anderen Teilnehmerinnen hatten zu dieser Frage keine Angaben gemacht. Bei den Teilnehmerinnen, von denen Angaben zu vorzeitiger Wehentätigkeit (vorz. Wehen „ja“ oder „nein“) vorlagen $(n=549)$, gaben $n=423$ an, dass bei ihnen keine vorzeitigen Wehen aufgetreten sind und $\mathrm{n}=126$ gaben an, dass bei innen vorzeitige Wehen aufgetreten sind. Die Unterschiede wurden anschließend geprüft (Tab. A4 im Tabellenband).

Folgende signifikanten Unterschiede zwischen beiden Gruppen konnten gefunden werden:

- Untergewicht mit einem BMl <20 war bei Frauen mit vorzeitigen Wehen häufiger $(38,1 \%$ vs. $17,0 \%)$

- gynäkologische Komplikationen vor Beginn der Schwangerschaft waren bei Frauen mit vorzeitigen Wehen häufiger (19,0\% vs. 10,2\%);

- die Zeit des Weiterreitens während der Schwangerschaft war bei Frauen mit vorzeitigen Wehen kürzer (25,8 Wochen vs. 29,1 Wochen).

Die erhöhte Rate an Frühgeburten in der Gruppe mit vorzeitigen Wehen war trotz der geringen Fallzahl und trotz der hohen Standardabweichung signifikant $(13,5 \%$ [ $n=17]$ vs. 
7,3\% [n=31], T-Test 3,5). Ebenfalls signifikant war der Unterschied in der durchschnittlichen Dauer der Schwangerschaft: Bei den Reiterinnen mit vorzeitigen Wehen betrug die mittlere Schwangerschaftsdauer 38,9 Wochen, bei den Reiterinnen ohne vorzeitige Wehen 39,5 Wochen (Tab. 9).

Tab. 9. Reiterinnen mit und ohne vorzeitige Wehen (Primiparae, Einlinge).

\begin{tabular}{|c|c|c|c|c|c|c|}
\hline $\begin{array}{l}\text { Absolut Angaben zu } \\
\text { vorz. Wehen }(n=549)\end{array}$ & $\begin{array}{l}\text { PP ohne } \\
\text { vorz. } \\
\text { Wehen }\end{array}$ & Prozent & $\begin{array}{l}\text { PP mit } \\
\text { vorz. } \\
\text { Wehen }\end{array}$ & Prozent & T-Test & Sign. \\
\hline $\mathbf{N}$ & 423 & & 126 & & & \\
\hline Untergewicht & 72 & $17,0 \%$ & 48 & $38,1 \%$ & 2,6 & $\mathrm{Ja}$ \\
\hline \multirow[t]{2}{*}{ Gynäkologische Anamnese } & 43 & $10,2 \%$ & 24 & $19,0 \%$ & 1,0 & Nein \\
\hline & $\begin{array}{l}\text { PP ohne } \\
\text { vorz. } \\
\text { Wehen }\end{array}$ & $\begin{array}{l}\text { Mittelwert } \\
\text { I Wochen } \\
\text { (St.Abw) }\end{array}$ & $\begin{array}{l}\text { PP mit } \\
\text { vorz. } \\
\text { Wehen }\end{array}$ & $\begin{array}{l}\text { Mittelwert } \\
\text { (St.Abw)I } \\
\text { Wochen }\end{array}$ & T-Test & Sign. \\
\hline $\begin{array}{l}\text { Weiterreiten in der } \\
\text { Schwangerschaft bis Woche }\end{array}$ & 423 & $\begin{array}{l}29,1 \\
(9,0)\end{array}$ & 126 & $\begin{array}{l}25,8 \\
(9,5)\end{array}$ & 3,5 & $\mathrm{Ja}$ \\
\hline \multirow[t]{2}{*}{ Dauer der Schwangerschaft } & 423 & $\begin{array}{c}39,5 \\
(1,94)\end{array}$ & 126 & $\begin{array}{l}38,9 \\
(2,5)\end{array}$ & 2,5 & $\mathrm{Ja}$ \\
\hline & $\begin{array}{c}\text { PP ohne } \\
\text { vorz. } \\
\text { Wehen }\end{array}$ & Prozent & $\begin{array}{l}\text { PP mit } \\
\text { vorz. } \\
\text { Wehen }\end{array}$ & Prozent & T-Test & Sign \\
\hline Frühgeburt bis $36+6$ Wochen & 31 & $7,3 \%$ & 17 & $13,5 \%$ & 3,3 & Ja \\
\hline
\end{tabular}

Von den 126 Primiparae mit vorzeitigen Wehen gaben nur 15 an, dass sie nach Einsetzen der Wehentätigkeit mit dem Reiten aufgehört haben.

Die durchschnittliche Schwangerschaftsdauer war bei Frauen, die mit dem Reiten nach dem Auftreten von Wehen aufgehört haben, und bei denen, die weitergeritten sind, mit 38,9 Wochen identisch.

Der der von den Schwangeren subjektiv wahrgenommene Parameter „Vorzeitige Wehen“ eignet sich damit, um eine drohende Frühgeburt zu erkennen. Allerdings lässt sich mit den vorliegenden Daten nicht nachweisen, dass es die Dauer der Schwangerschaft verlängern kann, wenn die Schwangere nach dem Auftreten von vorzeitigen Wehen das Reiten einstellt. 


\subsection{Sekundäre Sektiones und vaginal-operative Entbindungen}

Um der Frage nachzugehen, ob Reiterinnen durch die sportliche Belastung eine rigidere Beckenbodenmuskulatur haben als Nicht-Reiterinnen, und ob dies unter der Geburt von klinischer Relevanz sein kann, wurde in dem Fragebogen nach „protrahiertem Geburtsverlauf oder Schwierigkeiten bei der Entbindung" gefragt und die Möglichkeit zum Freitext-Eintrag gegeben. Nach Dammrissen und nach Dammschnitten wurde nicht gefragt (siehe S. 17).

19,1\% der Primiparae und 4,8\% der Multiparae gaben einen „verlängerten Geburtsverlauf“ an. $32,8 \%$ bzw. 17,8\% gaben an „Schwierigkeiten bei der Entbindung“, und fast ebensoviele fügten im Freitext-Feld „Erläuterungen zu einem schwierigen Geburtsverlauf“ hinzu. Da es sich hier um subjektive, retrospektive Berichte der Teilnehmerinnen handelte und keine Vergleichsdaten vorliegen, werden diese Angaben für die Beantwortung der gestellten Frage nicht verwertet.

Im Folgenden werden die Parameter „sekundäre Sectio“ und „vaginal-operative Entbindung“ bei Einlingsgeburten als Anhaltspunkte für ein mögliches klinisch relevantes Geburtshindernis aufgrund der reiterlichen Aktivität herangezogen und den Daten aus der niedersächsischen Perinatalerhebung gegenübergestellt (Tab. 10).

Tab. 10. Geburtsmodus in der Reiterinnenstudie im Vergleich zur Niedersächsischen Perinatalstudie, Primi- und Multiparae.

\begin{tabular}{|l|c|c|}
\hline Entbindungsmodus & $\begin{array}{c}\text { Niedersächs. Perinat- } \\
\text { Studie 2004, Einlinge, PP } \\
\mathbf{n = 6 4 . 6 9 2}\end{array}$ & $\begin{array}{c}\text { Reiterinnen-Studie } \\
\text { Einlinge, PP und MP } \\
\mathbf{n = 1 . 5 5 8}\end{array}$ \\
\hline Spontan & $67,3 \%$ & $67,1 \%$ \\
\hline Kaiserschnitt insgesamt & $26,8 \%$ & $23,9 \%$ \\
\hline Geplanter Kaiserschnitt & $14,6 \%$ & $10,1 \%$ \\
\hline Ungeplanter Kaiserschnitt & $12,2 \%$ & $13,8 \%$ \\
\hline Saugglocke oder Zange & $5,7 \%$ & $9,0 \%$ \\
\hline
\end{tabular}

In der Reiterinnenstudie liegt der Anteil der Primiparae mit $72 \%$ deutlich höher als bei der Perinatalstudie mit 47\%, der Anteil der Multiparae mit 28\% deutlich niedriger. Deshalb wurde die Analyse des Geburtsmodus im Vergleich zur Niedersächsischen Perinatalstudie getrennt für Primi- und Multiparae durchgeführt (Tab. 11 und Tab. 12). Bei der Analyse der Geburtsmodi getrennt für Erst- und Mehrgebärende handelt es sich um unveröffentlichtes 
Datenmaterial der Niedersächsischen Perinatalerhebung aus dem Jahr 2003 (Wenzlaff 2010).

Tab. 11. Geburtsmodus bei Primiparae. Reiterinnen im Vergleich zur Niedersächsischen Perinatalstudie (NDS). Konfidenzintervalle und signifikante Differenzen.

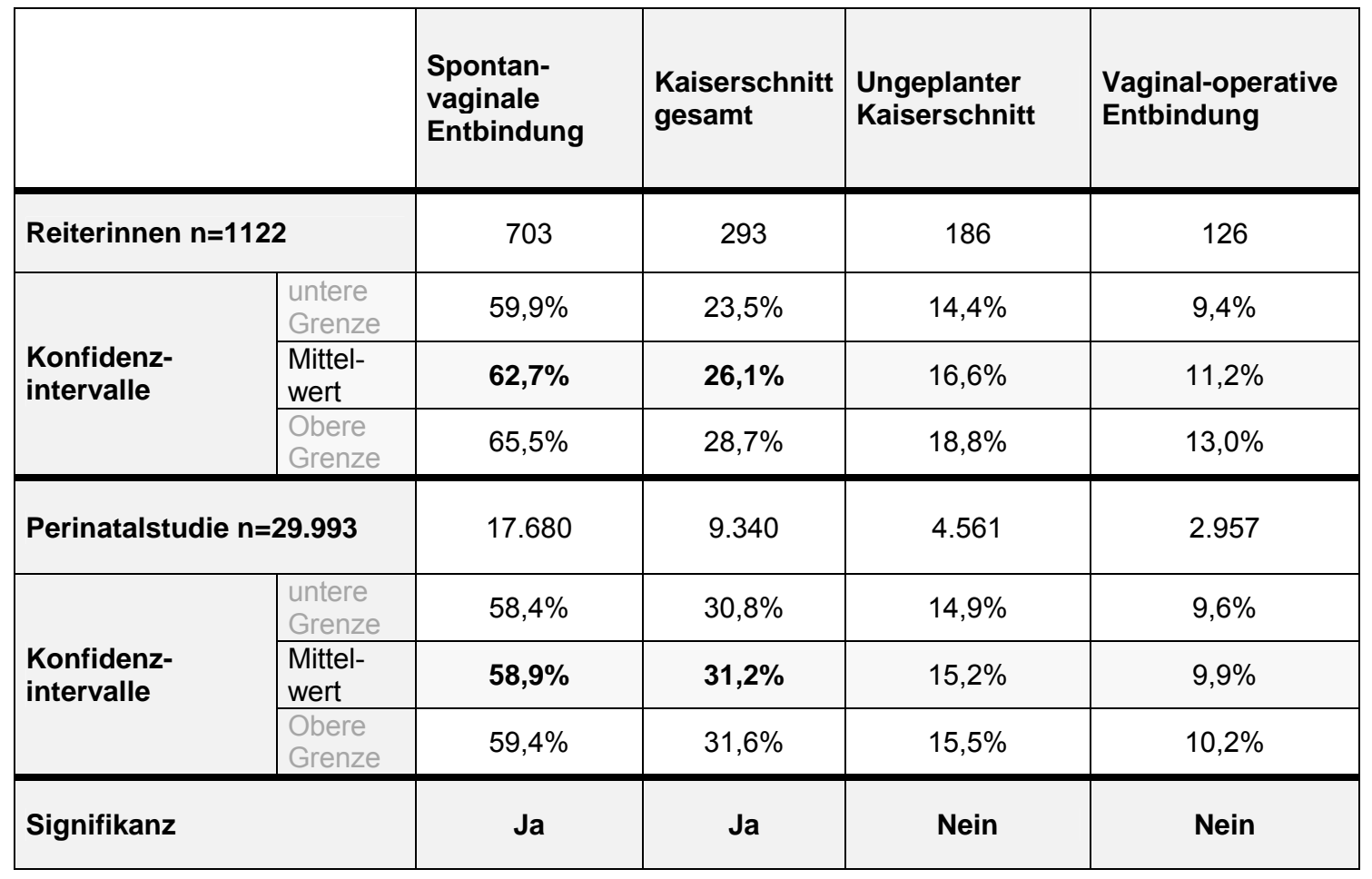

Tab. 12. Geburtsmodus bei Multiparae. Reiterinnen im Vergleich zur Niedersächsischen Perinatalstudie (NDS). Konfidenzintervalle und signifikante Differenzen.

\begin{tabular}{|c|c|c|c|c|c|}
\hline & & $\begin{array}{l}\text { Spontan- } \\
\text { vaginale } \\
\text { Entbindung }\end{array}$ & $\begin{array}{l}\text { Kaiserschnitt } \\
\text { gesamt }\end{array}$ & $\begin{array}{l}\text { Ungeplanter } \\
\text { Kaiserschnitt }\end{array}$ & $\begin{array}{l}\text { Vaginal-operative } \\
\text { Entbindung }\end{array}$ \\
\hline \multicolumn{2}{|c|}{ Reiterinnen $n=436$} & 342 & 80 & 29 & 14 \\
\hline \multirow{3}{*}{$\begin{array}{l}\text { Konfidenz- } \\
\text { intervalle }\end{array}$} & $\begin{array}{l}\text { untere } \\
\text { Grenze }\end{array}$ & $74,5 \%$ & $14,7 \%$ & $4,4 \%$ & $1,5 \%$ \\
\hline & $\begin{array}{l}\text { Mittel- } \\
\text { wert } \\
\end{array}$ & $78,4 \%$ & $18,3 \%$ & $6,7 \%$ & $3,2 \%$ \\
\hline & $\begin{array}{l}\text { Obere } \\
\text { Grenze }\end{array}$ & $82,3 \%$ & $21,9 \%$ & $9,0 \%$ & $4,9 \%$ \\
\hline \multicolumn{2}{|c|}{ Perinatalstudie $n=34.797$} & 17.680 & 9.340 & 4.561 & 2.957 \\
\hline \multirow{3}{*}{$\begin{array}{l}\text { Konfidenz- } \\
\text { intervalle }\end{array}$} & $\begin{array}{l}\text { untere } \\
\text { Grenze }\end{array}$ & $74,8 \%$ & $22,4 \%$ & $6,1 \%$ & $1,8 \%$ \\
\hline & $\begin{array}{l}\text { Mittel- } \\
\text { wert }\end{array}$ & $75,2 \%$ & $22,8 \%$ & $6,3 \%$ & $1,9 \%$ \\
\hline & $\begin{array}{l}\text { Obere } \\
\text { Grenze }\end{array}$ & $75,6 \%$ & $23,2 \%$ & $6,5 \%$ & $2,0 \%$ \\
\hline \multicolumn{2}{|l|}{ Signifikanz } & Nein & $\mathrm{Ja}$ & Nein & Nein \\
\hline
\end{tabular}


Nach dieser Auswertung haben die Reiterinnen in der Studie im Vergleich zu den Daten der Niedersächsischen Perinatalstudie

- einen signifikant höheren Anteil an spontan-vaginalen Entbindungen bei der ersten Entbindung,

- einen signifikant verringerten Anteil an Sectiones bei Primi- und Multiparität,

- keinen signifikant erhöhten Anteil an sekundären Sectiones und an vaginal-operativen Entbindungen.

\section{Fazit}

Ein erhöhter Anteil an vaginal-operativer Entbindungen und an sekundären Kaiserschnitten, die unter anderem als klinisch relevante Parameter für kritisch protrahierte Geburtsverläufe interpretiert werden können, kann nach einem Vergleich mit der Niedersächsischen Perinatalerhebung nicht nachgewiesen werden. 


\subsection{Vaginal-operative Entbindungen in Abhängigkeit von der reiterlichen Aktivität}

Um der Frage im Detail nachzugehen, ob bei trainierten Reiterinnen die Beckenbodenmuskulatur so fest werden kann, dass sie zu einem klinisch relevanten Geburtsstillstand mit konsekutiver vaginal-operativer Entbindung führen könnte, werden aus der Gruppe der 1122 Primiparae die Teilnehmerinnen mit spontaner vaginaler Entbindung $(n=703)$ und diejenigen mit vaginal-operativer Entbindung $(n=126)$ verglichen.

Wenn es sich um einen trainingsbedingten Effekt handeln sollte, so müsste sich eine Abhängigkeit vom aktuellen Trainingszustand der Reiterin, von der Intensität und Dauer des Reitens während der Schwangerschaft nachweisen lassen.

Die Analyse (siehe Tab. A7A im Tabellenband) zeigt, dass beide Gruppen - spontane Entbindung und vaginal-operative Entbindung - keinerlei Unterschiede aufweisen, vor allem nicht hinsichtlich

- ihres allgemeinen sportlichen Trainingszustandes vor und während der Schwangerschaft,

- der Dauer des Weiterreitens während der Schwangerschaft (28,8 vs. 29,7 Wochen),

- der Zahl der Stunden im Sattel pro Tag und pro Woche (siehe Abb. 8),

- der reiterlichen Disziplinen vor und während der Schwangerschaft (Dressur, Springen, Gelände, siehe Abb. 9).

Die Abbildungen - die Daten wurden aus Tab. A7A im Tabellenband entnommen - zeigen exemplarisch, dass die reiterlichen Trainingszeiten pro Woche in beiden Gruppen weitgehend identisch sind, ebenso die reiterlichen Disziplinen und Ambitionen während der Schwangerschaft.

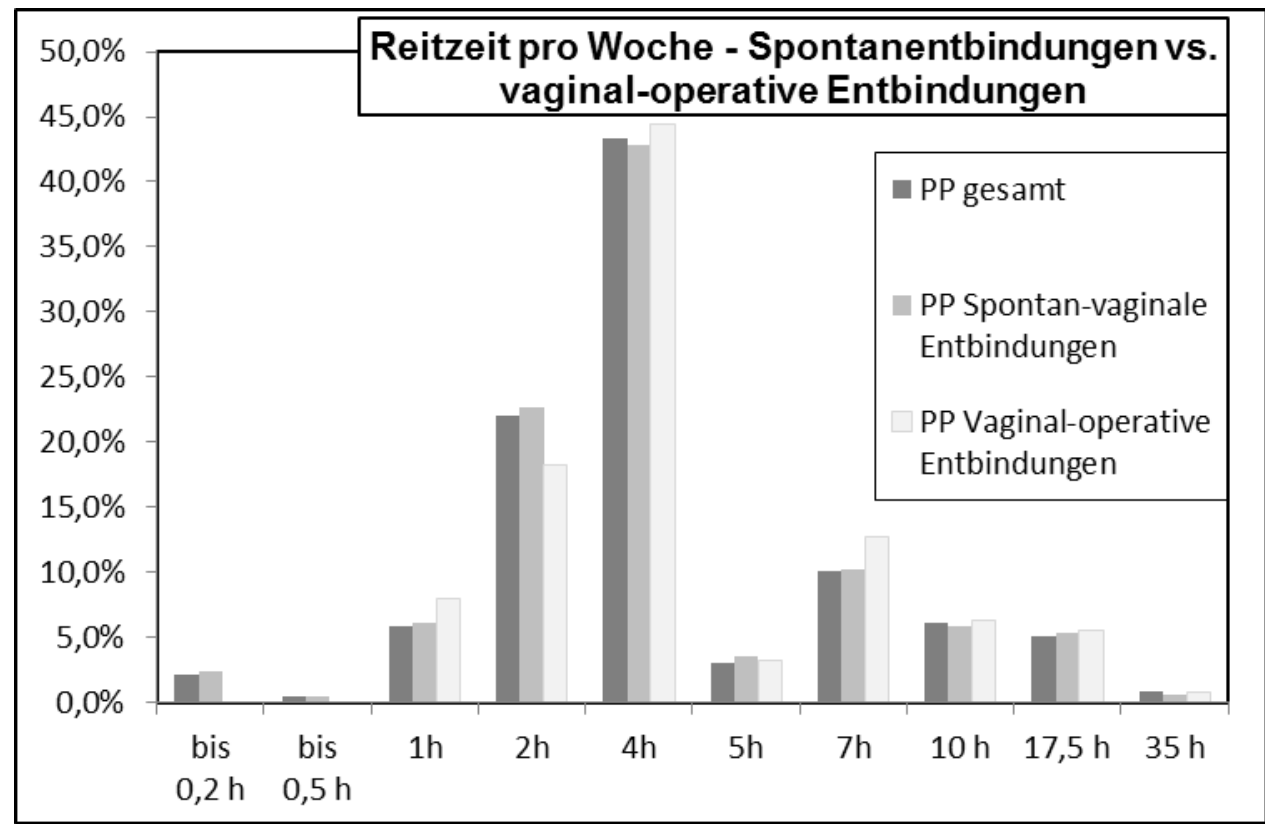

Abb. 8. Reiterliche Trainingszeit pro Woche - Spontanentbindungen vs. vaginal-operative Entbindungen. 


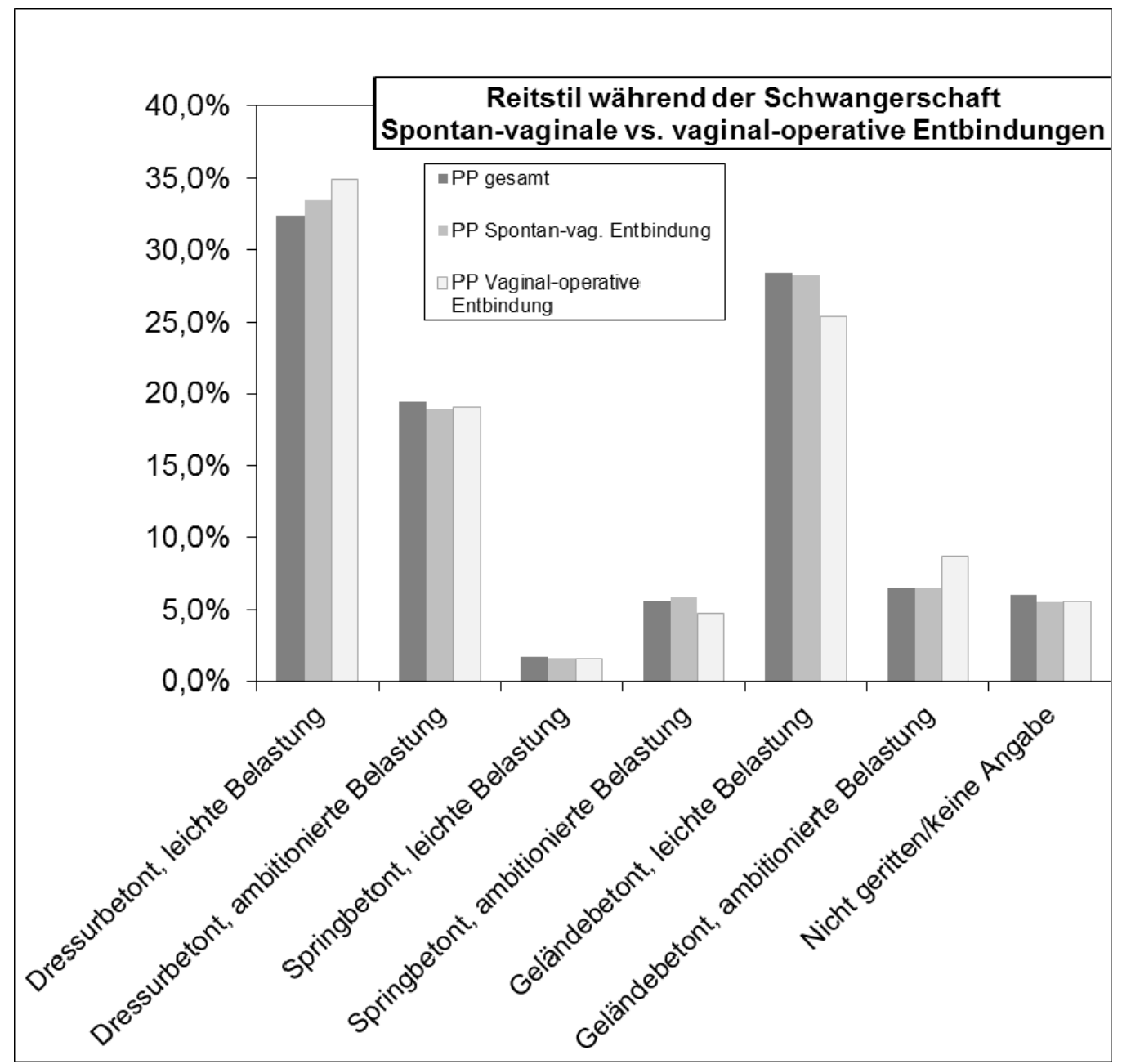

Abb. 9.

Reitstil

während der Schwanger-

schaft -

Spontan-

vaginale vs.

vaginal-

operative

Entbin-

dungen.

Die durchschnittliche Dauer der Schwangerschaft ist bei den Teilnehmerinnen mit vaginaloperativer Entbindung um 0,4 Wochen länger (39,9 vs. 39,4 Wochen), die Kinder im Durchschnitt $100 \mathrm{~g}$ schwerer und $1 \mathrm{~cm}$ länger als bei den Frauen mit Spontangeburt. Diese Unterschiede sind nicht signifikant, deuten aber darauf hin, dass eher akute geburtshilfliche Fragestellungen zur Geburtsbeendigung per Saugglocke oder Zange geführt haben als eine Beckenbodenmuskulatur, die durch reiterliches Training zu einem klinisch relevanten Geburtshindernis geworden wäre.

Vergleicht man zusätzlich diejenigen Entbindungen, die mit einer sekundären Sectio beendet wurden, mit denen, die mit Spontangeburt beendet wurden, so ergibt sich dasselbe Bild: In Bezug auf die Dauer des Weiterreitens, die wöchentliche Reitstundenzahl während der Schwangerschaft, die reiterlichen Disziplinen und Ambitionen gibt es zwischen diesen Gruppen keinerlei Unterschiede (Tabellenband Tab. A7B).

Was die nicht-gynäkologisch-geburtshilflichen Parameter angeht, so unterscheiden sich die Teilnehmerinnen mit sekundären Sectiones lediglich dadurch, dass in dieser Gruppe im Vergleich zu Frauen Spontanentbindung häufiger mütterliches Übergewicht und Bluthochdruck 
auftritt, ein höherer Anteil an Geburten am oder über dem Termin, schwerere Kinder und häufigere Beckenendlagen (Tabellenband Tab. A7B).

\subsection{Geburt aus Beckenendlage}

Weiterhin wurde anhand der vorliegenden Daten die Häufigkeit von Beckenendlagen bei Reiterinnen geprüft. Grund für diese Überprüfung war die Vorstellung, dass es dem Feten möglicherweise unangenehm sein müsse, das Reiten der Mutter in Schädellage miterleben und die reiterlichen Bewegungen mit Kopf und Genick abfedern zu müssen, so dass er deswegen bestrebt sein könnte, eine Beckenendlage einzunehmen, um so die biometrischen Belastungen in aufrechter Lage mit dem Gesäß nach unten besser zu tolerieren. Zu dieser Hypothese gibt es keinerlei Literatur, auch nicht aus anderen Sportarten.

Eine Beckenendlage stellt nach den Mutterschaftsrichtlinien ein Schwangerschafts- und Geburtsrisiko dar.

Deswegen wurden die vorliegenden Daten auf Hinweise untersucht, dass das Reiten während der Schwangerschaft, insbesondere intensives Reiten bis zum Geburtstermin hin oder dressurmäßiges Reiten im tiefen Sitz, das Risiko für eine Beckenendlage erhöht.

\subsubsection{Schädellage vs. Beckenend- und Querlage - Häufigkeiten}

In der Untersuchung finden sich folgende Häufigkeiten für Geburt aus Schädel- bzw. Beckenend- oder Querlage (Tab. 13):

- Bei den 1558 gültigen Fragebögen (Einlingsentbindungen) ist die Kindslage in 434 Fällen nicht angegeben.

- Die durchschnittliche Schwangerschaftsdauer bei Frauen, bei denen die Kindslage unbekannt ist, liegt bei 39,4 Wochen (StAbw. 1,94). Bei Frauen, denen die Kindslage bekannt ist, liegt die Schwangerschaftsdauer bei 39,7 Wochen (StAbw. 1,86). Die Differenz ist signifikant (T-Test 2,8).

- Von den 1124 Entbindungen mit bekannter Kindslage entfallen 1050 (93,4\%) auf Schädellagen und 74 (6,6\%) auf Beckenend-, Quer- und Schräglagen.

- Die durchschnittliche Schwangerschaftsdauer betrug bei Schädellage 39,7 Wochen (StAbw 1,77), bei BEL und Querlage 38,7 Wochen (StAbw 1,88). Die Differenz zwischen beiden Werten ist signifikant $(p<0,05)$. 
Tab 13. Bekannte Kindslagen bei Entbindung.

\begin{tabular}{|l|c|c|c|c|c|}
\hline & $\begin{array}{l}\text { Alle Teil- } \\
\text { nehme- } \\
\text { rinnen }\end{array}$ & $\begin{array}{l}\text { Kindslage } \\
\text { unbekannt }\end{array}$ & $\begin{array}{l}\text { Kindslage } \\
\text { bekannt }\end{array}$ & $\begin{array}{l}\text { Schädellage } \\
\text { (\% der bekannten } \\
\text { Kindslagen) }\end{array}$ & $\begin{array}{l}\text { BEL/Querlage } \\
\text { (\% der bekann- } \\
\text { ten Kindslagen) }\end{array}$ \\
\hline $\mathrm{n}$ & 1558 & 434 & 1124 & $1050(93,4 \%)$ & $74(6,6 \%)$ \\
\hline $\begin{array}{l}\text { Dauer der } \\
\text { Schwangerschaft } \\
\text { in Wochen }\end{array}$ & 39,6 & 39,4 & 39,7 & 39,7 & 38,7 \\
\hline $\begin{array}{l}\text { Standard- } \\
\text { abweichung }\end{array}$ & 1,83 & $1,94^{*}$ & $1,86^{*}$ & $1,77^{*}$ & $1,88^{*}$ \\
\hline T-Test & & \multicolumn{3}{|c|}{2,8} & \\
\hline
\end{tabular}

\subsubsection{Vergleich Entbindungen aus Schädellage vs. Beckenend- und Querlage}

Unter den Schwangerschaften, die aus BEL/Querlage entbunden werden, finden sich im Vergleich zu denen die aus Schädellage entbunden werden, signifikante Unterschiede $(p<0,05)$ bei folgenden Parametern (Tab. A5 im Tabellenband):

- Komplikationen bei früheren Schwangerschaften und Entbindungen (47,3\% bei BEL vs. $32,9 \%$ bei $S L)$,

- $\quad$ sportliche Aktivität zusätzlich zum Reiten ( $71,6 \%$ bei BEL vs. $60,2 \%$ bei $S L)$, wobei verstärkt ausdauerbetonte Sportarten angegeben werden (55,4\% vs. $41,2 \%)$;

- dressurbetontes Reiten VOR der Schwangerschaft (95,9\% bei BEL vs. $84,0 \%$ bei SL) steht in einem deutlichen Zusammenhang mit einer Entbindung aus BEL, vor allem leistungsorientiertes Reiten (59,5\% bei BEL vs. $44,5 \%$ bei $S L)$;

- dressurbetontes Reiten WÄHREND der Schwangerschaft $(82,4 \%$ bei BEL vs. $72,0 \%$ bei $S L)$ stellt ebenfalls ein Risiko dar, dass die Schwangerschaft in einer BEL endet. Hier ist kein Zusammenhang mit der Intensität des dressurbetonten Reitens erkennbar;

- Entbindung per Sectio caesarea ( $81,1 \%$ bei BEL vs. $18,7 \%$ bei $S L)$.

\subsubsection{Schädellage vs. Beckenend- und Querlage - weitere Unterschiede}

Nicht signifikante Unterschiede zwischen beiden Gruppen lassen sich bei folgenden Parametern finden:

- Parität (Anteil von Primiparae von $78,4 \%$ bei BEL vs. $70,7 \%$ bei $S L$ )

- Frühgeburtlichkeit $(9,5 \%$ bei BEL vs. $5,1 \%$ bei $S L)$, 


\subsubsection{Schädellage vs. Beckenend- und Querlage - nicht unterschiedliche Parameter}

Bei allen anderen erhobenen Parametern lässt sich kein Einfluss auf die Kindslage bei Entbindung feststellen, insbesondere nicht bei

- Dauer des Reitens pro Woche während der Schwangerschaft,

- reiterlichen Ambitionen und Leistungsorientierung während der Schwangerschaft,

- Spring- und Geländereiten,

- Dauer des Weiterreitens in der Schwangerschaft ( $\varnothing$ SSW 28,1 bei BEL, $\varnothing 29,1$ bei $S L)$,

- $\quad$ sonstiger sportlicher Betätigung während der Schwangerschaft.

Eine Beeinträchtigung des kindlichen Outcome durch die Entbindung aus BEL kann nicht festgestellt werden (Kind gesund geboren in 95,9\% aus BEL, in 95,2\% aus SL, n.s.).

\subsubsection{Kindslage beim Reiten bis zur Entbindung am Termin}

Der Fet ändert seine Lage ständig bis etwa zur 37. Woche. Deshalb müsste die Hypothese, dass das Reiten in der Schwangerschaft das Risiko einer Beckenendlage erhöht, geprüft werden können, indem man die Kindslagen bei denjenigen Schwangeren betrachtet, die bis zur Terminnähe geritten sind. Denn der Fet würde sich in der Spätschwangerschaft aus der BEL, die er eventuell bei den reiterlichen Aktivitäten seiner Mutter eingenommen hat, nicht mehr in die SL zurückdrehen können.

Es müssten sich dann bei Frauen, die bis zur Geburt nach der 37. Woche weitergeritten sind, mehr Beckenendlagen finden als bei Frauen, die deutlich früher mit dem Reiten aufgehört haben.

173 Frauen im untersuchten Kollektiv sind bis über $37+0$ Wochen hinaus geritten. In diesem Kollektiv finden sich 168 Schädellagen (97,1 \%) und 5 BEL/Querlagen (2,9\%) (Tab. 14).

Tab. 14. Kindslage bei Schwangeren, die ab 37+0 entbunden haben und bis zum ET weitergeritten sind bzw. bei Schwangeren, die höchstens bis 33+0 SSW geritten sind.

\begin{tabular}{|c|c|c|c|c|c|c|}
\hline & \multicolumn{2}{|c|}{ Geritten bis zum ET } & \multicolumn{2}{|c|}{$\begin{array}{l}\text { Mit dem Reiten } \\
\text { aufgehört bis 33+0 } \\
\text { SSW }\end{array}$} & \multirow[t]{2}{*}{ T-Test } & \multirow[t]{2}{*}{ Signifik. } \\
\hline $\begin{array}{l}\text { Entbindung am Termin (ab 37+1 } \\
\text { ssW) }\end{array}$ & 173 & $100 \%$ & 814 & $100 \%$ & & \\
\hline Entbindung aus SL & 169 & $97,1 \%$ & 759 & $93,2 \%$ & 2,51 & $\mathbf{s}$ \\
\hline Entbindung aus BEL/QL & 5 & $2,9 \% *$ & 54 & $6,6 \% *$ & 2,40 & $s$ \\
\hline
\end{tabular}


Wir stellen diese Gruppe denjenigen Frauen gegenüber, die nach $37+0$ Wochen entbunden haben, aber spätestens mit Beginn der Schwangerschaftswoche 33 mit dem Reiten aufgehört haben. In diesem Kollektiv finden sich 759 Schädellagen (93,2\%) und 54 Beckenend- bzw. Querlagen (6,6\%).

Dies bedeutet, dass die Schwangeren, die bis zur Geburt am ET weiterreiten, signifikant seltener aus Beckenend- oder Querlage entbunden haben als Schwangere, die höchstens bis zur 33. Woche weitergeritten sind.

Eine vergleichende Analyse der beiden Gruppen (Tab. A6 im Tabellenband) zeigt, dass zwischen den beiden Gruppen keinerlei Unterschiede in der geburtshilflichen, der gynäkologischen, der reiterlichen Anamnese und dem Verlauf der Schwangerschaft gefunden werden können, die erklären könnten, wieso sich in der Gruppe der Frauen, die frühzeitig mit dem Reiten aufhören, mehr BEL/QL-Entbindungen finden als bei denen, die bis zum ET weiterreiten.

Bei den Frauen, die bis zum ET weiterreiten, findet sich ein signifikant höherer Anteil von Berufsreiterinnen, die 7h und mehr pro Woche reiten, ebenso ein signifikant höherer Anteil von Reiterinnen, die auch während ihrer Schwangerschaft im leistungsorientierten Sport und im Spitzensport reiten und intensiv Dressur reiten.

Damit kann eine Beeinflussung der Kindslage durch reiterliche Belastung allgemein während der Schwangerschaft mit den vorliegenden Daten nicht nachgewiesen werden. Dressurbetontes Reiten vor oder während der Schwangerschaft erhöht das Risiko für eine Entbindung aus Beckenendlage geringfügig, aber nicht signifikant. 


\subsection{Unfälle beim Reiten während der Schwangerschaft}

Unter den 1558 für diese Fragestellung herangezogenen Datensätzen (Primi- und Multiparae) finden sich 151 Teilnehmerinnen mit Unfällen beim Reiten oder beim Umgang mit dem Pferd (9,7\%). Alle von den Reiterinnen selbst angegebenen Ereignisse wurden in die Auswertung mit einbezogen, unabhängig davon, ob sie zu einer Vorstellung beim Arzt geführt haben oder nicht (Tab. A8 im Tabellenband).

Kasuistisch berichtet eine der Teilnehmerinnen über eine Fehlgeburt in der 23. Woche nach einem Unfall, eine weitere über eine Frühgeburt in der 33. Woche nach einem Unfall, wobei das Kind nicht geschädigt wurde (gesund nach 1 Woche und nach 1 Jahr).

Abgesehen von den beiden o.g. Einzelfällen haben die Unfälle bei 15\% der Schwangeren zu vorzeitigen Wehen geführt, die aber beherrscht werden konnten: Die Schwangerschaftsdauer ist mit 39,6 Wochen bei Reiterinnen mit und ohne Unfällen identisch.

In der Gruppe der Reiterinnen mit Unfällen (siehe Abb. 10) finden sich im Vergleich mit den Frauen ohne Unfälle signifikant häufiger $(p<0,05)$

- Frauen, die bereits Unfallfolgen aus früheren Reitunfällen berichten (11,3\% vs. 2,0\%),

- Reiterinnen, die in der Selbsteinschätzung „Reiten mit gewissem Unfallrisiko“ angeben $(13,2 \%$ vs. $3,8 \%)$,

- Reiterinnen, die junge Pferde oder Problempferde geritten haben (47,0\% vs. $21,5 \%)$,

- Reiterinnen im Spitzensport während der Schwangerschaft (6,6\% vs. 2,5\%),

- Reiterinnen mit einer durchschnittlichen Reitzeit von $2 \mathrm{~h}$ und mehr pro Tag (14 h pro Woche) auch in der Schwangerschaft $(9.9 \%$ vs. $4,2 \%)$,

- Reiterinnen mit ambitioniertem Springtraining während der Schwangerschaft (16,6\% vs. $8,2 \%)$,

- bevorzugtes Reiten von Privat- und Ausbildungspferden in der Schwangerschaft $(19,5 \%$ vs. $9,3 \%)$.

Ein additiver Effekt der oben genannten Risikofaktoren lässt sich nicht feststellen, da es sich immer um die gleiche Hochrisikogruppe handelt: Professionelle Reiterinnen mit einer hohen Reitleistung pro Woche, zu deren Aufgabenbereich die Ausbildung junger Pferde ebenso wie forciertes Springtraining gehört. 
Folgende nicht-reiterlichen Unterschiede zwischen der Gruppe der Frauen ohne und mit Unfällen während der Schwangerschaft sind signifikant:

- Starke Belastung durch den Zwang zu schnellen Entscheidungen (13,2\% vs. 3,8\%),

- $\quad$ starke Belastung durch Zwang zu hoher Konzentrationsleistung (9,9\% vs. 4,7\%).

Abb. 10. Unfallrisiken beim Reiten oder beim Umgang mit dem Pferd während der Schwangerschaft.

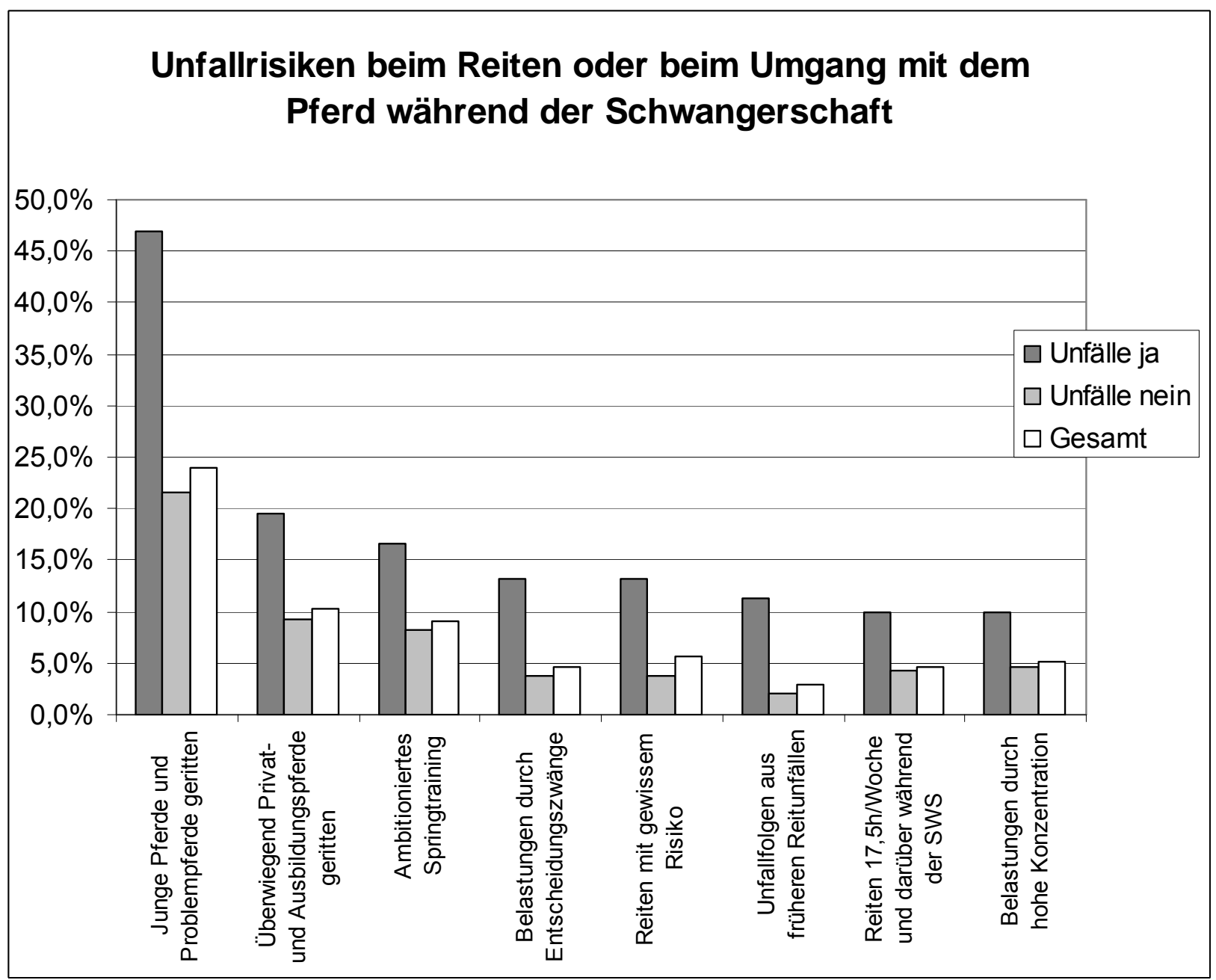

Leichtes Springtraining erhöhte das Unfallrisiko nicht.

Folgende schwach signifikanten Unterschiede zwischen den beiden Gruppen fallen zusätzlich auf $(p<0,1)$ :

- Turnierteilnahmen während der Schwangerschaft (27,8\% vs. $18,6 \%)$.

- $\quad$ starke Belastung durch Beruf, Familie oder anderes (35,1\% vs. $22,7 \%$ ),

- häufige Belastung durch Beruf, Familie oder anderes (32,5\% vs. $24,9 \%)$. 
97\% der Reiterinnen, die detaillierte Angaben zu Unfallhergang und -häufigkeiten gemacht haben, gaben Stürze vom Pferd an, 41\% Stürze mit dem Pferd, 57,7\% Unfälle beim Umgang mit dem Pferd (Abb. 11).

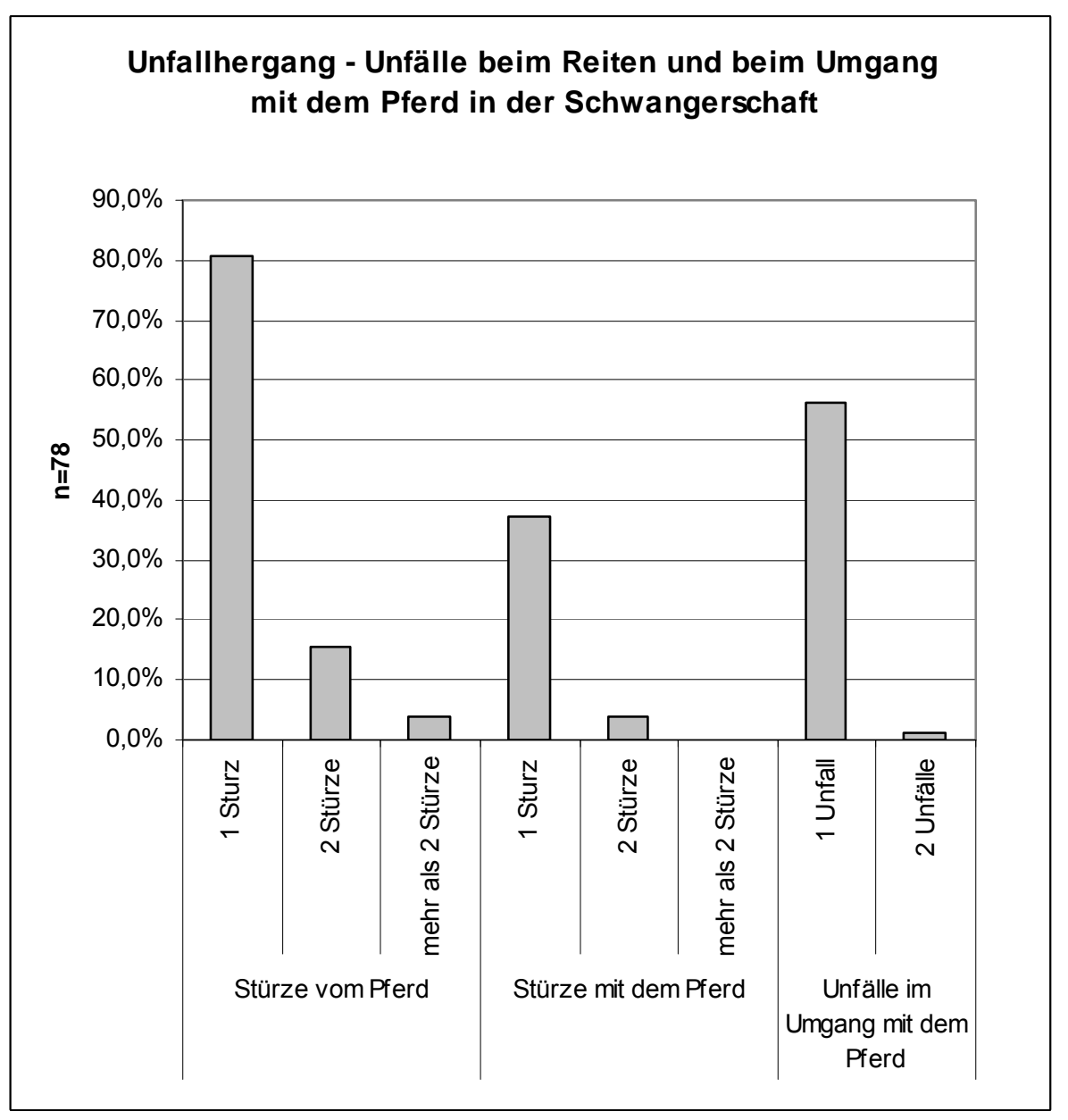

Abb. 11. Unfallhergang und -häufigkeiten beim Reiten und beim Umgang mit dem Pferd in der Schwangerschaft ( $n=78$ Reiterinnen mit Angaben zu Unfallhergang und häufigkeiten).

Allerdings gibt ein vorsichtiger Reitstil keine völlige Sicherheit für ein unfallfreies Reiten:

- $\quad 74,8$ \% der Reiterinnen mit Unfällen sind in der Schwangerschaft keine Hindernisse gesprungen,

- $72,2 \%$ der Reiterinnen mit Unfällen nahmen nicht an Turnieren teil,

- $66,9 \%$ der Reiterinnen mit Unfällen schlossen sich im Fragebogen der Aussage an „Ich fühlte mich völlig sicher, ein Unfallrisiko war aus meiner Sicht ausgeschlossen“;

- $53 \%$ der Reiterinnen mit Unfällen haben keine jungen Pferde und keine Problempferde geritten (Abb. 12). 


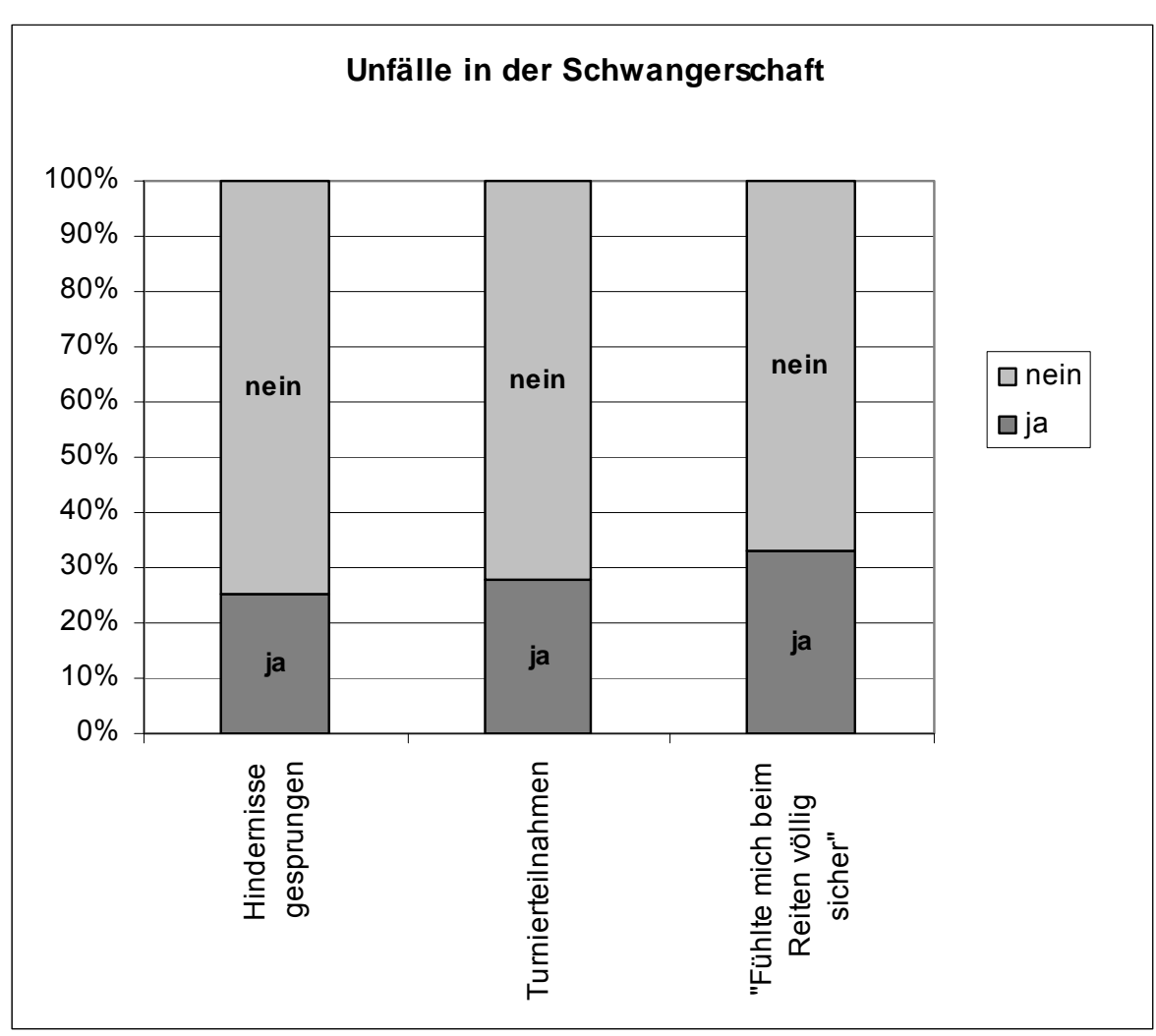

Abb. 12. Reitunfälle in der Schwangerschaft Unfallrisiken und Selbsteinschätzungen.

\section{Fazit}

9,7\% der Studienteilnehmerinnen geben einen oder mehrere Unfälle beim Reiten oder beim Umgang mit dem Pferd während der Schwangerschaft an.

Unfälle beim Reiten oder beim Umgang mit dem Pferd verkürzen die durchschnittliche Schwangerschaftsdauer nicht.

Besonders gefährdet sind erfahrene, professionelle und ambitionierte Reiterinnen, Reiterinnen im Spitzensport und Reiterinnen im gehobenen Springsport. Das höchste Risiko stellt der Umgang mit jungen Pferden und mit Problempferden dar.

Hohe Stressbelastung und Bluthochdruck während der Schwangerschaft können als Indikatoren für eine erhöhte Unfallgefahr angesehen werden.

Jedoch können auch Reiterinnen ohne Springtraining, ohne Umgang mit Risikopferden und ohne erhöhte Stressfaktoren Unfälle beim Reiten oder beim Umgang mit dem Pferd nicht sicher vermeiden.

Fehl- und Frühgeburten als Unfallfolge können nicht ausgeschlossen werden. 


\section{Diskussion}

\subsection{Material und Methoden}

\section{$\underline{5.1 .1 \text { Diskussion Material }}$}

Ziel der Arbeit war es herauszuarbeiten, ob das Reiten als Sportart aufgrund seiner sportarttypischen Belastungsmuster einen Einfluss auf Schwangerschaft, Entbindung und das kindliche Outcome hat, und ob Unterschiede zu finden sind zwischen verschiedenen reiterlichen Disziplinen, der Intensität und Ambition des Reitens in der Schwangerschaft und der Dauer des Reitens während der Schwangerschaft.

Als Parameter, um diese Fragen zu beantworten, wurden Aborthäufigkeit, Frühgeburtlichkeit, die Häufigkeit von vaginal-operativen Entbindungen, sekundären Sektiones und von Entbindungen aus Beckenendlage untersucht.

Insgesamt haben reiterliche Aktivität und auch reiterliche Unfälle keinen oder einen nur sehr geringen Einfluss auf die Dauer der Schwangerschaft, den Geburtsverlauf und das kindliche Outcome. Allerdings ist zu beobachten, dass Frauen mit vorzeitigen Wehen oder Blutungen durchschnittlich viel früher mit dem Reiten aufhören als Frauen ohne Anzeichen von Frühgeburtsbestrebungen. Das Reiten in der Schwangerschaft kann damit als ein durch die Schwangere selbst kontrollierbares und kontrolliertes Risiko eingeschätzt werden.

Prospektive Untersuchungen und vergleichende Studien mit Schwangeren in anderen Sportarten sind daher wünschenswert.

\section{$\underline{5.1 .2}$ Diskussion der Methode}

Die Fragestellung wurde mit Hilfe einer retrospektiven Online-Befragung mit 54 MultipleChoice-Fragen und zahlreichen Möglichkeiten der individuellen Kommentierung durchgeführt. Sie umfasste 54 Fragen und im Ausdruck 8 Druckseiten (siehe Anhang). Ihre Beantwortung setzte Erfahrung im Umgang mit Multiple-Choice-Befragungen, mit einer großen Textfülle und ein zeitliches Engagement von etwa 20 Minuten voraus.

Diese Voraussetzungen könnten eine Selektion der Teilnehmerinnen verursacht und Teilnehmerinnen ausgeschlossen haben, die diese Anforderungen nicht erfüllen konnten oder wollten. Hierzu könnten Frauen mit niedrigem Bildungsabschluss ebenso gehören wie 
Frauen unter erheblichem Zeitdruck (mehrere Kinder im Haushalt und zusätzlich reiterliche Verpflichtungen). Dies könnte eine Erklärung dafür liefern, warum 57\% der Teilnehmerinnen einen Hochschulabschluss aufwiesen, was nicht dem Bevölkerungsdurchschnitt und auch nicht dem durchschnittlichen Bildungsniveau von gleichaltrigen Reiterinnen entspricht, und warum der Anteil der Multiparae mit 27\% viel geringer war als zu erwarten.

Um auszuschließen, dass die Ergebnisse durch die nicht repräsentative Verteilung von Schulbildung und Parität verzerrt wurden, wurde eine Gewichtung durchgeführt, die auch berücksichtigte, dass bei den Reiterinnen die Verteilung auf die Altersgruppen von der in der Niedersächsischen Perinatalstudie abwich.

Für die zentrale Fragestellung nach dem Zusammenhang zwischen Frühgeburtlichkeit und reiterlichem Engagement wurden ausschließlich Primiparae herangezogen.

Die retrospektive Untersuchung und die Beantwortung der Fragen durch die Teilnehmerinnen selbst barg die Gefahr, dass unerwünschte Ereignisse in der Schwangerschaft oder bei der Geburt überbetont und überinterpretiert wurden und dass sich Reiterinnen mit ungünstigem Ausgang ihrer Schwangerschaft häufiger beteiligt haben als Reiterinnen mit unauffälliger Schwangerschaft. Dieser Bias ist nicht auszuschließen. Würde man dieser Annahme folgen, so würde dies aber den Schluss nahelegen, dass in einer „Normalpopulation“ von schwangeren Reiterinnen Aborte, Frühgeburten und Unfälle eher noch seltener auftreten müssten als in der hier vorgelegten Untersuchung. Dieser Bias spielte in der Interpretation der Ergebnisse deshalb keine Rolle.

Hinsichtlich der reiterlichen Qualifikation wurde nach Leistungsklassen entsprechend der Einteilung der Deutschen Reiterlichen Vereinigung gefragt. Zusätzlich war die Möglichkeit zur Beantwortung in einem Textfeld gegeben, um auch Klassifizierungen außerhalb des Bewertungssystems der Deutschen Reiterlichen Vereinigung zuzulassen (Western-, Islandpferde-, Distanzreiter etc). Dies hat in vielen Fällen zu Unklarheiten und zu offensichtlich unplausiblen Antworten geführt, so dass dieses Kriterium in der Auswertung nicht berücksicht wurde.

\section{$\underline{\text { 5.1.3 Diskussion Repräsentativität }}$}

Die Frage, inwieweit die Teilnehmerinnen an der Studie repräsentativ sind für alle schwangeren Reiterinnen, kann nicht beantwortet werden. Die Öffentlichkeitsarbeit hatte vier Standbeine: 
- Zum einen eine eigene Projekt-Homepage mit hochrangiger Suchmaschinen-Präsenz und ausführlichen Informationen zur Studie, einem Diskussionsboard und dem Online-Fragebogen, der die Ergebnisse direkt in eine Datenbank einspeiste,

- zweitens die Öffentlichkeitsarbeit über die Deutsche Reiterliche Vereinigung und Reiterzeitschriften;

- das dritte Standbein waren Tageszeitungen und Publikumszeitschriften. Hier konnten allerdings trotz intensiver Kontakte zu den Redaktionen nur wenige Abdrucke erreicht werden.

- Eine Reihe von Teilnehmerinnen wurden darüber hinaus durch ihre Frauenärzte informiert (siehe S. 15).

Deshalb wurden vorwiegend Frauen erreicht, die auch nach der Geburt ihrer Kinder weiterhin im Reitsport geblieben sind und durch Veröffentlichungen in Reiterzeitschriften erreicht wurden. Dabei handelt es sich vor allem um Frauen mit langjähriger Reiterfahrung, eigenen Pferden, Wettkampferfahrung und -erfolgen bzw. mit einem beruflichen Engagement als Trainerinnen, Bereiterinnen, Züchterinnen und einem großen zeitlichen Engagement im Reitsport: 617 der insgesamt 1816 Teilnehmerinnen bezeichneten sich selbst als Berufsreiterinnen oder Leistungssportlerinnen im gehobenen Bereich. Frauen mit einer geringeren Bindung an das Reiten, die in ihrer ersten Schwangerschaft mit dem Reiten aufgehört und es anschließend nicht wieder aufgenommen haben, wurden nicht in wünschenswertem Ausmaß erreicht.

\section{$\underline{\text { 5.1.4 Diskussion Alter, BMI, Sport }}$}

In der Reiterinnenstudie waren weniger Frauen unter 24 und über 35 Jahren vertreten als in der Perinatalstudie. Der mittlere BMI lag bei 22,4 und damit niedriger als bei der Perinatalstudie.

Weiterhin geben $60,7 \%$ an, zusätzlich zum Reiten mindestens eine weitere Sportart zu betreiben. Insgesamt verbringen vor der Schwangerschaft - inklusive Reitsport - 83,8\% der Teilnehmerinnen zwei Stunden und mehr pro Woche beim Sport. 51,5\% betreiben auch während der Schwangerschaft mindestens eine weitere Sportart, 56,8\% der Befragten inklusive sportlichen Reitens - berichten 2 Stunden Sport und mehr pro Woche, 32,1\% berichten 4 Stunden Sport und mehr pro Woche bis hin zu mehreren Stunden sportlicher Belastung pro Tag. 
Tab. 15 zeigt die Ergebnisse eines Bevölkerungssurveys des Robert-Koch-Instituts aus dem Jahr 2002/2003 (Gesundheitsberichtserstattung des Bundes 2010). Danach treiben 71,7\% der Frauen im Alter von 18 bis 20 Jahren und 62,9\% der Frauen im Alter von 30 bis 39 Jahren Sport. 42,4\% der Frauen zwischen 18 und 29 Jahren geben an, wöchentlich 4h und mehr zu trainieren, ebenso $32,8 \%$ der Frauen zwischen 30 und 39 Jahren.

Tab. 15. Anteil der Bevölkerung mit sportlicher Betätigung in den letzten 3 Monaten vor der Telefonbefragung nach Umfang/Woche, Alter und Geschlecht, 2002/2003.

\begin{tabular}{|l|c|c|c|c|c|c|c|}
\hline & $\mathbf{n}$ & Kein Sport & $\begin{array}{l}\text { Sport } \\
\text { insgesamt }\end{array}$ & Bis 1 h & Bis 2 h & Bis 4 h & Über 4 h \\
\hline Gesamt & 4.288 & $38,4 \%$ & $61,6 \%$ & $11,9 \%$ & $16,5 \%$ & $17,4 \%$ & $15,8 \%$ \\
\hline $\begin{array}{l}\mathbf{1 8} \text { bis 29 } \\
\text { Jahre }\end{array}$ & 690 & $28,3 \%$ & $71,7 \%$ & $10,2 \%$ & $19,0 \%$ & $23,9 \%$ & $18,5 \%$ \\
\hline $\begin{array}{l}\mathbf{3 0} \text { bis 39 } \\
\text { Jahre }\end{array}$ & 822 & $37,1 \%$ & $62,9 \%$ & $11,1 \%$ & $18,9 \%$ & $17,9 \%$ & $14,9 \%$ \\
\hline $\begin{array}{l}\mathbf{4 0} \text { bis } \mathbf{4 9} \\
\text { Jahre }\end{array}$ & 755 & $36,1 \%$ & $63,9 \%$ & $11,6 \%$ & $18,1 \%$ & $19,5 \%$ & $14,7 \%$ \\
\hline $\begin{array}{l}\mathbf{5 0} \text { bis } \mathbf{6 4} \\
\text { Jahre }\end{array}$ & 992 & $34,2 \%$ & $65,9 \%$ & $12,8 \%$ & $17,4 \%$ & $17,9 \%$ & $17,7 \%$ \\
\hline $\begin{array}{l}\text { ab } \mathbf{6 5} \\
\text { Jahre }\end{array}$ & 1.030 & $52,1 \%$ & $47,9 \%$ & $13,0 \%$ & $10,8 \%$ & $10,4 \%$ & $13,7 \%$ \\
\hline
\end{tabular}

Im Vergleich der schwangeren Reiterinnen mit einer gleich alten, weiblichen, nicht reitenden Normalpopulation zeigt sich, dass die Reiterinnen deutlich mehr Zeit für ihr sportliches Engagement aufbringen: Zu dem Reiten in der Schwangerschaft kommen hier vielfach noch weitere Sportarten hinzu.

Die Frage nach dem Einfluss dieser hohen sportlichen Aktivität auf die Schwangerschaft wurde in der Arbeit nicht untersucht.

\subsection{Frühgeburtlichkeit und Sport}

Alle landläufigen Bestrebungen in der Beratung und Betreuung von Schwangeren münden in das Ziel, die Gesundheit von Mutter und Kind zu gewährleisten und eine Entbindung am Termin zu erreichen. Neben der adäquaten Behandlung mütterlicher Risiken und Erkrankungen und der frühzeitigen Diagnose von Schwangerschaftskomplikationen gehören Fragen des beruflichen und sozialen Umfeldes ebenso wie Ernährung, Sport und Erholung in diesen Komplex (Bahr 2006, Huch 2008). Die Bedeutung eines protektiven psychosozialen Umfeldes (Dudenhausen 2003) und einer optimalen Ernährung (Briese 2001, Friese 2003) sind bekannt. Auch der Einfluss von aufsteigenden Infektionen auf vorzeitige Wehentätigkeit, vorzeitigen Blasensprung und Frühgeburtlichkeit (Saling 2000) wird zunehmend anerkannt. 
Blutungen in der Frühschwangerschaft sind ein zusätzlicher Marker für vorzeitige Geburtsbestrebungen im dritten Trimester (Hossain 2007).

Während Erwerbstätigkeit grundsätzlich kein Schwangerschaftsrisiko darstellt (siehe Kapitel 5.2), sind Maßnahmen zur körperlichen Entlastung werdender Mütter Bestandteil der Gesetzgebung (Gesetz zum Schutz der erwerbstätigen Mutter 1952 und 2009).

Zahlreiche Publikationen beschäftigen sich seit etwa zwei Dekaden mit dem Einfluss sportlicher Betätigung auf Schwangerschaft und Geburt.

Dass auf sportliche Aktivitäten bei Risikoschwangerschaften und Komplikationen verzichtet werden soll, darüber besteht Konsens (ACOG 2002, Paringer 2010). Während jedoch bis in die 1990er-Jahre von Sport in der Schwangerschaft - bis auf geringes Training im WellnessBereich - eher abgeraten wurde (ACOG 1994), mehren sich seitdem die Hinweise darauf, dass sportliche Aktivität für Mutter und Kind nicht nur unschädlich, sondern sogar vorteilhaft sein kann (Bung 1988 und 1999, Huch 1990 und 1996, Hatch 1993, Erhard 2007, Heegard 2008, Schlüssel 2008).

Sport während der Schwangerschaft verbessert die Stimmung während und nach der Schwangerschaft (Kleinert 2008, Reimers 2008, Sulprizio 2009), reduziert Depressivität während der Schwangerschaft und post partum (Strøm 2009), reduziert die Schmerzwahrnehmung und verbessert die Schmerzverarbeitung unter der Geburt (Reimers 2008). Sportlerinnen haben kürzere Geburtsverläufe (Paringer 2010).

Hinweise darauf, dass Freizeitsport das Frühgeburtsrisiko erhöhen würde, lassen sich trotz früherer Bedenken und trotz nachweisbarer Hyperthermie und Kreislaufadaptationen beim Leistungstraining von Schwangeren in der Literatur nicht finden (Hammer 2000, ACOG 2002, Hegaard 2008, Melzer 2010). Körperliche Belastung - Fahrradergometer, Laufband - führen im Langzeit-Wehenmonitoring nicht zu einer Zunahme der Wehentätigkeit (Oxynos 2003).

Weit weniger Literatur findet sich zu der Frage, ob auch Leistungssport mit einem hohen wöchentlichen Trainingspensum in der Schwangerschaft fortgeführt werden kann. Dies ist bis weit in die Schwangerschaft ohne nachteilige Effekte möglich, wie zwei aktuelle skandinavische Studien zeigen (Kardel 2005, Pettinen 2007).

Das wesentliche Augenmerk geht bei Sportlerinnen dahin, die sportliche Betätigung und Wettkampfambitionen zwar fortzuführen, aber individuell angepasst dann zu reduzieren, 
wenn der Verlauf der Schwangerschaft dies erfordert. Diese Entscheidung muss die Sportlerin gemeinsam mit Trainern, Partner, Arzt und Hebamme treffen. Dem sportlichen Ehrgeiz und der Freude am Sport stehen die eigene Gesundheit und die des Kindes gegenüber.

Das Reiten in der Schwangerschaft birgt dem gegenüber einige Eigenheiten. Kasuistische Berichte zu schwangeren Reiterinnen gibt es seit langem. Sie betreffen vor allem professionelle Reiterinnen (Engelhardt 1965, Düntzer 1967, Rogers 2007, Reiten und Zucht 2010). Während allerdings in den gängigen Sportarten die Leistungssteigerung bzw. der Leistungserhalt im Fokus stehen, bietet das Reiten in der Schwangerschaft die Möglichkeit, hohe Leistungen zu erbringen, ohne die Grenzen der körperlichen Kapazitäten zu tangieren. Denn beim Reiten ist - wenn die Anfängerzeiten überwunden sind, die sportliche Grundlage (Heipertz-Hengst 2001, Abt 2005) für ein erfolgreiches Reiten gelegt und ein Steady state des reiterlichen Einsatzes erreicht ist - nicht mehr eine ständige Steigerung der Fitness und körperlichen Leistungsfähigkeit der Reiterin das Ziel. Eine gute körperliche Leistungsfähigkeit und hohes reiterliches Können vorausgesetzt, geht es in diesem Stadium vielmehr um eine Verfeinerung der Kommunikation mit dem Pferd und um das Erreichen anspruchsvollerer Ausbildungsziele, bei denen eine übermäßige Kreislaufbeanspruchung, Hyperthermie, Exsikkose, Laktazidose, Hypoxie und andere Effekte von Trainingsüberlastung keine Rolle spielen. Im Gegenteil, beim Reiten ist es nicht erwünscht, die körperlichen Leistungsreserven anzugreifen, denn der Reiter muss auch in unvorhergesehenen Auseinandersetzungen mit seinem Pferd immer reaktionsfähig bleiben.

Solange die schwangere Reiterin sich wohl, sicher und gesund fühlt, die regelmäßigen Mutterschafts-Vorsorgetermine zur Früherkennung von Schwangerschaftsrisiken wahrnimmt und Unfallgefahren beseitigt, kann also das Reiten - gleich welcher Disziplin und Trainingsintensität - zumindest vom sportlichen Aspekt aus während der Schwangerschaft beliebig fortgeführt werden. Dies beweisen die Daten aus der hier vorgelegten Untersuchung, denn eine Beeinträchtigung des Schwangerschaftsverlaufes und des kindlichen Outcome konnte in der Befragung bei unauffälligen Schwangerschaften auch bei ambitioniertem Reiten mit hoher Trainingsbelastung nicht nachgewiesen werden.

Dabei ist zu beachten, dass die Teilnehmerinnen zahlreiche Maßnahmen zur Adaptation ergreifen und im Durchschnitt in Woche 30 mit dem Reiten aufgehört haben. Teilnehmerinnen, die vorzeitig entbunden haben, haben im Durchschnitt 5,2 Wochen früher mit dem Reiten aufgehört als Schwangere, die in Terminnähe entbunden haben. Dies darf nicht dahingehend interpretiert werden, dass langes Reiten in der Schwangerschaft einer 
Frühgeburtlichkeit vorbeugen könnte. Stattdessen bestand bei diesen Frauen häufiger bereits früh in der Schwangerschaft ein medizinisch begründeter Anlass, die körperliche Belastung zu reduzieren: Die bekannten, mit Frühgeburtlichkeit assoziierten Risikofaktoren fanden sich hier gehäuft bereits während der reiterlichen Aktivität, verstärkt aber in der Zeit nach Beendigung des Sports: Bluthochdruck, vorzeitige Wehentätigkeit, vorzeitiger Blasensprung, Zervixinsuffizienz, Blutungen. Diese Symptomliste ist eng mit dem Risiko einer vorzeitigen Entbindung assoziiert.

Ein wesentlicher psychosozialer Faktor sei zusätzlich erwähnt: Schwangere mit Frühgeburt fühlen sich viel häufiger durch Arbeit, Beruf und Familie belastet als Schwangere mit Entbindung am Termin. Ob diese hohen Stressfaktoren zunächst dazu geführt haben, dass das Reiten aufgegeben wurde, und dann später zur Frühgeburtlichkeit beigetragen haben, sei dahingestellt.

Das Reiten als Sportart stellt zusammenfassend für eine intakte Schwangerschaft kein Risiko dar. Die üblichen Vorsichtsmaßnahmen dürfen nicht außer Acht gelassen werden. Die Entscheidung, die reiterliche Belastung zu reduzieren oder mit dem Reiten aus Rücksicht auf die Schwangerschaft aufzuhören, muss für jede Reiterin auch in einem professionellen Umfeld gewährleistet bleiben.

\subsection{Körperliche Belastung und körperliche Arbeit während der Schwangerschaft}

Reiten und der Umgang mit dem Pferd müssen nicht nur als „Sport“ oder „Freitzeitsport“ betrachtet werden, sondern auch unter dem Aspekt körperlicher Arbeit und der Frage, ob diese Belastung das Frühgeburtsrisiko erhöhen kann. Jede Arbeit am Pferd und die Stallarbeit ist mit körperlicher Belastung verbunden, mit dem Heben von Lasten (Sattelzeug, Futtersäcke, Ausmisten und Einstreuen der Boxen etc.). Ebenso ist das Reiten von mehreren Pferden pro Tag bei Berufsreiterinnen unter dem Aspekt der körperlichen Arbeit zu betrachten.

Ein Einfluss schwerer körperlicher Arbeit auf die Schwangerschaft und Frühgeburtenrate wird kontrovers diskutiert. Einige Untersucher finden eine konstant positive Korrelation zwischen der Dauer und der Intensität körperlicher Arbeit (Luke 1995, Lawson 2009) und Frühgeburtlichkeit: Je länger die wöchentliche Arbeitszeit und je höher die Gesamtmenge der Gewichte, die pro Woche zu heben waren, umso höher das Frühgeburtsrisiko. Teilzeitarbeit, vor allem in ersten Trimester, und das Fehlen von Nachtarbeit waren in der 
Nurses Health Study II bei der Subpopulation von Krankenschwestern mit Schwangerschaft $(n=11.177)$ der wichtigste Parameter, um das Frühgeburtsrisiko zu reduzieren.

Eine Untersuchung an 6674 Erstgebärenden im US-amerikanischen Militär (Ramirez 1990) zeigte, dass hohe und sehr hohe körperliche Anforderungen im Militärdienst zu einer erhöhten Frühgeburtenrate führten (10,1\% vs. $6,1 \%$ bei niedriger Belastung). Die Autoren verwiesen in ihrer Zusammenfassung auf ähnliche Ergebnisse anderer Untersuchungen beim Militär und empfahlen Militärärzten und Vorgesetzen dringend, die einschlägigen Regelwerke zum Schutz werdender Mütter zu beachten.

Andere Untersucher im nicht-militärischen Bereich, vor allem in prospektiven Studien, finden dagegen eine nicht-lineare und kontroverse Beziehung zwischen körperlicher Belastung und Frühgeburtlichkeit (Ahlborg 1990, Croteau 2007, Pompeji 2005). In diesen drei Studien mit insgesamt 10.569 Teilnehmerinnen gab es zwei unterschiedliche Gruppen: Eine Gruppe mit Frauen, die schwere körperliche Arbeit, lange wöchentliche Arbeitszeiten, Heben und Tragen von Lasten ohne Beeinträchtigung ihrer Schwangerschaft leisten konnten, und eine zweite Gruppe, die insgesamt weniger belastungsfähig war, höhere Stressfaktoren aufwies und früher in der Schwangerschaft Arbeitszeit und körperliche Leistung reduzieren musste. In allen drei genannten, großen Untersuchungen war dieser zweiten, leistungsreduzierten Gruppe die Frühgeburtenrate höher als in den Vergleichsgruppen, in denen die Schwangeren höhere körperliche Belastungen über einen längeren Zeitraum während der Schwangerschaft bewältigen konnten.

Die Fragestellungen und Zielsetzungen in den drei genannten Studien waren unterschiedlich, und die Autoren wiesen in ihren Zusammenfassungen auf die Bedeutung der Vorsorgeuntersuchungen, der Mutterschaftsrichtlinien und auf die Notwendigkeit psychosozialer Unterstützung für Schwangere hin.

Trotzdem zeigt sich, dass die Frage nach der körperlichen Belastbarkeit von Schwangeren und ihrem Einfluss auf das Frühgeburtsrisiko nicht linear beantwortet werden kann:

- Physisch und psychisch stabile Schwangere tolerieren körperliche Belastungen ohne erhöhtes Frühgeburtsrisiko,

- Schwangere mit eingeschränkten gesundheitlichen Ressourcen setzen frühzeitig ihren körperlichen Einsatz herab und haben ein erhöhtes Frühgeburtsrisiko. 
Die Ergebnisse der hier zitierten Untersuchungen bestätigen die Resultate der vorgelegten Reiterinnenstudie:

Reiterinnen mit vorzeitiger Entbindung (bis 33. Woche) hören im Durchschnitt 5,5 Wochen früher mit dem Reiten auf als Reiterinnen mit termingerechter Entbindung. Nur 7,1\% der Frauen mit Frühgeburt reiten täglich (16,6\% der Frauen mit Entbindung am Termin). Dies lässt den Schluss zu, dass Reiterinnen mit Risikoschwangerschaft ihre sportliche Aktivität an ihren Gesundheitszustand anpassen, um negative Folgen für sich, die Schwangerschaft und das Kind zu vermeiden.

Bei Reiterinnen ohne gesundheitliches Risiko stellt offensichtlich ein der Situation angepasstes, auch intensives Reiten und die dazu gehörende körperliche Belastung im Umgang mit Pferden kein Risiko für die Schwangerschaft dar.

\section{$\underline{\text { 5.3.1 Berufsreiterinnen }}$}

Wenn eine Berufsreiterin berichtet, dass sie ihre intensive reiterliche Tätigkeit als belastend empfindet, so müssen in die Beratung zusätzliche Gesichtspunkte einfließen. Denn zum Berufsbild der Bereiterin und Reitlehrerin gehören lange tägliche Arbeitszeiten, stundenlanges Stehen beim Reitunterricht, beim Longieren und bei der Pferdepflege, Stallarbeit, das Tragen von Lasten (Sättel, Putzzeug, Heuballen, Futtersäcke). Hier sollte nicht nur eine Reduzierung oder Unterbrechung des Reitens empfohlen werden, sondern die Schwangere sollte auch Tätigkeiten im Stehen vermeiden, weil aus der Arbeitsmedizin bekannt ist, dass das Stehen einen eigenständigen Risikofaktor für die Erhöhung des Frühgeburtsrisikos darstellt.

\subsection{Biometrische Belastungen durch das Reiten}

Die biometrischen Belastungen beim Reiten werden durch mehrere Faktoren beeinflusst:

- Anatomie, Bemuskelung, Schwung, das "Gangwerk“ des Pferdes (Hübener 2004, Witte 2009),

- Form und Lage des Sattels (Witte 2009),

- das Können des Reiters,

- die reiterliche Disziplin und die Reitweise. 
Dass diese Belastungen gesundheitsrelevant werden können zeigt die Tatsache, dass hartnäckige Rückenschmerzen bei Berufsreitern die häufigste Ursache für Anträge auf Anerkennung einer Berufskrankheit sind (Berufsgenossenschaftliches Insitut für Arbeitssicherheit 1999). Rückenschmerzen ohne anatomische Korrelat sind vor allem bei Intensivreitern verbreitet (Hördegen 1975, Heipertz 2001, Kraft 2007). Die erheblichen Schwingungsbelastungen beim Reiten können bei langjähriger Exposition zur Schädigung der Wirbelsäule führen, vor allem dann, wenn bereits latente Vorschädigungen mitgebracht werden und keine Ausgleichsgymnastik betrieben wird (Heipertz-Hengst 2000).

In einer Untersuchung an 488 Elitereitern und 20 Voltigierern (Kraft 2007) zeigte sich, dass 72,8\% der Reiter mit einem Durchschnittsalter von 33,5 Jahren Rückenschmerzen in der Anamnese und zum Untersuchungszeitpunkt aufwiesen $(p<0,05)$. Ein Unterschied zwischen Dressur- und Springreitern in der Häufigkeit der Rückenschmerzen ließ sich nicht finden. In einer Folgestudie desselben Autors fand sich unter den Reitern - und hier vor allem bei den Dressurreitern - eine hohe Zahl an pathologischen Befunden im Magnetresonanztomogramm an den Bandscheiben der Lendenwirbelsäule, auch wenn die Differenz zur Kontrollgruppe wegen der kleinen Fallzahl nicht signifikant war (Kraft 2007). Die Ergebnisse lassen den Schluss zu, dass das Reiten langfristig auch bei sehr gut trainierten Reitern zu Gesundheitsschäden führen kann (bei Reitanfängern wurden die biometrischen Belastungen für Rumpf und Wirbelsäule bisher nicht in objektiv messbaren Szenarien überprüft).

Die biometrischen Belastungen im tiefen Dressursitz mit perfekter Anpassung an das Gangwerk des Pferdes, beim Leichttraben und beim leichten Sitz im Galopp und bei mangelnder Sitzkontrolle sind den Abbildungen 13 und $14 \mathrm{zu}$ entnehmen. Hierbei werden nur die vertikalen Bewegungen des Pferderückens und des Beckenkamms des Reiters abgebildet, weil diese am meisten $\mathrm{zu}$ den biomechanischen Belastungen im Rumpf beitragen. Die zusätzlichen und erheblichen lateralen und horizontalen Amplituden ebenso wie die ständig wechselnde Beschleunigung, deren Kontrolle wesentlicher Bestandteil der reiterlichen Grundausbildung ist, werden an dieser Stelle aus Gründen der besseren Übersichtlichkeit weggelassen. 
Abb. 13. Trab (schematische Darstellung nach Hübener 2004).

Vertikale Bewegungen des Pferdes schwarz, Bewegungen des Reiters grau.

Oben: Geübter Dressursitz.

Mitte: Leichttraben (ein Tritt wird im Sattel gesessen, der zweite im Stehen abgefedert, siehe S. 8-9).

Unten: Ungeübter Sitz mit hoher Belastung für Reiter und Pferderücken.

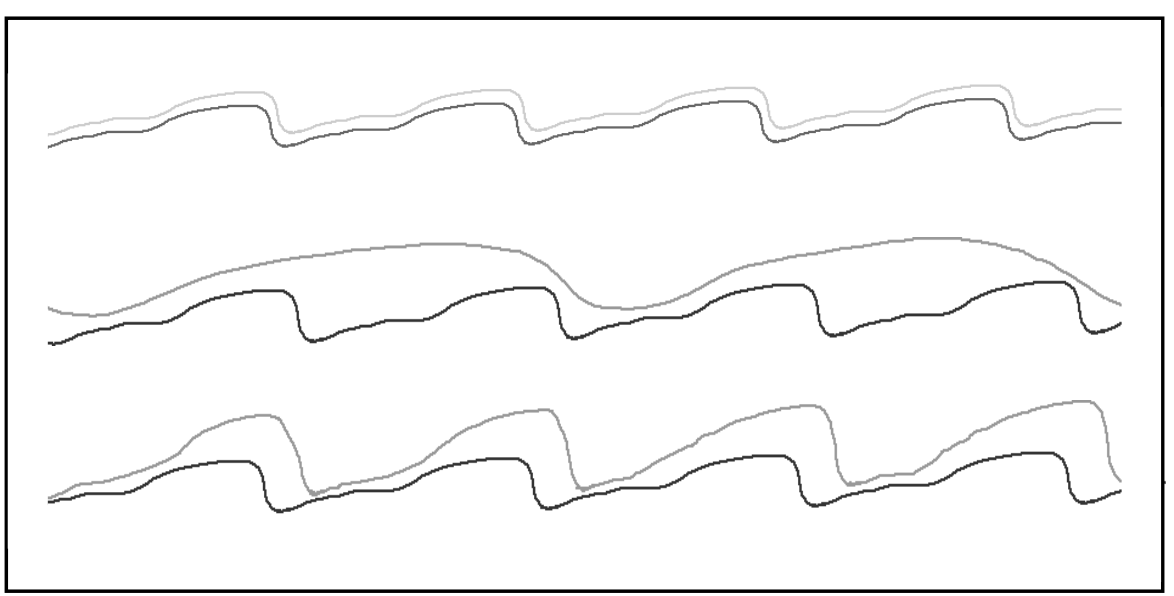

Abb. 14. Galopp (schematische Darstellung nach Hübener 2004).

Vertikale Bewegungen des Pferdes schwarz, Bewegungen des Reiters grau.

Oben: Geübter Dressursitz.

Mitte: Leichter Sitz (Reiter steht ständig in den Steigbügeln).

Unten: Ungeübter Sitz mit hoher Belastung für Reiter und Pferderücken.

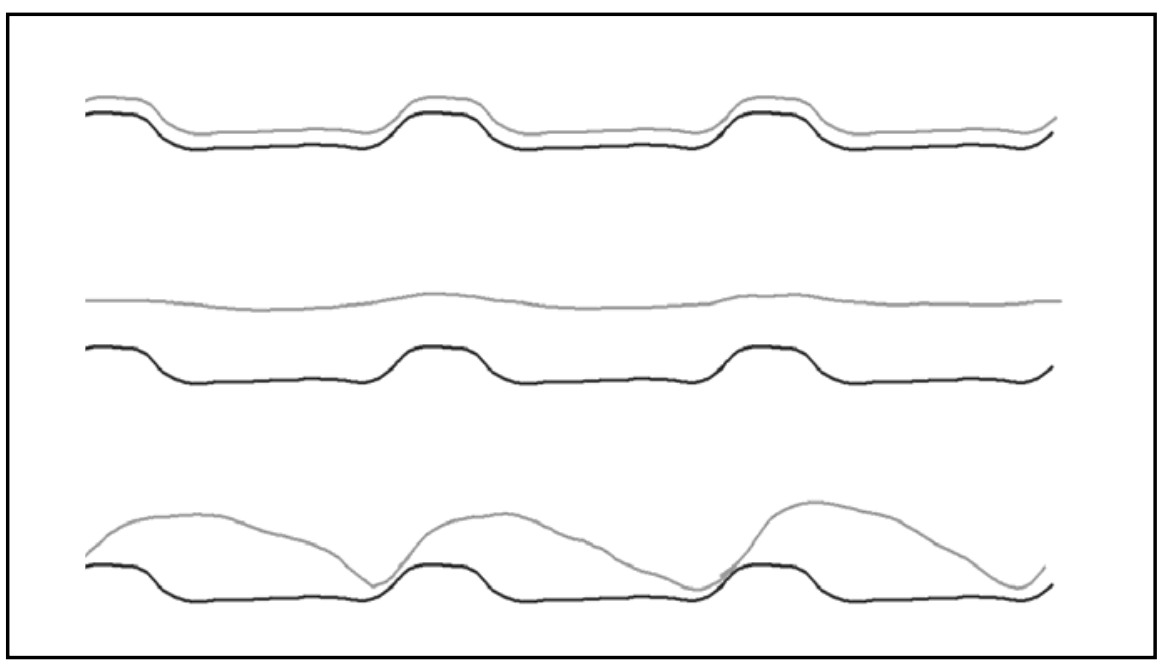

Inwieweit diese Erkenntnisse für das Reiten in der Schwangerschaft angewendet werden können, ist hypothetisch. Denn die biometrische Belastung bei erfahrenen Reitern ist als gleichmäßig und weitgehend physiologisch zu betrachten. Die häufigen Rückenschmerzen bei Viel-Reitern werden eher der Abneigung von Reitern gegen Ausgleichssport 
zugeschrieben als dem Sport selbst (Heipertz-Hengst 2001, Kraft 2007). Auch das stundenlange Stehen beim Reitunterricht und beim Longieren, Mithilfe beim Schmied und Tierarzt mit anstrengender Haltearbeit und alle anfallenden Arbeiten auf dem Reiterhof (Bewegen von Futtersäcken, Hindernissen und anderen Bauelementen des reiterlichen Umfeldes, Heu-, Stroh- und Silageballen, Ausmisten und Einstreu der Boxen etc.) müssen als potentielle Verursacher von Rückenschmerzen in das Gesamtbild mit einbezogen werden. Der mögliche Einfluss dieser Faktoren auf die Schwangerschaft von Reiterinnen wird unter 5.3 diskutiert.

Für die Frage, inwieweit das Reiten als Sport aufgrund seiner biometrischen Belastungen Einfluss auf eine Schwangerschaft haben kann, bleibt festzuhalten, dass Reiten im Grundsatz eine physiologische sportliche Belastung darstellt ohne die im Leistungssport diskutierten Risiken wie Hyperthermie, Exsikkose, plazentarer Hypoxie etc. Es finden sich keine Hinweise darauf, dass das Reiten - auch Spring- und Dressurreiten - einen negativen Einfluss auf eine gesunde Schwangerschaft haben kann. Der biokinetische Einfluss auf den Uterus und den Halteapparat kann reduziert werden, indem der tiefe Dressursitz gegebenenfalls zugunsten des Leichttrabens bzw. des leichten Sitzes aufgegeben wird. In der vorliegenden Untersuchung konnte ein negativer Einfluss des Reitens im Dressursitz oder auch des Springens von Hindernissen auf den Verlauf der Schwangerschaft nicht festgestellt werden.

Fast alle Reiterinnen haben angegeben, dass sie ihren Reitstil im Verlauf der Schwangerschaft angepasst haben - weniger Reitzeit, weniger risikoreiches Reiten, weniger Turnierteilnahmen, mehr Leichttraben, mehr leichter Sitz etc. Diese Entscheidungen ebenso wie die Entscheidung darüber, wann der richtige Zeitpunkt ist, mit dem Reiten aufzuhören, waren im Einzelfall zwar subjektiv und beruhten auf den Ergebnissen der Schwangerenvorsorge, auf der Selbstwahrnehmung der Schwangeren ebenso wie aus der Kommunikation mit Partner, Trainern und anderen Personen des individuellen Umkreises. Aber so subjektiv diese Entscheidungen auch im Einzelfall gewesen sein mögen, so haben sie offensichtlich zu dem Resultat beigetragen, dass das Reiten sich in der vorliegenden Arbeit als eine Sportart gezeigt hat, die keine negativen Einflüsse auf die Schwangerschaft hat.

\subsection{Reiten und Abortrisiko}

Die vorliegende Befragung von Reiterinnen nach Beendigung ihrer Schwangerschaft zeigt mit 16 Aborten und Fehlgeburten (Entbindung vor Ende der 24. Woche) bei 1851 
Teilnehmerinnen eine Abort- und Fehlgeburtenrate von 0,9\%. Diese Rate liegt deutlich unter der nach der Literatur (Baltzer 2006) zu erwartenden Abortrate von 10-15\% ab Konzeption und auch unter der Abort- und Fehlgeburtenrate von 3,6\% ab Bekanntwerden der Schwangerschaft (Madson 2007). Deshalb von einem herabgesetzten Abortrisiko bei Reiterinnen auszugehen, ist nicht angebracht: Da es sich in der vorliegenden Studie nicht um eine repräsentative Stichprobe oder eine prospektive Studie gehandelt hat, hat dieser Wert nur eine beschränkte Aussagekraft. Zumindest findet sich in der Studie keinen Hinweis darauf, dass das Abortrisiko bei Reiterinnen erhöht sein könnte.

$\mathrm{Zu}$ einem anderen Ergebnis kommt eine Subanalyse der Danish National Birth Cohort (Madson et al, 2007). Hier wurde nach einem Zusammenhang zwischen Freizeitsport und Aborthäufigkeit gefragt. In dieser prospektiven, schwangerschaftsbbegleitenden Befragung wurden 92.721 Schwangere eingeschlossen. Die Abortrate bis zur abgeschlossenen 19. Schwangerschaftswoche betrug $3,4 \%(n=3177)$. Die Teilnehmerinnen wurden nach den ausgeübten Sportarten und der Trainingszeit pro Woche befragt. Es fand sich innerhalb der ersten 18 Wochen der Schwangerschaft ein positiver Zusammenhang zwischen der Anzahl der wöchentlichen Trainingsstunden und der Aborthäufigkeit. Das höchste Abortrisiko bestand in der Schwangerschaftswoche 11-14 bei Schwangeren mit einer wöchentlichen Trainingszeit von 7 Stunden und darüber. Nach Beendigung der 18. Woche war ein zusammenhang zwischen Aborthäufigkeit und Trainingsdauer nicht mehr nachweisbar.

Die Untersucher teilten die ausgeübten Sportarten in 5 Gruppen ein:

- High-impact-Sportarten (Jogging, Mannschafts-/Ballsportarten, Racket-Sportarten wie Tennis und Sqash),

- Low impact-Sportarten (Aerobic, Tanzen, Walken/Wandern),

- Workout/Fitnesstraining, Studio,

- Radfahren/Reiten als Sportarten (zwischen den beiden Sportarten wurde nicht unterschieden, es gibt keine Subanalysen),

- Schwimmen.

Nicht berücksichtigt wurden Zweit- und Dritt-Sportarten.

In dieser Untersuchung fand sich bei Schwangeren, die Schwimmen als einzige Sportart angaben, ein reduziertes Abortrisiko. Reiten und Fahrradfahren erhöhten die Hazard Ratio leicht bis zur SSW 18. Alle anderen Sportarten erhöhten das Risiko deutlicher (Tab. 16). 
Tab. 16. Hazard Ratio für Abort und Fehlgeburt in Abhängigkeit von der Sportart und der Dauer der Schwangerschaft in der DNBC ( $n=92.621)$. (Madson et al. 2007)

\begin{tabular}{|l|l|l|l|l|l|}
\hline $\begin{array}{l}\text { Bevorzugte } \\
\text { Sportart }\end{array}$ & $\mathbf{N}$ & $\mathbf{1 1}$ Wochen & $\mathbf{1 1 - 1 4}$ Wochen & $\mathbf{1 5 - 1 8}$ Wochen & $\mathbf{1 9 - 2 2}$ Wochen \\
\hline Kein Sport & 319 & 1 & 1 & 1 & 1 \\
\hline High impact & 49 & 3,6 & 4,2 & 2,1 & 1,2 \\
\hline Low impact & 109 & 2,0 & 1,9 & 1,2 & 0,9 \\
\hline Workout & 20 & 2,1 & 1,9 & 2,0 & 2,3 \\
\hline Fahrrad/Reiten & 79 & 1,3 & 1,7 & 1,3 & 0,7 \\
\hline Schwimmen & 25 & 0,8 & 0,8 & 0,7 & 0,9 \\
\hline Anderes & 20 & 1,0 & 0,9 & 0,8 & 1,0 \\
\hline
\end{tabular}

Die Autorinnen stellen allerdings ihre Untersuchungsergebnisse in Frage und diskutieren einen Untersuchungsbias: Übelkeit (Nausea) in der Frühschwangerschaft tritt, so ihre These, bei Schwangerschaften, die mit einem Abort enden, seltener auf als bei Schwangerschaften, die ausgetragen werden (Lehners 2005). Es sei nicht auszuschließen, dass Schwangere mit intakten Schwangerschaften wegen Übelkeit und Schwangerschaftserbrechen ihre sportliche Aktivität häufiger und früher reduziert haben als Schwangere mit intakten Schwangerschaften, und dass umgekehrt Schwangere ohne Schwangerschaftserbrechen ihre sportliche Aktivität unvermindert fortgeführt und dabei das erhöhte Abortrisiko in diese Gruppe importiert haben. Für eine allgemeine Empfehlung hinsichtlich der sportlichen Aktivität in der Frühschwangerschaft hält die Autorengruppe ihre Ergebnisse deshalb trotz der signifikanten Zusammenhänge derzeit noch nicht für geeignet. Eine Folgestudie der Danish National Birth Cohort gemeinsam mit der Norwegischen Mother and Child Cohort Study ist in Planung und soll über diese Fragestellung detailliertere Auskunft geben.

Jedoch zeigte die Studie auch unter Berücksichtigung des von den Autorinnen diskutierten Bias, dass Schwangere, die im ersten Trimester Joggen, Mannschafts- und RacketSportarten und Workout/Fitness-Studio angaben, ein höheres Abortrisiko bestand als beim Reiten und Fahrradfahren und beim Schwimmen ein niedrigeres Risiko.

Die Ergebnisse sind für die Fragestellung, ob das Reiten in der Schwangerschaft das Abortrisiko erhöht, und welche reiterlichen Aktivitäten hier als besonders risikoreich anzusehen sind, bedingt anwendbar.

Wenn man intensives dressurmäßiges Reiten und intensives Springtraining zu den Highimpact-Sportarten mit Workout-Komponente (Kraftausdauer plus biometrische Belastung) zählt, Reiten im leichten Sitz zu den Low- oder Very-low-impact-Sportarten, dann könnte die 
Empfehlung lauten, intensives dressurmäßiges Reiten und forciertes Springen bis zur 18. Woche zu reduzieren, um auf diese Weise das Abortrisiko zu vermindern. Diese Empfehlung ist allerdings hypothetisch.

Reiterinnen betreiben häufig mehrere Sportarten intensiv, wie auch die vorgelegte Untersuchung zeigt. Eine zweite Schlussfolgerung könnte also sein, dass das erhöhte Abortrisiko von Radfahrerinnen und Reiterinnen dadurch vermindert werden kann, dass die Begleitsportarten sorgfältig ausgewählt werden. Sekundär betriebene High-impactSportarten sollten möglicherweise im ersten Trimester durch Schwimmen ersetzt werden, um das erhöhte Abortrisiko zu reduzieren; auch diese Empfehlung ist hypothetisch.

Diese Ergebnisse sollten allerdings vor dem Hintergrund gesehen werden, dass in der hier vorgelegten, retrospektiven Reiterinnenstudie an einem Kollektiv ohne Kontrollgruppe eine Abortrate von 1,2\% gefunden wurde. Selbst wenn die Abort- und Fehlgeburtenrate in der Reiterinnenstudie durch einen Untersuchungsbias falsch niedrig sein sollte, so gibt die Untersuchung keine Hinweise darauf, dass das Reiten in der Schwangerschaft zu einem erhöhten Risiko für Aborte und Fehlgeburten führen kann.

\subsection{Beckenboden und Reiten in der Schwangerschaft}

Empirisch berichten Geburtshelfer und Hebammen, dass Reiterinnen eine festere Beckenbodenmuskulatur haben als Nichtreiterinnen, und dass dies den Geburtsverlauf negativ beeinflussen könne. Da es sich in der vorliegenden Studie um eine retrospektive Befragung an nicht medizinisch vorgebildeten Teilnehmerinnen handelt, und die Datenerhebung sich ausschließlich auf die Angaben der Teilnehmerinnen stützt, kann die Frage nach einer möglicherweise verlängerten Dauer der Einleitungs- bzw. Austreibungsphase nicht beantwortet werden. Stattdessen wurden die Häufigkeiten sekundärer Sectiones und vaginal-operativer Entbindungen als klinische Parameter herangezogen, um eine mögliche Beeinträchtigung des Geburtsverlaufs zu evaluieren.

Zunächst muss gefragt werden, ob Sport und insbesondere Reiten überhaupt einen Einfluss auf die Beckenbodenmuskulatur haben. Diese Frage kann nach der aktuellen Literaturlage bejaht werden: Untersuchungen aus jüngster Zeit zeigen, dass Hochleistungssportlerinnen eine erhöhte Muskelmasse und einen erhöhten Grundtonus der Beckenbodenmuskulatur aufweisen (Kruger 2007). Im EMG konnte die Zunahme des Muskeltonus während des Reitens in Abhängigkeit von der Belastung gemessen werden (Schäfer 2009). In einer 
Befragung von Reiterinnen, Schwimmerinnen und Radfahrerinnen wiesen Reiterinnen die niedrigste Rate an Harninkontinenz-Symptomen auf; die Autoren werteten dies als Zeichen dafür, dass das Reiten die Beckenbodenmuskulatur trainiert (Alanee 2009).

In der Auswertung der Reiterinnenstudie zeigte sich insgesamt bei getrennter Auswertung der Primi- und Multiparae im Vergleich zur Niedersächsischen Perinatalstudie keine erhöhte Rate an vaginal-operativen Entbindungen, während die Zahl der primären Sectiones signifikant erniedrigt war. Allerdings konnte kein Zusammenhang zwischen der Häufigkeit vaginal-operativer Entbindungen und sekundärer Kaiserschnitte und der Intensität und Dauer der reiterlichen Aktivität gefunden werden: Vaginal-operative Geburtsbeendigungen und ungeplante Kaiserschnitte waren bei Frauen, die früh in der Schwangerschaft mit dem Reiten aufgehört haben und solchen, die bis in Terminnähe weitergeritten sind, gleich häufig, ebenso bei Frauen, die in der Schwangerschaft wenig $-2 \mathrm{~h}$ pro Woche und darunter - oder viel - $14 \mathrm{~h}$ pro Woche oder mehr - geritten sind. Die einzige Schlussfolgerung, die aufgrund dieser Daten möglich ist, ist die, dass das jahrelange reiterliche Training möglicherweise bereits vor der Schwangerschaft den Beckenboden so trainiert, dass der Trainingseffekt auch während der Schwangerschaft erhalten bleibt, unabhängig von der Intensität des Reitens während der Schwangerschaft selbst.

Neben der postulierten, hier aber nicht nachweisbaren Beeinträchtigung des Geburtsverlauf durch den Reitsport gibt es in der Literatur Hinweise darauf, dass eine gut trainierte Beckenbodenmuskulatur zahlreiche gesundheitliche Vorteile hat. Sie führt zu einer geringeren Rate an Dammrissen, und sie reduziert die Folgesymptomatik nach einer Episiotomie (Klein 1997). Beckenbodentraining während der Schwangerschaft reduziert zudem das Inkontinenzrisiko während und nach der Schwangerschaft (Kölbl 2003).

Die Hypothese, dass eine durch das Reiten gut trainierte Beckenbodenmuskulatur den Geburtsverlauf klinisch relevant und negativ beeinflussen kann, kann durch die in der Reiterinnenstudie erhobenen Daten nicht bestätigt werden. 


\subsection{Beckenendlage und Reiten in der Schwangerschaft}

In der Literatur werden folgende Risiken für Entbindung aus Beckenendlage genannt:

- Niedriges Geburtsgewicht,

- Frühgeburt,

- Hydrozephalus,

- niedriger Plazentasitz,

- kurze Nabelschnur,

- Rauchen,

- Diabetes,

- erhöhte Gewichtszunahme während der Schwangerschaft (Rayl 2005, Talas 2009).

Ein Zusammenhang zwischen physischer Belastung und Beckenendlage wurde bisher nicht untersucht.

Deshalb wurde im Rahmen der hier vorgelegten Studie zum Reiten in der Schwangerschaft die Kindslage zum Zeitpunkt der Entbindung mit den reiterlichen Aktivitäten in Zusammenhang gebracht.

Die Hypothese, dass das Reiten in der Schwangerschaft generell zu einer Zunahme von Beckenendlagen-Geburten führt, kann mit den vorliegenden Daten für ungültig erklärt werden. Die Subgruppen-Analyse zeigt, dass das dressurmäßige Reiten vor und während der Schwangerschaft das Risiko für eine Beckenendlage geringfügig ansteigen lässt. Dieses Risiko ist unabhängig davon, wie lange das Reiten während der Schwangerschaft fortgeführt wird.

Die geringfügige Neigung des Feten, sich bei dressurmäßigem Reiten der Mutter in Beckenendlage einzustellen, ist aus reiterlicher Sicht zumindest hypothetisch zu erklären, denn die biometrischen Belastungen sind beim tiefen Dressursitz höher als beim Reiten im leichten Sitz (siehe S. 8-9).

Diese unterschiedlichen biomechanischen Belastungen könnten der Grund dafür sein, warum sich Feten in Schwangerschaften mit dressurbetontem Reiten etwas häufiger in Beckenendlage einstellen als in Schwangerschaften, in denen die Reiterinnen auf den betont tiefen Sitz verzichten. Beweisen lässt sich diese Annahme nicht. 
Folgen der unterschiedlichen Reitweise für die Dauer der Schwangerschaft oder die Gesundheit des Feten konnten nicht gefunden werden - außer einem hohen Anteil an Kaiserschnitten zur Entbindung aus Beckenendlage.

\subsection{Unfälle durch Reiten und Umgang mit dem Pferd in der Schwangerschaft}

\section{$\underline{\text { 5.8.1 Unfälle in der Schwangerschaft }}$}

Zu Reitunfällen in der Schwangerschaft liegen keine Publikationen vor.

6-7\% aller Schwangeren in den USA erleiden während der Gravidität irgendeine Form von Trauma; etwa 10\% davon erfordern eine Hospitalisierung (Chang 2009).

In einer umfangreichen, bevölkerungsbasierten Statistik aus den USA zu Unfällen in der Schwangerschaft in den Jahren 1991 bis 1999 (El Kady 2004) wurde gezeigt, dass bei 4.833.286 einbezogenen Entbindungen 10.316 (0,2\%) im Zusammenhang mit einem hospitalisierungspflichtigen Trauma stattfanden. Davon mündeten 2.494 in eine vorzeitige Entbindung kurz nach dem Trauma $(24,1 \%)$. Die Rate an Fehl- bzw. Totgeburten war mit einer OR von 4,4 deutlich erhöht.

Die Überlebenschancen für den Feten waren aber, eine korrekte Versorgung der Mutter vorausgesetzt - weniger von der Schwere des Unfalls abhängig als vielmehr vom Zeitpunkt des Unfalls, der Geburtsbestrebungen und der Entbindung.

Grundsätzlich bedeuten Traumen jeder Art in der Schwangerschaft ein Risiko für Mutter und Kind, und sie stellen die häufigste nicht-geburtshilfliche Ursache für mütterliche und kindliche Todesfälle während der Schwangerschaft dar (Aitokallio-Tallberg, 1997, El-Kady 2004, Schroeder 2007). Die häufigste Ursache sind Tötung und Selbsttötung, gefolgt von Verkehrsunfällen (KfZ, Motorrad, Fahrrad) und Drogenabusus (Mahoney 2010). Bei schweren Verkehrsunfällen kommt es in 30-50\% zu Plazentalösungen (El-Kady 2004, Chang 2007), bei leichten Traumen in 1-5\% (Schroeder 2007). Direkte Verletzungen des Fetus und Uterusrupturen sind eine Rarität und treten nur bei gleichzeitigen, schweren Verletzungen der Schwangeren auf (Chang 2007). Uterusrupturen gehen in $100 \%$ mit einem Verlust des Feten einher und haben eine mütterliche Letalität von 10\% (Schroeder 2007). Die häufigste fetale Todesursache ist mütterliche und fetale Hypoxie nach Kreislaufschock und/oder Blutverlust, gefolgt von Infektionen, Drogen- und Medikamenteneinfluss und vorzeitigen 
Wehen nach vollständiger oder partieller Plazentaablösung mit Abort bzw. Fehlgeburt (Mahoney 2010).

In der älteren Literatur wird postuliert, dass Unfälle mit starker Krafteinwirkung und Körpererschütterung in den ersten vier Schwangerschaftsmonaten häufiger zu einer Ausstoßung der Frucht führen als danach, weil in dieser Zeit die Verankerung der Chorionzotten an der Uteruswand noch locker und vulnerabel sei (Seitz 1933). In der aktuellen Literatur lässt sich kein Hinweis darauf finden, dass Unfälle in der ersten Hälfte der Schwangerschaft häufiger zu einem Verlust des Kindes führen als in der zweiten Hälfte. Allerdings schließen mehrere Untersuchungen zum Outcome nach Unfällen in der Schwangerschaft Ereignisse vor Erreichen der 20. Schwangerschaftswoche aus (El-Kady 2004).

\subsubsection{Unfälle beim Reiten}

In den USA werden bei etwa 30 Mio Reitern jährlich etwa $50.000(0,16 \%)$ in einer Notaufnahme ärztlich behandelt (Carillo 2007).

Nach einer repräsentativen Haushaltsbefragung in den Jahren 2000/2001 (Bundesanstalt für Arbeitsschutz und Arbeitsmedizin BauA 2002) ereignen sich in Deutschland jährlich bei Personen über 14 Jahre 1,46 Millionen Unfälle, davon 56.000 beim Reiten oder beim Umgang mit dem Pferd. Hier sind alle Personen eingeschlossen, die Umgang mit Pferden haben, unabhängig vom Geschlecht, ihrem Versicherungsstatus, Vereinszugehörigkeit und ihrer Tätigkeit (Hobby und Beruf, Landwirtschaft, Zucht, Pferdehaltung, Ausbildung von Pferd und Reiter, Tiermedizin, Fahrsport, Reiten im Verein ebenso wie ohne organisiertes Engagement). Bei 1,24 Mio Reitern in Deutschland ab 14 Jahre (Deutsche Reiterliche Vereinigung, 2001) und einer unbekannten Zahl weiterer mit Pferden beschäftigter Personen entspricht dies einer Unfallhäufigkeit von weniger als 4,5\% pro Jahr.

Nach einer Untersuchung der Ruhr-Universität Bochum gemeinsam mit der ARAG (Henke 2002) an im Verein organisierten Sportlerinnen und Sportlern ereignen sich jährlich etwa 11.000 Unfälle bei Reiterinnen über 14 Jahre, die im Vereinssport organisiert sind $(n=547.256)$. Damit hat Reiten, bezogen auf die im Vereinssport organisierten Sportlerinnen (Deutscher Olympischer Sportbund 2003, 2005) ein geringeres Unfallrisiko als Handball und Volleyball (Tab. 17). Dies widerspricht der landläufigen Meinung, Reiten sei der Sport mit dem höchsten Unfallrisiko (Ball 2007). 
Tab. 17. Unfälle im Vereinssport pro Jahr ( $\left({ }^{1}\right.$ Deutscher Olympischer Sportbund 2003, ${ }^{2}$ Henke 2002).

\begin{tabular}{|l|c|c|c|c|l|}
\hline & Handball & Volleyball & Reiten & Fußball & Turnen \\
\hline $\begin{array}{l}\text { Im Vereinssport organisierte Frauen } \\
\text { über 14 Jahre }\end{array}$ & 313.891 & 253.099 & 547.256 & 838.878 & 3.585 .878 \\
\hline $\begin{array}{l}\text { Unfälle bei Frauen über 14 Jahre im } \\
\text { Vereinssport }\end{array}$ & 34.000 & 18.000 & 11.000 & 13.000 & 14.000 \\
\hline$\%$ Unfälle bezogen auf Mitglieder & $10,8 \%$ & $7,1 \%$ & $2,1 \%$ & $1,5 \%$ & $0,39 \%$ \\
\hline
\end{tabular}

Allerdings sind Reitunfälle wegen der Fallhöhe, der Dynamik des Sports vor allem beim Spring- und Geländereiten und wegen der Krafteinwirkung der Tiere oft schwerer als in anderen Sportarten (Dittmer 1991, Ball 2007). Von Verletzungen betroffen sind in absteigender Reihenfolge

- obere Extremität (Arme und Schultergürtel),

- untere Extremität (Beine und Hüftgelenke),

- Kopf und Gesicht (inklusive Gehirnerschütterungen),

- Wirbelsäule;

- Traumen im Brust- und Bauchbereich ereignen sich selten, zählen dann aber häufig zu den schweren Verletzungen (Bixby-Hammett 1990, Dittmer 1991, Ball 2007).

Ein hohes Risiko haben vor allem unerfahrene Reiter auf der einen Seite und die Gruppe erfahrener, ambitionierter, gut trainierter Reiter auf der anderen Seite, die junge oder anspruchsvolle Pferde ausbilden, korrigieren und auf den Wettkampfsport vorbereiten (siehe S. 11-12), wobei in dieser Gruppe schwerere Verletzungen auftreten als bei den Anfängern (Ball 2007, Newton 2005, Bixby Hammett 1990, Rathfelder 1995). Ein besonderes Risiko für Kopf- und Wirbelsäulenverletzungen weisen erfahrene Spring- und Geländereiter mit Wettkampf-Teilnahmen auf (Silver 2002). Erfahrene und professionelle Reiter haben zudem ein hohes Wiederholungsrisiko (Ball 2009, Bixby-Hammett 1990). Alkoholkonsum erhöht wegen der beeinträchtigten Reaktions- und Koordinationsfähigkeit das Risiko, dass Reitunfälle mit gravierenden Verletzungen einhergehen (Carillo 2007).

\section{$\underline{\text { 5.8.3 Unfälle beim Umgang mit dem Pferd }}$}

Verlässliche Zahlen zum Unfallrisiko beim Umgang mit Pferden existieren nicht. Das Risiko für Unfälle im Umgang mit dem Pferd wird von manchen Untersuchern als höher eingeschätzt als das Unfallrisiko beim Reiten (Rathfelder 1995). Beim Bundesverband Landwirtschaftlicher Berufsgenossenschaften, bei denen eine große Zahl an Pferdewirten 
versichert ist, wurden im Jahr 19994451 Unfälle im Umgang mit Pferden gemeldet (Bundesverband Landwirtschaftlicher Berufsgenossenschaften 2004). Allerdings ist die Gesamtzahl der Versicherten und Mitversicherten, die Umgang mit Pferden haben, nicht bekannt und somit eine Einschätzung des Unfallrisikos nicht möglich.

\section{$\underline{\text { 5.8.4 Unfälle in der Reiterinnen-Studie }}$}

In der hier vorgelegten Reiterinnenstudie wurde eine Unfallhäufigkeit von 9,6\% in einem individuellen Zeitraum von 9 Monaten beim Reiten oder beim Umgang mit dem Pferd gefunden. Diese Unfallhäufigkeit liegt erheblich höher, als alle bekannten Statistiken vermuten lassen. Als wesentliche Ursache für diese Differenz kann vermutet werden, dass die Teilnehmerinnen alle Unfälle beim Reiten oder beim Umgang mit dem Pferd berichtet haben, auch solche, die nicht zu einer ärztlichen Behandlung oder einer Meldung bei ihrem Verein geführt haben, während in anderen Studien das wesentliche Einschlusskriterium eine ärztliche Behandlung und/oder Meldung an einen Versicherer war. Zudem könnte es sich um einen Untersuchungsfehler handeln, denn es ist nicht auszuschließen, dass Reiterinnen mit Unfällen in der Schwangerschaft sich stärker an der Studie beteiligt haben als Reiterinnen ohne Unfälle, .

In der Untersuchung wurde festgestellt, dass vor allem sehr erfahrene, professionell reitende Schwangere ein hohes Unfallrisiko aufweisen. Dieses Ergebnis stimmt mit allen gängigen Unfallstatistiken überein. Der Anteil der Studienteilnehmerinnen, die sich selbst als ungeübte Reiterinnen bezeichneten, war mit 2,4\% erwartungsgemäß sehr niedrig. Eine Unfallhäufung in dieser Gruppe konnte nicht festgestellt werden.

Folgende Faktoren erhöhen das Unfallrisiko bei schwangeren Reiterinnen:

- Reiten von und Umgang mit Problem- und Ausbildungspferden,

- Ambitioniertes Springtraining,

- hohe reiterliche Qualifikation (entsprechend Turniererfolgen in der mittleren und schweren Klasse).

Folgende Faktoren können als zusätzliche Marker für ein erhöhtes Unfallrisiko gewertet werden:

- Frühere Unfälle beim Reiten oder im Umgang mit Pferden,

- durchschnittliche tägliche Reitzeit von $2 \mathrm{~h}$ und darüber auch während der Schwangerschaft, 
- Selbsteinschätzung der Schwangeren als unfallgefährdet („Ich ritt mit einem gewissen Risiko"),

- erhöhte Stressbelastung in der Schwangerschaft, die möglicherweise zu einem Nachlassen der erforderlichen Konzentration führt.

Folgt man den Selbstauskünften der Teilnehmerinnen, so spielten Alkohol und andere Drogen, die andere Untersucher bei Unfällen in der Schwangerschaft häufig unfallbegleitend finden (Chang 2007, Mahoney 2010), in der Reiterinnenstudie keine Rolle. Ob diese Selbstauskünfte unvollständig waren, kann nicht überprüft werden.

Bei den 151 Teilnehmerinnen mit Unfällen in der Schwangerschaft traten nur in 15\% vorzeitige Wehen auf. Dies entspricht den Daten der oben vorgestellten Studien zu vorzeitiger Wehentätigkeit nach nicht-reiterlichen Traumen. In einem Fall kam es zum Verlust der Schwangerschaft, in einem weiteren zur Frühgeburt in der 33. Woche. Alle anderen Schwangerschaften konnten - ungeachtet der Traumatisierungen der Schwangeren - bis zum Termin weitergeführt werden.

Die Auswertung zeigt, dass das Unfallrisiko duch sicherheitsbewusstes Reiten vermutlich gesenkt werden kann. Zu den wesentlichen Maßnahmen gehören erhöhte Aufmerksamkeit beim Umgang mit jungen und schwierigen Pferden, Kompromissbereitschaft im Hinblick auf Ausbildungs- und Wettkampfziele, gelassener Leistungserhalt statt Leistungssteigerung beim Springtraining, Stressreduzierung und Einsatz von Stressbewältigung-Maßnahmen im reiterlichen und nicht-reiterlichen Alltag.

Trotzdem gilt für den Umgang mit dem Pferd grundsätzlich „No horse is a safe horse“. Damit besteht für jede Schwangere beim Reiten und beim Umgang mit dem Pferd ein gewisses Unfallrisiko. Dieses Risiko lässt sich auch bei sehr sicherheitsbewusstem Verhalten nicht ausschalten.

Während das Reiten selbst nach den Ergebnissen der Studie keinen negativen Einfluss auf eine intakte Schwangerschaft hat, sollte das Unfallrisiko bei der Fragestellung, ob das Reiten einen schädigenden Einfluss auf die Schwangerschaft haben kann, nicht außer Acht gelassen werden. 


\section{Summary}

Ziele: Das Ziel der vorliegenden Studie war es, den Einfluss des Reitens, unterschiedlicher reiterlicher Disziplinen, der reiterlichen Erfahrung und der Intensität und Dauer des Reitens vor und während der Schwangerschaft auf Fehlgeburtlichkeit, Frühgeburtsbestrebungen und den Geburtsverlauf zu evaluieren.

Material und Methoden: An der retrospektiven, schriftlichen Befragung nahmen 1858 Reiterinnen teil, die vor oder während ihrer Schwangerschaft geritten waren, und deren Schwangerschaft beendet war.

Ergebnisse: Reiten während der Schwangerschaft hatte keinen Einfluss auf die Abortrate, auf die Dauer der Schwangerschaft oder das kindliche Outcome, weder Dressur- oder Springreiten noch eine reiterliche Aktivität von 20 Stunden und mehr pro Woche. Als wichtigste mit Frühgeburtlichkeit assoziierte Faktoren wurden ein niedriger BMI, Stressbelastung in der Schwangerschaft, hoher Blutdruck und andere Erkrankungen der Mutter und Schwangerschaftskomplikationen gefunden.

Schwangere mit Frühgeburten belasteten sich bereits lange vor dem Auftreten vorzeitiger Wehen reiterlich geringer als Schwangere mit Entbindung am Termin. Sie hörten im Durchschnitt 5 Wochen früher mit dem Reiten auf, und zwar lange bevor klinische Zeichen von Frühgeburtsbestrebungen auftraten.

Die durchschnittliche Dauer des Weiterreitens während der Schwangerschaft betrug 29,6 Wochen. Die durchschnittliche Dauer der Schwangerschaft bei allen Teilnehmerinnen betrug 39,5 Wochen.

Die Rate an sekundären Kaiserschnitten und an vaginal-operativen Entbindungen war im Vergleich mit den Daten aus der Niedersächsischen Perinatalerhebung nicht erhöht.

In 16 Fällen wurden Aborte berichtet. Dies entspricht 0,9\% der Teilnehmerinnen.

Reitunfälle oder Unfälle im Umgang mit dem Pferd wurden von 9,6\% der Teilnehmerinnen berichtet. Risikofaktoren waren eine hohe Professionalität, der Umgang mit jungen und mit Problempferden, Springreiten mit mittleren und hohen Ambitionen und hohe Stressbelastung. In einem Fall endete ein Sturz vom Pferd mit einer Fehlgeburt, in einem 
anderen Fall in einer Frühgeburt ohne Langzeitschäden beim Kind. In allen anderen Fällen konnte trotz der Traumatisierungen der Entbindungstermin erreicht werden.

Diskussion: Bei einer intakten Schwangerschaft und einer gesunden, belastungsfähigen Reiterin hat das Reiten keinen negativen Einfluss auf den Verlauf der Schwangerschaft, der Entbindung und auf das Frühgeburtsrisiko.

Eine Erhöhung der Abortgefahr durch das Reiten in den ersten Schwangerschaftsmonaten kann nicht festgestellt werden.

Reiterinnen, deren Schwangerschaft mit einer Frühgeburt endet, belasten sich reiterlich geringer und hören deutlich früher mit dem Reiten auf als Teilnehmerinnen mit Entbindung am Termin. Es kann vermutet werden, dass diese Frauen eine eingeschränkte physiologische Reserve und eine eingeschränkte Belastungsfähigkeit in die Schwangerschaft mitbringen, die dann zu vermehrten Komplikationen und zur Frühgeburtlichkeit führt.

Da eine prospektive Einschätzung des Schwangerschaftsverlaufs nur eingeschränkt möglich ist, können eindeutige Entscheidungskriterien, ob und wie lange eine Frau in der Schwangerschaft weiterreiten kann, nicht definiert werden. Es wird sich hierbei zumeist um eine multifaktorielle, der ärztlichen Erfahrung und der Selbsteinschätzung der Frau überlassene Entscheidungsfindung handeln.

Berufsreiterinnen, die ihre reiterlichen Aktivitäten als belastend für ihre Schwangerschaft empfinden, sollten zusätzlich darauf achten, Tätigkeiten im Stehen zu reduzieren.

Die Unfallgefahr ist beträchtlich und kann selbst durch sehr risikobewusstes Verhalten nicht völlig eliminiert werden. Deshalb sollte jede Schwangere - ungeachtet ihres Könnens und ihrer Erfahrung - eigenverantwortlich entscheiden, ob Sie bereit ist, für den Gewinn an reiterlicher Lebensqualität die Gefährdung für sich und ihr Kind in Kauf zu nehmen. 


\section{Literatur}

1. Abt S, Sind Reiter Sportler? Reiter Revue 2005;12;13-16.

2. ACOG Committee opinion No 267. Exercise Druing Pregnancy and the Postpartum Period. Obstetrics and Gynecology 2002;99:171-173.

3. Ahlborg G, Bodin L, Hogstedt C. Heavy Lifting During Pregnancy - A Hazard to the Fetus? Int J Epidemiol 1990;19:90-97.

4. Aitokallio-Tallberg A, Halmesmäki E. Motor vehicle accident during the second or third trimester of pregnancy. Acta Obstet Gynecol Scand 1997;76:313-317.

5. Alanee S, Heiner J, Liu N, Monga M. Horseback riding: Impact on sexual dysfunction and lower urinary tract symptoms in men and women. Urology. 2009;73:109-114.

6. Alfredson G, Hedberg G, Bergstrom E, Nordstrom P, Lorentzon R. High Thigh Muscle Strenght but not Bone Mass in Young Horseback-Riding Females. Calcif Tissue Int 1998:62:497-501.

7. Artal R, O'Toole M. Guidelines of the American College of Obstetricians and Gynecologists for exercise during pregnancy and the postpartum period. Br J Sports Med 2003:37:6-12

8. Bahr K, Thoms K, Elkeles T. Schwangerenvorsorge zwischen Risiko- und Ressourcenorientierung. Frauenarzt 2006;47:904-908.

9. Ball C, Ball J, Kirkpatrick A, Mulloy R. Equestrian injuries: Incidence, injury patterns and risk factors for 10 years of major traumatic injuries. The American Journal of Surgery. 2007;193:636640.

10. Ball JE, Ball C, Molloy R, Datta I, Kirkpatrick AW. Ten years of major equestrian injury: Are we adressing functional outcomes? Journal of trauma management \& outcomes. 2009;3:2.

11. Baltzer J, Friese K, Graf M, Wolff F. Praxis der Gynäkologie und Geburtshilfe. Stuttgart 2006:430.

12. Baumann H, Schneider KTM, Fallenstein F, Huch R, Huch A. Fliegen in der Schwangerschaft: Auswirkungen auf den mütterlichen Organismus. Arch Gynecol Obstet 1985;238:292.

13. Berufsgenossenschaftliches Institut für Arbeitssicherheit (Hrsg.). GanzkörperSchwingungsbelastung beim Reiten von Pferden. In „Aus der Arbeit des BIA“. 1999;12:617.BIA:638.23.

14. Bixby-Hammett D, Brooks WH. Common injuries in horseback riding. A review. Sports Med. 1990 Jan;9:36-47.

15. Bundesanstalt für Arbeitsschutz und Arbeitsmedizin (Hrsg.). Sportunfälle 2000. Dortmund 2002. http://www.baua.de/cln_135/de/Informationen-fuer-die-Praxis/Statistiken/Unfaelle/HeimFreizeit/2000/Sportunfaelle_content.html. 10.4.2010.

16. Bung $P$, Spätling L, Huch R, Huch A. Performance training in pregnancy. Report of respiratory and cardiovascular physiologic changes in a pregnant high-performance athlete in comparison with a sample of normal pregnant patients.

Geburtshilfe und Frauenheilkunde 1988;48:500-11.

17. Bung P. Lifestyle in der Schwangerschaft. Teil 3: Sport, körperliche Aktivität, Sexualität. Frauenarzt 2005;46:280-284.

18. Bung P. Schwangerschaft und Sport. Der Gynäkologe 199;5:386-388.

19. Carillo EH, Varnagy D, Bragg SM, Levy J, Riordan K. Traumatic injuries assicated with horseback riding. Scand J Surg. 2007;96(79-92.

20. Chang A. Pregnancy Trauma. http://emedicine.medscape.com/article/796979-print.pdf. 2009 Feb (Update).

21. Croteau A, Marcoux S, Brisson C. Work Acitivity in Pregnancy, Preventive Measures, and the Risk of Preterm Delivery. Am J Epidemiol 2007;166:951-965.

22. Dawson R. Horse Accidents with Injuries: July through September 2002. http:asci.uvm.edu/equine/law/articles/fall_2002.htm Cited 2010 Apr 10.

23. Deutsche Reiterliche Vereinigung. Faszination Zukunft - Neue Perspektiven im Pferdesport. Marktanalyse Pferdesportler in Deutschland. Warendorf 2001. 
24. Deutsche Reiterliche Vereinigung. Jahresbericht 2004. Warendorf 2005.

25. Deutscher Olympischer Sportbund. Bestandserhebung 2003 der Landessportbünde, der Spitzenverbände und weiterer Mitgliedsorganisationen. Frankfurt/Main, 15. November 2003, ISBN 3-89152-108-1.

26. Deutscher Olympischer Sportbund. Bestandserhebung 2009 der Landessportbünde, der Spitzenverbände und weiterer Mitgliedsorganisationen. Frankfurt/Main, 2. überarbeitete Auflage 15. Januar 2010.

27. Dittmer $\mathrm{H}$. The injury pattern in horseback riding. Langenbecks Arch Chir Suppl Kongressbd. 1991():466-469.

28. Dudenhausen J, Kirschner R. Psychosoziale Belastungen als Risikofaktoren der Frühgeburt Erste Befunde der Daten des BabyCare-Projekts. Zentralbl Gynäkol 2003;125:112-122.

29. Duncombe D, Wertheim EH, Skouteris H, Paxton SJ, Kelly L. Factors relates to exercise over the course of pregnancy including women's beliefs about the safety of exercise during pregnancy. Midwifery 2009;25:430-38.

30. Düntzer E. Die Frau im Sattel. MMW 1967;23:1269-1271.

31. Ebner F. Schwangerschaft und Fallschirmspringen. www.fallschirmsportverband.de/ Deutscher Fallschirmsportverband, Überherrn. cited 2010 Apr 14.

32. El Kady D, Gilbert WM, Anderson J, Danielsen B, Towner D, Smith LH. Trauma druing Pregnancy: An analysis of maternal and fetal outcomes in a large population. AmJ Obstet gynecol. 2004;190:1661-8.

33. Engelhardt B. Reiten als Sport für Frauen und Mädchen in biologischer Sicht. Reiter-Revue 1965;9:592.

34. Erhard L. Sport und Schwangerschaft. Dissertation, LMU München: Medizinische Fakultät 2007. edoc.ub.uni-muenchen.de/8091/ cited 2010 Apr 14.

35. Friese K, Dudenhausen JW, Hoffmeister H, Kirschner W. BabyCare - die ersten Ergebnisse. Frauenarzt 2003;44:168-176.

36. Gesetz zum Schutz der erwerbstätigen Mutter (Mutterschutzgesetz - MuSchG).

Mutterschutzgesetz in der Fassung der Bekanntmachung vom 20. Juni 2002 (BGBI. I S. 2318), das zuletzt durch Artikel 14 des Gesetzes vom 17. März 2009 (BGBI. I S. 550) geändert worden ist.

37. Gesundheitsberichtserstattung des Bundes 2010. gbe-bund.de. cited 2010 Aug 1.

38. Goldenberg R, Culhane J, lams J, Romero R. Epidemiology and causes of preterm birth. The Lancet. 2008;371:75-84.

39. Grobecker C, Kreck-Roberg E, Sommer B. Bevölkerungsentwicklung 2005 in Wirtschaft und Statistik. Bundesamt für Statistik. 2007;1:45-47.

40. Haakstad LA, Voldner N, Henriksen T, Bø K. Why do pregnant women stop exercising in the third trimester? Acta obstetrica et gynaecologica Scandinavica 2009;88:1267-75.

41. Hammer R, Perkins J, Parr R. Exercise During the Childbearing Year. J Perinat Educ 2000 Winter;9:1-14.

42. Hatch M, Shu XO, McLean D, Levin B, Begg M, Reuss L, Susser M. Maternal Exercise during Pregnancy, Physical Fitness and Fetal Growth. A J Epidemiol. 1993;137:1105-1114.

43. Hegaard H, Hedegaard M, Damm P, Ottesen B, Petersson K, Henriksen TB. Leisure time physical activity is associated with a reduced risk of preterm delivery. Am J Obstet Gynecol. 2008;198:180.e1-180.e5.

44. Heipertz W., Der Sportarzt im Reitsport. Deutsche Zeitschrift für Sportmedizin. 2001;52:184-185.

45. Heipertz-Hengst C, Fischer S, Göres B, Sayn D, Homuth HP. Vibration Load of the Rider During Exercise on Horseback. $4^{\text {th }}$ Internat. Workshop on Animal Locomotion 2000, May 24-26, Vienna.

46. Heipertz-Hengst C. Fit fürs Pferd. Schwarzenbek 2002. 
47. Heleski CR, McGreevy PD, Kaiser LJ, Lavagnino M, Tans E, Bello N, Clayton HM. Effects on behaviour and rein tension on horses ridden with or without martingales and rein inserts. The Veterinary Journal. 209;181:56-62.

48. Henke T. Sportunfälle - Häufigkeit, Kosten, Prävention. Bochum 2002.

49. Hördegen KM. Wirbelsäule und Reiten. Schweiz, med. Wschr. 1975;105:668—675.

50. Hossain R, Harris T, Lohsoonthorn V, Williams M. Risk of preterm deliveri in relation to vaginal bleeding in early pregnancy. European Journal of Obstetrics \& Gynecology and Reproductive Biology. 2007;135:158-163.

51. Hübener E. Die Bewegungen von Pferderumpf und -rücken aus der Sicht des Reiters. Tierärztliche Umschau 2004;6:327-334.

52. Huch R, Erkkola R. Pregnancy and exercise - exercise and pregnancy. A short review. Br J Obstet Gynaecol 1990;97:208-214

53. Huch R. Glücklich schwanger von A-Z. Trias Stuttgart 2005.

54. Huch R. Physical activity at altitude in pregnancy. Semin Perinatol 1996;20:303-314

55. Huch R. Travel, sports and pregnancy. Geburtshilfe Frauenheilkd 1985;45:140-146

56. Jarrett J, Spellacy W. Jogging During Pregnancy: An Improved Outcome? Obstetrics and Gynecology. 1983;60:705.

57. Kardel KR. Effects of intense training during and after pregnancy in top-level athletes. Scand $\mathrm{J}$ Med Sci Sports 2005;15:79-86

58. Kim H, Lee SH, Kim SS, Yoo JH, Kim CH. The influence of maternal treadmill running durching pregnancy on short term memory and hippocampal cell survial in rat pulps. Int J Dev Neurosci. 2007;2:243-249.

59. Klein M, Hanssen P, MacWilliam L, Kaczorowski J, Johnson B. Determinants of vaginal-perineal integrity and pelvic floor functioning in childbirth. Am J Obst Gynecol 1997;176:403-410.

60. Kleinert J, Engelhard K, Sulprizio M. Sport in der Schwangerschaft: Epidemiologische Analysen und Zusammenhänge von körperlicher Aktivität mit psychosozialen und behavioralen Merkmalen vor und nach der Geburt. Abschlussbericht. Deutsche Sporthochschule Köln, Psychologisches Institut. Köln 2008.

61. Kleinert J, Sulprizio J. Wahrgenommener Informationsgrad als Bedingung von körperlicher Aktivität in der Schwangerschaft. In M. Knoll \& A. Woll (Hrsg.). Sport und Gesundheit in der Lebensspanne. Jahrestagung der dvs-Kommision Gesundheit 2008. Schriften der Deutschen Vereinigung für Sportwissenschaft 2008;174:293-397.

62. Kleinert J, Sulprizio M. Leistungssport und Schwangerschaft - epidemiologische und psychosoziale Betrachtungen. Leistungssport 2008;38:13-19.

63. Kleinert J, Sulprizio M. Leistungssport und Schwangerschaft - epidemiologische und psychosoziale Betrachtungen. Leistungssport 2008;38:13-19.

64. Kölbl $\mathrm{H}$. Make a cesarean sectio safer - have a vaginal delivery: Wie ist es jetzt wirklich mit dem Beckenboden und dem Geburtsmodus? J Urol Urogynäkol. 2003;10:17-21.

65. Kraft CN, Pennekamp PH, Becker U, Young M, Diedrich O, Lüring C, von Falkenhausen M. Magnetic resonance imaging findings of the lumbar spine in elite horseback risers: Correlations with back pain, body mass index, trunk/leg-length coefficient, an riding discipline. Am J Sports Med 2009;37:2205-2213.

66. Kraft CN, Urban N, Ilg A, Wallny T, Scharfstäadt A, Jäger M, Pennekamp PH. Einfluss der Reitdisziplin und -intensität auf die Inzidenz von Rückenschmerzen bei Reitsportlern. Sportverl Sportschad. 2007;21:1-5.

67. Kruger JA, Dieth HP, Murphy BA. Pelvic floor function in elite nulloparous athletes. Ultrasound Obstet Gynecol. 2007; Mai 14:1749-1753.

68. Kruger JA, Dietz P, Murphy BA. Pelvic floor function in elite nulliparous athletes. ultrasound Obstet Gynecol. 2007;May14:1749-1753. 
69. Lawson CC, Whelan EA, Hibert EN et al. Occupational factors and risk of preterm birth in nurses. Am J Obstet Gynecol 2009;200:51e1-51.e8.

70. Lehners B, Rath W. in Rath W, Friese K. Erkrankungen in der Schwangerschaft. Stuttgart 2005:263.

71. Luke B, Mamelle N, Keith L, et al. The association between occupational factors and preterm birth: a United States nurses' study. Am J Obstet Gynecol. 1995;173:849-862

72. Madsen M, Jørgensen T, Jensen ML, Juhl M, Olsen J, Andersen PK, Nybo Andersen A-M. Leisure time physical exercise during pregnancy and the risk of miscarriage: a study within the Danish National Birth Cohort. BJOG 2007;DOI:10-111/j14710428.2007.01496.x.

73. Mahoney B, Schwaitzberg S, Newton E. Trauma and Pregnancy. http://emedicine.medscape.com/article/435224-print.pdf. 2010 Feb (Update)

74. Melzer K, Schutz Y, Soehnchen M, Othenin-Girard V, Martinez de Tejada B, Irion O, Boulvain M, Kayser B. Effekcs of recommended levels of physical activity on pregnancy outcomes. Am J Obstet Gynecol. 2010;202:266.e1-266.e6.

75. Nawroth F, Dorn C, Ludwig M. „Richtiges“ Verhalten in der Frühschwangerschaft. Frauenarzt 2010;51:312-320.

76. Newton A, Nielsen AM. A Review of Horse-Related Injuries in a Rural Colorado Hospital: Implications for Outreach Education. Journal of Emergency Nursing. 2005;31:442-446.

77. Osthoff A. Vergleich der neuromuskulären Ansteuerungsfähigkeit von Reiterinnen und Nichtreiterinnen sowie Evaluation der Sportart Reiten zur Inkontinenzprävention in Bezug auf die physiotherapeutische Behandlung des Beckenbodens. Europa Fachschule Fresenius, Idstein 2008.

78. Oxynos K, Pildner von Steinburg S, Qattawi O, Ruckhäberle E, Rottenhuber J, Schneider KTM, Fischer T. Longitudinales, ambulantes Wehen-Langzeitmonitoring bei Schwangeren in der 26. bis 34. kpl. SSW unter körperlicher Belastung bzw. sexueller Aktivität, Z Geburtsh Neonatol 2003:207; Suppl 2: 589-5165.

79. Paringer C, Freerksen N, Sohn C, Maul H. Sport in der Schwangerschaft. gynäkol prax 2010;34:27-33.

80. Patteson SK, Snider CC, Meyer DS, Enderson BL, Armstrong JE, Whitaker GL, Carrol RC. The Consequences of High-risk Behaviors: Trauma During Pregnancy. J Trauma. 2007;62:1015-20.

81. Penttinen J, Erkkola R. Pregnancy in Endurance Athletes. Scand J Med Sci Sports. 2007;7:29227.

82. Pferdebetrieb 2000:5, Unfallzahlen landwirtschaftliche Berufsgenossenschaften Pferdewirte.

83. Pompeii L, Savitz D, Evenson K, Rogers B, McMahon M. Physical Exertion at Work and the Risk of Preterm Delivery and Small-for-Gestiational-Age Birth. Obstetrics \& Gynecology 2005;106:1279-1288.

84. Ramirez, Grimes R, Annegers J et al. Occupational Physical Activity and other Risk Factors for Preterm Birth eamong US Army Primigravidas. American Journal of Public Health 1990;80:728730.

85. Rathfelder FJ, Kleer P, Nachtkamp J, Paar O. Injuries in Horseback Riding - Incidence and Causes. Sportverletz Sportschaden. 1995;9:77-83.

86. Rayl J, Gibson J, Hickok D. A population-based case-control tudy of risk factors for breech presentation. Am J Obstet Gynecol. 1996:174:28-32.

87. Reimers A, Sulprizio M, Kleinert J. Schmerzerleben unter der Geburt in Abhängigkeit von Sportund Bewegungsaktivität. In M. Knoll \& A. Woll (Hrsg.). Sport und Gesundheit in der Lebensspanne. Jahrestagung der dvs-Kommision Gesundheit vom 10.-11.April 2008 in Bad Schönborn. (S. 298-302). Hamburg: Czwalina. 2008.

88. Reimers A, Sulprizio M, Kleinert J. Sport- und Bewegungsaktivitäten in der späten Schwangerschaft und Geburtsverlauf. In G. Sudeck, A. Conzelmann, K. Lehnert \& E.Gerlach (Hrsg.). Differentielle Sportpsychologie - Sportwissenschaftliche Persönlichkeitsforschung. Abstractband zur 40. Jahrestagung für Sportpsychologie vom 1.-3. Mai 2008 in Bern. Hamburg;2008:12. 
89. Rogers MS. Horse Riding During Pregnancy.

http://www.obgyn.net/displayarticle.asp?page=/pb/articles/horse_riding. Cited 2007, September 20.

90. Saling E, Al-Taie T, Schreiber M. Vermeidung sehr früher Frühgeburten - Aktueller Stand. Frauenarzt 2000;41:952-964.

91. Schäfer D, Pannek J. Measurement of pelvic floor function during physical activity: a feasibility study. Scandinavian journal of urology and nephrology. 2009;43:315-318.

92. Schlüssel MM, Souza EB, Reichenheim ME, Kac G. Physical activity during pregnancy and maternal-child health outcomes: a systematic literature review. Cadernos de Saúde Publica 2008;24:531-544.

93. Schroeder B. Blunt trauma in the pregnant women. Stanford Emergency Medicine 2007. http://scalpel.stanford.edu/ICU/OB\%20Trauma\%20powerpoint.ppt Cited 2010 May 25.

94. Seitz L. Schwangerschaft und Unfall. Archiv für orthopädische und Unfallchirurgie. 1933;34:177179.

95. Silver JR, Spinal injuries resulting from horse riding accidents. Spinal Cord 2002;40:264-271.

96. Strøm M, Mortensen EL, Halldorson TI, Osterdal ML, Olsen SF. Leisure-time physical activity in pragnancy and risk of postpartum depression: a prospective study in a large national birth cohort. Journal of Clinical Psychiatry 2009;70(:1707-14.

97. Sulprizio M, Kleinert J, Velde C Hartmann S. Sport und Schwangerschaft. Forschungsstand Folgen für die Praxis und Beratung. Sportverletzung - Sportschaden 2008;22:177-179.

98. Sulprizio M. Sport und Schwangerschaft - Mythen, Fakten, Empfehlungen. Vortrag FrauenSportWochen Essen 2009.

99. Sulprizio M., Kleinert J, Rojas S., Clemens C, Strüder H. Wohlbefinden und Leistungsfähigkeit von Hochschwangeren. In G. Sudeck, A. Conzelmann, K. Lehnert \& E. Gerlach (Hrsg.). Differentielle Sportpsychologie - Sportwissenschaftliche Persönlichkeitsforschung. Abstractband zur 40. Jahrestagung für Sportpsychologie vom 1.-3. Mai 2008 in Bern. Hamburg;2008:125.

100. Sulprizio, M, Kleinert J, Rojas S, Woschée U, Strüder H. Geburtsbegleitende Veränderungen von objektiver und wahrgenommener körperlicher Verfassung und ihr Einfluss auf postnatale Stimmung. Poster präsentiert auf der 41. Jahrestagung der Arbeitsgemeinschaft für Sportpsychologie in Leipzig. 2009.

101. Talas BB, Altinkaya SO, Talas H, Danisman N, Gungor T. Predictive Factors and Sort Term Fetal Outcomes of Breech Presentation: A Case Control Study. Taiwanese Journal of Obstetrics and Gynecology. 2008;47:402-407.

102. Wenzlaff, P., Zentrum für Qualität und Management im Gesundheitswesen, Einrichtung der Ärztekammer Niedersachsen. Persönliche Mitteilung. 5.11.2010.

103. Witte K, Schobesberger H, Peham C. Motion pattern analysis of gait in horseback riding by means of Principal Component Analysis. Hum Mov Sci 2009;28:394-405.

104. Zentrum für Qualität und Management im Gesundheitswesen, Ärztekammer Niedersachsen. Geburtshilfe Jahresauswertung 2004, Modul 16/1. Hannover 2005.

105. Ziegler M, Wollesen B, Mordziol C. Bewegungstherapie bei orthopädischen Problemen in der Schwangerschaft. Universität Hamburg, Fachbereich Sportwissenschaft 2002. www.rrz.unihamburg.de/.../Bewegungstherapie_Schwangerschaft_12-06-02.pdf . Cited 2010 April 19. 


\section{Danksagung}

\section{Die vorliegende Arbeit war nur möglich durch die Unterstützung zahlreicher Personen. Ihnen möchte ich meinen Dank aussprechen:}

Prof. Dr. med. habil. Klaus Friese möchte ich danken für die Überlassung des Themas und seine Ermutigung, das Projekt zu einem guten Ende zu bringen. Priv.-Doz. Dr. med. Mylonas danke ich für die kritische und geduldige Betreuung der Arbeit, Dres. Kirschner (FB+E) für die aufwändige Unterstützung und Beratung bei der Statistik, Dr. Christel Heipertz-Hengst vom Kuratorium Therapeutisches Reiten für ihre reiterliche und sportmedizinische Beratung und Ermutigung, Thieß Kaspareit von der Deutschen Akademie des Pferdes, Thomas Hartwig, im Jahr 2004/2005 Pressesprecher der Deutschen Reiterlichen Vereinigung, Hildegard Gehrmann von der Bundesvereinigung der Berufsreiter im DRFV e.V., One4Vision für die Programmierung der Online-Befragung, der Deutschen Gesellschaft für Gynäkologie und Geburtshilfe und dem Berufsverband der Frauenärzte für die Möglichkeit, auf die Untersuchung in der Zeitschrift „FRAUENARZT“ aufmerksam zu machen, Frau Prof. Debus und Frau Prof. Huch für ihre wichtigen Anregungen, Dr. A. Geisler vom STERN und Kerstin Niemann vom ST. GEORG für ihre wirkungsvolle Unterstützung bei der Öffentlichkeitsarbeit, Paul Wenzlaff vom Zentrum für Qualität und Management im Gesundheitswesen an der Ärztekammer Niedersachsen und Jürgen Strack von der Landwirtschaftlichen Berufsgenossenschaft Mittel- und Ostdeutschland für wichtige Hinweise. Dankbar bin ich auch der schwedischen Springreiterin Malin Baryard-Johnsson die im Jahr 2004, im 5. Monat schwanger, ein internationales Springen gewann und damit den Anstoß für diese Arbeit gegeben hat.

Mein Dank gilt auch den Frauen, die trotz ihrer vielfachen Belastungen an der aufwändigen Befragung teilgenommen haben und mir teilweise sehr emotionale Briefe geschrieben haben, und natürlich meinen eigenen Töchtern und meinem Lebenspartner, die mich nach allen Kräften unterstützt haben und denen ich viel Familienzeit vorenthalten habe, um diese Arbeit abzuschließen. 


\section{Curriculum vitae}

Susanna Kramarz

Oldenburgallee 60

14052 Berlin

1956

geboren in Leipzig

1981

Approbation als Ärztin, Freie Universität Berlin

1982

Wissenschaftliches Stipendium der Cusanus-Stifung

Medizinische Lehrkraft an Ausbildungsstätten für

Krankenschwestern und Musiktherapeuten

1984

Medizinpublizistin in Berlin, Schwerpunkt medizinische Fachpresse

(Deutsches Ärzteblatt, Ärzte Zeitung, Kassenarzt u.a.)

1985 - 1995 Redakteurin, später Chefredakteurin der ärztlichen Fachzeitschrift

THERAPIE DER GEGENWART im Verlag Urban \& Vogel, München

1993 Gründung und Chefredakteurin der Gesundheitszeitschrift

ELAN im Verlag Urban \& Vogel, München

1994 - $1995 \quad$ Lehrbeauftragte für den fachübergreifenden Kurs "Medizin in Zeitschriften“ am Institut für Publizistik, Freie Universität Berlin

1996 - 1998 Leitung medizinisch-wissenschaftliche Öffentlichkeitsarbeit der Gesundheits-Akademie Berlin

1998 - $1999 \quad$ Pressesprecherin der Zahnärztekammer Berlin

2001 - 2003 Stellvertretende Pressesprecherin und Online-Redakteurin im Bundesinstitut für Risikobewertung (BfR) 
seit 2003

Deutsche Gesellschaft für Gynäkologie und Geburtshilfe (DGGG)

Referentin der Schriftleitung (Zeitschrift FRAUENARZT)

Referentin des Leitlinienbeauftragten

2007

Gründung des Verlages S. Kramarz, Berlin

seit 2008

Herausgabe der Leitlinien der DGGG im Verlag S. Kramarz

seit Nov. 2010 Referentin für Presse und Öffentlichkeitsarbeit der DGGG

Persönliche Reiten

Interessen Musik

Alte Geschichte 


\section{Anhang}

\section{Vorabpublikationen}

Reiten in der Schwangerschaft - Frühgeburtlichkeit und Unfallrisiko

Vortrag anlässlich der Veranstaltung „Perinatologische Fortbildung im Vivantes-

Klinikum"

22. September 2010, Berlin

Horseback Riding During Pregnancy: No Significant Association With Preterm

Delivery

Susanna Kramarz, Renate Kirschner, Wolfgang Kirscher), Klaus Friese

Freier Vortrag anlässlich des 58. Kongresses der Deutschen Gesellschaft für

Gynäkologie und Geburtshilfe

Publiziert in Archives of Gynecology and Obstetrics. $58^{\text {th }}$ Congress of the German

Society of Gynecology and Obstetrics. Abstracts. FV-Geb 01.17, S8.

\section{Reiten in der Schwangerschaft - der Fragebogen}

\section{Tabellenband}

Tab. A1 Primiparae, ausführliche Dokumentation

Tab A2 Primiparae, verdichtet

Tab A3 Aborte, ausführliche Dokumentation

Tab A4 Primiparae, Teilnehmerinnen mit vorzeitigen Wehen, verdichtet

Tab A5 Primiparae, Entbindungen aus Beckenendlage, verdichtet

Tab A6 Geritten bis zur Entbindung in Terminnähe, BEL vs. SL, verdichtet

Tab A7A Primiparae - Spontanentbindungen vs. vaginal-operative Entbindungen

Tab A7B Primiparae - Spontanentbindungen vs. sekundäre Sectiones

Tab. A8 Unfälle beim Reiten und beim Umgang mit dem Pferd, verdichtet 
Reiten in der Schwangerschaft - Primiparae

Anhang Tab. A1

Entbindungen ab $37+0$ vs. Entbindungen bis $36+6$

\begin{tabular}{|c|c|c|c|c|c|c|c|c|}
\hline & $\begin{array}{l}\text { Primi- } \\
\text { parae }\end{array}$ & $\begin{array}{l}\text { Pro- } \\
\text { zent }\end{array}$ & $\begin{array}{c}\mathrm{PP} a b \\
37+0\end{array}$ & $\begin{array}{l}\text { Pro- } \\
\text { zent }\end{array}$ & $\begin{array}{l}\text { PP bis } \\
36+6\end{array}$ & Pro-zent & & \\
\hline$n=$ & 1122 & & 1052 & & 70 & & & \\
\hline Definitionen & & & & & & & Wurzel S & Signifikanz \\
\hline \multicolumn{9}{|l|}{ Alter zu Beginn des SS } \\
\hline$<18$ Jahre & 8 & 0,7 & 8 & $0,8 \%$ & 0 & $0,0 \%$ & 2,84 & ns \\
\hline 18 bis 24 Jahre & 162 & 14,4 & 151 & $14,4 \%$ & 11 & $15,7 \%$ & 0,30 & ns \\
\hline 25-29 Jahre & 433 & 38,6 & 405 & $38,5 \%$ & 28 & $40,0 \%$ & 0,25 & ns \\
\hline 30-34 Jahre & 407 & 36,3 & 384 & $36,5 \%$ & 23 & $32,9 \%$ & 0,63 & ns \\
\hline 35-39 Jahre & 103 & 9,2 & 95 & $9,0 \%$ & 8 & $11,4 \%$ & 0,61 & ns \\
\hline 40 Jahre und mehr & 9 & 0,8 & 9 & $0,9 \%$ & 0 & $0,0 \%$ & 3,01 & ns \\
\hline Mittelwert & 29,1 & & 29,1 & & 29,3 & & 0,35 & ns \\
\hline \multicolumn{9}{|l|}{ BMI } \\
\hline Untergewicht $<20$ & 219 & 19,5 & 204 & $19,4 \%$ & 14 & $20,0 \%$ & 0,12 & ns \\
\hline Normalgewicht 20-24,9 & 730 & 65,1 & 683 & $64,9 \%$ & 48 & $68,6 \%$ & 0,64 & ns \\
\hline Übergewicht > 25 & 172 & 15,3 & 164 & $15,6 \%$ & 8 & $11,4 \%$ & 1,05 & ns \\
\hline keine Angabe & 0 & 0,0 & 1 & $0,0 \%$ & 0 & $0,0 \%$ & & \\
\hline Summe & 1121 & 99,9 & 1052 & $99,9 \%$ & 70 & $100,0 \%$ & & \\
\hline \multicolumn{9}{|l|}{ frühere Gravidität } \\
\hline keine & 1009 & 89,9 & 950 & $90,3 \%$ & 59 & $84,3 \%$ & 1,35 & ns \\
\hline 1 & 95 & 8,5 & 86 & $8,2 \%$ & 9 & $12,9 \%$ & 1,15 & ns \\
\hline 2 & 14 & 1,2 & 12 & $1,1 \%$ & 2 & $2,9 \%$ & 0,85 & ns \\
\hline 3 & 3 & 0,3 & 3 & $0,3 \%$ & 0 & $0,0 \%$ & 1,73 & ns \\
\hline 4 & 1 & 0,1 & 1 & $0,1 \%$ & 0 & $0,0 \%$ & 1,00 & ns \\
\hline 5 & 0 & 0,0 & 0 & $0,0 \%$ & 0 & $0,0 \%$ & & \\
\hline mehr als 5 & 0 & 0,0 & 0 & $0,0 \%$ & 0 & $0,0 \%$ & & \\
\hline mindestens 1 & 113 & 10,1 & 102 & $9,7 \%$ & 11 & $15,7 \%$ & 1,35 & ns \\
\hline \multicolumn{9}{|l|}{ frühere Geburten } \\
\hline keine & 1122 & 100,0 & 1052 & $100,0 \%$ & 70 & $100,0 \%$ & & \\
\hline 1 & 0 & 0,0 & 0 & $0,0 \%$ & 0 & $0,0 \%$ & & \\
\hline 2 & 0 & 0,0 & 0 & $0,0 \%$ & 0 & $0,0 \%$ & & \\
\hline 3 & 0 & 0,0 & 0 & $0,0 \%$ & 0 & $0,0 \%$ & & \\
\hline 4 & 0 & 0,0 & 0 & $0,0 \%$ & 0 & $0,0 \%$ & & \\
\hline 5 & 0 & 0,0 & 0 & $0,0 \%$ & 0 & $0,0 \%$ & & \\
\hline mehr als 5 & 0 & 0,0 & 0 & $0,0 \%$ & 0 & $0,0 \%$ & & \\
\hline mindestens 1 & 0 & 0,0 & 0 & $0,0 \%$ & 0 & $0,0 \%$ & & \\
\hline \multicolumn{9}{|l|}{ Frühere Kompl.: } \\
\hline gyn. Operation & 69 & 6,1 & 64 & $6,1 \%$ & 5 & $7,1 \%$ & 0,33 & ns \\
\hline $\begin{array}{l}\text { Krankenhausaufenthalt } \\
\text { mind. } 2 \text { Wochen vor Sws }\end{array}$ & 9 & 0,8 & 8 & $0,8 \%$ & 1 & $1,4 \%$ & 0,46 & ns \\
\hline \multicolumn{9}{|l|}{ Schulabschluss } \\
\hline $\begin{array}{l}\text { Schule noch nicht } \\
\text { beendet }\end{array}$ & 11 & 1,0 & 10 & $1,0 \%$ & 1 & $1,4 \%$ & 0,33 & ns \\
\hline $\begin{array}{l}\text { Schule beendet ohne } \\
\text { Abschluss }\end{array}$ & 1 & 0,1 & 1 & $0,1 \%$ & 0 & $0,0 \%$ & 1,00 & ns \\
\hline $\begin{array}{l}\text { Haupt/ } \\
\text { Volksschulabschluss }\end{array}$ & 55 & 4,9 & 51 & $4,8 \%$ & 4 & $5,7 \%$ & 0,30 & ns \\
\hline Realschulabschluss & 318 & 28,3 & 295 & $28,0 \%$ & 23 & $32,9 \%$ & 0,83 & ns \\
\hline $\begin{array}{l}\text { Abschluss } \\
\text { Polytechnische } \\
\text { Oberschule }\end{array}$ & 23 & 2,0 & 19 & $1,8 \%$ & 4 & $5,7 \%$ & 1,39 & ns \\
\hline Fachhochschulreife & 141 & 12,6 & 133 & $12,6 \%$ & 8 & $11,4 \%$ & 0,31 & ns \\
\hline
\end{tabular}


Reiten in der Schwangerschaft - Primiparae

Anhang Tab. A1

Entbindungen ab $37+0$ vs. Entbindungen bis $36+6$

\begin{tabular}{|c|c|c|c|c|c|c|c|c|}
\hline & $\begin{array}{l}\text { Primi- } \\
\text { parae }\end{array}$ & $\begin{array}{l}\text { Pro- } \\
\text { zent }\end{array}$ & $\begin{array}{c}\text { PP ab } \\
37+0\end{array}$ & $\begin{array}{l}\text { Pro- } \\
\text { zent }\end{array}$ & $\begin{array}{l}\text { PP bis } \\
36+6\end{array}$ & Pro-zent & & \\
\hline$n=$ & 1122 & & 1052 & & 70 & & & \\
\hline Definitionen & & & & & & & Wurzel S & Signifikanz \\
\hline \multicolumn{9}{|l|}{$\begin{array}{l}\text { Sportlicher } \\
\text { Trainingszustand vor } \\
\text { der Schwangerschaft }\end{array}$} \\
\hline $\begin{array}{l}\text { ausgezeichnet (täglich } \\
\text { mind. } 1 \mathrm{~h} \text { Sport) }\end{array}$ & 281 & 25,0 & 265 & $25,2 \%$ & 16 & $22,9 \%$ & 0,45 & ns \\
\hline $\begin{array}{l}\text { sehr gut (4h und mehr } \\
\text { Sport pro Woche) }\end{array}$ & 352 & 31,4 & 329 & $31,3 \%$ & 23 & $32,9 \%$ & 0,27 & ns \\
\hline $\begin{array}{l}\text { gut (2-4h Sport pro } \\
\text { Woche) }\end{array}$ & 348 & 31,0 & 331 & $31,5 \%$ & 17 & $24,3 \%$ & 1,35 & ns \\
\hline $\begin{array}{l}\text { mäßig (1-2 h Sport pro } \\
\text { Woche) }\end{array}$ & 107 & 9,5 & 97 & $9,2 \%$ & 10 & $14,3 \%$ & 1,18 & ns \\
\hline $\begin{array}{l}\text { nicht besonders (weniger } \\
\text { als } 1 \mathrm{~h} \text { Sport pro Wo.) }\end{array}$ & 34 & 3,0 & 30 & $2,9 \%$ & 4 & $5,7 \%$ & 1,01 & ns \\
\hline \multicolumn{9}{|l|}{$\begin{array}{l}\text { Sportarten vor der } \\
\text { Schwangerschaft }\end{array}$} \\
\hline $\begin{array}{l}\text { keine Sportarten } \\
\text { zusätzlich zum Reiten }\end{array}$ & 418 & 37,3 & 388 & $36,9 \%$ & 30 & $42,9 \%$ & 0,98 & ns \\
\hline $\begin{array}{l}\text { Insgesamt sportl. aktiv } \\
\text { zusätzlich zum Reiten }\end{array}$ & 704 & 62,7 & 664 & $63,1 \%$ & 40 & $57,1 \%$ & 0,98 & ns \\
\hline $\begin{array}{l}\text { Ausdauerbetonte } \\
\text { Sportarten }\end{array}$ & 498 & 44,4 & 473 & $45,0 \%$ & 25 & $35,7 \%$ & 1,56 & ns \\
\hline Kraftbetonte Sportarten & 84 & 7,5 & 79 & $7,5 \%$ & 5 & $7,1 \%$ & 0,12 & ns \\
\hline Gymnastische Sportarten & 130 & 11,6 & 120 & $11,4 \%$ & 10 & $14,3 \%$ & 0,67 & ns \\
\hline $\begin{array}{l}\text { Fun- und } \\
\text { Risikosportarten }\end{array}$ & 175 & 15,6 & 168 & $16,0 \%$ & 7 & $10,0 \%$ & 1,59 & ns \\
\hline Spielsportarten & 76 & 6,8 & 74 & $7,0 \%$ & 2 & $2,9 \%$ & 1,95 & ns \\
\hline nur 1 Sportart & 485 & 43,2 & 453 & $43,1 \%$ & 32 & $45,7 \%$ & 0,43 & ns \\
\hline 2 Sportarten & 181 & 16,1 & 174 & $16,5 \%$ & 7 & $10,0 \%$ & 1,74 & ns \\
\hline 3 oder 4 Sportarten & 38 & 3,4 & 37 & $3,5 \%$ & 1 & $1,4 \%$ & 1,37 & ns \\
\hline \multicolumn{9}{|l|}{ Leistungsstand Reiten } \\
\hline Ungeübte Reiterin & 24 & 2,1 & 21 & $2,0 \%$ & 3 & $4,3 \%$ & 0,93 & ns \\
\hline Geübte Reiterin & 737 & 65,7 & 693 & $65,9 \%$ & 44 & $62,9 \%$ & 0,51 & ns \\
\hline $\begin{array}{l}\text { Leistungssportlerin im } \\
\text { gehobenen Bereich }\end{array}$ & 315 & 28,1 & 294 & $27,9 \%$ & 21 & $30,0 \%$ & 0,36 & ns \\
\hline Berufsreiterin & 46 & 4,1 & 44 & $4,2 \%$ & 2 & $2,9 \%$ & 0,64 & ns \\
\hline \multicolumn{9}{|l|}{ Leistungsklasse } \\
\hline ohne & 801 & 71,4 & 753 & $71,6 \%$ & 48 & $68,6 \%$ & 0,53 & ns \\
\hline LK 1 & 2 & 0,2 & 1 & $0,1 \%$ & 1 & $1,4 \%$ & 0,94 & ns \\
\hline LK 2 & 8 & 0,7 & 8 & $0,8 \%$ & 0 & $0,0 \%$ & 2,84 & ns \\
\hline LK 3 & 30 & 2,7 & 27 & $2,6 \%$ & 3 & $4,3 \%$ & 0,70 & ns \\
\hline LK 4 & 89 & 7,9 & 76 & $7,2 \%$ & 13 & $18,6 \%$ & 2,41 & $\mathrm{~s}$ \\
\hline LK 5 & 105 & 9,4 & 102 & $9,7 \%$ & 3 & $4,3 \%$ & 2,09 & ns \\
\hline LK 6 & 16 & 1,4 & 16 & $1,5 \%$ & 0 & $0,0 \%$ & 4,03 & ns \\
\hline & & & & & & & & \\
\hline & & & & & & & & \\
\hline & & & & & & & & \\
\hline & & & & & & & & \\
\hline
\end{tabular}


Reiten in der Schwangerschaft - Primiparae

Anhang Tab. A1

Entbindungen ab $37+0$ vs. Entbindungen bis $36+6$

\begin{tabular}{|c|c|c|c|c|c|c|c|c|}
\hline & $\begin{array}{l}\text { Primi- } \\
\text { parae }\end{array}$ & $\begin{array}{l}\text { Pro- } \\
\text { zent }\end{array}$ & $\begin{array}{c}\text { PP ab } \\
37+0\end{array}$ & $\begin{array}{l}\text { Pro- } \\
\text { zent }\end{array}$ & $\begin{array}{l}\text { PP bis } \\
36+6\end{array}$ & Pro-zent & & \\
\hline$n=$ & 1122 & & 1052 & & 70 & & & \\
\hline Definitionen & & & & & & & Wurzel S & Signifikanz \\
\hline $\begin{array}{l}\text { nur gelegentlich oder im } \\
\text { Urlaub }\end{array}$ & 13 & 1,2 & 10 & $1,0 \%$ & 3 & $4,3 \%$ & 1,37 & ns \\
\hline 1x pro Woche & 22 & 2,0 & 20 & $1,9 \%$ & 2 & $2,9 \%$ & 0,47 & ns \\
\hline 2x pro Woche & 103 & 9,2 & 100 & $9,5 \%$ & 3 & $4,3 \%$ & 2,02 & ns \\
\hline $3-5 x$ pro Woche & 556 & 49,6 & 517 & $49,1 \%$ & 39 & $55,7 \%$ & 1,07 & ns \\
\hline täglich & 428 & 38,1 & 405 & $38,5 \%$ & 23 & $32,9 \%$ & 0,97 & ns \\
\hline \multicolumn{9}{|l|}{$\begin{array}{l}\text { Tägliche Reitdauer vor } \\
\text { der SWS }\end{array}$} \\
\hline $1 \mathrm{~h}$ & 720 & 64,2 & 679 & $64,5 \%$ & 41 & $58,6 \%$ & 0,98 & ns \\
\hline $2-3 \mathrm{~h}$ & 361 & 32,2 & 333 & $31,7 \%$ & 28 & $40,0 \%$ & 1,38 & ns \\
\hline $4 \mathrm{~h}$ und mehr & 41 & 3,7 & 40 & $3,8 \%$ & 1 & $1,4 \%$ & 1,55 & ns \\
\hline \multicolumn{9}{|l|}{ Dressur } \\
\hline nicht geritten & 162 & 14,4 & 154 & $14,6 \%$ & 8 & $11,4 \%$ & 0,81 & ns \\
\hline leichte Belastung & 125 & 11,1 & 117 & $11,1 \%$ & 8 & $11,4 \%$ & 0,08 & ns \\
\hline mittlere Belastung & 309 & 27,5 & 286 & $27,2 \%$ & 23 & $32,9 \%$ & 0,98 & ns \\
\hline $\begin{array}{l}\text { leistungsorientiertes } \\
\text { Reiten }\end{array}$ & 448 & 39,9 & 425 & $40,4 \%$ & 23 & $32,9 \%$ & 1,30 & ns \\
\hline Spitzensport & 77 & 6,9 & 70 & $6,7 \%$ & 7 & $10,0 \%$ & 0,91 & ns \\
\hline \multicolumn{9}{|l|}{ Springen } \\
\hline nicht geritten & 512 & 45,6 & 482 & $45,8 \%$ & 30 & $42,9 \%$ & 0,48 & ns \\
\hline leichte Belastung & 228 & 20,3 & 210 & $20,0 \%$ & 18 & $25,7 \%$ & 1,07 & ns \\
\hline mittlere Belastung & 186 & 16,6 & 178 & $16,9 \%$ & 8 & $11,4 \%$ & 1,38 & ns \\
\hline $\begin{array}{l}\text { leistungsorientiertes } \\
\text { Reiten }\end{array}$ & 171 & 15,2 & 158 & $15,0 \%$ & 13 & $18,6 \%$ & 0,74 & ns \\
\hline Spitzensport & 25 & 2,2 & 24 & $2,3 \%$ & 1 & $1,4 \%$ & 0,57 & ns \\
\hline \multicolumn{9}{|l|}{ Ausreiten } \\
\hline nicht geritten & 44 & 3,9 & 42 & $4,0 \%$ & 2 & $2,9 \%$ & 0,55 & ns \\
\hline leichte Belastung & 240 & 21,4 & 221 & $21,0 \%$ & 19 & $27,1 \%$ & 1,12 & ns \\
\hline mittlere Belastung & 563 & 50,2 & 528 & $50,2 \%$ & 35 & $50,0 \%$ & 0,03 & ns \\
\hline $\begin{array}{l}\text { leistungsorientiertes } \\
\text { Reiten }\end{array}$ & 244 & 21,7 & 232 & $22,1 \%$ & 12 & $17,1 \%$ & 1,05 & $\mathrm{~ns}$ \\
\hline Spitzensport & 31 & 2,8 & 29 & $2,8 \%$ & 2 & $2,9 \%$ & 0,05 & ns \\
\hline \multicolumn{9}{|l|}{ Gelände / Vielseitigkeit } \\
\hline nicht geritten & 712 & 63,5 & 664 & $63,1 \%$ & 48 & $68,6 \%$ & 0,95 & ns \\
\hline leichte Belastung & 133 & 11,9 & 123 & $11,7 \%$ & 10 & $14,3 \%$ & 0,60 & ns \\
\hline mittlere Belastung & 180 & 16,0 & 174 & $16,5 \%$ & 6 & $8,6 \%$ & 2,25 & $\mathrm{~s}$ \\
\hline $\begin{array}{l}\text { leistungsorientiertes } \\
\text { Reiten }\end{array}$ & 89 & 7,9 & 83 & $7,9 \%$ & 6 & $8,6 \%$ & 0,20 & ns \\
\hline Spitzensport & 8 & 0,7 & 8 & $0,8 \%$ & 0 & $0,0 \%$ & 2,84 & ns \\
\hline \multicolumn{9}{|l|}{ Gangpferde } \\
\hline nicht geritten & 1026 & 91,4 & 964 & $91,6 \%$ & 62 & $88,6 \%$ & 0,79 & ns \\
\hline leichte Belastung & 23 & 2,0 & 19 & $1,8 \%$ & 4 & $5,7 \%$ & 1,39 & ns \\
\hline mittlere Belastung & 36 & 3,2 & 34 & $3,2 \%$ & 2 & $2,9 \%$ & 0,18 & $\mathrm{~ns}$ \\
\hline $\begin{array}{l}\text { leistungsorientiertes } \\
\text { Reiten }\end{array}$ & 27 & 2,4 & 25 & $2,4 \%$ & 2 & $2,9 \%$ & 0,23 & ns \\
\hline Spitzensport & 10 & 0,9 & 10 & $1,0 \%$ & 0 & $0,0 \%$ & 3,18 & ns \\
\hline \multicolumn{9}{|l|}{ Western } \\
\hline nicht geritten & 960 & 85,6 & 901 & $85,6 \%$ & 59 & $84,3 \%$ & 0,30 & ns \\
\hline
\end{tabular}


Reiten in der Schwangerschaft - Primiparae

Anhang Tab. A1

Entbindungen ab $37+0$ vs. Entbindungen bis $36+6$

\begin{tabular}{|c|c|c|c|c|c|c|c|c|}
\hline & $\begin{array}{l}\text { Primi- } \\
\text { parae }\end{array}$ & $\begin{array}{l}\text { Pro- } \\
\text { zent }\end{array}$ & $\begin{array}{c}\text { PP ab } \\
37+0\end{array}$ & $\begin{array}{l}\text { Pro- } \\
\text { zent }\end{array}$ & $\begin{array}{c}\text { PP bis } \\
36+6\end{array}$ & Pro-zent & & \\
\hline$n=$ & 1122 & & 1052 & & 70 & & & \\
\hline Definitionen & & & & & & & Wurzel S & Signifikanz \\
\hline leichte Belastung & 44 & 3,9 & 43 & $4,1 \%$ & 1 & $1,4 \%$ & 1,72 & ns \\
\hline mittlere Belastung & 61 & 5,4 & 56 & $5,3 \%$ & 5 & $7,1 \%$ & 0,58 & ns \\
\hline $\begin{array}{l}\text { leistungsorientiertes } \\
\text { Reiten }\end{array}$ & 54 & 4,8 & 49 & $4,7 \%$ & 5 & $7,1 \%$ & 0,79 & ns \\
\hline Spitzensport & 3 & 0,3 & 3 & $0,3 \%$ & 0 & $0,0 \%$ & 1,73 & ns \\
\hline \multicolumn{9}{|l|}{ Distanzreiten } \\
\hline nicht geritten & 1024 & 91,3 & 961 & $91,3 \%$ & 63 & $90,0 \%$ & 0,37 & ns \\
\hline leichte Belastung & 33 & 2,9 & 32 & $3,0 \%$ & 1 & $1,4 \%$ & 1,07 & ns \\
\hline mittlere Belastung & 36 & 3,2 & 33 & $3,1 \%$ & 3 & $4,3 \%$ & 0,46 & ns \\
\hline $\begin{array}{l}\text { leistungsorientiertes } \\
\text { Reiten }\end{array}$ & 23 & 2,0 & 21 & $2,0 \%$ & 2 & $2,9 \%$ & 0,42 & ns \\
\hline Spitzensport & 6 & 0,5 & 5 & $0,5 \%$ & 1 & $1,4 \%$ & 0,66 & ns \\
\hline \multicolumn{9}{|l|}{ Rennsport } \\
\hline nicht geritten & 1105 & 98,5 & 1036 & $98,5 \%$ & 69 & $98,6 \%$ & 0,06 & ns \\
\hline leichte Belastung & 11 & 1,0 & 11 & $1,0 \%$ & 0 & $0,0 \%$ & 3,33 & ns \\
\hline mittlere Belastung & 2 & 0,2 & 2 & $0,2 \%$ & 0 & $0,0 \%$ & 1,42 & ns \\
\hline $\begin{array}{l}\text { leistungsorientiertes } \\
\text { Reiten }\end{array}$ & 3 & 0,3 & 2 & $0,2 \%$ & 1 & $1,4 \%$ & 0,87 & ns \\
\hline Spitzensport & 1 & 0,1 & 1 & $0,1 \%$ & 0 & $0,0 \%$ & 1,00 & ns \\
\hline \multicolumn{9}{|l|}{$\begin{array}{l}\text { Information des } \\
\text { Frauenarztes }\end{array}$} \\
\hline $\begin{array}{l}\text { ja, bereits vor der } \\
\text { Schwangerschaft }\end{array}$ & 377 & 33,6 & 346 & $32,9 \%$ & 31 & $44,3 \%$ & 1,86 & ns \\
\hline $\begin{array}{l}\text { ja, zu Beginn der } \\
\text { Schwangerschaft }\end{array}$ & 595 & 53,0 & 564 & $53,6 \%$ & 31 & $44,3 \%$ & 1,52 & ns \\
\hline $\begin{array}{l}\text { ja, im späteren Verlauf } \\
\text { der Schwangerschaft }\end{array}$ & 64 & 5,7 & 60 & $5,7 \%$ & 4 & $5,7 \%$ & 0,00 & ns \\
\hline nein & 86 & 7,7 & 82 & $7,8 \%$ & 4 & $5,7 \%$ & 0,72 & ns \\
\hline \multicolumn{9}{|l|}{$\begin{array}{l}\text { Empfehlung des } \\
\text { Frauenarztes }\end{array}$} \\
\hline hat abgeraten & 152 & 13,5 & 143 & $13,6 \%$ & 9 & $12,9 \%$ & 0,18 & ns \\
\hline $\begin{array}{l}\text { Entscheidung der } \\
\text { Schwangeren überlassen }\end{array}$ & 683 & 60,9 & 640 & $60,8 \%$ & 43 & $61,4 \%$ & 0,10 & ns \\
\hline Weiterreiten empfohlen & 201 & 17,9 & 187 & $17,8 \%$ & 14 & $20,0 \%$ & 0,45 & ns \\
\hline $\begin{array}{l}\text { Frauenarzt war nicht } \\
\text { informiert }\end{array}$ & 86 & 7,7 & 82 & $7,8 \%$ & 4 & $5,7 \%$ & 0,72 & ns \\
\hline \multicolumn{9}{|l|}{$\begin{array}{l}\text { Nach Feststellung der } \\
\text { Sws weitergeritten }\end{array}$} \\
\hline $\mathrm{Ja}$ & 1039 & 92,6 & 973 & $92,5 \%$ & 66 & $94,3 \%$ & 0,62 & ns \\
\hline $\begin{array}{l}\text { und zwar bis zur } \\
\text { Schwangerschaftswoche }\end{array}$ & 28,92 & & 29,20 & & 24,73 & & 19,3 (T-Wert) & $\mathrm{s}$ \\
\hline St-Abweichung & & & 1,77 & & 1,88 & & & \\
\hline Nein & 81 & 7,2 & 77 & $7,3 \%$ & 4 & $5,7 \%$ & 0,56 & ns \\
\hline $\begin{array}{l}\text { aufgehört in } \\
\text { Schwangerschaftswoche }\end{array}$ & 8 & & 8 & & 0 & & & o.A. \\
\hline
\end{tabular}


Reiten in der Schwangerschaft - Primiparae

Anhang Tab. A1

Entbindungen ab $37+0$ vs. Entbindungen bis $36+6$

\begin{tabular}{|c|c|c|c|c|c|c|c|c|}
\hline & $\begin{array}{l}\text { Primi- } \\
\text { parae }\end{array}$ & $\begin{array}{l}\text { Pro- } \\
\text { zent }\end{array}$ & $\begin{array}{c}\mathrm{PP} a b \\
37+0\end{array}$ & $\begin{array}{l}\text { Pro- } \\
\text { zent }\end{array}$ & $\begin{array}{l}\text { PP bis } \\
36+6\end{array}$ & Pro-zent & & \\
\hline$n=$ & 1122 & & 1052 & & 70 & & & \\
\hline Definitionen & & & & & & & Wurzel S & Signifikanz \\
\hline \multicolumn{9}{|l|}{$\begin{array}{l}\text { Beschwerden im } \\
\text { Zusammenhang mit } \\
\text { dem Reiten }\end{array}$} \\
\hline $\begin{array}{l}\text { 1. Trimenon harter Bauch } \\
\text { od. Ziehen im Unterleib }\end{array}$ & 43 & 3,8 & 39 & $3,7 \%$ & 4 & $5,7 \%$ & 0,71 & ns \\
\hline $\begin{array}{l}\text { 2. Trimenon } \\
\text { Beschwerden }\end{array}$ & 81 & 7,2 & 76 & $7,2 \%$ & 5 & $7,1 \%$ & 0,03 & ns \\
\hline $\begin{array}{l}\text { 1.und 2. Trimenon } \\
\text { Beschwerden }\end{array}$ & 19 & 1,7 & 15 & $1,4 \%$ & 4 & $5,7 \%$ & 1,53 & ns \\
\hline $\begin{array}{l}\text { 1. und 3. Trimenon } \\
\text { Beschwerden }\end{array}$ & 1 & 0,1 & 1 & $0,1 \%$ & 0 & $0,0 \%$ & 1,00 & ns \\
\hline $\begin{array}{l}\text { 2. und 3. Trimenon } \\
\text { Beschwerden }\end{array}$ & 32 & 2,9 & 32 & $3,0 \%$ & 0 & $0,0 \%$ & 5,74 & ns \\
\hline $\begin{array}{l}\text { 1.,2.,3. Trimenon } \\
\text { Beschwerden }\end{array}$ & 9 & 0,8 & 8 & $0,8 \%$ & 1 & $1,4 \%$ & 0,46 & ns \\
\hline $\begin{array}{l}\text { Gesamt keine } \\
\text { Beschwerden }\end{array}$ & 813 & 72,5 & 763 & $72,5 \%$ & 50 & $71,4 \%$ & 0,20 & ns \\
\hline Gesamt Beschwerden & 309 & 27,5 & 289 & $27,5 \%$ & 20 & $28,6 \%$ & 0,20 & ns \\
\hline \multicolumn{9}{|l|}{$\begin{array}{l}\text { Ich habe mit dem } \\
\text { Reiten aufgehört, }\end{array}$} \\
\hline $\begin{array}{l}\text { erst als das Reiten } \\
\text { unbequem wurde }\end{array}$ & 629 & 56,1 & 591 & $56,2 \%$ & 38 & $54,3 \%$ & 0,31 & ns \\
\hline $\begin{array}{l}\text { weil ich Angst hatte, mir } \\
\text { oder dem Kind zu } \\
\text { schaden }\end{array}$ & 261 & 23,3 & 244 & $23,2 \%$ & 17 & $24,3 \%$ & 0,21 & ns \\
\hline $\begin{array}{l}\text { weil ich vorzeitige } \\
\text { Wehen, Blutungen oder } \\
\text { andere Probleme mit der } \\
\text { Schwangerschaft } \\
\text { bekommen habe }\end{array}$ & 88 & 7,8 & 83 & $7,9 \%$ & 5 & $7,1 \%$ & 0,23 & ns \\
\hline $\begin{array}{l}\text { weil der Frauenarzt oder } \\
\text { eine andere Person mir } \\
\text { das Aufhören dringend } \\
\text { nahe gelegt hat }\end{array}$ & 81 & 7,2 & 71 & $6,7 \%$ & 10 & $14,3 \%$ & 1,77 & ns \\
\hline $\begin{array}{l}\text { weil ich krank geworden } \\
\text { bin }\end{array}$ & 29 & 2,6 & 25 & $2,4 \%$ & 4 & $5,7 \%$ & 1,19 & ns \\
\hline andere Gründe & 207 & 18,4 & 193 & $18,3 \%$ & 14 & $20,0 \%$ & 0,34 & ns \\
\hline $\begin{array}{l}\text { bis zur Geburt } \\
\text { weitergeritten }\end{array}$ & 132 & 11,8 & 127 & $12,1 \%$ & 5 & $7,1 \%$ & 1,52 & ns \\
\hline $\begin{array}{l}\text { erst als das Reiten } \\
\text { unbequem wurde - allein }\end{array}$ & 442 & 39,4 & 416 & $39,5 \%$ & 26 & $37,1 \%$ & 0,40 & ns \\
\hline $\begin{array}{l}\text { weil ich vorzeitige } \\
\text { Wehen, etc. bekommen } \\
\text { habe - allein }\end{array}$ & 43 & 3,8 & 41 & $3,9 \%$ & 2 & $2,9 \%$ & 0,50 & ns \\
\hline $\begin{array}{l}\text { weil ich Angst hatte, mir } \\
\text { oder dem Kind zu } \\
\text { schaden - allein }\end{array}$ & 100 & 8,9 & 94 & $8,9 \%$ & 6 & $8,6 \%$ & 0,11 & ns \\
\hline
\end{tabular}


Reiten in der Schwangerschaft - Primiparae

Anhang Tab. A1

Entbindungen ab $37+0$ vs. Entbindungen bis $36+6$

\begin{tabular}{|c|c|c|c|c|c|c|c|c|}
\hline & $\begin{array}{l}\text { Primi- } \\
\text { parae }\end{array}$ & $\begin{array}{l}\text { Pro- } \\
\text { zent }\end{array}$ & $\begin{array}{c}\mathrm{PP} a b \\
37+0\end{array}$ & $\begin{array}{l}\text { Pro- } \\
\text { zent }\end{array}$ & $\begin{array}{l}\text { PP bis } \\
36+6\end{array}$ & Pro-zent & & \\
\hline$n=$ & 1122 & & 1052 & & 70 & & & \\
\hline Definitionen & & & & & & & Wurzel S & Signifikanz \\
\hline $\begin{array}{l}\text { weil jemand mir das } \\
\text { Aufhören dringend nahe } \\
\text { gelegt hat - allein }\end{array}$ & 20 & 1,8 & 18 & $1,7 \%$ & 2 & $2,9 \%$ & 0,56 & ns \\
\hline $\begin{array}{l}\text { Angst hatte UND } \\
\text { Aufhören nahe gelegt }\end{array}$ & 21 & 1,9 & 19 & $1,8 \%$ & 2 & $2,9 \%$ & 0,52 & ns \\
\hline $\begin{array}{l}\text { weil ich krank geworden } \\
\text { bin - allein }\end{array}$ & 11 & 1,0 & 9 & $0,9 \%$ & 2 & $2,9 \%$ & 1,00 & ns \\
\hline zwei Gründe angegeben & 276 & 24,6 & 256 & $24,3 \%$ & 20 & $28,6 \%$ & 0,76 & ns \\
\hline \multicolumn{9}{|l|}{$\begin{array}{l}\text { Trotz Warnzeichen } \\
\text { weitergeritten ... }\end{array}$} \\
\hline ja & 71 & 6,3 & 68 & $6,5 \%$ & 3 & $4,3 \%$ & 0,86 & ns \\
\hline nein & 909 & 81,0 & 848 & $80,6 \%$ & 61 & $87,1 \%$ & 1,56 & ns \\
\hline keine Angabe & 142 & 12,7 & 136 & $12,9 \%$ & 6 & $8,6 \%$ & & \\
\hline \multicolumn{9}{|l|}{$\begin{array}{l}\text { Reittage pro Woche } \\
\text { während der SWS }\end{array}$} \\
\hline $\begin{array}{l}\text { nur gelegentlich oder im } \\
\text { Urlaub }\end{array}$ & 31 & 2,8 & 28 & $2,7 \%$ & 3 & $4,3 \%$ & 0,66 & ns \\
\hline 1x pro Woche & 75 & 6,7 & 71 & $6,7 \%$ & 4 & $5,7 \%$ & 0,36 & ns \\
\hline täglich & 180 & 16,0 & 175 & $16,6 \%$ & 5 & $7,1 \%$ & 2,89 & $\mathrm{~s}$ \\
\hline nicht weitergeritten & 0 & 0,0 & 0 & $0,0 \%$ & 0 & $0,0 \%$ & & \\
\hline $\begin{array}{l}\text { Insges. Während SWS } \\
\text { weitergeritten }\end{array}$ & 1122 & 100,0 & 1052 & $100,0 \%$ & 70 & $100,0 \%$ & & ns \\
\hline \multicolumn{9}{|l|}{$\begin{array}{l}\text { Durchschnittliche } \\
\text { Reitdauer während der } \\
\text { SWS }\end{array}$} \\
\hline $2-3 \mathrm{~h}$ & 174 & 15,5 & 161 & $15,3 \%$ & 13 & $18,6 \%$ & 0,68 & ns \\
\hline $4 \mathrm{~h}$ und mehr & 12 & 1,1 & 11 & $1,0 \%$ & 1 & $1,4 \%$ & 0,26 & ns \\
\hline & & & & & & & & \\
\hline \multicolumn{9}{|l|}{ Reitzeit pro Woche } \\
\hline bis $0,2 \mathrm{~h}$ & 24 & 2,1 & 22 & $2,1 \%$ & 2 & $2,9 \%$ & 0,38 & ns \\
\hline ca. $0,5 \mathrm{~h}$ & 6 & 0,5 & 5 & $0,5 \%$ & 1 & $1,4 \%$ & 0,66 & ns \\
\hline $1 \mathrm{~h}$ & 66 & 5,9 & 62 & $5,9 \%$ & 4 & $5,7 \%$ & 0,06 & ns \\
\hline $2 \mathrm{~h}$ & 247 & 22,0 & 233 & $22,1 \%$ & 14 & $20,0 \%$ & 0,43 & ns \\
\hline $4 \mathrm{~h}$ & 486 & 43,3 & 453 & $43,1 \%$ & 33 & $47,1 \%$ & 0,66 & ns \\
\hline $5 \mathrm{~h}$ & 34 & 3,0 & 31 & $2,9 \%$ & 3 & $4,3 \%$ & 0,54 & ns \\
\hline $7 \mathrm{~h}$ & 114 & 10,2 & 111 & $10,6 \%$ & 3 & $4,3 \%$ & 2,41 & ns \\
\hline $10 \mathrm{~h}$ & 68 & 6,1 & 60 & $5,7 \%$ & 8 & $11,4 \%$ & 1,48 & ns \\
\hline $17,5 \mathrm{~h}$ & 57 & 5,1 & 56 & $5,3 \%$ & 1 & $1,4 \%$ & 2,47 & ns \\
\hline $35 \mathrm{~h}$ & 9 & 0,8 & 8 & $0,8 \%$ & 1 & $1,4 \%$ & 0,46 & ns \\
\hline \multicolumn{9}{|l|}{ Welche Pferde geritten } \\
\hline überwiegend Schulpferde & 16 & 1,4 & 16 & $1,5 \%$ & 0 & $0,0 \%$ & 4,03 & ns \\
\hline $\begin{array}{l}\text { eigenes Pferd / } \\
\text { Beteiligung }\end{array}$ & 919 & 81,9 & 862 & $81,9 \%$ & 57 & $81,4 \%$ & 0,11 & ns \\
\hline $\begin{array}{l}\text { mehrere Privatpferde / } \\
\text { Ausbildungspferde }\end{array}$ & 109 & 9,7 & 99 & $9,4 \%$ & 10 & $14,3 \%$ & 1,14 & ns \\
\hline keine Angabe & 78 & 7,0 & 75 & $7,1 \%$ & 3 & $4,3 \%$ & 1,12 & ns \\
\hline
\end{tabular}


Reiten in der Schwangerschaft - Primiparae

Anhang Tab. A1

Entbindungen ab $37+0$ vs. Entbindungen bis $36+6$

\begin{tabular}{|c|c|c|c|c|c|c|c|c|}
\hline & $\begin{array}{l}\text { Primi- } \\
\text { parae }\end{array}$ & $\begin{array}{l}\text { Pro- } \\
\text { zent }\end{array}$ & $\begin{array}{c}\text { PP ab } \\
37+0\end{array}$ & $\begin{array}{l}\text { Pro- } \\
\text { zent }\end{array}$ & $\begin{array}{c}\text { PP bis } \\
36+6\end{array}$ & Pro-zent & & \\
\hline$n=$ & 1122 & & 1052 & & 70 & & & \\
\hline \multirow[t]{3}{*}{ Definitionen } & & & & & & & Wurzel S & Signifikanz \\
\hline & 520 & $46,3 \%$ & 484 & $46,0 \%$ & 36 & $51,4 \%$ & 0,88 & ns \\
\hline & 248 & $22,1 \%$ & 235 & $22,3 \%$ & 13 & $18,6 \%$ & 0,78 & ns \\
\hline \multicolumn{9}{|l|}{$\iota$} \\
\hline \multicolumn{9}{|l|}{$\begin{array}{l}\text { Reiterliche Disziplinen } \\
\text { während der Sws }\end{array}$} \\
\hline \multicolumn{9}{|l|}{ Dressur } \\
\hline nicht geritten & 294 & 26,2 & 278 & $26,4 \%$ & 16 & $22,9 \%$ & 0,69 & ns \\
\hline leichte Belastung & 260 & 23,2 & 239 & $22,7 \%$ & 21 & $30,0 \%$ & 1,29 & ns \\
\hline mittlere Belastung & 316 & 28,2 & 297 & $28,2 \%$ & 19 & $27,1 \%$ & 0,20 & ns \\
\hline $\begin{array}{l}\text { leistungsorientiertes } \\
\text { Reiten }\end{array}$ & 204 & 18,2 & 196 & $18,6 \%$ & 8 & $11,4 \%$ & 1,81 & ns \\
\hline Spitzensport & 48 & 4,3 & 42 & $4,0 \%$ & 6 & $8,6 \%$ & 1,35 & ns \\
\hline \multicolumn{9}{|l|}{ Springen } \\
\hline nicht geritten & 909 & 81,0 & 850 & $80,8 \%$ & 59 & $84,3 \%$ & 0,77 & ns \\
\hline leichte Belastung & 112 & 10,0 & 106 & $10,1 \%$ & 6 & $8,6 \%$ & 0,43 & ns \\
\hline mittlere Belastung & 57 & 5,1 & 55 & $5,2 \%$ & 2 & $2,9 \%$ & 1,13 & ns \\
\hline $\begin{array}{l}\text { leistungsorientiertes } \\
\text { Reiten }\end{array}$ & 33 & 2,9 & 31 & $2,9 \%$ & 2 & $2,9 \%$ & 0,04 & ns \\
\hline Spitzensport & 11 & 1,0 & 10 & $1,0 \%$ & 1 & $1,4 \%$ & 0,33 & ns \\
\hline \multicolumn{9}{|l|}{ Ausreiten } \\
\hline nicht geritten & 198 & 17,6 & 187 & $17,8 \%$ & 11 & $15,7 \%$ & 0,46 & ns \\
\hline leichte Belastung & 351 & 31,3 & 326 & $31,0 \%$ & 25 & $35,7 \%$ & 0,80 & ns \\
\hline mittlere Belastung & 464 & 41,4 & 436 & $41,4 \%$ & 28 & $40,0 \%$ & 0,24 & ns \\
\hline $\begin{array}{l}\text { leistungsorientiertes } \\
\text { Reiten }\end{array}$ & 92 & 8,2 & 88 & $8,4 \%$ & 4 & $5,7 \%$ & 0,91 & ns \\
\hline Spitzensport & 17 & 1,5 & 15 & $1,4 \%$ & 2 & $2,9 \%$ & 0,71 & ns \\
\hline \multicolumn{9}{|l|}{ Gelände / Vielseitigkeit } \\
\hline nicht geritten & 905 & 80,7 & 845 & $80,3 \%$ & 60 & $85,7 \%$ & 1,24 & ns \\
\hline leichte Belastung & 110 & 9,8 & 105 & $10,0 \%$ & 5 & $7,1 \%$ & 0,88 & ns \\
\hline mittlere Belastung & 86 & 7,7 & 81 & $7,7 \%$ & 5 & $7,1 \%$ & 0,17 & ns \\
\hline $\begin{array}{l}\text { leistungsorientiertes } \\
\text { Reiten }\end{array}$ & 18 & 1,6 & 18 & $1,7 \%$ & 0 & $0,0 \%$ & 4,28 & ns \\
\hline Spitzensport & 3 & 0,3 & 3 & $0,3 \%$ & 0 & $0,0 \%$ & 1,73 & ns \\
\hline \multicolumn{9}{|l|}{ Gangpferde } \\
\hline nicht geritten & 1051 & 93,7 & 987 & $93,8 \%$ & 64 & $91,4 \%$ & 0,70 & ns \\
\hline leichte Belastung & 21 & 1,9 & 18 & $1,7 \%$ & 3 & $4,3 \%$ & 1,05 & ns \\
\hline mittlere Belastung & 30 & 2,7 & 28 & $2,7 \%$ & 2 & $2,9 \%$ & 0,10 & ns \\
\hline $\begin{array}{l}\text { leistungsorientiertes } \\
\text { Reiten }\end{array}$ & 15 & 1,3 & 14 & $1,3 \%$ & 1 & $1,4 \%$ & 0,07 & ns \\
\hline Spitzensport & 5 & 0,4 & 5 & $0,5 \%$ & 0 & $0,0 \%$ & 2,24 & ns \\
\hline \multicolumn{9}{|l|}{ Western } \\
\hline nicht geritten & 993 & 88,5 & 932 & $88,6 \%$ & 61 & $87,1 \%$ & 0,35 & ns \\
\hline leichte Belastung & 52 & 4,6 & 49 & $4,7 \%$ & 3 & $4,3 \%$ & 0,15 & ns \\
\hline mittlere Belastung & 50 & 4,5 & 46 & $4,4 \%$ & 4 & $5,7 \%$ & 0,47 & ns \\
\hline $\begin{array}{l}\text { leistungsorientiertes } \\
\text { Reiten und Spitzensport }\end{array}$ & 27 & 2,4 & 25 & $2,4 \%$ & 2 & $2,9 \%$ & 0,23 & ns \\
\hline
\end{tabular}


Reiten in der Schwangerschaft - Primiparae

Anhang Tab. A1

Entbindungen ab $37+0$ vs. Entbindungen bis $36+6$

\begin{tabular}{|c|c|c|c|c|c|c|c|c|}
\hline & $\begin{array}{l}\text { Primi- } \\
\text { parae }\end{array}$ & $\begin{array}{l}\text { Pro- } \\
\text { zent }\end{array}$ & $\begin{array}{c}\text { PP ab } \\
37+0\end{array}$ & $\begin{array}{l}\text { Pro- } \\
\text { zent }\end{array}$ & $\begin{array}{l}\text { PP bis } \\
36+6\end{array}$ & Pro-zent & & \\
\hline$n=$ & 1122 & & 1052 & & 70 & & & \\
\hline Definitionen & & & & & & & Wurzel S & Signifikanz \\
\hline \multicolumn{9}{|l|}{ Distanzreiten } \\
\hline nicht geritten & 1093 & 97,4 & 1026 & $97,5 \%$ & 67 & $95,7 \%$ & 0,74 & ns \\
\hline leichte Belastung & 14 & 1,2 & 11 & $1,0 \%$ & 3 & $4,3 \%$ & 1,33 & ns \\
\hline mittlere Belastung & 9 & 0,8 & 9 & $0,9 \%$ & 0 & $0,0 \%$ & 3,01 & ns \\
\hline $\begin{array}{l}\text { leistungsorientiertes } \\
\text { Reiten und Spitzensport }\end{array}$ & 6 & 0,5 & 6 & $0,6 \%$ & 0 & $0,0 \%$ & 2,46 & ns \\
\hline \multicolumn{9}{|l|}{ Reiten-Rubriken } \\
\hline $\begin{array}{l}\text { Dressurbetont, leichte } \\
\text { Belastung }\end{array}$ & 363 & 32,4 & 343 & $32,6 \%$ & 20 & $28,6 \%$ & 0,72 & ns \\
\hline $\begin{array}{l}\text { Dressurbetont, } \\
\text { ambitionierte Belastung }\end{array}$ & 218 & 19,4 & 205 & $19,5 \%$ & 13 & $18,6 \%$ & 0,19 & ns \\
\hline $\begin{array}{l}\text { Springbetont, leichte } \\
\text { Belastung }\end{array}$ & 19 & 1,7 & 17 & $1,6 \%$ & 2 & $2,9 \%$ & 0,61 & ns \\
\hline $\begin{array}{l}\text { Springbetont, } \\
\text { ambitionierte Belastung }\end{array}$ & 63 & 5,6 & 60 & $5,7 \%$ & 3 & $4,3 \%$ & 0,56 & ns \\
\hline $\begin{array}{l}\text { Geländebetont, leichte } \\
\text { Belastung }\end{array}$ & 318 & 28,3 & 293 & $27,9 \%$ & 25 & $35,7 \%$ & 1,33 & ns \\
\hline $\begin{array}{l}\text { Geländebetont, } \\
\text { ambitionierte Belastung }\end{array}$ & 73 & 6,5 & 69 & $6,6 \%$ & 4 & $5,7 \%$ & 0,29 & ns \\
\hline $\begin{array}{l}\text { Nicht geritten/keine } \\
\text { Angabe }\end{array}$ & 68 & 6,1 & 65 & $6,2 \%$ & 3 & $4,3 \%$ & 0,75 & ns \\
\hline \multicolumn{9}{|l|}{$\begin{array}{l}\text { Turnierteilnahmen } \\
\text { während der } \\
\text { Schwangerschaft }\end{array}$} \\
\hline ja & 233 & 20,8 & 215 & $20,4 \%$ & 18 & $25,7 \%$ & 0,98 & ns \\
\hline nein & 811 & 72,3 & 762 & $72,4 \%$ & 49 & $70,0 \%$ & 0,43 & ns \\
\hline keine Angabe & 78 & 7,0 & 75 & $7,1 \%$ & 3 & $4,3 \%$ & & ns \\
\hline \multicolumn{9}{|l|}{$\begin{array}{l}\text { Junge Pferde I } \\
\text { Problempferde geritten }\end{array}$} \\
\hline ja & 261 & 23,3 & 248 & $23,6 \%$ & 13 & $18,6 \%$ & 1,04 & ns \\
\hline nein & 781 & 69,6 & 728 & $69,2 \%$ & 53 & $75,7 \%$ & 1,22 & ns \\
\hline keine Angabe & 80 & 7,1 & 76 & $7,2 \%$ & 4 & $5,7 \%$ & & ns \\
\hline \multicolumn{9}{|l|}{$\begin{array}{l}\text { Reitstil während der } \\
\text { Schwangerschaft } \\
\text { geändert }\end{array}$} \\
\hline ja & 746 & 66,5 & 701 & $66,6 \%$ & 45 & $64,3 \%$ & 0,40 & ns \\
\hline nein & 297 & 26,5 & 275 & $26,1 \%$ & 22 & $31,4 \%$ & 0,93 & ns \\
\hline keine Angabe & 79 & 7,0 & 76 & $7,2 \%$ & 3 & $4,3 \%$ & & ns \\
\hline \multicolumn{9}{|l|}{$\begin{array}{l}\text { Reitstil geändert, und } \\
\text { zwar }\end{array}$} \\
\hline $\begin{array}{l}\text { weniger anstrengender } \\
\text { Reitstil }\end{array}$ & 418 & 37,3 & 393 & $37,4 \%$ & 25 & $35,7 \%$ & 0,28 & ns \\
\hline & & & & & & & & \\
\hline & & & & & & & & \\
\hline
\end{tabular}


Reiten in der Schwangerschaft - Primiparae

Anhang Tab. A1

Entbindungen ab $37+0$ vs. Entbindungen bis $36+6$

\begin{tabular}{|c|c|c|c|c|c|c|c|c|}
\hline & $\begin{array}{l}\text { Primi- } \\
\text { parae }\end{array}$ & $\begin{array}{l}\text { Pro- } \\
\text { zent }\end{array}$ & $\begin{array}{c}\text { PP ab } \\
37+0\end{array}$ & $\begin{array}{l}\text { Pro- } \\
\text { zent }\end{array}$ & $\begin{array}{c}\text { PP bis } \\
36+6\end{array}$ & Pro-zent & & \\
\hline$n=$ & 1122 & & 1052 & & 70 & & & \\
\hline Definitionen & & & & & & & Wurzel S & Signifikanz \\
\hline $\begin{array}{l}\text { im Trab kein Aussitzen, } \\
\text { nur Leichttrab }\end{array}$ & 501 & 44,7 & 469 & $44,6 \%$ & 32 & $45,7 \%$ & 0,18 & ns \\
\hline $\begin{array}{l}\text { im Galopp nur leichter } \\
\text { Sitz }\end{array}$ & 254 & 22,6 & 237 & $22,5 \%$ & 17 & $24,3 \%$ & 0,33 & ns \\
\hline $\begin{array}{l}\text { weniger hohe Sprünge, } \\
\text { weniger Tempo beim } \\
\text { Springen }\end{array}$ & 117 & 10,4 & 111 & $10,6 \%$ & 6 & $8,6 \%$ & 0,57 & ns \\
\hline keine Sprünge mehr & 357 & 31,8 & 338 & $32,1 \%$ & 19 & $27,1 \%$ & 0,91 & ns \\
\hline $\begin{array}{l}\text { weniger } \\
\text { Turnierteilnahmen }\end{array}$ & 91 & 8,1 & 84 & $8,0 \%$ & 7 & $10,0 \%$ & 0,55 & ns \\
\hline $\begin{array}{l}\text { keine Turnierteilnahmen } \\
\text { mehr }\end{array}$ & 237 & 21,1 & 229 & $21,8 \%$ & 8 & $11,4 \%$ & 2,58 & $\mathrm{~s}$ \\
\hline vermehrt ruhige Ausritte & 478 & 42,6 & 449 & $42,7 \%$ & 29 & $41,4 \%$ & 0,21 & ns \\
\hline sonstiges & 150 & 13,4 & 142 & $13,5 \%$ & 8 & $11,4 \%$ & 0,52 & ns \\
\hline keine Angabe & 365 & 32,5 & 339 & $32,2 \%$ & 26 & $37,1 \%$ & 0,83 & ns \\
\hline $\begin{array}{l}\text { Insgesamt Reitstil } \\
\text { geändert }\end{array}$ & 757 & 67,5 & 713 & $67,8 \%$ & 44 & $62,9 \%$ & 0,83 & ns \\
\hline \multicolumn{9}{|l|}{ Unfallrisiko } \\
\hline $\begin{array}{l}\text { fühlte mich völlig sicher, } \\
\text { Unfall ausgeschlossen }\end{array}$ & 525 & 46,8 & 484 & $46,0 \%$ & 41 & $58,6 \%$ & 2,06 & $\mathrm{~s}$ \\
\hline $\begin{array}{l}\text { fühlte mich einigermaßen } \\
\text { sicher, Unfallrisiko gering }\end{array}$ & 453 & 40,4 & 431 & $41,0 \%$ & 22 & $31,4 \%$ & 1,66 & ns \\
\hline keine Angabe & 87 & 7,8 & 84 & $8,0 \%$ & 3 & $4,3 \%$ & & \\
\hline \multicolumn{9}{|l|}{$\begin{array}{l}\text { Unfall beim Reiten oder } \\
\text { beim Umgang mit dem } \\
\text { Pferd }\end{array}$} \\
\hline $\begin{array}{l}\text { ja (10 Frauen mit } \\
\text { Mehrfachnennungen) }\end{array}$ & 106 & 9,4 & 100 & $9,5 \%$ & 6 & $8,6 \%$ & 0,27 & ns \\
\hline nein & 938 & 83,6 & 877 & $83,4 \%$ & 61 & $87,1 \%$ & 0,91 & ns \\
\hline $\begin{array}{l}\text { Kein Umgang mit Pferden } \\
\text { während der } \\
\text { Schwangerschaft }\end{array}$ & 78 & 7,0 & 75 & $7,1 \%$ & 3 & $4,3 \%$ & & ns \\
\hline \multicolumn{9}{|l|}{ Unfälle mit dem Pferd } \\
\hline \multicolumn{9}{|l|}{ Stürze vom Pferd } \\
\hline 1 Sturz & 45 & 4,0 & 41 & $3,9 \%$ & 4 & $5,7 \%$ & 0,64 & ns \\
\hline 2 Stürze & 7 & 0,6 & 7 & $0,7 \%$ & 0 & $0,0 \%$ & & \\
\hline 3 und mehr Stürze & 3 & 0,3 & 2 & $0,2 \%$ & 1 & $1,4 \%$ & & \\
\hline keine Angabe & 1067 & 95,1 & 1002 & $95,2 \%$ & 65 & $92,9 \%$ & & \\
\hline \multicolumn{9}{|l|}{ Stürze mit dem Pferd } \\
\hline 1 Sturz & 20 & 1,8 & 20 & $1,9 \%$ & 0 & $0,0 \%$ & 4,52 & ns \\
\hline 2 Stürze & 3 & 0,3 & 3 & $0,3 \%$ & 0 & $0,0 \%$ & & \\
\hline 3 und mehr Stürze & 0 & 0,0 & 0 & $0,0 \%$ & 0 & $0,0 \%$ & & \\
\hline $\begin{array}{l}\text { Unfälle im Umgang mit } \\
\text { dem Pferd }\end{array}$ & & & & & & & & \\
\hline
\end{tabular}


Reiten in der Schwangerschaft - Primiparae

Anhang Tab. A1

Entbindungen ab $37+0$ vs. Entbindungen bis $36+6$

\begin{tabular}{|c|c|c|c|c|c|c|c|c|}
\hline & $\begin{array}{l}\text { Primi- } \\
\text { parae }\end{array}$ & $\begin{array}{l}\text { Pro- } \\
\text { zent }\end{array}$ & $\begin{array}{c}\text { PP ab } \\
37+0\end{array}$ & $\begin{array}{l}\text { Pro- } \\
\text { zent }\end{array}$ & $\begin{array}{l}\text { PP bis } \\
36+6\end{array}$ & Pro-zent & & \\
\hline$n=$ & 1122 & & 1052 & & 70 & & & \\
\hline Definitionen & & & & & & & Wurzel S & Signifikanz \\
\hline 1 Unfall & 35 & 3,1 & 33 & $3,1 \%$ & 2 & $2,9 \%$ & 0,14 & ns \\
\hline 2 Unfälle & 1 & 0,1 & 1 & $0,1 \%$ & 0 & $0,0 \%$ & 1,00 & ns \\
\hline 3 und mehr Unfälle & 0 & 0,0 & 0 & $0,0 \%$ & 0 & $0,0 \%$ & & \\
\hline kein Unfall & 1 & 0,1 & 1 & $0,1 \%$ & 0 & $0,0 \%$ & & \\
\hline \multicolumn{9}{|l|}{$\begin{array}{l}\text { Unfallfolgen (\% } \\
\text { bezogen auf Unfälle) }\end{array}$} \\
\hline $\begin{array}{l}\text { Unfall blieb ohne Folgen } \\
\text { für die Schwangerschaft }\end{array}$ & 105 & 99,1 & 99 & $99,0 \%$ & 6 & $100,0 \%$ & 3,26 & $\mathrm{~s}$ \\
\hline $\begin{array}{l}\text { Wehen nach dem Unfall } \\
\text { ohne Geburtseintritt }\end{array}$ & 2 & 1,9 & 2 & $2,0 \%$ & 0 & $0,0 \%$ & 4,63 & ns \\
\hline Abort nach dem Unfall & 0 & 0,0 & 0 & $0,0 \%$ & 0 & $0,0 \%$ & & ns \\
\hline $\begin{array}{l}\text { Frühgeburt nach dem } \\
\text { Unfall ( } 34 . \text { Wo) }\end{array}$ & 0 & 0,0 & 0 & $0,0 \%$ & 0 & $0,0 \%$ & & ns \\
\hline \multicolumn{9}{|l|}{$\begin{array}{l}\text { Trainingszustand } \\
\text { während der } \\
\text { Schwangerschaft }\end{array}$} \\
\hline ausgezeichnet & 138 & 12,3 & 128 & $12,2 \%$ & 10 & $14,3 \%$ & 0,49 & ns \\
\hline sehr gut & 220 & 19,6 & 200 & $19,0 \%$ & 20 & $28,6 \%$ & 1,73 & ns \\
\hline gut & 391 & 34,8 & 372 & $35,4 \%$ & 19 & $27,1 \%$ & 1,49 & ns \\
\hline mäßig & 260 & 23,2 & 249 & $23,7 \%$ & 11 & $15,7 \%$ & 1,75 & ns \\
\hline nicht besonders & 113 & 10,1 & 103 & $9,8 \%$ & 10 & $14,3 \%$ & 1,05 & ns \\
\hline Ausdauersportarten & 439 & 39,1 & 413 & $39,3 \%$ & 26 & $37,1 \%$ & 0,35 & ns \\
\hline Gymnastische Sportarten & 139 & 12,4 & 128 & $12,2 \%$ & 11 & $15,7 \%$ & 0,79 & ns \\
\hline Spielsportarten & 21 & 1,9 & 21 & $2,0 \%$ & 0 & $0,0 \%$ & 4,63 & ns \\
\hline Kraftbetonte Sportarten & 29 & 2,6 & 27 & $2,6 \%$ & 2 & $2,9 \%$ & 0,14 & ns \\
\hline $\begin{array}{l}\text { Fun- und } \\
\text { Risikosportarten }\end{array}$ & 58 & 5,2 & 56 & $5,3 \%$ & 2 & $2,9 \%$ & 1,17 & ns \\
\hline Insgesamt sportlich aktiv & 595 & 53,0 & 560 & $53,2 \%$ & 35 & $50,0 \%$ & 0,52 & ns \\
\hline Eine Sportart & 494 & 44,0 & 465 & $44,2 \%$ & 29 & $41,4 \%$ & 0,46 & ns \\
\hline zwei Sportarten & 99 & 8,8 & 93 & $8,8 \%$ & 6 & $8,6 \%$ & 0,08 & ns \\
\hline drei Sportarten & 2 & 0,2 & 2 & $0,2 \%$ & 0 & $0,0 \%$ & 1,42 & ns \\
\hline \multicolumn{9}{|l|}{$\begin{array}{l}\text { Erkrankung vor oder } \\
\text { während der } \\
\text { Schwangerschaft }\end{array}$} \\
\hline \multicolumn{9}{|l|}{ Bluthochdruck } \\
\hline nein & 1057 & 94,2 & 997 & $94,8 \%$ & 60 & $85,7 \%$ & 2,14 & $\mathrm{~s}$ \\
\hline vor der Schwangerschaft & 8 & 0,7 & 8 & $0,8 \%$ & 0 & $0,0 \%$ & 2,84 & ns \\
\hline $\begin{array}{l}\text { während der } \\
\text { Schwangerschaft }\end{array}$ & 57 & 5,1 & 47 & $4,5 \%$ & 10 & $14,3 \%$ & 2,32 & $\mathrm{~s}$ \\
\hline keine Angabe & 0 & 0,0 & 0 & $0,0 \%$ & 0 & $0,0 \%$ & & ns \\
\hline \multicolumn{9}{|l|}{ Diabetes mellitus } \\
\hline nein & 1110 & 98,9 & 1043 & $99,1 \%$ & 67 & $95,7 \%$ & 1,41 & ns \\
\hline vor der Schwangerschaft & 3 & 0,3 & 2 & $0,2 \%$ & 1 & $1,4 \%$ & 0,87 & ns \\
\hline
\end{tabular}


Reiten in der Schwangerschaft - Primiparae

Anhang Tab. A1

Entbindungen ab $37+0$ vs. Entbindungen bis $36+6$

\begin{tabular}{|c|c|c|c|c|c|c|c|c|}
\hline & $\begin{array}{l}\text { Primi- } \\
\text { parae }\end{array}$ & $\begin{array}{l}\text { Pro- } \\
\text { zent }\end{array}$ & $\begin{array}{c}\text { PP ab } \\
37+0\end{array}$ & $\begin{array}{l}\text { Pro- } \\
\text { zent }\end{array}$ & $\begin{array}{l}\text { PP bis } \\
36+6\end{array}$ & Pro-zent & & \\
\hline$n=$ & 1122 & & 1052 & & 70 & & & \\
\hline Definitionen & & & & & & & Wurzel S & Signifikanz \\
\hline $\begin{array}{l}\text { während der } \\
\text { Schwangerschaft }\end{array}$ & 9 & 0,8 & 7 & $0,7 \%$ & 2 & $2,9 \%$ & 1,09 & ns \\
\hline \multicolumn{9}{|l|}{2} \\
\hline \multicolumn{9}{|l|}{ Herzkrankheit } \\
\hline nein & 1116 & 99,5 & 1047 & $99,5 \%$ & 69 & $98,6 \%$ & 0,66 & ns \\
\hline vor der Schwangerschaft & 4 & 0,4 & 4 & $0,4 \%$ & 0 & $0,0 \%$ & 2,00 & ns \\
\hline $\begin{array}{l}\text { während der } \\
\text { Schwangerschaft }\end{array}$ & 2 & 0,2 & 1 & $0,1 \%$ & 1 & $1,4 \%$ & 0,94 & ns \\
\hline \multicolumn{9}{|l|}{3} \\
\hline \multicolumn{9}{|l|}{ Asthma } \\
\hline nein & 1078 & 96,1 & 1012 & $96,2 \%$ & 66 & $94,3 \%$ & 0,67 & ns \\
\hline vor der Schwangerschaft & 38 & 3,4 & 35 & $3,3 \%$ & 3 & $4,3 \%$ & 0,39 & ns \\
\hline $\begin{array}{l}\text { während der } \\
\text { Schwangerschaft }\end{array}$ & 6 & 0,5 & 5 & $0,5 \%$ & 1 & $1,4 \%$ & 0,66 & ns \\
\hline \multicolumn{9}{|l|}{4} \\
\hline \multicolumn{9}{|l|}{ Bronchitis } \\
\hline nein & 1066 & 95,0 & 1002 & $95,2 \%$ & 64 & $91,4 \%$ & 1,12 & ns \\
\hline vor der Schwangerschaft & 30 & 2,7 & 25 & $2,4 \%$ & 5 & $7,1 \%$ & 1,53 & ns \\
\hline $\begin{array}{l}\text { während der } \\
\text { Schwangerschaft }\end{array}$ & 26 & 2,3 & 25 & $2,4 \%$ & 1 & $1,4 \%$ & 0,63 & ns \\
\hline \multicolumn{9}{|l|}{5} \\
\hline \multicolumn{9}{|l|}{$\begin{array}{l}\text { Krampfadern, off. Bein, } \\
\text { Venenthrombose }\end{array}$} \\
\hline nein & 1065 & 94,9 & 996 & $94,7 \%$ & 69 & $98,6 \%$ & 2,47 & $s$ \\
\hline vor der Schwangerschaft & 8 & 0,7 & 7 & $0,7 \%$ & 1 & $1,4 \%$ & 0,53 & ns \\
\hline $\begin{array}{l}\text { während der } \\
\text { Schwangerschaft }\end{array}$ & 49 & 4,4 & 49 & $4,7 \%$ & 0 & $0,0 \%$ & 7,17 & ns \\
\hline \multicolumn{9}{|l|}{6} \\
\hline \multicolumn{9}{|l|}{ Hypothyreose } \\
\hline nein & 1084 & 96,6 & 1015 & $96,5 \%$ & 69 & $98,6 \%$ & 1,37 & ns \\
\hline vor der Schwangerschaft & 30 & 2,7 & 29 & $2,8 \%$ & 1 & $1,4 \%$ & 0,88 & ns \\
\hline $\begin{array}{l}\text { während der } \\
\text { Schwangerschaft }\end{array}$ & 8 & 0,7 & 8 & $0,8 \%$ & 0 & $0,0 \%$ & 2,84 & ns \\
\hline \multicolumn{9}{|l|}{7} \\
\hline \multicolumn{9}{|l|}{ Hyperthyreose } \\
\hline nein & 1112 & 99,1 & 1043 & $99,1 \%$ & 69 & $98,6 \%$ & 0,40 & ns \\
\hline vor der Schwangerschaft & 5 & 0,4 & 5 & $0,5 \%$ & 0 & $0,0 \%$ & 2,24 & ns \\
\hline $\begin{array}{l}\text { während der } \\
\text { Schwangerschaft }\end{array}$ & 5 & 0,4 & 4 & $0,4 \%$ & 1 & $1,4 \%$ & 0,73 & ns \\
\hline \multicolumn{9}{|l|}{8} \\
\hline \multicolumn{9}{|l|}{ Rückenschmerzen } \\
\hline nein & 664 & 59,2 & 627 & $59,6 \%$ & 37 & $52,9 \%$ & 1,10 & ns \\
\hline vor der Schwangerschaft & 137 & 12,2 & 128 & $12,2 \%$ & 9 & $12,9 \%$ & 0,17 & ns \\
\hline $\begin{array}{l}\text { während der } \\
\text { Schwangerschaft }\end{array}$ & 321 & 28,6 & 297 & $28,2 \%$ & 24 & $34,3 \%$ & 1,04 & ns \\
\hline & & & & & & & & \\
\hline
\end{tabular}


Reiten in der Schwangerschaft - Primiparae

Anhang Tab. A1

Entbindungen ab $37+0$ vs. Entbindungen bis $36+6$

\begin{tabular}{|c|c|c|c|c|c|c|c|c|}
\hline & $\begin{array}{l}\text { Primi- } \\
\text { parae }\end{array}$ & $\begin{array}{l}\text { Pro- } \\
\text { zent }\end{array}$ & $\begin{array}{c}\text { PP ab } \\
37+0\end{array}$ & $\begin{array}{l}\text { Pro- } \\
\text { zent }\end{array}$ & $\begin{array}{l}\text { PP bis } \\
36+6\end{array}$ & Pro-zent & & \\
\hline$n=$ & 1122 & & 1052 & & 70 & & & \\
\hline Definitionen & & & & & & & Wurzel S & Signifikanz \\
\hline \multirow{2}{*}{\multicolumn{9}{|c|}{9}} \\
\hline & & & & & & & & \\
\hline \multicolumn{9}{|l|}{ Karies } \\
\hline nein & 945 & 84,2 & 889 & $84,5 \%$ & 56 & $80,0 \%$ & 0,92 & ns \\
\hline vor der Schwangerschaft & 123 & 11,0 & 111 & $10,6 \%$ & 12 & $17,1 \%$ & 1,43 & ns \\
\hline $\begin{array}{l}\text { während der } \\
\text { Schwangerschaft }\end{array}$ & 54 & 4,8 & 52 & $4,9 \%$ & 2 & $2,9 \%$ & 0,99 & ns \\
\hline \multicolumn{9}{|l|}{10} \\
\hline \multicolumn{9}{|l|}{ Parodontitis } \\
\hline nein & 836 & 74,5 & 786 & $74,7 \%$ & 50 & $71,4 \%$ & 0,59 & ns \\
\hline vor der Schwangerschaft & 43 & 3,8 & 37 & $3,5 \%$ & 6 & $8,6 \%$ & 1,49 & ns \\
\hline $\begin{array}{l}\text { während der } \\
\text { Schwangerschaft }\end{array}$ & 243 & 21,7 & 229 & $21,8 \%$ & 14 & $20,0 \%$ & 0,36 & ns \\
\hline \multicolumn{9}{|l|}{11} \\
\hline \multicolumn{9}{|l|}{ Unfallfolgen } \\
\hline nein & 1087 & 96,9 & 1023 & $97,2 \%$ & 64 & $91,4 \%$ & 1,72 & ns \\
\hline $\begin{array}{l}\text { Aus einem Unfall vor der } \\
\text { Schwangerschaft }\end{array}$ & 32 & 2,9 & 26 & $2,5 \%$ & 6 & $8,6 \%$ & 1,80 & ns \\
\hline $\begin{array}{l}\text { Aus einem Unfall } \\
\text { während der } \\
\text { Schwangerschaft }\end{array}$ & 18 & 1,6 & 16 & $1,5 \%$ & 2 & $2,9 \%$ & 0,66 & ns \\
\hline \multicolumn{9}{|l|}{12} \\
\hline \multicolumn{9}{|l|}{ Sonstiges } \\
\hline nein & 955 & 85,1 & 897 & $85,3 \%$ & 58 & $82,9 \%$ & 0,52 & ns \\
\hline vor der Schwangerschaft & 40 & 3,6 & 36 & $3,4 \%$ & 4 & $5,7 \%$ & 0,81 & ns \\
\hline $\begin{array}{l}\text { während der } \\
\text { Schwangerschaft }\end{array}$ & 117 & 10,4 & 110 & $10,5 \%$ & 7 & $10,0 \%$ & 0,12 & ns \\
\hline Keine Angabe & 2 & 0,2 & 2 & $0,2 \%$ & 0 & $0,0 \%$ & & ns \\
\hline \multicolumn{9}{|l|}{$\begin{array}{l}\text { Komplikationen } \\
\text { während der } \\
\text { Schwangerschaft }\end{array}$} \\
\hline ja & 559 & 49,8 & 510 & $48,5 \%$ & 49 & $70,0 \%$ & 3,78 & $\mathrm{~s}$ \\
\hline nein & 562 & 50,1 & 541 & $51,4 \%$ & 21 & $30,0 \%$ & 3,77 & $\mathrm{~s}$ \\
\hline \multicolumn{9}{|l|}{$\begin{array}{l}\text { Komplikationen } \\
\text { während der } \\
\text { Schwangerschaft, und } \\
\text { zwar }\end{array}$} \\
\hline \multicolumn{9}{|l|}{$\begin{array}{l}\text { Scheiden- oder } \\
\text { Harnwegsinfekt }\end{array}$} \\
\hline nein & 1029 & 91,7 & 963 & $91,5 \%$ & 66 & $94,3 \%$ & 0,95 & ns \\
\hline $\begin{array}{l}\text { während der aktiven } \\
\text { Reitzeit bis } 1 \text { Woche } \\
\text { danach }\end{array}$ & 61 & 5,4 & 58 & $5,5 \%$ & 3 & $4,3 \%$ & 0,49 & ns \\
\hline später & 32 & 2,9 & 31 & $2,9 \%$ & 1 & $1,4 \%$ & 1,00 & ns \\
\hline \multicolumn{9}{|l|}{1} \\
\hline \multicolumn{9}{|l|}{$\begin{array}{l}\text { Vorzeitige } \\
\text { Wehentätigkeit }\end{array}$} \\
\hline nein & 996 & 88,8 & 943 & $89,6 \%$ & 53 & $75,7 \%$ & 2,67 & $\mathrm{~s}$ \\
\hline $\begin{array}{l}\text { während der aktiven } \\
\text { Reitzeit bis } 1 \text { Woche } \\
\text { danach }\end{array}$ & 38 & 3,4 & 33 & $3,1 \%$ & 5 & $7,1 \%$ & 1,28 & ns \\
\hline
\end{tabular}


Reiten in der Schwangerschaft - Primiparae

Anhang Tab. A1

Entbindungen ab $37+0$ vs. Entbindungen bis $36+6$

\begin{tabular}{|c|c|c|c|c|c|c|c|c|}
\hline & $\begin{array}{l}\text { Primi- } \\
\text { parae }\end{array}$ & $\begin{array}{l}\text { Pro- } \\
\text { zent }\end{array}$ & $\begin{array}{c}\text { PP ab } \\
37+0\end{array}$ & $\begin{array}{l}\text { Pro- } \\
\text { zent }\end{array}$ & $\begin{array}{l}\text { PP bis } \\
36+6\end{array}$ & Pro-zent & & \\
\hline$n=$ & 1122 & & 1052 & & 70 & & & \\
\hline Definitionen & & & & & & & Wurzel S & Signifikanz \\
\hline später & 88 & 7,8 & 76 & $7,2 \%$ & 12 & $17,1 \%$ & 2,17 & $\mathrm{~s}$ \\
\hline \multicolumn{9}{|l|}{2} \\
\hline \multicolumn{9}{|l|}{\begin{tabular}{|l} 
Vorzeitiger \\
Blasensprung
\end{tabular}} \\
\hline nein & 1053 & 93,9 & 1004 & $95,4 \%$ & 49 & $70,0 \%$ & 4,61 & $\mathrm{~s}$ \\
\hline $\begin{array}{l}\text { während der aktiven } \\
\text { Reitzeit bis } 1 \text { Woche } \\
\text { danach }\end{array}$ & 15 & 1,3 & 11 & $1,0 \%$ & 4 & $5,7 \%$ & 1,67 & ns \\
\hline später & 54 & 4,8 & 37 & $3,5 \%$ & 17 & $24,3 \%$ & 4,03 & $s$ \\
\hline \multicolumn{9}{|l|}{3} \\
\hline \multicolumn{9}{|l|}{ Zervixinsuffizienz } \\
\hline nein & 1057 & 94,2 & 999 & $95,0 \%$ & 58 & $82,9 \%$ & 2,66 & $\mathrm{~s}$ \\
\hline $\begin{array}{l}\text { während der aktiven } \\
\text { Reitzeit bis } 1 \text { Woche } \\
\text { danach }\end{array}$ & 26 & 2,3 & 22 & $2,1 \%$ & 4 & $5,7 \%$ & 1,29 & ns \\
\hline später & 39 & 3,5 & 31 & $2,9 \%$ & 8 & $11,4 \%$ & 2,21 & $\mathrm{~s}$ \\
\hline \multicolumn{9}{|l|}{4} \\
\hline \multicolumn{9}{|c|}{$\begin{array}{l}\text { Amnioninfektionssyndr } \\
\text { om }\end{array}$} \\
\hline nein & 1118 & 99,6 & 1050 & $99,8 \%$ & 68 & $97,1 \%$ & 1,34 & ns \\
\hline $\begin{array}{l}\text { während der aktiven } \\
\text { Reitzeit bis } 1 \text { Woche } \\
\text { danach }\end{array}$ & 0 & 0,0 & 0 & $0,0 \%$ & 0 & $0,0 \%$ & & \\
\hline später & 4 & 0,4 & 2 & $0,2 \%$ & 2 & $2,9 \%$ & 1,34 & ns \\
\hline \multicolumn{9}{|l|}{5} \\
\hline \multicolumn{9}{|c|}{ Ablösung der Plazenta } \\
\hline nein & 1117 & 99,6 & 1049 & $99,7 \%$ & 68 & $97,1 \%$ & 1,29 & ns \\
\hline $\begin{array}{l}\text { während der aktiven } \\
\text { Reitzeit bis } 1 \text { Woche } \\
\text { danach }\end{array}$ & 0 & 0,0 & 0 & $0,0 \%$ & 0 & $0,0 \%$ & & \\
\hline später & 5 & 0,4 & 3 & $0,3 \%$ & 2 & $2,9 \%$ & 1,29 & ns \\
\hline \multicolumn{9}{|l|}{6} \\
\hline \multicolumn{9}{|l|}{ Plazenta-Insuffizienz } \\
\hline nein & 1098 & 97,9 & 1036 & $98,5 \%$ & 62 & $88,6 \%$ & 2,59 & $s$ \\
\hline $\begin{array}{l}\text { während der aktiven } \\
\text { Reitzeit bis } 1 \text { Woche } \\
\text { danach }\end{array}$ & 9 & 0,8 & 6 & $0,6 \%$ & 3 & $4,3 \%$ & 1,53 & ns \\
\hline später & 15 & 1,3 & 10 & $1,0 \%$ & 5 & $7,1 \%$ & 2,00 & $\mathrm{~s}$ \\
\hline \multicolumn{9}{|l|}{7} \\
\hline \multicolumn{9}{|l|}{ Bluthochdruck } \\
\hline nein & 1071 & 95,5 & 1010 & $96,0 \%$ & 61 & $87,1 \%$ & 2,19 & $\mathrm{~s}$ \\
\hline $\begin{array}{l}\text { während der aktiven } \\
\text { Reitzeit bis } 1 \text { Woche } \\
\text { danach }\end{array}$ & 14 & 1,2 & 12 & $1,1 \%$ & 2 & $2,9 \%$ & 0,85 & ns \\
\hline später & 37 & 3,3 & 30 & $2,9 \%$ & 7 & $10,0 \%$ & 1,97 & $\mathrm{~s}$ \\
\hline \multicolumn{9}{|l|}{8} \\
\hline \multicolumn{9}{|c|}{ Präeklampsie / Gestose } \\
\hline nein & 1098 & 97,9 & 1032 & $98,1 \%$ & 66 & $94,3 \%$ & 1,36 & ns \\
\hline $\begin{array}{l}\text { während der aktiven } \\
\text { Reitzeit bis } 1 \text { Woche } \\
\text { danach }\end{array}$ & 2 & 0,2 & 2 & $0,2 \%$ & 0 & $0,0 \%$ & 1,42 & ns \\
\hline später & 22 & 2,0 & 18 & $1,7 \%$ & 4 & $5,7 \%$ & 1,43 & ns \\
\hline
\end{tabular}


Reiten in der Schwangerschaft - Primiparae

Anhang Tab. A1

Entbindungen ab $37+0$ vs. Entbindungen bis $36+6$

\begin{tabular}{|c|c|c|c|c|c|c|c|c|}
\hline & $\begin{array}{l}\text { Primi- } \\
\text { parae }\end{array}$ & $\begin{array}{l}\text { Pro- } \\
\text { zent }\end{array}$ & $\begin{array}{c}P P a b \\
37+0\end{array}$ & $\begin{array}{l}\text { Pro- } \\
\text { zent }\end{array}$ & $\begin{array}{c}\text { PP bis } \\
36+6\end{array}$ & Pro-zent & & \\
\hline$n=$ & 1122 & & 1052 & & 70 & & & \\
\hline Definitionen & & & & & & & Wurzel S & Signifikanz \\
\hline \multicolumn{9}{|c|}{ 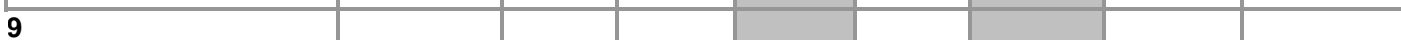 } \\
\hline \multicolumn{9}{|l|}{$\begin{array}{l}\text { Eklampsie I HELLP- } \\
\text { Syndrom }\end{array}$} \\
\hline nein & 1113 & 99,2 & 1045 & $99,3 \%$ & 68 & $97,1 \%$ & 1,09 & ns \\
\hline $\begin{array}{l}\text { während der aktiven } \\
\text { Reitzeit bis } 1 \text { Woche } \\
\text { danach }\end{array}$ & 2 & 0,2 & 2 & $0,2 \%$ & 0 & $0,0 \%$ & 1,42 & ns \\
\hline später & 7 & 0,6 & 5 & $0,5 \%$ & 2 & $2,9 \%$ & 1,19 & ns \\
\hline \multicolumn{9}{|l|}{10} \\
\hline \multicolumn{9}{|l|}{$\begin{array}{l}\text { Schwangerschafts- } \\
\text { diabetes }\end{array}$} \\
\hline nein & 1103 & 98,3 & 1035 & $98,4 \%$ & 68 & $97,1 \%$ & 0,61 & ns \\
\hline $\begin{array}{l}\text { während der aktiven } \\
\text { Reitzeit bis } 1 \text { Woche } \\
\text { danach }\end{array}$ & 5 & 0,4 & 5 & $0,5 \%$ & 0 & $0,0 \%$ & 2,24 & ns \\
\hline später & 14 & 1,2 & 12 & $1,1 \%$ & 2 & $2,9 \%$ & 0,85 & ns \\
\hline \multicolumn{9}{|l|}{11} \\
\hline \multicolumn{9}{|l|}{ Hydramnion } \\
\hline nein & 1117 & 99,6 & 1048 & $99,6 \%$ & 69 & $98,6 \%$ & 0,73 & ns \\
\hline $\begin{array}{l}\text { während der aktiven } \\
\text { Reitzeit bis } 1 \text { Woche } \\
\text { danach }\end{array}$ & 1 & 0,1 & 1 & $0,1 \%$ & 0 & $0,0 \%$ & 1,00 & ns \\
\hline später & 4 & 0,4 & 3 & $0,3 \%$ & 1 & $1,4 \%$ & 0,80 & ns \\
\hline \multicolumn{9}{|l|}{12} \\
\hline \multicolumn{9}{|l|}{ Fehlbildungen } \\
\hline nein & 1120 & 99,8 & 1051 & $99,9 \%$ & 69 & $98,6 \%$ & 0,94 & ns \\
\hline $\begin{array}{l}\text { während der aktiven } \\
\text { Reitzeit bis } 1 \text { Woche } \\
\text { danach }\end{array}$ & 0 & 0,0 & 0 & $0,0 \%$ & 0 & $0,0 \%$ & & \\
\hline später & 2 & 0,2 & 1 & $0,1 \%$ & 1 & $1,4 \%$ & 0,94 & ns \\
\hline \multicolumn{9}{|l|}{13} \\
\hline \multicolumn{9}{|l|}{ Uterusmyom } \\
\hline nein & 1111 & 99,0 & 1041 & $99,0 \%$ & 70 & $100,0 \%$ & 3,33 & $\mathrm{~s}$ \\
\hline $\begin{array}{l}\text { während der aktiven } \\
\text { Reitzeit bis } 1 \text { Woche } \\
\text { danach }\end{array}$ & 3 & 0,3 & 3 & $0,3 \%$ & 0 & $0,0 \%$ & 1,73 & ns \\
\hline später & 8 & 0,7 & 8 & $0,8 \%$ & 0 & $0,0 \%$ & 2,84 & ns \\
\hline \multicolumn{9}{|l|}{14} \\
\hline \multicolumn{9}{|l|}{ Blutungen } \\
\hline nein & 1067 & 95,1 & 1005 & $95,5 \%$ & 62 & $88,6 \%$ & 1,81 & ns \\
\hline $\begin{array}{l}\text { während der aktiven } \\
\text { Reitzeit bis } 1 \text { Woche } \\
\text { danach }\end{array}$ & 46 & 4,1 & 39 & $3,7 \%$ & 7 & $10,0 \%$ & 1,73 & ns \\
\hline später & 9 & 0,8 & 8 & $0,8 \%$ & 1 & $1,4 \%$ & 0,46 & ns \\
\hline \multicolumn{9}{|l|}{15} \\
\hline \multicolumn{9}{|l|}{ Fieber } \\
\hline nein & 1102 & 98,2 & 1033 & $98,2 \%$ & 69 & $98,6 \%$ & 0,26 & ns \\
\hline $\begin{array}{l}\text { während der aktiven } \\
\text { Reitzeit bis } 1 \text { Woche } \\
\text { danach }\end{array}$ & 10 & 0,9 & 10 & $1,0 \%$ & 0 & $0,0 \%$ & 3,18 & ns \\
\hline später & 10 & 0,9 & 9 & $0,9 \%$ & 1 & $1,4 \%$ & 0,40 & ns \\
\hline & & & & & & & & \\
\hline & & & & & & & & \\
\hline & & & & & & & & \\
\hline
\end{tabular}


Reiten in der Schwangerschaft - Primiparae

Anhang Tab. A1

Entbindungen ab $37+0$ vs. Entbindungen bis $36+6$

\begin{tabular}{|c|c|c|c|c|c|c|c|c|}
\hline & $\begin{array}{l}\text { Primi- } \\
\text { parae }\end{array}$ & $\begin{array}{l}\text { Pro- } \\
\text { zent }\end{array}$ & $\begin{array}{c}\text { PP ab } \\
37+0\end{array}$ & $\begin{array}{l}\text { Pro- } \\
\text { zent }\end{array}$ & $\begin{array}{l}\text { PP bis } \\
36+6\end{array}$ & Pro-zent & & \\
\hline$n=$ & 1122 & & 1052 & & 70 & & & \\
\hline Definitionen & & & & & & & Wurzel S & Signifikanz \\
\hline \multicolumn{9}{|l|}{16} \\
\hline \multicolumn{9}{|l|}{$\begin{array}{l}\text { Blutgruppen- } \\
\text { Unverträglichkeit }\end{array}$} \\
\hline nein & 1108 & 98,8 & 1038 & $98,7 \%$ & 70 & $100,0 \%$ & 3,77 & $\mathbf{s}$ \\
\hline $\begin{array}{l}\text { während der aktiven } \\
\text { Reitzeit bis } 1 \text { Woche } \\
\text { danach }\end{array}$ & 7 & 0,6 & 7 & $0,7 \%$ & 0 & $0,0 \%$ & 2,65 & $\mathrm{~ns}$ \\
\hline später & 7 & 0,6 & 7 & $0,7 \%$ & 0 & $0,0 \%$ & 2,65 & ns \\
\hline \multicolumn{9}{|l|}{17} \\
\hline \multicolumn{9}{|l|}{ Toxoplasmose } \\
\hline nein & 1120 & 99,8 & 1050 & $99,8 \%$ & 70 & $100,0 \%$ & 1,42 & ns \\
\hline $\begin{array}{l}\text { während der aktiven } \\
\text { Reitzeit bis } 1 \text { Woche } \\
\text { danach }\end{array}$ & 2 & 0,2 & 2 & $0,2 \%$ & 0 & $0,0 \%$ & 1,42 & ns \\
\hline später & 0 & 0,0 & 0 & $0,0 \%$ & 0 & $0,0 \%$ & & \\
\hline \multicolumn{9}{|l|}{18} \\
\hline \multicolumn{9}{|l|}{$\begin{array}{l}\text { Sonstige } \\
\text { Infektionskrankheiten }\end{array}$} \\
\hline nein & 1090 & 97,1 & 1022 & $97,1 \%$ & 68 & $97,1 \%$ & 0,00 & ns \\
\hline $\begin{array}{l}\text { während der aktiven } \\
\text { Reitzeit bis } 1 \text { Woche } \\
\text { danach }\end{array}$ & 16 & 1,4 & 16 & $1,5 \%$ & 0 & $0,0 \%$ & 4,03 & ns \\
\hline später & 16 & 1,4 & 14 & $1,3 \%$ & 2 & $2,9 \%$ & 0,75 & ns \\
\hline \multicolumn{9}{|l|}{19} \\
\hline \multicolumn{9}{|l|}{$\begin{array}{l}\text { Sonstige schwere } \\
\text { Krankheiten }\end{array}$} \\
\hline nein & 1120 & 99,8 & 1050 & $99,8 \%$ & 70 & $100,0 \%$ & 1,42 & ns \\
\hline $\begin{array}{l}\text { während der aktiven } \\
\text { Reitzeit bis } 1 \text { Woche } \\
\text { danach }\end{array}$ & 1 & 0,1 & 1 & $0,1 \%$ & 0 & $0,0 \%$ & 1,00 & ns \\
\hline später & 1 & 0,1 & 1 & $0,1 \%$ & 0 & $0,0 \%$ & 1,00 & ns \\
\hline \multicolumn{9}{|l|}{20} \\
\hline \multicolumn{9}{|l|}{$\begin{array}{l}\text { Falsche Lage des } \\
\text { Kindes }\end{array}$} \\
\hline nein & 1061 & 94,6 & 999 & $95,0 \%$ & 62 & $88,6 \%$ & 1,65 & ns \\
\hline $\begin{array}{l}\text { während der aktiven } \\
\text { Reitzeit bis } 1 \text { Woche } \\
\text { danach }\end{array}$ & 25 & 2,2 & 22 & $2,1 \%$ & 3 & $4,3 \%$ & 0,89 & ns \\
\hline später & 36 & 3,2 & 31 & $2,9 \%$ & 5 & $7,1 \%$ & 1,34 & ns \\
\hline \multicolumn{9}{|l|}{21} \\
\hline \multicolumn{9}{|l|}{ Sonstiges } \\
\hline nein & 1031 & 91,9 & 967 & $91,9 \%$ & 64 & $91,4 \%$ & 0,14 & ns \\
\hline $\begin{array}{l}\text { während der aktiven } \\
\text { Reitzeit bis } 1 \text { Woche } \\
\text { danach }\end{array}$ & 49 & 4,4 & 47 & $4,5 \%$ & 2 & $2,9 \%$ & 0,77 & ns \\
\hline später & 42 & 3,7 & 38 & $3,6 \%$ & 4 & $5,7 \%$ & 0,74 & ns \\
\hline \multicolumn{9}{|l|}{22} \\
\hline \multicolumn{9}{|l|}{ Krankenhausaufenthalt } \\
\hline $\begin{array}{l}\text { keine Komplik., kein } \\
\text { Krankenhaus }\end{array}$ & & 47,2 & & $49 \%$ & & $27 \%$ & 3,87 & ns \\
\hline $\begin{array}{l}\text { Komplikationen mit oder } \\
\text { ohne Krankenhaus }\end{array}$ & 1714 & 52,8 & 1593 & $51 \%$ & 121 & $73 \%$ & 3,87 & $\mathrm{~s}$ \\
\hline $\begin{array}{l}\text { Komplikationen, aber } \\
\text { ohne Krankenhaus }\end{array}$ & 434 & 38,7 & 420 & $39,9 \%$ & 14 & $20,0 \%$ & 3,97 & $\mathrm{~s}$ \\
\hline
\end{tabular}


Reiten in der Schwangerschaft - Primiparae

Anhang Tab. A1

Entbindungen ab $37+0$ vs. Entbindungen bis $36+6$

\begin{tabular}{|c|c|c|c|c|c|c|c|c|}
\hline & $\begin{array}{l}\text { Primi- } \\
\text { parae }\end{array}$ & $\begin{array}{l}\text { Pro- } \\
\text { zent }\end{array}$ & $\begin{array}{c}\text { PP ab } \\
37+0\end{array}$ & $\begin{array}{l}\text { Pro- } \\
\text { zent }\end{array}$ & $\begin{array}{l}\text { PP bis } \\
36+6\end{array}$ & Pro-zent & & \\
\hline$n=$ & 1122 & & 1052 & & 70 & & & \\
\hline Definitionen & & & & & & & Wurzel S & Signifikanz \\
\hline $\begin{array}{l}\text { Krankenhausaufenthalt } \\
\text { wg. Komplikationen }\end{array}$ & 158 & 14,1 & 121 & $11,5 \%$ & 37 & $52,9 \%$ & 6,84 & $\mathrm{~s}$ \\
\hline bis 12. Woche & 11 & 1,0 & 8 & $0,8 \%$ & 3 & $4,3 \%$ & 1,45 & ns \\
\hline 13.-20. Woche & 21 & 1,9 & 20 & $1,9 \%$ & 1 & $1,4 \%$ & 0,32 & ns \\
\hline 21.-28. Woche & 18 & 1,6 & 12 & $1,1 \%$ & 6 & $8,6 \%$ & 2,21 & $\mathrm{~s}$ \\
\hline 29.-32. Woche & 31 & 2,8 & 20 & $1,9 \%$ & 11 & $15,7 \%$ & 3,16 & $\mathrm{~s}$ \\
\hline 33.-36. Woche & 39 & 3,5 & 23 & $2,2 \%$ & 16 & $22,9 \%$ & 4,10 & $\mathrm{~s}$ \\
\hline nach 36. Woche & 37 & 3,3 & 37 & $3,5 \%$ & 0 & $0,0 \%$ & 6,19 & ns \\
\hline \multicolumn{9}{|l|}{$\begin{array}{l}\text { Dauer des Kranken- } \\
\text { hausaufenthalts }\end{array}$} \\
\hline bis 7 Tage & 98 & 8,7 & 78 & $7,4 \%$ & 20 & $28,6 \%$ & 3,88 & $\mathrm{~s}$ \\
\hline 8-14 Tage & 38 & 3,4 & 30 & $2,9 \%$ & 8 & $11,4 \%$ & 2,24 & $s$ \\
\hline 15-21 Tage & 9 & 0,8 & 6 & $0,6 \%$ & 3 & $4,3 \%$ & 1,53 & ns \\
\hline 22-28 Tage & 1 & 0,1 & 0 & $0,0 \%$ & 1 & $1,4 \%$ & 1,01 & ns \\
\hline 29-35 Tage & 5 & 0,4 & 1 & $0,1 \%$ & 4 & $5,7 \%$ & 2,02 & ns \\
\hline \multicolumn{9}{|l|}{ über 35 Tage } \\
\hline \multicolumn{9}{|l|}{$\begin{array}{l}\text { Therapien während d. } \\
\text { Schwangerschaft }\end{array}$} \\
\hline \multicolumn{9}{|l|}{ Bettruhe } \\
\hline ja & 149 & 13,3 & 125 & $11,9 \%$ & 24 & $34,3 \%$ & & ns \\
\hline nein & 393 & 35,0 & 367 & $34,9 \%$ & 26 & $37,1 \%$ & & ns \\
\hline keine Eingabe & 580 & 51,7 & 560 & $53,2 \%$ & 20 & $28,6 \%$ & & ns \\
\hline \multicolumn{9}{|l|}{1} \\
\hline \multicolumn{9}{|l|}{ Antibiotika } \\
\hline ja & 58 & 5,2 & 51 & $4,8 \%$ & 7 & $10,0 \%$ & & ns \\
\hline nein & 482 & 43,0 & 440 & $41,8 \%$ & 42 & $60,0 \%$ & & ns \\
\hline keine Eingabe & 582 & 51,9 & 561 & $53,3 \%$ & 21 & $30,0 \%$ & & ns \\
\hline \multicolumn{9}{|l|}{2} \\
\hline \multicolumn{9}{|l|}{ Antimykotika } \\
\hline ja & 58 & 5,2 & 51 & $4,8 \%$ & 7 & $10,0 \%$ & & ns \\
\hline nein & 480 & 42,8 & 438 & $41,6 \%$ & 42 & $60,0 \%$ & & ns \\
\hline keine Eingabe & 584 & 52,0 & 563 & $53,5 \%$ & 21 & $30,0 \%$ & & ns \\
\hline \multicolumn{9}{|l|}{3} \\
\hline \multicolumn{9}{|l|}{ Wehenhemmung } \\
\hline ja & 63 & 5,6 & 51 & $4,8 \%$ & 12 & $17,1 \%$ & & ns \\
\hline nein & 474 & 42,2 & 437 & $41,5 \%$ & 37 & $52,9 \%$ & & ns \\
\hline keine Eingabe & 585 & 52,1 & 564 & $53,6 \%$ & 21 & $30,0 \%$ & & ns \\
\hline \multicolumn{9}{|l|}{4} \\
\hline \multicolumn{9}{|l|}{ Magnesium } \\
\hline ja & 164 & 14,6 & 145 & $13,8 \%$ & 19 & $27,1 \%$ & & ns \\
\hline nein & 377 & 33,6 & 346 & $32,9 \%$ & 31 & $44,3 \%$ & & ns \\
\hline keine Eingabe & 581 & 51,8 & 561 & $53,3 \%$ & 20 & $28,6 \%$ & & ns \\
\hline \multicolumn{9}{|l|}{5} \\
\hline \multicolumn{9}{|l|}{$\begin{array}{l}\text { Medikamente zur } \\
\text { Lungenreifung }\end{array}$} \\
\hline ja & 44 & 3,9 & 27 & $2,6 \%$ & 17 & $24,3 \%$ & & ns \\
\hline nein & 494 & 44,0 & 461 & $43,8 \%$ & 33 & $47,1 \%$ & & ns \\
\hline keine Eingabe & 584 & 52,0 & 564 & $53,6 \%$ & 20 & $28,6 \%$ & & ns \\
\hline \multicolumn{9}{|l|}{6} \\
\hline \multicolumn{9}{|l|}{ Blutdrucksenkung } \\
\hline ja & 25 & 2,2 & 17 & $1,6 \%$ & 8 & $11,4 \%$ & & ns \\
\hline nein & 512 & 45,6 & 470 & $44,7 \%$ & 42 & $60,0 \%$ & & ns \\
\hline keine Eingabe & 585 & 52,1 & 565 & $53,7 \%$ & 20 & $28,6 \%$ & & ns \\
\hline
\end{tabular}


Reiten in der Schwangerschaft - Primiparae

Anhang Tab. A1

Entbindungen ab $37+0$ vs. Entbindungen bis $36+6$

\begin{tabular}{|c|c|c|c|c|c|c|c|c|}
\hline & $\begin{array}{l}\text { Primi- } \\
\text { parae }\end{array}$ & $\begin{array}{l}\text { Pro- } \\
\text { zent }\end{array}$ & $\begin{array}{c}\mathrm{PP} a b \\
37+0\end{array}$ & $\begin{array}{l}\text { Pro- } \\
\text { zent }\end{array}$ & $\begin{array}{c}\text { PP bis } \\
36+6\end{array}$ & Pro-zent & & \\
\hline$n=$ & 1122 & & 1052 & & 70 & & & \\
\hline Definitionen & & & & & & & Wurzel S & Signifikanz \\
\hline \multicolumn{9}{|l|}{7} \\
\hline \multicolumn{9}{|l|}{\begin{tabular}{|l} 
Behandlung des \\
Diabetes
\end{tabular}} \\
\hline ja & 12 & 1,1 & 8 & $0,8 \%$ & 4 & $5,7 \%$ & & ns \\
\hline nein & 525 & 46,8 & 480 & $45,6 \%$ & 45 & $64,3 \%$ & & ns \\
\hline keine Eingabe & 585 & 52,1 & 564 & $53,6 \%$ & 21 & $30,0 \%$ & & ns \\
\hline \multicolumn{9}{|l|}{8} \\
\hline \multicolumn{9}{|l|}{ Cerclage } \\
\hline ja & 11 & 1,0 & 9 & $0,9 \%$ & 2 & $2,9 \%$ & & ns \\
\hline nein & 524 & 46,7 & 478 & $45,4 \%$ & 46 & $65,7 \%$ & & ns \\
\hline keine Eingabe & 587 & 52,3 & 565 & $53,7 \%$ & 22 & $31,4 \%$ & & ns \\
\hline \multicolumn{9}{|l|}{9} \\
\hline \multicolumn{9}{|l|}{ Sonstige Maßnahme } \\
\hline ja & 1002 & 89,3 & 948 & $90,1 \%$ & 54 & $77,1 \%$ & & ns \\
\hline nein & 8 & 0,7 & 8 & $0,8 \%$ & 0 & $0,0 \%$ & & ns \\
\hline keine Eingabe & 112 & 10,0 & 96 & $9,1 \%$ & 16 & $22,9 \%$ & & ns \\
\hline 10 & & & & & & & & ns \\
\hline \multicolumn{9}{|l|}{$\begin{array}{l}\text { Antibiotika /Antimy- } \\
\text { kotika in den } 12 \text { Mo- } \\
\text { naten vor der Sws }\end{array}$} \\
\hline ja & 174 & 15,5 & 160 & $15,2 \%$ & 14 & $20,0 \%$ & 0,98 & ns \\
\hline nein & 947 & 84,4 & 891 & $84,7 \%$ & 56 & $80,0 \%$ & 0,96 & ns \\
\hline \multicolumn{9}{|l|}{ Arbeitsbedingungen } \\
\hline \multicolumn{9}{|l|}{ Schichtarbeit } \\
\hline kam nicht vor & 937 & 83,5 & 877 & $83,4 \%$ & 60 & $85,7 \%$ & & ns \\
\hline kam vor & 184 & 16,4 & 174 & $16,5 \%$ & 10 & $14,3 \%$ & & ns \\
\hline keine Eingabe & 3 & 0,3 & 3 & $0,3 \%$ & 2 & $2,9 \%$ & & ns \\
\hline $\begin{array}{l}\text { Ich fühlte mich dadurch } \\
\text { nicht belastet. }\end{array}$ & 96 & 8,6 & 91 & $8,7 \%$ & 5 & $7,1 \%$ & & ns \\
\hline $\begin{array}{l}\text { Ich fühlte mich dadurch } \\
\text { etwas belastet. }\end{array}$ & 65 & 5,8 & 61 & $5,8 \%$ & 4 & $5,7 \%$ & & ns \\
\hline $\begin{array}{l}\text { Ich fühlte mich dadurch } \\
\text { stark belastet. }\end{array}$ & 21 & 1,9 & 20 & $1,9 \%$ & 1 & $1,4 \%$ & & ns \\
\hline keine Eingabe & 940 & 83,8 & 880 & $83,7 \%$ & 60 & $85,7 \%$ & & ns \\
\hline \multicolumn{9}{|l|}{$\begin{array}{l}\text { Körperlich schwere } \\
\text { Arbeit }\end{array}$} \\
\hline kam nicht vor & 737 & 65,7 & 694 & $66,0 \%$ & 43 & $61,4 \%$ & & ns \\
\hline kam vor & 384 & 34,2 & 357 & $33,9 \%$ & 27 & $38,6 \%$ & & ns \\
\hline keine Eingabe & 1 & 0,1 & 1 & $0,1 \%$ & 0 & $0,0 \%$ & & ns \\
\hline $\begin{array}{l}\text { Ich fühlte mich dadurch } \\
\text { nicht belastet. }\end{array}$ & 141 & 12,6 & 130 & $12,4 \%$ & 11 & $15,7 \%$ & & ns \\
\hline $\begin{array}{l}\text { Ich fühlte mich dadurch } \\
\text { etwas belastet. }\end{array}$ & 202 & 18,0 & 189 & $18,0 \%$ & 13 & $18,6 \%$ & & ns \\
\hline $\begin{array}{l}\text { Ich fühlte mich dadurch } \\
\text { stark belastet. }\end{array}$ & 38 & 3,4 & 35 & $3,3 \%$ & 3 & $4,3 \%$ & & ns \\
\hline keine Eingabe & 741 & 66,0 & 698 & $66,3 \%$ & 43 & $61,4 \%$ & & ns \\
\hline \multicolumn{9}{|l|}{ Starke Konzentration } \\
\hline kam nicht vor & 254 & 22,6 & 234 & $22,2 \%$ & 20 & $28,6 \%$ & & ns \\
\hline kam vor & 867 & 77,3 & 817 & $77,7 \%$ & 50 & $71,4 \%$ & & ns \\
\hline keine Eingabe & 1 & 0,1 & 1 & $0,1 \%$ & 0 & $0,0 \%$ & & ns \\
\hline $\begin{array}{l}\text { Ich fühlte mich dadurch } \\
\text { nicht belastet. }\end{array}$ & 458 & 40,8 & 433 & $41,2 \%$ & 25 & $35,7 \%$ & & ns \\
\hline $\begin{array}{l}\text { Ich fühlte mich dadurch } \\
\text { etwas belastet. }\end{array}$ & 338 & 30,1 & 316 & $30,0 \%$ & 22 & $31,4 \%$ & & ns \\
\hline
\end{tabular}


Reiten in der Schwangerschaft - Primiparae

Anhang Tab. A1

Entbindungen ab $37+0$ vs. Entbindungen bis $36+6$

\begin{tabular}{|c|c|c|c|c|c|c|c|c|}
\hline & $\begin{array}{l}\text { Primi- } \\
\text { parae }\end{array}$ & $\begin{array}{l}\text { Pro- } \\
\text { zent }\end{array}$ & $\begin{array}{c}\mathrm{PP} a b \\
37+0\end{array}$ & $\begin{array}{l}\text { Pro- } \\
\text { zent }\end{array}$ & $\begin{array}{l}\text { PP bis } \\
36+6\end{array}$ & Pro-zent & & \\
\hline$n=$ & 1122 & & 1052 & & 70 & & & \\
\hline Definitionen & & & & & & & Wurzel S & Signifikanz \\
\hline $\begin{array}{l}\text { Ich fühlte mich dadurch } \\
\text { stark belastet. }\end{array}$ & 60 & 5,3 & 57 & $5,4 \%$ & 3 & $4,3 \%$ & & ns \\
\hline keine Eingabe & 266 & 23,7 & 246 & $23,4 \%$ & 20 & $28,6 \%$ & & ns \\
\hline \multicolumn{9}{|l|}{$\begin{array}{l}\text { Zwang zu schnellen } \\
\text { Entscheidungen }\end{array}$} \\
\hline kam nicht vor & 432 & 38,5 & 405 & $38,5 \%$ & 27 & $38,6 \%$ & & ns \\
\hline kam vor & 690 & 61,5 & 647 & $61,5 \%$ & 43 & $61,4 \%$ & & ns \\
\hline keine Eingabe & 0 & 0,0 & 0 & $0,0 \%$ & 0 & $0,0 \%$ & & ns \\
\hline $\begin{array}{l}\text { Ich fühlte mich dadurch } \\
\text { nicht belastet. }\end{array}$ & 393 & 35,0 & 373 & $35,5 \%$ & 20 & $28,6 \%$ & & ns \\
\hline $\begin{array}{l}\text { Ich fühlte mich dadurch } \\
\text { etwas belastet. }\end{array}$ & 239 & 21,3 & 220 & $20,9 \%$ & 19 & $27,1 \%$ & & ns \\
\hline $\begin{array}{l}\text { Ich fühlte mich dadurch } \\
\text { stark belastet. }\end{array}$ & 51 & 4,5 & 48 & $4,6 \%$ & 3 & $4,3 \%$ & & ns \\
\hline keine Eingabe & 439 & 39,1 & 411 & $39,1 \%$ & 28 & $40,0 \%$ & & ns \\
\hline \multicolumn{9}{|l|}{ Arbeit mit Chemikalien } \\
\hline kam nicht vor & 953 & 84,9 & 894 & $85,0 \%$ & 59 & $84,3 \%$ & & ns \\
\hline kam vor & 169 & 15,1 & 158 & $15,0 \%$ & 11 & $15,7 \%$ & & ns \\
\hline keine Eingabe & 0 & 0,0 & 0 & $0,0 \%$ & 0 & $0,0 \%$ & & ns \\
\hline $\begin{array}{l}\text { Ich fühlte mich dadurch } \\
\text { nicht belastet. }\end{array}$ & 109 & 9,7 & 99 & $9,4 \%$ & 10 & $14,3 \%$ & & ns \\
\hline $\begin{array}{l}\text { Ich fühlte mich dadurch } \\
\text { etwas belastet. }\end{array}$ & 40 & 3,6 & 39 & $3,7 \%$ & 1 & $1,4 \%$ & & ns \\
\hline $\begin{array}{l}\text { Ich fühlte mich dadurch } \\
\text { stark belastet. }\end{array}$ & 17 & 1,5 & 17 & $1,6 \%$ & 0 & $0,0 \%$ & & ns \\
\hline keine Eingabe & 956 & 85,2 & 897 & $85,3 \%$ & 59 & $84,3 \%$ & & ns \\
\hline \multicolumn{9}{|l|}{$\begin{array}{l}\text { Ich fühlte mich durch } \\
\text { Arbeit, Beruf, Familie } \\
\text { oder anderes in der } \\
\text { Schwangerschaft } \\
\text { belastet: }\end{array}$} \\
\hline \multicolumn{9}{|l|}{ Häufigkeit } \\
\hline sehr häufig & 70 & 6,2 & 60 & $5,7 \%$ & 10 & $14,3 \%$ & 2,02 & $\mathrm{~s}$ \\
\hline häufig & 177 & 15,8 & 160 & $15,2 \%$ & 17 & $24,3 \%$ & 1,73 & ns \\
\hline gelegentlich & 469 & 41,8 & 446 & $42,4 \%$ & 23 & $32,9 \%$ & 1,64 & ns \\
\hline selten & 360 & 32,1 & 345 & $32,8 \%$ & 15 & $21,4 \%$ & 2,22 & $\mathrm{~s}$ \\
\hline nie & 46 & 4,1 & 41 & $3,9 \%$ & 5 & $7,1 \%$ & 1,04 & ns \\
\hline \multicolumn{9}{|l|}{ Stärke } \\
\hline sehr stark & 65 & 5,8 & 57 & $5,4 \%$ & 8 & $11,4 \%$ & 1,55 & ns \\
\hline stark & 177 & 15,8 & 164 & $15,6 \%$ & 13 & $18,6 \%$ & 0,62 & ns \\
\hline es ging so & 517 & 46,1 & 489 & $46,5 \%$ & 28 & $40,0 \%$ & 1,07 & ns \\
\hline kaum & 315 & 28,1 & 299 & $28,4 \%$ & 16 & $22,9 \%$ & 1,07 & ns \\
\hline nie & 48 & 4,3 & 43 & $4,1 \%$ & 5 & $7,1 \%$ & 0,97 & ns \\
\hline \multicolumn{9}{|l|}{ Koffein } \\
\hline ja & 411 & 36,6 & 382 & $36,3 \%$ & 29 & $41,4 \%$ & 0,84 & ns \\
\hline weniger als 1 Tasse & 129 & 11,5 & 116 & $11,0 \%$ & 13 & $18,6 \%$ & 1,59 & ns \\
\hline $\begin{array}{l}\text { habe fast nie Kaffee } \\
\text { getrunken }\end{array}$ & 581 & 51,8 & 553 & $52,6 \%$ & 28 & $40,0 \%$ & 2,08 & $\mathrm{~s}$ \\
\hline ja, weniger als 2 Tassen & 128 & 11,4 & 122 & $11,6 \%$ & 6 & $8,6 \%$ & & ns \\
\hline ja, weniger als 4 Tassen & 224 & 20,0 & 205 & $19,5 \%$ & 19 & $27,1 \%$ & & \\
\hline
\end{tabular}


Reiten in der Schwangerschaft - Primiparae

Anhang Tab. A1

Entbindungen ab $37+0$ vs. Entbindungen bis $36+6$

\begin{tabular}{|c|c|c|c|c|c|c|c|c|}
\hline & $\begin{array}{l}\text { Primi- } \\
\text { parae }\end{array}$ & $\begin{array}{l}\text { Pro- } \\
\text { zent }\end{array}$ & $\begin{array}{c}\text { PP ab } \\
37+0\end{array}$ & $\begin{array}{l}\text { Pro- } \\
\text { zent }\end{array}$ & $\begin{array}{c}\text { PP bis } \\
36+6\end{array}$ & Pro-zent & & \\
\hline$n=$ & 1122 & & 1052 & & 70 & & & \\
\hline Definitionen & & & & & & & Wurzel S & Signifikanz \\
\hline ja, weniger als 6 Tassen & 22 & 2,0 & 21 & $2,0 \%$ & 1 & $1,4 \%$ & & ns \\
\hline ja, 6 Tassen und mehr & 2 & 0,2 & 1 & $0,1 \%$ & 1 & $1,4 \%$ & & ns \\
\hline \multicolumn{9}{|l|}{ Nikotin } \\
\hline noch nie geraucht & 671 & 59,8 & 635 & $60,4 \%$ & 36 & $51,4 \%$ & 1,45 & ns \\
\hline $\begin{array}{l}\text { vor der Sws schon länger } \\
\text { nicht geraucht }\end{array}$ & 162 & 14,4 & 149 & $14,2 \%$ & 13 & $18,6 \%$ & 0,92 & ns \\
\hline $\begin{array}{l}\text { Rauchen eingestellt nach } \\
\text { Feststellung der Sws }\end{array}$ & 184 & 16,4 & 173 & $16,4 \%$ & 11 & $15,7 \%$ & 0,16 & ns \\
\hline $\begin{array}{l}\text { während der Sws } \\
\text { geraucht }\end{array}$ & 105 & 9,4 & 95 & $9,0 \%$ & 10 & $14,3 \%$ & 1,23 & ns \\
\hline \multicolumn{9}{|l|}{$\begin{array}{l}\text { Zahl der Zigaretten pro } \\
\text { Tag vor der Sws }\end{array}$} \\
\hline keine Zigaretten & 944 & 84,1 & 885 & $84,1 \%$ & 59 & $84,3 \%$ & & ns \\
\hline 1-2 Zigaretten & 13 & 1,2 & 10 & $1,0 \%$ & 3 & $4,3 \%$ & & ns \\
\hline 3-4 Zigaretten & 20 & 1,8 & 19 & $1,8 \%$ & 1 & $1,4 \%$ & & ns \\
\hline 5-6 Zigaretten & 32 & 2,9 & 31 & $2,9 \%$ & 1 & $1,4 \%$ & & ns \\
\hline 7-8 Zigaretten & 5 & 0,4 & 5 & $0,5 \%$ & 0 & $0,0 \%$ & & ns \\
\hline 9-10 Zigaretten & 26 & 2,3 & 24 & $2,3 \%$ & 2 & $2,9 \%$ & & ns \\
\hline mehr als 10 Zigaretten & 82 & 7,3 & 78 & $7,4 \%$ & 4 & $5,7 \%$ & & ns \\
\hline \multicolumn{9}{|l|}{$\begin{array}{l}\text { Zahl der Zigaretten pro } \\
\text { Tag während der Sws }\end{array}$} \\
\hline keine Zigaretteen & 1018 & 90,7 & 958 & $91,1 \%$ & 60 & $85,7 \%$ & 1,25 & ns \\
\hline 1-2 Zigaretten & 24 & 2,1 & 20 & $1,9 \%$ & 4 & $5,7 \%$ & 1,36 & ns \\
\hline 3-4 Zigaretten & 20 & 1,8 & 18 & $1,7 \%$ & 2 & $2,9 \%$ & & ns \\
\hline 5-6 Zigaretten & 30 & 2,7 & 27 & $2,6 \%$ & 3 & $4,3 \%$ & & ns \\
\hline 7-8 Zigaretten & 5 & 0,4 & 5 & $0,5 \%$ & 0 & $0,0 \%$ & & ns \\
\hline 9-10 Zigaretten & 16 & 1,4 & 16 & $1,5 \%$ & 0 & $0,0 \%$ & & ns \\
\hline mehr als 10 Zigaretten & 9 & 0,8 & 8 & $0,8 \%$ & 1 & $1,4 \%$ & & ns \\
\hline \multicolumn{9}{|l|}{ Passivrauchen } \\
\hline ja, bei der Arbeit & 155 & 13,8 & 147 & $14,0 \%$ & 8 & $11,4 \%$ & 0,64 & ns \\
\hline ja, zu Hause & 95 & 8,5 & 86 & $8,2 \%$ & 9 & $12,9 \%$ & 1,15 & ns \\
\hline ja, an anderen Orten & 273 & 24,3 & 258 & $24,5 \%$ & 15 & $21,4 \%$ & 0,61 & ns \\
\hline nein & 704 & 62,7 & 662 & $62,9 \%$ & 42 & $60,0 \%$ & 0,48 & ns \\
\hline an 2 Orten & 98 & 8,7 & 93 & $8,8 \%$ & 5 & $7,1 \%$ & 0,53 & ns \\
\hline an 3 Orten & 19 & 1,7 & 19 & $1,8 \%$ & 0 & $0,0 \%$ & 4,40 & ns \\
\hline \multicolumn{9}{|l|}{ Wein, Sekt, Obstwein } \\
\hline ja & 15 & 1,3 & 13 & $1,2 \%$ & 2 & $2,9 \%$ & 0,80 & ns \\
\hline weniger als 1 Glas & 87 & 7,8 & 77 & $7,3 \%$ & 10 & $14,3 \%$ & 1,64 & ns \\
\hline $\begin{array}{l}\text { habe fast nie Wein etc. } \\
\text { getrunken }\end{array}$ & 1020 & 90,9 & 962 & $91,4 \%$ & 58 & $82,9 \%$ & 1,87 & ns \\
\hline ja, 1 Glas pro Tag & 10 & 0,9 & 9 & $0,9 \%$ & 1 & $1,4 \%$ & 0,40 & ns \\
\hline ja, 2 Gläser pro Tag & 1 & 0,1 & 0 & $0,0 \%$ & 1 & $1,4 \%$ & 1,01 & ns \\
\hline $\begin{array}{l}\text { ja, mehr als } 2 \text { Gläser } \\
\text { pro Tag }\end{array}$ & 0 & 0,0 & 0 & $0,0 \%$ & 0 & $0,0 \%$ & & \\
\hline & & & & & & & & \\
\hline
\end{tabular}


Reiten in der Schwangerschaft - Primiparae

Anhang Tab. A1

Entbindungen ab $37+0$ vs. Entbindungen bis $36+6$

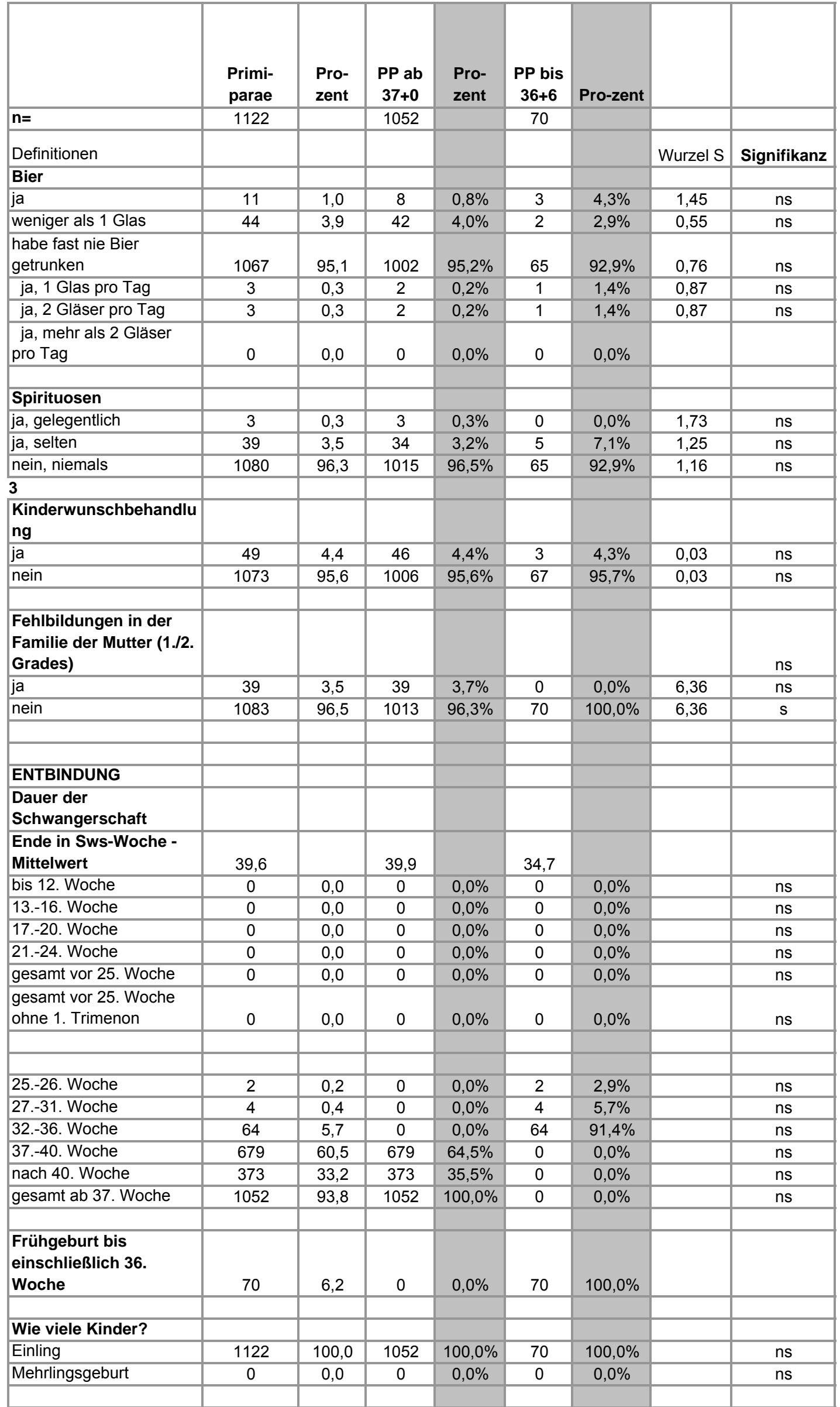


Reiten in der Schwangerschaft - Primiparae

Anhang Tab. A1

Entbindungen ab $37+0$ vs. Entbindungen bis $36+6$

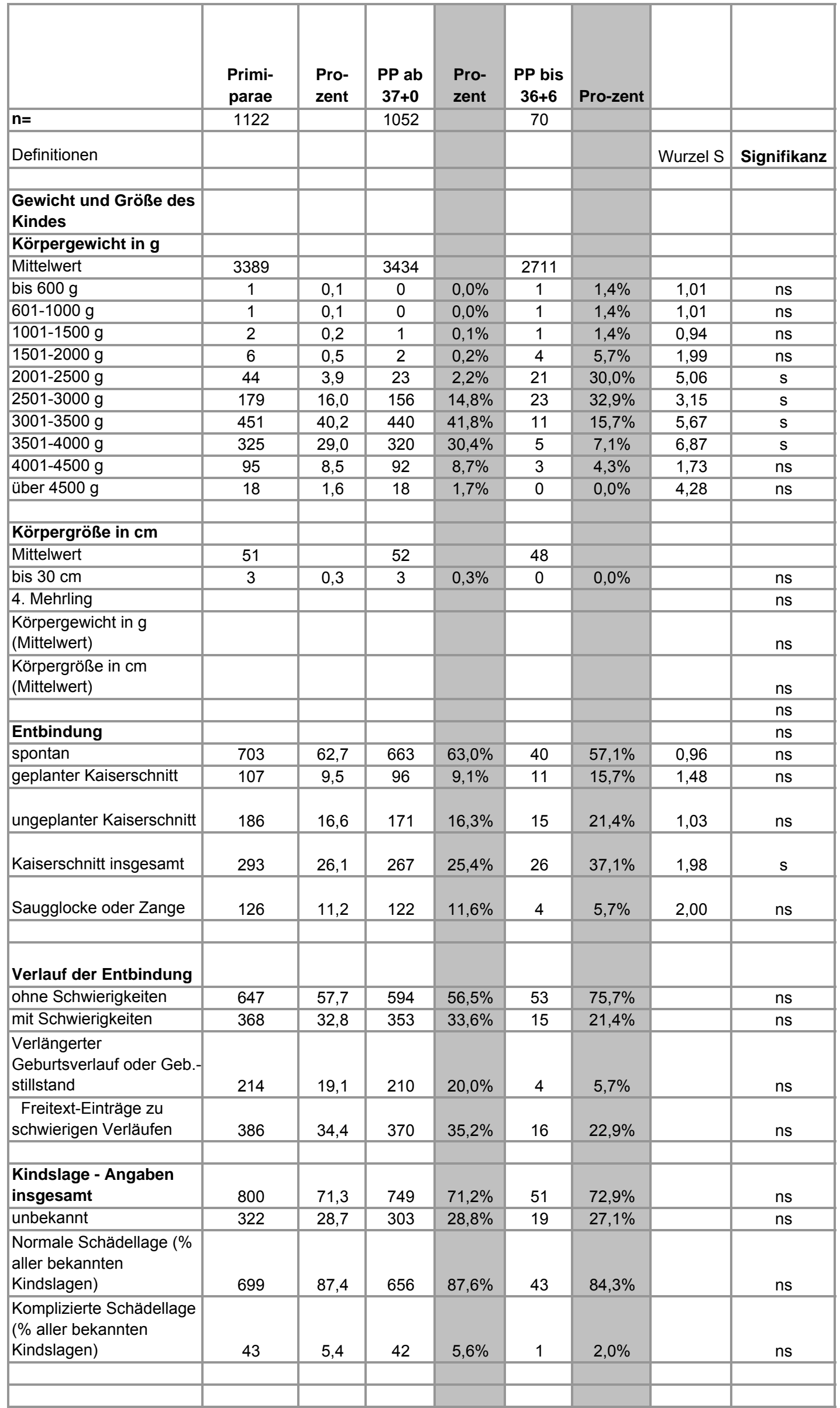


Reiten in der Schwangerschaft - Primiparae

Anhang Tab. A1

Entbindungen ab $37+0$ vs. Entbindungen bis $36+6$

\begin{tabular}{|c|c|c|c|c|c|c|c|c|}
\hline & $\begin{array}{l}\text { Primi- } \\
\text { parae }\end{array}$ & $\begin{array}{l}\text { Pro- } \\
\text { zent }\end{array}$ & $\begin{array}{c}\text { PP ab } \\
37+0\end{array}$ & $\begin{array}{l}\text { Pro- } \\
\text { zent }\end{array}$ & $\begin{array}{l}\text { PP bis } \\
36+6\end{array}$ & Pro-zent & & \\
\hline$n=$ & 1122 & & 1052 & & 70 & & & \\
\hline Definitionen & & & & & & & Wurzel S & Signifikanz \\
\hline $\begin{array}{l}\text { Beckenendlage (\% aller } \\
\text { bekannten Kindslagen) }\end{array}$ & 51 & 6,4 & 44 & $5,9 \%$ & 7 & $13,7 \%$ & & ns \\
\hline $\begin{array}{l}\text { Quer- oder Schräglage } \\
\text { (\% aller bekannten } \\
\text { Kindslagen) }\end{array}$ & 7 & 0,9 & 7 & $0,9 \%$ & 0 & $0,0 \%$ & & ns \\
\hline \multicolumn{9}{|l|}{ Kind nach der Geburt } \\
\hline gesund geboren & 1061 & 94,6 & 1000 & $95,1 \%$ & 61 & $87,1 \%$ & 1,95 & ns \\
\hline krank geboren & 61 & 5,4 & 52 & $4,9 \%$ & 9 & $12,9 \%$ & 1,95 & ns \\
\hline Totgeburt & 0 & 0,0 & 0 & $0,0 \%$ & 0 & $0,0 \%$ & & ns \\
\hline Freitext-Einträge & 60 & 5,3 & 51 & $4,8 \%$ & 9 & $12,9 \%$ & & ns \\
\hline $\begin{array}{l}\text { Kind (er) nach einem } \\
\text { Jahr }\end{array}$ & & & & & & & & ns \\
\hline Kind (alle Kinder) gesund & 1096 & 97,7 & 1028 & $97,7 \%$ & 68 & $97,1 \%$ & & ns \\
\hline $\begin{array}{l}\text { Kind (bzw. eines der } \\
\text { Kinder) krank }\end{array}$ & 23 & 2,0 & 22 & $2,1 \%$ & 1 & $1,4 \%$ & & ns \\
\hline $\begin{array}{l}\text { Kind (bzw. eines der } \\
\text { Kinder) verstorben }\end{array}$ & 2 & 0,2 & 1 & $0,1 \%$ & 1 & $1,4 \%$ & & ns \\
\hline Freitext-Einträge & 20 & 1,8 & 20 & $1,9 \%$ & 0 & $0,0 \%$ & & ns \\
\hline \multicolumn{9}{|l|}{$\begin{array}{l}\text { Jahrgang der } \\
\text { Entbindung }\end{array}$} \\
\hline 2005 & 169 & 15,1 & 163 & $15,5 \%$ & 6 & $8,6 \%$ & & ns \\
\hline 2004 & 244 & 21,7 & 225 & $21,4 \%$ & 19 & $27,1 \%$ & & $\mathrm{~ns}$ \\
\hline 2003 & 160 & 14,3 & 148 & $14,1 \%$ & 12 & $17,1 \%$ & & ns \\
\hline 2002 & 134 & 11,9 & 128 & $12,2 \%$ & 6 & $8,6 \%$ & & ns \\
\hline 2001 & 92 & 8,2 & 85 & $8,1 \%$ & 7 & $10,0 \%$ & & ns \\
\hline 2000 & 82 & 7,3 & 79 & $7,5 \%$ & 3 & $4,3 \%$ & & ns \\
\hline 1999 & 60 & 5,3 & 56 & $5,3 \%$ & 4 & $5,7 \%$ & & ns \\
\hline 1998 & 50 & 4,5 & 50 & $4,8 \%$ & 0 & $0,0 \%$ & & $\mathrm{~ns}$ \\
\hline 1997 & 64 & 5,7 & 60 & $5,7 \%$ & 4 & $5,7 \%$ & & ns \\
\hline 1996 & 33 & 2,9 & 28 & $2,7 \%$ & 5 & $7,1 \%$ & & ns \\
\hline 1995 & 34 & 3,0 & 30 & $2,9 \%$ & 4 & $5,7 \%$ & & ns \\
\hline
\end{tabular}


Reiten in der Schwangerschaft - Primiparae

Entbindungen bis $36+6$ vs. Entbindungen ab $37+0$, verdichtet

\begin{tabular}{|c|c|c|c|c|c|c|c|c|}
\hline & Absolut PP & Prozent & $\begin{array}{c}\text { Absolut PP } \\
\text { FG nein }\end{array}$ & \multirow[t]{2}{*}{ Prozent } & $\begin{array}{l}\text { Absolut } \\
\text { PP FG ja }\end{array}$ & \multirow[t]{2}{*}{ Prozent } & \multirow[b]{2}{*}{ T-WERT } & \multirow[b]{3}{*}{ Signifikanz } \\
\hline$n=$ & 1122 & & 1052 & & 70 & & & \\
\hline Angabe & & & & & & \multirow[b]{2}{*}{$52,9 \%$} & Wurzel S & \\
\hline Krankenhausaufenthalt wg. Komplikationen & 158 & 14,1 & 121 & $11,5 \%$ & 37 & & 6,84 & $\mathrm{~s}$ \\
\hline nein & 1083 & 96,5 & 1013 & $96,3 \%$ & 70 & $100,0 \%$ & 6,36 & $\mathrm{~s}$ \\
\hline später & 54 & 4,8 & 37 & $3,5 \%$ & 17 & $24,3 \%$ & 4,03 & $\mathrm{~s}$ \\
\hline Komplikationen, aber ohne Krankenhaus & 434 & 38,7 & 420 & $39,9 \%$ & 14 & $20,0 \%$ & 3,97 & s \\
\hline bis 7 Tage & 98 & 8,7 & 78 & $7,4 \%$ & 20 & $28,6 \%$ & 3,88 & s \\
\hline ja & 559 & 49,8 & 510 & $48,5 \%$ & 49 & $70,0 \%$ & 3,78 & $\mathrm{~s}$ \\
\hline nein & 1108 & 98,8 & 1038 & $98,7 \%$ & 70 & $100,0 \%$ & 3,77 & $\mathrm{~s}$ \\
\hline nein & 1111 & 99,0 & 1041 & $99,0 \%$ & 70 & $100,0 \%$ & 3,33 & s \\
\hline nein & 1098 & 97,9 & 1036 & $98,5 \%$ & 62 & $88,6 \%$ & 2,59 & $\mathrm{~s}$ \\
\hline keine Turnierteilnahmen mehr & 237 & 21,1 & 229 & $21,8 \%$ & 8 & $11,4 \%$ & 2,58 & s \\
\hline nein & 1065 & 94,9 & 996 & $94,7 \%$ & 69 & $98,6 \%$ & 2,47 & $\mathrm{~s}$ \\
\hline LK 4 & 89 & 7,9 & 76 & $7,2 \%$ & 13 & $18,6 \%$ & 2,41 & $\mathrm{~s}$ \\
\hline während der Schwangerschaft & 57 & 5,1 & 47 & $4,5 \%$ & 10 & $14,3 \%$ & 2,32 & $\mathrm{~s}$ \\
\hline mittlere Belastung & 180 & 16,0 & 174 & $16,5 \%$ & 6 & $8,6 \%$ & 2,25 & $\mathrm{~s}$ \\
\hline 8-14 Tage & 38 & 3,4 & 30 & $2,9 \%$ & 8 & $11,4 \%$ & 2,24 & $\mathrm{~s}$ \\
\hline selten & 360 & 32,1 & 345 & $32,8 \%$ & 15 & $21,4 \%$ & 2,22 & $\mathrm{~s}$ \\
\hline 21.-28. Woche & 18 & 1,6 & 12 & $1,1 \%$ & 6 & $8,6 \%$ & 2,21 & $\mathrm{~s}$ \\
\hline später & 39 & 3,5 & 31 & $2,9 \%$ & 8 & $11,4 \%$ & 2,21 & $\mathrm{~s}$ \\
\hline nein & 1071 & 95,5 & 1010 & $96,0 \%$ & 61 & $87,1 \%$ & 2,19 & s \\
\hline später & 88 & 7,8 & 76 & $7,2 \%$ & 12 & $17,1 \%$ & 2,17 & s \\
\hline
\end{tabular}




\begin{tabular}{|c|c|c|c|c|c|}
\hline & $\begin{array}{c}\text { Alle } \\
\text { Teilnehmeri } \\
\text { nnen, ET ab } \\
25 \text { SSW }\end{array}$ & & $\begin{array}{c}\text { Aborte und } \\
\text { Fehlgeburten bis } \\
24+6\end{array}$ & & \\
\hline$n=$ & 1800 & & 16 & & \\
\hline & & & & & Signifikanz \\
\hline \multicolumn{6}{|l|}{ Alter zu Beginn des SS } \\
\hline > 18 Jahre & 11 & $0,6 \%$ & 1 & $6,3 \%$ & ns \\
\hline 18 bis 24 Jahre & 243 & $13,5 \%$ & 2 & $12,5 \%$ & ns \\
\hline 25-29 Jahre & 652 & $36,2 \%$ & 5 & $31,3 \%$ & ns \\
\hline 30-34 Jahre & 658 & $36,6 \%$ & 5 & $31,3 \%$ & ns \\
\hline 35-39 Jahre & 215 & $11,9 \%$ & 3 & $18,8 \%$ & ns \\
\hline 40 Jahre und mehr & 21 & $1,2 \%$ & 0 & $0,0 \%$ & ns \\
\hline Gesamt & 1800 & $100,0 \%$ & 16 & $100,0 \%$ & \\
\hline Mittelwert & 29,5 & & 28,8 & & \\
\hline s & 4,6 & & 6,0 & & \\
\hline \multicolumn{6}{|l|}{ BMI } \\
\hline Untergewicht $<20$ & 365 & $20,3 \%$ & 6 & $37,5 \%$ & ns \\
\hline Normalgewicht $20-24,9$ & 1161 & $64,5 \%$ & 10 & $62,5 \%$ & ns \\
\hline Übergewicht > 25 & 272 & $15,1 \%$ & 0 & $0,0 \%$ & ns \\
\hline Summe & 0 & $0,0 \%$ & & & \\
\hline \multicolumn{6}{|l|}{ Frühere Kompl.: } \\
\hline vorzeit. Blasensprung & 66 & $3,7 \%$ & 0 & $0,0 \%$ & ns \\
\hline Frühgeburt/en & 75 & $4,2 \%$ & 0 & $0,0 \%$ & ns \\
\hline schwierige Schwangerschaft & 103 & $5,7 \%$ & 3 & $18,8 \%$ & ns \\
\hline schwierige Geburt & 154 & $8,6 \%$ & 0 & $0,0 \%$ & ns \\
\hline Kaiserschnitt & 91 & $5,1 \%$ & 0 & $0,0 \%$ & \\
\hline gyn. Operation & 102 & $5,7 \%$ & 1 & $6,3 \%$ & ns \\
\hline Krankenhausaufenthalt mind. 2 Wochen vor Sws & 15 & $0,8 \%$ & 0 & $0,0 \%$ & ns \\
\hline Summe der Nennungen & 606 & $33,7 \%$ & 4 & $25,0 \%$ & ns \\
\hline 1 Kompl. Angegeben & 279 & $15,5 \%$ & 4 & $25,0 \%$ & ns \\
\hline 2 Komplik. angegeben & 60 & $3,3 \%$ & 0 & $0,0 \%$ & ns \\
\hline 3 Komplat. Angegeben & 16 & $0,9 \%$ & 0 & $0,0 \%$ & ns \\
\hline 4 und mehr Komplik. Angegeben & 3 & $0,2 \%$ & 0 & $0,0 \%$ & ns \\
\hline Gesamt mit Komplikationen & 358 & $19,9 \%$ & 4 & $25,0 \%$ & ns \\
\hline \multicolumn{6}{|l|}{ Schulabschluss } \\
\hline Schule noch nicht beendet & 11 & $0,6 \%$ & 0 & $0,0 \%$ & ns \\
\hline Schule beendet ohne Abschluss & 1 & $0,1 \%$ & 0 & $0,0 \%$ & ns \\
\hline Haupt/ Volksschulabschluss & 95 & $5,3 \%$ & 1 & $6,3 \%$ & ns \\
\hline Realschulabschluss & 515 & $28,6 \%$ & 2 & $12,5 \%$ & ns \\
\hline Abschluss Polytechnische Oberschule & 45 & $2,5 \%$ & 0 & $0,0 \%$ & ns \\
\hline Fachhochschulreife & 204 & $11,3 \%$ & 1 & $6,3 \%$ & ns \\
\hline Allg. oder fachgebundene Hochschulreife & 929 & $51,6 \%$ & 12 & $75,0 \%$ & $\mathrm{~s}$ \\
\hline
\end{tabular}




\begin{tabular}{|c|c|c|c|c|c|}
\hline & $\begin{array}{c}\text { Alle } \\
\text { Teilnehmeri } \\
\text { nnen, ET ab } \\
25 \text { SSW }\end{array}$ & & $\begin{array}{c}\text { Aborte und } \\
\text { Fehlgeburten bis } \\
24+6\end{array}$ & & \\
\hline$n=$ & 1800 & & 16 & & \\
\hline & & & & & Signifikanz \\
\hline \multicolumn{6}{|l|}{$\begin{array}{l}\text { Sportlicher Trainingszustand vor der } \\
\text { Schwangerschaft }\end{array}$} \\
\hline ausgezeichnet (täglich mind. $1 \mathrm{~h}$ Sport) & 428 & $23,8 \%$ & 2 & $12,5 \%$ & ns \\
\hline sehr gut (4h und mehr Sport pro Woche) & 520 & $28,9 \%$ & 3 & $18,8 \%$ & ns \\
\hline gut (2-4h Sport pro Woche) & 569 & $31,6 \%$ & 9 & $56,3 \%$ & $\mathrm{~s}$ \\
\hline mäßig (1-2 h Sport pro Woche) & 204 & $11,3 \%$ & 0 & $0,0 \%$ & ns \\
\hline nicht besonders (weniger als $1 \mathrm{~h}$ Sport pro Wo.) & 79 & $4,4 \%$ & 2 & $12,5 \%$ & \\
\hline Sportarten vor der Schwangerschaft & & & & & ns \\
\hline keine Sportarten zusätzlich zum Reiten & 705 & $39,2 \%$ & 4 & $25,0 \%$ & ns \\
\hline Insgesamt sportl. aktiv zusätzlich zum Reiten & 1095 & $60,8 \%$ & 12 & $75,0 \%$ & ns \\
\hline Ausdauerbetonte Sportarten & 773 & $42,9 \%$ & 11 & $68,8 \%$ & $\mathrm{~s}$ \\
\hline Kraftbetonte Sportarten & 126 & $7,0 \%$ & 0 & $0,0 \%$ & ns \\
\hline Gymnastische Sportarten & 203 & $11,3 \%$ & 2 & $12,5 \%$ & ns \\
\hline Fun- und Risikosportarten & 259 & $14,4 \%$ & 7 & $43,8 \%$ & $\mathrm{~s}$ \\
\hline Spielsportarten & 103 & $5,7 \%$ & 0 & $0,0 \%$ & ns \\
\hline nur 1 Sportart & 767 & $42,6 \%$ & & & \\
\hline 2 Sportarten & 283 & $15,7 \%$ & & & \\
\hline 3 oder 4 Sportarten & 45 & $2,5 \%$ & & & \\
\hline \multicolumn{6}{|l|}{ Leistungsstand Reiten } \\
\hline Ungeübte Reiterin & 42 & $2,3 \%$ & 0 & $0,0 \%$ & ns \\
\hline Geübte Reiterin & 1188 & $66,0 \%$ & 14 & $87,5 \%$ & ns \\
\hline Leistungssportlerin im gehobenen Bereich & 494 & $27,4 \%$ & 2 & $12,5 \%$ & ns \\
\hline Berufsreiterin & 76 & $4,2 \%$ & 0 & $0,0 \%$ & ns \\
\hline \multicolumn{6}{|l|}{ Leistungsklasse } \\
\hline ohne & 1250 & $69,4 \%$ & 16 & $100,0 \%$ & $\mathrm{~s}$ \\
\hline LK 1 & 5 & $0,3 \%$ & 0 & $0,0 \%$ & ns \\
\hline LK 2 & 16 & $0,9 \%$ & 0 & $0,0 \%$ & ns \\
\hline LK 3 & 64 & $3,6 \%$ & 0 & $0,0 \%$ & ns \\
\hline LK 4 & 159 & $8,8 \%$ & 1 & $6,3 \%$ & ns \\
\hline LK 5 & 194 & $10,8 \%$ & 0 & $0,0 \%$ & ns \\
\hline LK 6 & 42 & $2,3 \%$ & 1 & $6,3 \%$ & ns \\
\hline \multicolumn{6}{|l|}{ Reittage pro Woche vor der SWS } \\
\hline nur gelegentlich oder im Urlaub & 26 & $1,4 \%$ & 0 & $0,0 \%$ & ns \\
\hline 1x pro Woche & 55 & $3,1 \%$ & 2 & $12,5 \%$ & ns \\
\hline 2x pro Woche & 226 & $12,6 \%$ & 0 & $0,0 \%$ & ns \\
\hline 3-5x pro Woche & 837 & $46,5 \%$ & 10 & $62,5 \%$ & ns \\
\hline täglich & 656 & $36,4 \%$ & 4 & $25,0 \%$ & ns \\
\hline
\end{tabular}




\section{Reiten in der Schwangerschaft}

Aborte

Schwangerschaften mit Beendigung bis $24+6$ vs. Beendigung ab $25+0$

\begin{tabular}{|c|c|c|c|c|c|}
\hline & $\begin{array}{c}\text { Alle } \\
\text { Teilnehmeri } \\
\text { nnen, ET ab } \\
25 \text { SSW }\end{array}$ & & $\begin{array}{l}\text { Aborte und } \\
\text { Fehlgeburten bis } \\
24+6\end{array}$ & & \\
\hline$n=$ & 1800 & & 16 & & \\
\hline & & & & & Signifikanz \\
\hline Tägliche Reitdauer vor der SWS & & & & & ns \\
\hline $1 \mathrm{~h}$ & 1191 & $66,2 \%$ & 13 & $81,3 \%$ & ns \\
\hline $2-3 \mathrm{~h}$ & 542 & $30,1 \%$ & 3 & $18,8 \%$ & ns \\
\hline $4 \mathrm{~h}$ und mehr & 67 & $3,7 \%$ & 0 & $0,0 \%$ & ns \\
\hline \multicolumn{6}{|l|}{ Dressur } \\
\hline nicht geritten & 263 & $14,6 \%$ & 1 & $6,3 \%$ & ns \\
\hline leichte Belastung & 233 & $12,9 \%$ & 3 & $18,8 \%$ & ns \\
\hline mittlere Belastung & 490 & $27,2 \%$ & 9 & $56,3 \%$ & $\mathrm{~s}$ \\
\hline leistungsorientiertes Reiten & 693 & $38,5 \%$ & 3 & $18,8 \%$ & ns \\
\hline Spitzensport & 120 & $6,7 \%$ & 0 & $0,0 \%$ & ns \\
\hline \multicolumn{6}{|l|}{ Springen } \\
\hline nicht geritten & 878 & $48,8 \%$ & 9 & $56,3 \%$ & ns \\
\hline leichte Belastung & 345 & $19,2 \%$ & 3 & $18,8 \%$ & ns \\
\hline mittlere Belastung & 277 & $15,4 \%$ & 1 & $6,3 \%$ & ns \\
\hline leistungsorientiertes Reiten & 261 & $14,5 \%$ & 3 & $18,8 \%$ & ns \\
\hline Spitzensport & 39 & $2,2 \%$ & 0 & $0,0 \%$ & ns \\
\hline \multicolumn{6}{|l|}{ Ausreiten } \\
\hline nicht geritten & 82 & $4,6 \%$ & 1 & $6,3 \%$ & ns \\
\hline leichte Belastung & 362 & $20,1 \%$ & 6 & $37,5 \%$ & ns \\
\hline mittlere Belastung & 914 & $50,8 \%$ & 5 & $31,3 \%$ & ns \\
\hline leistungsorientiertes Reiten & 389 & $21,6 \%$ & 3 & $18,8 \%$ & ns \\
\hline Spitzensport & 53 & $2,9 \%$ & 1 & $6,3 \%$ & ns \\
\hline \multicolumn{6}{|l|}{ Gelände I Vielseitigkeit } \\
\hline nicht geritten & 1146 & $63,7 \%$ & 12 & $75,0 \%$ & ns \\
\hline leichte Belastung & 217 & $12,1 \%$ & 2 & $12,5 \%$ & ns \\
\hline mittlere Belastung & 280 & $15,6 \%$ & 2 & $12,5 \%$ & ns \\
\hline leistungsorientiertes Reiten & 138 & $7,7 \%$ & 0 & $0,0 \%$ & ns \\
\hline Spitzensport & 19 & $1,1 \%$ & 0 & $0,0 \%$ & ns \\
\hline \multicolumn{6}{|l|}{ Gangpferde } \\
\hline nicht geritten & 1658 & $92,1 \%$ & 14 & $87,5 \%$ & ns \\
\hline leichte Belastung & 35 & $1,9 \%$ & 0 & $0,0 \%$ & ns \\
\hline mittlere Belastung & 50 & $2,8 \%$ & 2 & $12,5 \%$ & ns \\
\hline leistungsorientiertes Reiten & 42 & $2,3 \%$ & 0 & $0,0 \%$ & ns \\
\hline Spitzensport & 15 & $0,8 \%$ & 0 & $0,0 \%$ & ns \\
\hline \multicolumn{6}{|l|}{ Western } \\
\hline nicht geritten & 1552 & $86,2 \%$ & 13 & $81,3 \%$ & ns \\
\hline leichte Belastung & 67 & $3,7 \%$ & 1 & $6,3 \%$ & ns \\
\hline mittlere Belastung & 104 & $5,8 \%$ & 1 & $6,3 \%$ & ns \\
\hline leistungsorientiertes Reiten & 71 & $3,9 \%$ & 1 & $6,3 \%$ & ns \\
\hline Spitzensport & 6 & $0,3 \%$ & 0 & $0,0 \%$ & ns \\
\hline
\end{tabular}




\section{Reiten in der Schwangerschaft}

Aborte

Schwangerschaften mit Beendigung bis $24+6$ vs. Beendigung ab $25+0$

\begin{tabular}{|c|c|c|c|c|c|}
\hline & $\begin{array}{c}\text { Alle } \\
\text { Teilnehmeri } \\
\text { nnen, ET ab } \\
\quad 25 \text { SSW }\end{array}$ & & $\begin{array}{l}\text { Aborte und } \\
\text { Fehlgeburten bis } \\
24+6\end{array}$ & & \\
\hline$n=$ & 1800 & & 16 & & \\
\hline & & & & & Signifikanz \\
\hline \multicolumn{6}{|l|}{ Distanzreiten } \\
\hline nicht geritten & 1649 & $91,6 \%$ & 14 & $87,5 \%$ & ns \\
\hline leichte Belastung & 60 & $3,3 \%$ & 1 & $6,3 \%$ & ns \\
\hline mittlere Belastung & 51 & $2,8 \%$ & 1 & $6,3 \%$ & ns \\
\hline leistungsorientiertes Reiten & 29 & $1,6 \%$ & 0 & $0,0 \%$ & ns \\
\hline Spitzensport & 11 & $0,6 \%$ & 0 & $0,0 \%$ & ns \\
\hline \multicolumn{6}{|l|}{ Rennsport } \\
\hline nicht geritten & 1772 & $98,4 \%$ & 16 & $100,0 \%$ & ns \\
\hline leichte Belastung & 13 & $0,7 \%$ & 0 & $0,0 \%$ & ns \\
\hline mittlere Belastung & 8 & $0,4 \%$ & 0 & $0,0 \%$ & ns \\
\hline leistungsorientiertes Reiten & 6 & $0,3 \%$ & 0 & $0,0 \%$ & ns \\
\hline Spitzensport & 1 & $0,1 \%$ & 0 & $0,0 \%$ & ns \\
\hline Information des Frauenarztes & & & & & ns \\
\hline ja, bereits vor der Schwangerschaft & 733 & $40,7 \%$ & 5 & $31,3 \%$ & ns \\
\hline ja, zu Beginn der Schwangerschaft & 819 & $45,5 \%$ & 8 & $50,0 \%$ & ns \\
\hline ja, im späteren Verlauf der Schwangerschaft & 86 & $4,8 \%$ & 0 & $0,0 \%$ & ns \\
\hline nein & 162 & $9,0 \%$ & 3 & $18,8 \%$ & ns \\
\hline \multicolumn{6}{|l|}{ Empfehlung des Frauenarztes } \\
\hline hat abgeraten & 221 & $12,3 \%$ & 6 & $37,5 \%$ & ns \\
\hline Entscheidung der Schwangeren überlassen & 1125 & $62,5 \%$ & 6 & $37,5 \%$ & ns \\
\hline Weiterreiten empfohlen & 292 & $16,2 \%$ & 1 & $6,3 \%$ & ns \\
\hline Frauenarzt war nicht informiert & 162 & $9,0 \%$ & 3 & $18,8 \%$ & ns \\
\hline \multicolumn{6}{|l|}{ In der Sws weitergeritten } \\
\hline $\mathrm{Ja}$ & 1668 & $92,7 \%$ & 12 & $75,0 \%$ & ns \\
\hline und zwar bis zur Schwangerschaftswoche & 28,73201439 & & 14,16666667 & & \\
\hline \multicolumn{6}{|l|}{ St-Abweichung } \\
\hline Nein & 128 & $7,1 \%$ & 4 & $25,0 \%$ & ns \\
\hline aufgehört in Schwangerschaftswoche & 73 & & 0 & & \\
\hline \multicolumn{6}{|l|}{ St-Abweichung } \\
\hline \multicolumn{6}{|l|}{ Beschwerden im Zusammenhang mit dem Reiten } \\
\hline 1. Trimenon harter Bauch od. Ziehen im Unterleib & 72 & $3,9 \%$ & 72 & $3,9 \%$ & ns \\
\hline 2. Trimenon Beschwerden & 122 & $6,8 \%$ & 0 & $0,0 \%$ & ns \\
\hline 3. Trimenon Beschwerden & 201 & $11,2 \%$ & 0 & $0,0 \%$ & ns \\
\hline 1.und 2. Trimenon Beschwerden & 29 & $1,6 \%$ & 0 & $0,0 \%$ & ns \\
\hline 1. und 3. Trimenon Beschwerden & 2 & $0,1 \%$ & 0 & $0,0 \%$ & ns \\
\hline 2. und 3. Trimenon Beschwerden & 53 & $2,9 \%$ & 0 & $0,0 \%$ & ns \\
\hline 1.,2.,3. Trimenon Beschwerden & 20 & $1,1 \%$ & 0 & $0,0 \%$ & ns \\
\hline
\end{tabular}




\section{Reiten in der Schwangerschaft}

Aborte

Schwangerschaften mit Beendigung bis $24+6$ vs. Beendigung ab $25+0$

\begin{tabular}{|c|c|c|c|c|c|}
\hline & $\begin{array}{c}\text { Alle } \\
\text { Teilnehmeri } \\
\text { nnen, ET ab } \\
25 \text { SSW }\end{array}$ & & $\begin{array}{l}\text { Aborte und } \\
\text { Fehlgeburten bis } \\
24+6\end{array}$ & & \\
\hline$n=$ & 1800 & & 16 & & \\
\hline & & & & & Signifikanz \\
\hline Gesamt keine Beschwerden & 1306 & $72,6 \%$ & 11 & $68,8 \%$ & ns \\
\hline Gesamt Beschwerden & 494,00 & $27,4 \%$ & 5,00 & $31,3 \%$ & ns \\
\hline \multicolumn{6}{|l|}{ Ich habe mit dem Reiten aufgehört, } \\
\hline erst als das Reiten unbequem wurde & 1010 & $56,1 \%$ & 0 & $0,0 \%$ & ns \\
\hline weil ich Angst hatte, mir oder dem Kind zu schaden & 381 & $21,2 \%$ & 4 & $25,0 \%$ & ns \\
\hline $\begin{array}{l}\text { weil ich vorzeitige Wehen, Blutungen oder andere } \\
\text { Probleme mit der Schwangerschaft bekommen habe }\end{array}$ & 155 & $8,6 \%$ & 1 & $6,3 \%$ & ns \\
\hline $\begin{array}{l}\text { weil der Frauenarzt oder eine andere Person mir das } \\
\text { Aufhören dringend nahe gelegt hat }\end{array}$ & 125 & $6,9 \%$ & 2 & $12,5 \%$ & ns \\
\hline weil ich krank geworden bin & 37 & $2,1 \%$ & 3 & $18,8 \%$ & ns \\
\hline andere Gründe & 308 & $17,1 \%$ & 5 & $31,3 \%$ & ns \\
\hline bis zur Geburt weitergeritten & 214 & $11,9 \%$ & 8 & $50,0 \%$ & $\mathrm{~s}$ \\
\hline erst als das Reiten unbequem wurde - allein & 740 & $41,1 \%$ & 0 & $0,0 \%$ & ns \\
\hline $\begin{array}{l}\text { weil ich vorzeitige Wehen, etc. bekommen habe - } \\
\text { allein }\end{array}$ & 84 & $4,7 \%$ & 0 & $0,0 \%$ & ns \\
\hline $\begin{array}{l}\text { weil ich Angst hatte, mir oder dem Kind zu schaden - } \\
\text { allein }\end{array}$ & 163 & $9,1 \%$ & 1 & $6,3 \%$ & ns \\
\hline $\begin{array}{l}\text { weil jemand mir das Aufhören dringend nahe gelegt } \\
\text { hat - allein }\end{array}$ & 40 & $2,2 \%$ & 0 & $0,0 \%$ & ns \\
\hline Angst hatte UND Aufhören nahe gelegt & 27 & $1,5 \%$ & 2 & $12,5 \%$ & ns \\
\hline weil ich krank geworden bin - allein & 14 & $0,8 \%$ & 2 & $12,5 \%$ & ns \\
\hline zwei Gründe angegeben & 388 & $21,6 \%$ & 5 & $31,3 \%$ & ns \\
\hline drei Gründe angegeben & 40 & $2,2 \%$ & 0 & $0,0 \%$ & ns \\
\hline \multicolumn{6}{|l|}{ Trotz Warnzeichen weitergeritten ... } \\
\hline ja & 123 & $6,8 \%$ & 3 & $18,8 \%$ & ns \\
\hline nein & 1453 & $80,7 \%$ & 9 & $56,3 \%$ & $\mathrm{~s}$ \\
\hline keine Angabe & 224 & $12,4 \%$ & 4 & $25,0 \%$ & ns \\
\hline \multicolumn{6}{|l|}{ Reittage pro Woche während der SWS } \\
\hline nur gelegentlich oder im Urlaub & 51 & $2,8 \%$ & 1 & $6,3 \%$ & ns \\
\hline 1x pro Woche & 131 & $7,3 \%$ & 2 & $12,5 \%$ & ns \\
\hline 2x pro Woche & 459 & $25,5 \%$ & 5 & $31,3 \%$ & ns \\
\hline $3-5 x$ pro Woche & 845 & $46,9 \%$ & 5 & $31,3 \%$ & ns \\
\hline täglich & 305 & $16,9 \%$ & 3 & $18,8 \%$ & ns \\
\hline nicht weitergeritten & 0 & $0,0 \%$ & 0 & $0,0 \%$ & \\
\hline Insges. Während SWS weitergeritten & 1791 & $99,5 \%$ & 16 & $100,0 \%$ & $\mathrm{~s}$ \\
\hline
\end{tabular}


Schwangerschaften mit Beendigung bis $24+6$ vs. Beendigung ab $25+0$

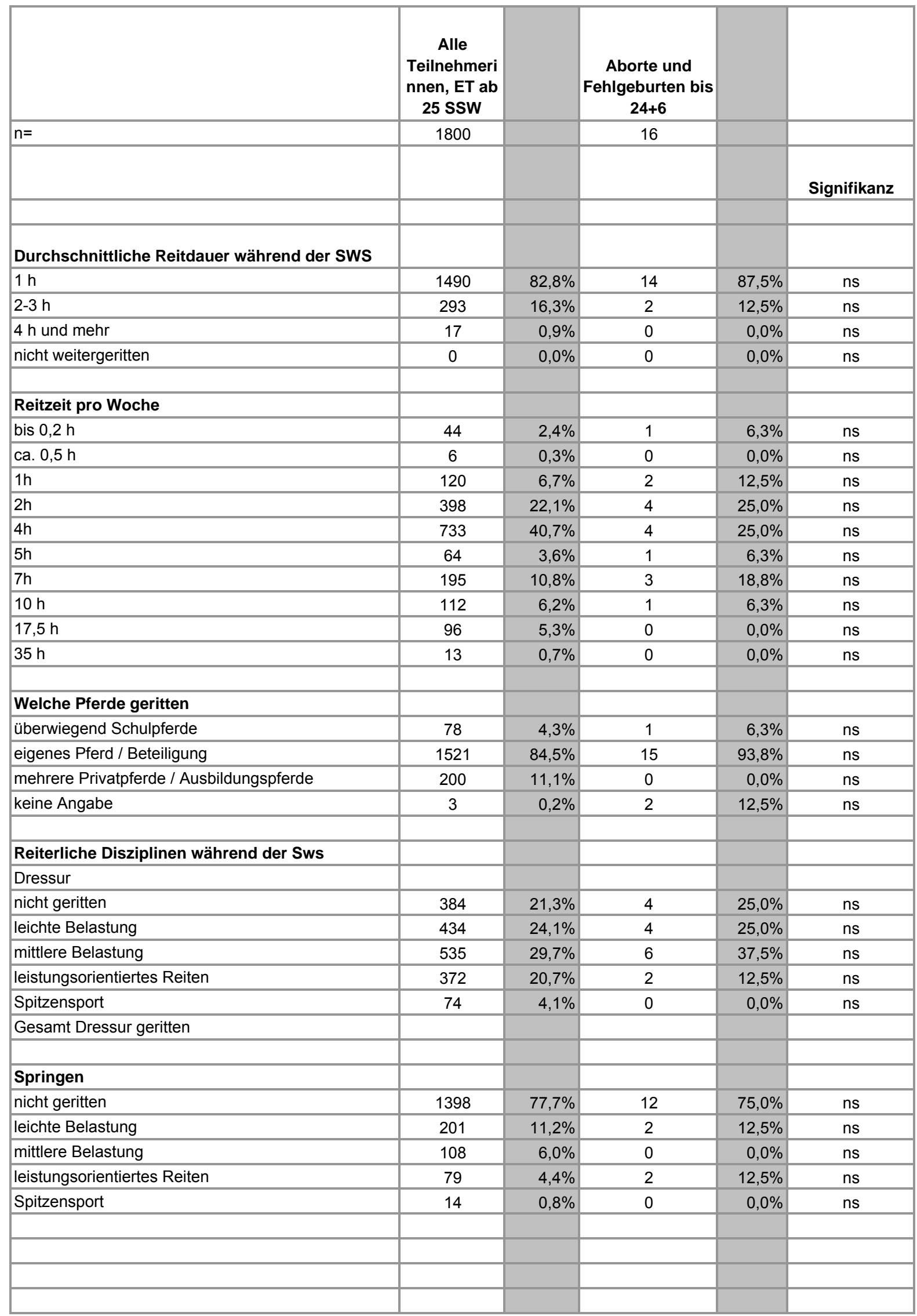


Schwangerschaften mit Beendigung bis $24+6$ vs. Beendigung ab $25+0$

\begin{tabular}{|c|c|c|c|c|c|}
\hline & $\begin{array}{c}\text { Alle } \\
\text { Teilnehmeri } \\
\text { nnen, ET ab } \\
25 \text { SSW }\end{array}$ & & $\begin{array}{c}\text { Aborte und } \\
\text { Fehlgeburten bis } \\
24+6\end{array}$ & & \\
\hline$n=$ & 1800 & & 16 & & \\
\hline & & & & & Signifikanz \\
\hline \multicolumn{6}{|l|}{ Ausreiten } \\
\hline nicht geritten & 210 & $11,7 \%$ & 4 & $25,0 \%$ & ns \\
\hline leichte Belastung & 587 & $32,6 \%$ & 6 & $37,5 \%$ & ns \\
\hline mittlere Belastung & 793 & $44,1 \%$ & 5 & $31,3 \%$ & ns \\
\hline leistungsorientiertes Reiten & 178 & $9,9 \%$ & 1 & $6,3 \%$ & ns \\
\hline Spitzensport & 32 & $1,8 \%$ & 0 & $0,0 \%$ & ns \\
\hline \multicolumn{6}{|l|}{ Gelände / Vielseitigkeit } \\
\hline nicht geritten & 1425 & $79,2 \%$ & 12 & $75,0 \%$ & ns \\
\hline leichte Belastung & 187 & $10,4 \%$ & 2 & $12,5 \%$ & ns \\
\hline mittlere Belastung & 144 & $8,0 \%$ & 2 & $12,5 \%$ & ns \\
\hline leistungsorientiertes Reiten & 36 & $2,0 \%$ & 0 & $0,0 \%$ & ns \\
\hline Spitzensport & 8 & $0,4 \%$ & 0 & $0,0 \%$ & ns \\
\hline \multicolumn{6}{|l|}{ Gangpferde } \\
\hline nicht geritten & 1690 & $93,9 \%$ & 14 & $87,5 \%$ & ns \\
\hline leichte Belastung & 34 & $1,9 \%$ & 0 & $0,0 \%$ & ns \\
\hline mittlere Belastung & 41 & $2,3 \%$ & 2 & $12,5 \%$ & ns \\
\hline leistungsorientiertes Reiten & 26 & $1,4 \%$ & 0 & $0,0 \%$ & ns \\
\hline Spitzensport & 9 & $0,5 \%$ & 0 & $0,0 \%$ & ns \\
\hline \multicolumn{6}{|l|}{ Western } \\
\hline nicht geritten & 1588 & $88,2 \%$ & 14 & $87,5 \%$ & ns \\
\hline leichte Belastung & 91 & $5,1 \%$ & 0 & $0,0 \%$ & ns \\
\hline mittlere Belastung & 86 & $4,8 \%$ & 1 & $6,3 \%$ & ns \\
\hline leistungsorientiertes Reiten & 33 & $1,8 \%$ & 1 & $6,3 \%$ & ns \\
\hline Spitzensport & 2 & $0,1 \%$ & 0 & $0,0 \%$ & ns \\
\hline \multicolumn{6}{|l|}{ Distanzreiten } \\
\hline nicht geritten & 1744 & $96,9 \%$ & 14 & $87,5 \%$ & ns \\
\hline leichte Belastung & 29 & $1,6 \%$ & 2 & $12,5 \%$ & ns \\
\hline mittlere Belastung & 18 & $1,0 \%$ & 0 & $0,0 \%$ & ns \\
\hline leistungsorientiertes Reiten & 5 & $0,3 \%$ & 0 & $0,0 \%$ & ns \\
\hline Spitzensport & 4 & $0,2 \%$ & 0 & $0,0 \%$ & ns \\
\hline \multicolumn{6}{|l|}{ Rennsport } \\
\hline nicht geritten & 1786 & $99,2 \%$ & 16 & $100,0 \%$ & ns \\
\hline leichte Belastung & 0 & $0,0 \%$ & 0 & $0,0 \%$ & ns \\
\hline mittlere Belastung & 0 & $0,0 \%$ & 0 & $0,0 \%$ & \\
\hline leistungsorientiertes Reiten & 0 & $0,0 \%$ & 0 & $0,0 \%$ & \\
\hline Spitzensport & 0 & $0,0 \%$ & 0 & $0,0 \%$ & \\
\hline & & & & & \\
\hline & & & & & \\
\hline
\end{tabular}


Schwangerschaften mit Beendigung bis $24+6$ vs. Beendigung ab $25+0$

\begin{tabular}{|c|c|c|c|c|c|}
\hline & $\begin{array}{c}\text { Alle } \\
\text { Teilnehmeri } \\
\text { nnen, ET ab } \\
25 \text { SSW }\end{array}$ & & $\begin{array}{c}\text { Aborte und } \\
\text { Fehlgeburten bis } \\
24+6\end{array}$ & & \\
\hline$n=$ & 1800 & & 16 & & \\
\hline & & & & & Signifikanz \\
\hline \multicolumn{6}{|l|}{ Turnierteilnahmen während der Schwangerschaft } \\
\hline ja & 387 & $21,5 \%$ & 1 & $6,3 \%$ & ns \\
\hline nein & 1411 & $78,4 \%$ & 15 & $93,8 \%$ & $\mathrm{~s}$ \\
\hline keine Angabe & 4 & $0,2 \%$ & 2 & $12,5 \%$ & ns \\
\hline \multicolumn{6}{|l|}{ Junge Pferde I Problempferde geritten } \\
\hline ja & 441 & $24,5 \%$ & 4 & $25,0 \%$ & ns \\
\hline nein & 1353 & $75,2 \%$ & 12 & $75,0 \%$ & ns \\
\hline keine Angabe & 8 & $0,4 \%$ & 2 & $12,5 \%$ & ns \\
\hline \multicolumn{6}{|l|}{ Reitstil während der Schwangerschaft geändert } \\
\hline ja & 1156 & $64,2 \%$ & 5 & $31,3 \%$ & s \\
\hline nein & 640 & $35,6 \%$ & 11 & $68,8 \%$ & $\mathrm{~s}$ \\
\hline keine Angabe & 6 & $0,3 \%$ & 2 & $12,5 \%$ & ns \\
\hline \multicolumn{6}{|l|}{ Reitstil geändert, und zwar } \\
\hline weniger anstrengender Reitstil & 672 & $37,3 \%$ & 4 & $25,0 \%$ & ns \\
\hline im Trab kein Aussitzen, nur Leichttrab & 778 & $43,2 \%$ & 3 & $18,8 \%$ & ns \\
\hline im Galopp nur leichter Sitz & 413 & $22,9 \%$ & 2 & $12,5 \%$ & ns \\
\hline $\begin{array}{l}\text { weniger hohe Sprünge, weniger Tempo beim } \\
\text { Springen }\end{array}$ & 180 & $10,0 \%$ & 2 & $12,5 \%$ & ns \\
\hline keine Sprünge mehr & 524 & $29,1 \%$ & 1 & $6,3 \%$ & ns \\
\hline weniger Turnierteilnahmen & 139 & $7,7 \%$ & 1 & $6,3 \%$ & ns \\
\hline keine Turnierteilnahmen mehr & 365 & $20,3 \%$ & 0 & $0,0 \%$ & ns \\
\hline vermehrt ruhige Ausritte & 734 & $40,8 \%$ & 1 & $6,3 \%$ & ns \\
\hline sonstiges & 210 & $11,7 \%$ & 0 & $0,0 \%$ & ns \\
\hline keine Angabe & 634 & $35,2 \%$ & 12 & $75,0 \%$ & ns \\
\hline Insgesamt Reitstil geändert & 1168 & $64,9 \%$ & 6 & $37,5 \%$ & s \\
\hline \multicolumn{6}{|l|}{ Unfallrisiko } \\
\hline fühlte mich völlig sicher, Unfall ausgeschlossen & 884 & $49,1 \%$ & 8 & $50,0 \%$ & ns \\
\hline fühlte mich einigermaßen sicher, Unfallrisiko gering & 774 & $43,0 \%$ & 7 & $43,8 \%$ & ns \\
\hline teilweise Reiten mit gewissem Risiko & 124 & $6,9 \%$ & 1 & $6,3 \%$ & ns \\
\hline keine Angabe & 20 & $1,1 \%$ & 2 & $12,5 \%$ & ns \\
\hline \multicolumn{6}{|l|}{$\begin{array}{l}\text { Unfall beim Reiten oder beim Umgang mit dem } \\
\text { Pferd }\end{array}$} \\
\hline ja (10 Frauen mit Mehrfachnennungen) & 187 & $10,4 \%$ & 2 & $12,5 \%$ & ns \\
\hline nein & 1609 & $89,4 \%$ & 14 & $87,5 \%$ & ns \\
\hline $\begin{array}{l}\text { Kein Umgang mit Pferden während der } \\
\text { Schwangerschaft }\end{array}$ & 6 & $0,3 \%$ & 2 & $12,5 \%$ & ns \\
\hline
\end{tabular}


Schwangerschaften mit Beendigung bis $24+6$ vs. Beendigung ab $25+0$

\begin{tabular}{|c|c|c|c|c|c|}
\hline & $\begin{array}{c}\text { Alle } \\
\text { Teilnehmeri } \\
\text { nnen, ET ab } \\
25 \text { SSW }\end{array}$ & & $\begin{array}{c}\text { Aborte und } \\
\text { Fehlgeburten bis } \\
24+6\end{array}$ & & \\
\hline$n=$ & 1800 & & 16 & & \\
\hline & & & & & Signifikanz \\
\hline \multicolumn{6}{|l|}{ Unfälle mit dem Pferd } \\
\hline \multicolumn{6}{|l|}{ Stürze vom Pferd } \\
\hline 1 Sturz & 87 & $4,8 \%$ & 2 & $12,5 \%$ & ns \\
\hline 2 Stürze & 12 & $0,7 \%$ & 0 & $0,0 \%$ & ns \\
\hline 3 und mehr Stürze & 3 & $0,2 \%$ & 0 & $0,0 \%$ & ns \\
\hline keine Angabe & 1700 & $94,4 \%$ & 16 & $100,0 \%$ & ns \\
\hline \multicolumn{6}{|l|}{ Stürze mit dem Pferd } \\
\hline 1 Sturz & 37 & $2,1 \%$ & 0 & $0,0 \%$ & ns \\
\hline 2 Stürze & 4 & $0,2 \%$ & 0 & $0,0 \%$ & ns \\
\hline 3 und mehr Stürze & 0 & $0,0 \%$ & 0 & $0,0 \%$ & ns \\
\hline \multicolumn{6}{|l|}{ Unfälle im Umgang mit dem Pferd } \\
\hline 1 Unfall & 52 & $2,9 \%$ & 0 & $0,0 \%$ & ns \\
\hline 2 Unfälle & 2 & $0,1 \%$ & 0 & $0,0 \%$ & ns \\
\hline 3 und mehr Unfälle & 0 & $0,0 \%$ & 0 & $0,0 \%$ & ns \\
\hline kein Unfall & 2 & $0,1 \%$ & 0 & $0,0 \%$ & ns \\
\hline \multicolumn{6}{|l|}{ Unfallfolgen (\% bezogen auf Unfälle) } \\
\hline Unfall blieb ohne Folgen für die Schwangerschaft & 179 & $95,7 \%$ & 1 & $50,0 \%$ & ns \\
\hline Wehen nach dem Unfall ohne Geburtseintritt & 7 & $3,7 \%$ & 0 & $0,0 \%$ & ns \\
\hline Abort (8.Wo) nach dem Unfall & 0 & $0,0 \%$ & 1 & $50,0 \%$ & ns \\
\hline Frühgeburt nach dem Unfall (34. Wo) & 1 & $0,5 \%$ & 0 & $0,0 \%$ & ns \\
\hline \multicolumn{6}{|l|}{ Trainingszustand während der Schwangerschaft } \\
\hline ausgezeichnet & 227 & $12,6 \%$ & 3 & $18,8 \%$ & ns \\
\hline sehr gut & 359 & $19,9 \%$ & 2 & $12,5 \%$ & ns \\
\hline gut & 597 & $33,2 \%$ & 7 & $43,8 \%$ & ns \\
\hline mäßig & 424 & $23,6 \%$ & 3 & $18,8 \%$ & ns \\
\hline nicht besonders & 193 & $10,7 \%$ & 1 & $6,3 \%$ & ns \\
\hline \multicolumn{6}{|l|}{$\begin{array}{l}\text { Sportarten während der Schwangerschaft außer } \\
\text { Reiten }\end{array}$} \\
\hline Ausdauersportarten & 685 & $38,1 \%$ & 9 & $56,3 \%$ & ns \\
\hline Gymnastische Sportarten & 201 & $11,2 \%$ & 2 & $12,5 \%$ & ns \\
\hline Spielsportarten & 35 & $1,9 \%$ & 0 & $0,0 \%$ & ns \\
\hline Kraftbetonte Sportarten & 58 & $3,2 \%$ & 0 & $0,0 \%$ & ns \\
\hline Fun- und Risikosportarten & 85 & $4,7 \%$ & 1 & $6,3 \%$ & ns \\
\hline kein Sport & 890 & $49,4 \%$ & 6 & $37,5 \%$ & ns \\
\hline zwei Sportarten & 148 & $8,2 \%$ & 2 & $12,5 \%$ & ns \\
\hline drei Sportarten & 6 & $0,0 \%$ & 0 & $0,0 \%$ & ns \\
\hline & & & & & \\
\hline
\end{tabular}


Schwangerschaften mit Beendigung bis $24+6$ vs. Beendigung ab $25+0$

\begin{tabular}{|c|c|c|c|c|c|}
\hline & $\begin{array}{c}\text { Alle } \\
\text { Teilnehmeri } \\
\text { nnen, ET ab } \\
25 \text { SSW }\end{array}$ & & $\begin{array}{c}\text { Aborte und } \\
\text { Fehlgeburten bis } \\
24+6\end{array}$ & & \\
\hline$n=$ & 1800 & & 16 & & \\
\hline & & & & & Signifikanz \\
\hline \multicolumn{6}{|c|}{$\begin{array}{l}\text { Erkrankung vor oder während der } \\
\text { Schwangerschaft }\end{array}$} \\
\hline \multicolumn{6}{|c|}{ Bluthochdruck } \\
\hline nein & 1711 & $95,1 \%$ & 16 & $100,0 \%$ & $\mathrm{~s}$ \\
\hline vor der Schwangerschaft & 12 & $0,7 \%$ & 0 & $0,0 \%$ & ns \\
\hline während der Schwangerschaft & 77 & $4,3 \%$ & 0 & $0,0 \%$ & ns \\
\hline keine Angabe & 0 & $0,0 \%$ & 0 & $0,0 \%$ & \\
\hline \multicolumn{6}{|l|}{ Diabetes mellitus } \\
\hline nein & 1775 & $98,6 \%$ & 16 & $100,0 \%$ & $\mathrm{~s}$ \\
\hline vor der Schwangerschaft & 3 & $0,2 \%$ & 0 & $0,0 \%$ & ns \\
\hline während der Schwangerschaft & 22 & $1,2 \%$ & 0 & $0,0 \%$ & ns \\
\hline \multicolumn{6}{|l|}{ Herzkrankheit } \\
\hline nein & 1792 & $99,6 \%$ & 16 & $100,0 \%$ & $\mathrm{~s}$ \\
\hline vor der Schwangerschaft & 6 & $0,3 \%$ & 0 & $0,0 \%$ & ns \\
\hline während der Schwangerschaft & 2 & $0,1 \%$ & 0 & $0,0 \%$ & ns \\
\hline \multicolumn{6}{|l|}{ Asthma } \\
\hline nein & 1728 & $96,0 \%$ & 16 & $100,0 \%$ & s \\
\hline vor der Schwangerschaft & 59 & $3,3 \%$ & 0 & $0,0 \%$ & ns \\
\hline während der Schwangerschaft & 13 & $0,7 \%$ & 0 & $0,0 \%$ & ns \\
\hline \multicolumn{6}{|l|}{ Bronchitis } \\
\hline nein & 1713 & $95,2 \%$ & 15 & $93,8 \%$ & ns \\
\hline vor der Schwangerschaft & 41 & $2,3 \%$ & 1 & $6,3 \%$ & ns \\
\hline während der Schwangerschaft & 46 & $2,6 \%$ & 0 & $0,0 \%$ & ns \\
\hline \multicolumn{6}{|c|}{ Krampfadern, off. Bein, Venenthrombose } \\
\hline nein & 1685 & $93,6 \%$ & 15 & $93,8 \%$ & ns \\
\hline vor der Schwangerschaft & 18 & $1,0 \%$ & 1 & $6,3 \%$ & ns \\
\hline während der Schwangerschaft & 97 & $5,4 \%$ & 0 & $0,0 \%$ & ns \\
\hline \multicolumn{6}{|l|}{ Hypothyreose } \\
\hline nein & 1721 & $95,6 \%$ & 15 & $93,8 \%$ & ns \\
\hline vor der Schwangerschaft & 65 & $3,6 \%$ & 1 & $6,3 \%$ & ns \\
\hline während der Schwangerschaft & 14 & $0,8 \%$ & 0 & $0,0 \%$ & ns \\
\hline \multicolumn{6}{|l|}{ Hyperthyreose } \\
\hline nein & 1779 & $98,8 \%$ & 16 & $100,0 \%$ & $s$ \\
\hline vor der Schwangerschaft & 13 & $0,7 \%$ & 0 & $0,0 \%$ & ns \\
\hline während der Schwangerschaft & 8 & $0,4 \%$ & 0 & $0,0 \%$ & ns \\
\hline & & & & & \\
\hline
\end{tabular}




\section{Reiten in der Schwangerschaft}

Aborte

Schwangerschaften mit Beendigung bis $24+6$ vs. Beendigung ab $25+0$

\begin{tabular}{|c|c|c|c|c|c|}
\hline & $\begin{array}{c}\text { Alle } \\
\text { Teilnehmeri } \\
\text { nnen, ET ab } \\
25 \text { SSW }\end{array}$ & & $\begin{array}{l}\text { Aborte und } \\
\text { Fehlgeburten bis } \\
24+6\end{array}$ & & \\
\hline$n=$ & 1800 & & 16 & & \\
\hline & & & & & Signifikanz \\
\hline \multicolumn{6}{|l|}{ Rückenschmerzen } \\
\hline nein & 1111 & $61,7 \%$ & 12 & $75,0 \%$ & ns \\
\hline vor der Schwangerschaft & 202 & $11,2 \%$ & 3 & $18,8 \%$ & ns \\
\hline während der Schwangerschaft & 487 & $27,1 \%$ & 1 & $6,3 \%$ & ns \\
\hline \multicolumn{6}{|l|}{ Karies } \\
\hline nein & 1529 & $84,9 \%$ & 14 & $87,5 \%$ & ns \\
\hline vor der Schwangerschaft & 178 & $9,9 \%$ & 1 & $6,3 \%$ & ns \\
\hline während der Schwangerschaft & 93 & $5,2 \%$ & 1 & $6,3 \%$ & ns \\
\hline \multicolumn{6}{|l|}{ Parodontitis } \\
\hline nein & 1377 & $76,5 \%$ & 15 & $93,8 \%$ & $\mathrm{~s}$ \\
\hline vor der Schwangerschaft & 69 & $3,8 \%$ & 1 & $6,3 \%$ & ns \\
\hline während der Schwangerschaft & 354 & $19,7 \%$ & 0 & $0,0 \%$ & ns \\
\hline \multicolumn{6}{|l|}{ Unfallfolgen } \\
\hline nein & 1747 & $97,1 \%$ & 14 & $87,5 \%$ & ns \\
\hline vor der Schwangerschaft & 20 & $1,1 \%$ & 1 & $6,3 \%$ & ns \\
\hline während der Schwangerschaft & 0 & $0,0 \%$ & 0 & $0,0 \%$ & \\
\hline \multicolumn{6}{|l|}{ Sonstiges } \\
\hline nein & 1556 & $86,4 \%$ & 15 & $93,8 \%$ & ns \\
\hline vor der Schwangerschaft & 62 & $3,4 \%$ & 1 & $6,3 \%$ & ns \\
\hline während der Schwangerschaft & 166 & $9,2 \%$ & 0 & $0,0 \%$ & ns \\
\hline fehlerhafte Daten & 5 & $0,3 \%$ & 2 & $12,5 \%$ & ns \\
\hline fehlerhafte Daten & 13 & $0,7 \%$ & 0 & $0,0 \%$ & ns \\
\hline \multicolumn{6}{|l|}{ Komplikationen während der Schwangerschaft } \\
\hline ja & 901 & $50,1 \%$ & 14 & $87,5 \%$ & $\mathrm{~s}$ \\
\hline nein & 898 & $49,9 \%$ & 2 & $12,5 \%$ & ns \\
\hline \multicolumn{6}{|l|}{$\begin{array}{l}\text { Komplikationen während der Schwangerschaft, } \\
\text { und zwar }\end{array}$} \\
\hline \multicolumn{6}{|l|}{ Scheiden- oder Harnwegsinfekt } \\
\hline nein & 1670 & $92,8 \%$ & 16 & $100,0 \%$ & $\mathrm{~s}$ \\
\hline während der aktiven Reitzeit bis 1 Woche danach & 87 & $4,8 \%$ & 0 & $0,0 \%$ & ns \\
\hline später & 45 & $2,5 \%$ & 0 & $0,0 \%$ & \\
\hline \multicolumn{6}{|l|}{ Vorzeitige Wehentätigkeit } \\
\hline nein & 1598 & $88,8 \%$ & 15 & $93,8 \%$ & ns \\
\hline während der aktiven Reitzeit bis 1 Woche danach & 68 & $3,8 \%$ & 2 & $12,5 \%$ & ns \\
\hline später & 136 & $7,6 \%$ & 1 & $6,3 \%$ & ns \\
\hline
\end{tabular}




\begin{tabular}{|c|c|c|c|c|c|}
\hline & $\begin{array}{c}\text { Alle } \\
\text { Teilnehmeri } \\
\text { nnen, ET ab } \\
25 \text { SSW }\end{array}$ & & $\begin{array}{l}\text { Aborte und } \\
\text { Fehlgeburten bis } \\
24+6\end{array}$ & & \\
\hline$n=$ & 1800 & & 16 & & \\
\hline & & & & & Signifikanz \\
\hline \multicolumn{6}{|l|}{ Vorzeitiger Blasensprung } \\
\hline nein & 1706 & $94,8 \%$ & 16 & $100,0 \%$ & $\mathrm{~s}$ \\
\hline während der aktiven Reitzeit bis 1 Woche danach & 18 & $1,0 \%$ & 1 & $6,3 \%$ & ns \\
\hline später & 78 & $4,3 \%$ & 0 & $0,0 \%$ & ns \\
\hline \multicolumn{6}{|l|}{ Zervixinsuffizienz } \\
\hline nein & 1693 & $94,1 \%$ & 16 & $100,0 \%$ & ns \\
\hline während der aktiven Reitzeit bis 1 Woche danach & 43 & $2,4 \%$ & 1 & $6,3 \%$ & ns \\
\hline später & 66 & $3,7 \%$ & 0 & $0,0 \%$ & ns \\
\hline \multicolumn{6}{|l|}{ Amnioninfektionssyndrom } \\
\hline nein & 1798 & $99,9 \%$ & 16 & $100,0 \%$ & ns \\
\hline während der aktiven Reitzeit bis 1 Woche danach & 0 & $0,0 \%$ & 0 & $0,0 \%$ & ns \\
\hline später & 4 & $0,2 \%$ & 0 & $0,0 \%$ & ns \\
\hline \multicolumn{6}{|l|}{ Ablösung der Plazenta } \\
\hline nein & 1786 & $99,2 \%$ & 16 & $100,0 \%$ & \\
\hline während der aktiven Reitzeit bis 1 Woche danach & 3 & $0,2 \%$ & 1 & $6,3 \%$ & ns \\
\hline später & 13 & $0,7 \%$ & 0 & $0,0 \%$ & ns \\
\hline \multicolumn{6}{|l|}{ Plazenta-Insuffizienz } \\
\hline nein & 1762 & $97,9 \%$ & 16 & $100,0 \%$ & ns \\
\hline während der aktiven Reitzeit bis 1 Woche danach & 14 & $0,8 \%$ & 0 & $0,0 \%$ & ns \\
\hline später & 26 & $1,4 \%$ & 1 & $6,3 \%$ & ns \\
\hline \multicolumn{6}{|l|}{ Bluthochdruck } \\
\hline nein & 1732 & $96,2 \%$ & 16 & $100,0 \%$ & ns \\
\hline während der aktiven Reitzeit bis 1 Woche danach & 18 & $1,0 \%$ & 0 & $0,0 \%$ & ns \\
\hline später & 52 & $2,9 \%$ & 0 & $0,0 \%$ & ns \\
\hline \multicolumn{6}{|l|}{ Präeklampsie / Gestose } \\
\hline nein & 1762 & $97,9 \%$ & 16 & $100,0 \%$ & ns \\
\hline während der aktiven Reitzeit bis 1 Woche danach & 2 & $0,1 \%$ & 0 & $0,0 \%$ & ns \\
\hline später & 38 & $2,1 \%$ & 0 & $0,0 \%$ & ns \\
\hline \multicolumn{6}{|l|}{ Eklampsie / HELLP-Syndrom } \\
\hline nein & 1793 & $99,6 \%$ & 16 & $100,0 \%$ & ns \\
\hline während der aktiven Reitzeit bis 1 Woche danach & 2 & $0,1 \%$ & 0 & $0,0 \%$ & ns \\
\hline später & 7 & $0,4 \%$ & 0 & $0,0 \%$ & ns \\
\hline \multicolumn{6}{|l|}{ Schwangerschaftsdiabetes } \\
\hline nein & 1766 & $98,1 \%$ & 16 & $100,0 \%$ & ns \\
\hline während der aktiven Reitzeit bis 1 Woche danach & 14 & $0,8 \%$ & 0 & $0,0 \%$ & ns \\
\hline später & 22 & $1,2 \%$ & 0 & $0,0 \%$ & ns \\
\hline
\end{tabular}


Schwangerschaften mit Beendigung bis $24+6$ vs. Beendigung ab $25+0$

\begin{tabular}{|c|c|c|c|c|c|}
\hline & $\begin{array}{c}\text { Alle } \\
\text { Teilnehmeri } \\
\text { nnen, ET ab } \\
25 \text { SSW }\end{array}$ & & $\begin{array}{l}\text { Aborte und } \\
\text { Fehlgeburten bis } \\
24+6\end{array}$ & & \\
\hline$n=$ & 1800 & & 16 & & \\
\hline & & & & & Signifikanz \\
\hline \multicolumn{6}{|l|}{ Hydramnion } \\
\hline nein & 1793 & $99,6 \%$ & 16 & $100,0 \%$ & ns \\
\hline während der aktiven Reitzeit bis 1 Woche danach & 3 & $0,2 \%$ & 1 & $6,3 \%$ & ns \\
\hline später & 6 & $0,3 \%$ & 0 & $0,0 \%$ & ns \\
\hline \multicolumn{6}{|l|}{ Fehlbildungen } \\
\hline nein & 1797 & $99,8 \%$ & 16 & $100,0 \%$ & ns \\
\hline während der aktiven Reitzeit bis 1 Woche danach & 1 & $0,1 \%$ & 0 & $0,0 \%$ & ns \\
\hline später & 4 & $0,2 \%$ & 0 & $0,0 \%$ & ns \\
\hline \multicolumn{6}{|l|}{ Uterusmyom } \\
\hline nein & 1784 & $99,1 \%$ & 16 & $100,0 \%$ & ns \\
\hline während der aktiven Reitzeit bis 1 Woche danach & 5 & $0,3 \%$ & 0 & $0,0 \%$ & ns \\
\hline später & 13 & $0,7 \%$ & 0 & $0,0 \%$ & ns \\
\hline \multicolumn{6}{|l|}{ Blutungen } \\
\hline nein & 1687 & $93,7 \%$ & 8 & $50,0 \%$ & ns \\
\hline während der aktiven Reitzeit bis 1 Woche danach & 86 & $4,8 \%$ & 8 & $50,0 \%$ & ns \\
\hline später & 29 & $1,6 \%$ & 2 & $12,5 \%$ & ns \\
\hline \multicolumn{6}{|l|}{ Fieber } \\
\hline nein & 1767 & $98,2 \%$ & 16 & $100,0 \%$ & ns \\
\hline während der aktiven Reitzeit bis 1 Woche danach & 20 & $1,1 \%$ & 0 & $0,0 \%$ & ns \\
\hline später & 15 & $0,8 \%$ & 1 & $6,3 \%$ & ns \\
\hline \multicolumn{6}{|l|}{ Blutgruppen-Unverträglichkeit } \\
\hline nein & 1779 & $98,8 \%$ & 16 & $100,0 \%$ & ns \\
\hline während der aktiven Reitzeit bis 1 Woche danach & 15 & $0,8 \%$ & 0 & $0,0 \%$ & ns \\
\hline später & 8 & $0,4 \%$ & 0 & $0,0 \%$ & ns \\
\hline \multicolumn{6}{|l|}{ Toxoplasmose } \\
\hline nein & 1799 & $99,9 \%$ & 16 & $100,0 \%$ & ns \\
\hline während der aktiven Reitzeit bis 1 Woche danach & 3 & $0,2 \%$ & 0 & $0,0 \%$ & ns \\
\hline später & 0 & $0,0 \%$ & 0 & $0,0 \%$ & \\
\hline \multicolumn{6}{|l|}{ Sonstige Infektionskrankheiten } \\
\hline nein & 1750 & $97,2 \%$ & 15 & $93,7 \%$ & ns \\
\hline während der aktiven Reitzeit bis 1 Woche danach & 30 & $1,7 \%$ & 0 & $0,0 \%$ & ns \\
\hline später & 22 & $1,2 \%$ & 1 & $6,3 \%$ & ns \\
\hline
\end{tabular}


Schwangerschaften mit Beendigung bis $24+6$ vs. Beendigung ab $25+0$

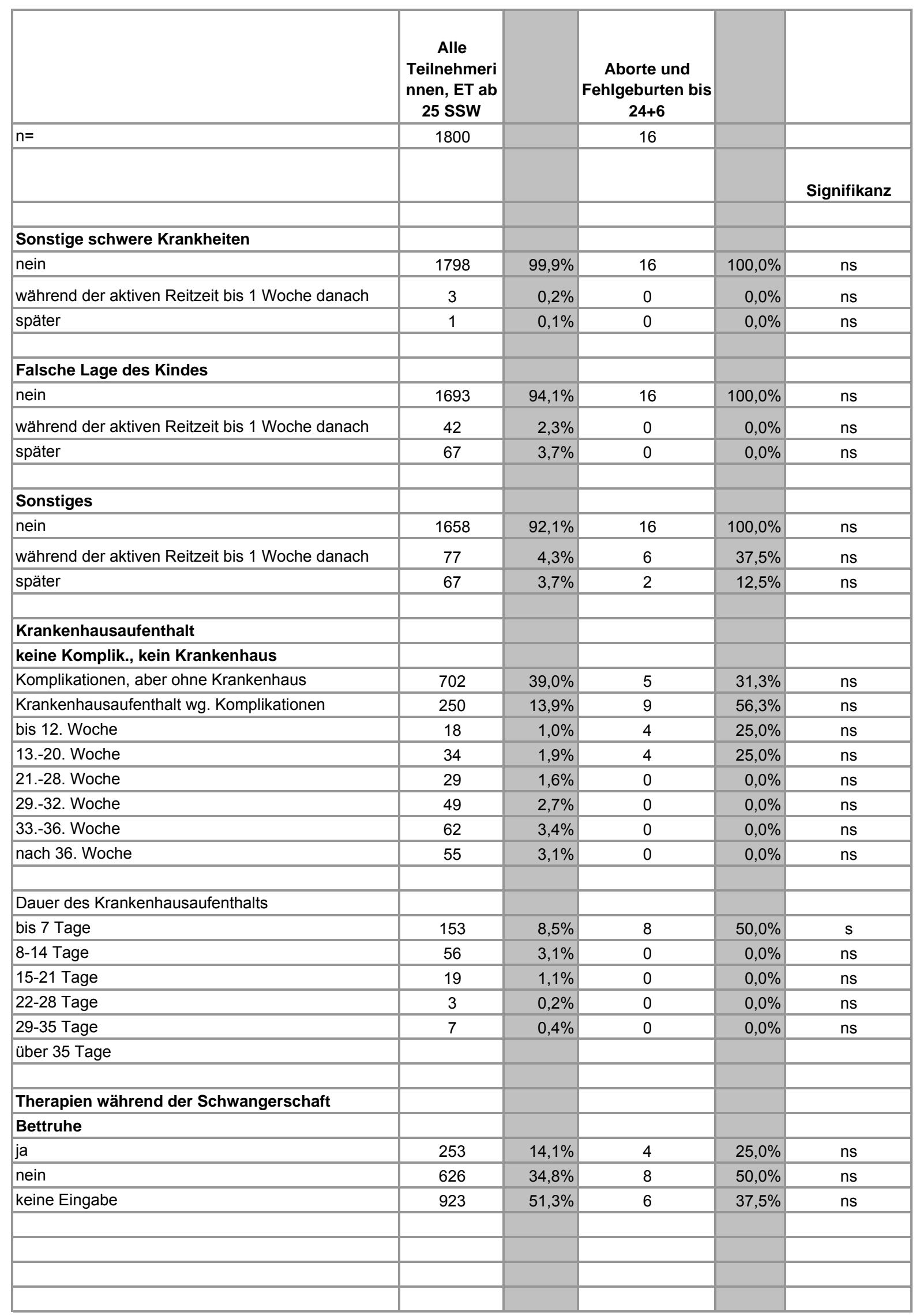


Schwangerschaften mit Beendigung bis $24+6$ vs. Beendigung ab $25+0$

\begin{tabular}{|c|c|c|c|c|c|}
\hline & $\begin{array}{c}\text { Alle } \\
\text { Teilnehmeri } \\
\text { nnen, ET ab } \\
25 \text { SSW }\end{array}$ & & $\begin{array}{l}\text { Aborte und } \\
\text { Fehlgeburten bis } \\
24+6\end{array}$ & & \\
\hline$n=$ & 1800 & & 16 & & \\
\hline & & & & & Signifikanz \\
\hline \multicolumn{6}{|l|}{ Antibiotika } \\
\hline ja & 90 & $5,0 \%$ & 2 & $12,5 \%$ & ns \\
\hline nein & 782 & $43,4 \%$ & 10 & $62,5 \%$ & ns \\
\hline keine Eingabe & 930 & $51,7 \%$ & 6 & $37,5 \%$ & ns \\
\hline \multicolumn{6}{|l|}{ Antimykotika } \\
\hline ja & 91 & $5,1 \%$ & 0 & $0,0 \%$ & ns \\
\hline nein & 779 & $43,3 \%$ & 12 & $75,0 \%$ & $\mathrm{~s}$ \\
\hline keine Eingabe & 932 & $51,8 \%$ & 6 & $37,5 \%$ & ns \\
\hline \multicolumn{6}{|c|}{ Wehenhemmung } \\
\hline ja & 115 & $6,4 \%$ & 2 & $12,5 \%$ & ns \\
\hline nein & 756 & $42,0 \%$ & 11 & $68,8 \%$ & $\mathrm{~s}$ \\
\hline keine Eingabe & 931 & $51,7 \%$ & 5 & $31,3 \%$ & ns \\
\hline \multicolumn{6}{|l|}{ Magnesium } \\
\hline ja & 269 & $14,9 \%$ & 3 & $18,8 \%$ & ns \\
\hline nein & 607 & $33,7 \%$ & 10 & $62,5 \%$ & $\mathrm{~s}$ \\
\hline keine Eingabe & 926 & $51,4 \%$ & 5 & $31,3 \%$ & ns \\
\hline \multicolumn{6}{|c|}{ Medikamente zur Lungenreifung } \\
\hline ja & 81 & $4,5 \%$ & 0 & $0,0 \%$ & ns \\
\hline nein & 790 & $43,9 \%$ & 12 & $75,0 \%$ & $\mathrm{~s}$ \\
\hline keine Eingabe & 931 & $51,7 \%$ & 6 & $37,5 \%$ & ns \\
\hline \multicolumn{6}{|c|}{ Blutdrucksenkung } \\
\hline ja & 41 & $2,3 \%$ & 0 & $0,0 \%$ & ns \\
\hline nein & 829 & $46,1 \%$ & 12 & $75,0 \%$ & $\mathrm{~s}$ \\
\hline keine Eingabe & 932 & $51,8 \%$ & 6 & $37,5 \%$ & ns \\
\hline \multicolumn{6}{|c|}{ Behandlung des Diabetes } \\
\hline ja & 24 & $1,3 \%$ & 0 & $0,0 \%$ & ns \\
\hline nein & 845 & $46,9 \%$ & 12 & $75,0 \%$ & $\mathrm{~s}$ \\
\hline keine Eingabe & 933 & $51,8 \%$ & 6 & $37,5 \%$ & ns \\
\hline \multicolumn{6}{|l|}{ Cerclage } \\
\hline ja & 26 & $1,4 \%$ & 0 & $0,0 \%$ & ns \\
\hline nein & 840 & $46,7 \%$ & 12 & $75,0 \%$ & $\mathrm{~s}$ \\
\hline keine Eingabe & 936 & $52,0 \%$ & 6 & $37,5 \%$ & ns \\
\hline \multicolumn{6}{|c|}{ Sonstige Maßnahme } \\
\hline ja & 1628 & $90,4 \%$ & 11 & $68,8 \%$ & ns \\
\hline nein & 15 & $0,8 \%$ & 0 & $0,0 \%$ & ns \\
\hline keine Eingabe & 159 & $8,8 \%$ & 7 & $43,8 \%$ & $\mathrm{~s}$ \\
\hline
\end{tabular}


Schwangerschaften mit Beendigung bis $24+6$ vs. Beendigung ab $25+0$

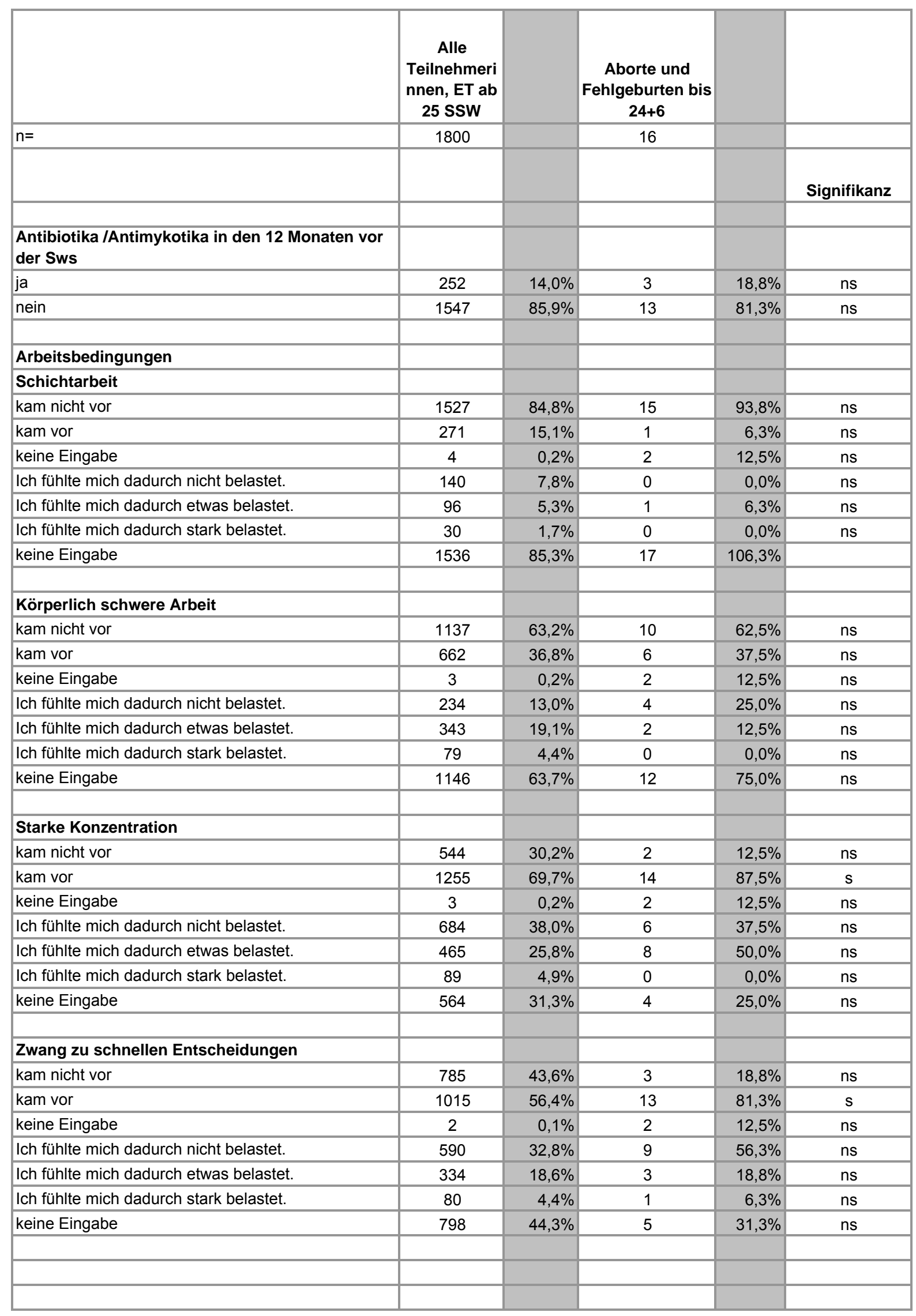


Schwangerschaften mit Beendigung bis $24+6$ vs. Beendigung ab $25+0$

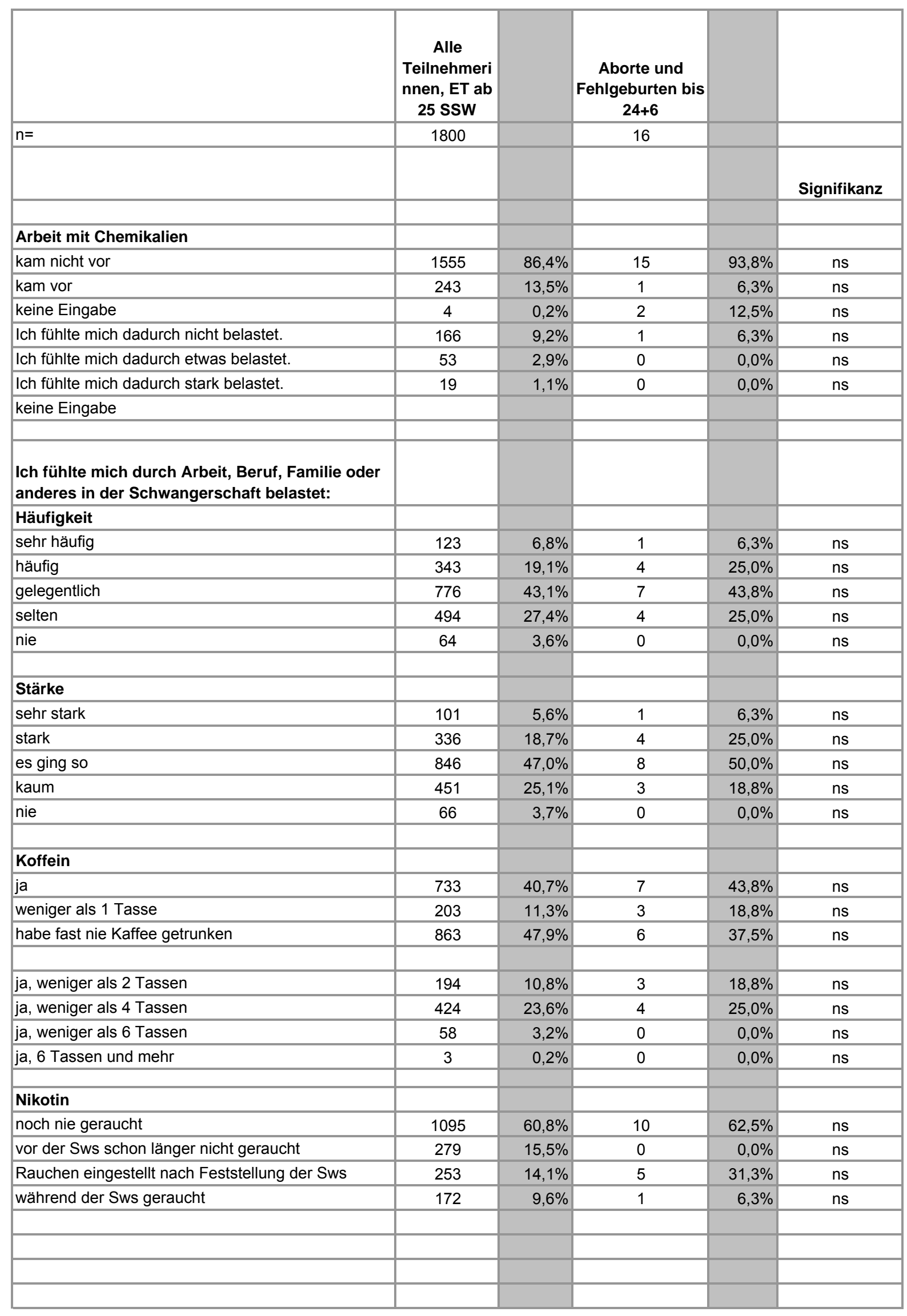


Schwangerschaften mit Beendigung bis $24+6$ vs. Beendigung ab $25+0$

\begin{tabular}{|c|c|c|c|c|c|}
\hline & $\begin{array}{c}\text { Alle } \\
\text { Teilnehmeri } \\
\text { nnen, ET ab } \\
25 \text { SSW }\end{array}$ & & $\begin{array}{c}\text { Aborte und } \\
\text { Fehlgeburten bis } \\
24+6\end{array}$ & & \\
\hline$n=$ & 1800 & & 16 & & \\
\hline & & & & & Signifikanz \\
\hline \multicolumn{6}{|c|}{ Zahl der Zigaretten pro Tag vor der Sws } \\
\hline keine Zigaretteen & 1555 & $86,4 \%$ & 11 & $68,8 \%$ & ns \\
\hline 1-2 Zigaretten & 20 & $1,1 \%$ & 1 & $6,3 \%$ & ns \\
\hline 3-4 Zigaretten & 23 & $1,3 \%$ & 0 & $0,0 \%$ & ns \\
\hline 5-6 Zigaretten & 38 & $2,1 \%$ & 0 & $0,0 \%$ & ns \\
\hline 7-8 Zigaretten & 10 & $0,6 \%$ & 2 & $12,5 \%$ & ns \\
\hline 9-10 Zigaretten & 36 & $2,0 \%$ & 0 & $0,0 \%$ & ns \\
\hline mehr als 10 Zigaretten & 118 & $6,6 \%$ & 2 & $12,5 \%$ & ns \\
\hline \multicolumn{6}{|c|}{ Zahl der Zigaretten pro Tag während der Sws } \\
\hline keine Zigaretteen & 1630 & $90,6 \%$ & 15 & $93,8 \%$ & ns \\
\hline 1-2 Zigaretten & 35 & $1,9 \%$ & 1 & $6,3 \%$ & ns \\
\hline 3-4 Zigaretten & 30 & $1,7 \%$ & 0 & $0,0 \%$ & ns \\
\hline 5-6 Zigaretten & 46 & $2,6 \%$ & 0 & $0,0 \%$ & ns \\
\hline 7-8 Zigaretten & 7 & $0,4 \%$ & 0 & $0,0 \%$ & ns \\
\hline 9-10 Zigaretten & 31 & $1,7 \%$ & 0 & $0,0 \%$ & ns \\
\hline mehr als 10 Zigaretten & 21 & $1,2 \%$ & 0 & $0,0 \%$ & ns \\
\hline \multicolumn{6}{|l|}{ Passivrauchen } \\
\hline ja, bei der Arbeit & 215 & $11,9 \%$ & 2 & $12,5 \%$ & ns \\
\hline ja, zu Hause & 193 & $10,7 \%$ & 2 & $12,5 \%$ & ns \\
\hline ja, an anderen Orten & 392 & $21,8 \%$ & 3 & $18,8 \%$ & ns \\
\hline nein & 1162 & $64,6 \%$ & 12 & $75,0 \%$ & ns \\
\hline an 2 Orten & 150 & $8,3 \%$ & 3 & $18,8 \%$ & ns \\
\hline an 3 Orten & 26 & $1,4 \%$ & 0 & $0,0 \%$ & ns \\
\hline \multicolumn{6}{|l|}{ Wein, Sekt, Obstwein } \\
\hline ja & 24 & $1,3 \%$ & 0 & $0,0 \%$ & ns \\
\hline weniger als 1 Glas & 156 & $8,7 \%$ & 1 & $6,3 \%$ & ns \\
\hline habe fast nie Wein etc. getrunken & 1620 & $90,0 \%$ & 15 & $93,8 \%$ & ns \\
\hline ja, 1 Glas pro Tag & 17 & $0,9 \%$ & 0 & $0,0 \%$ & ns \\
\hline ja, 2 Gläser pro Tag & 1 & $0,1 \%$ & 0 & $0,0 \%$ & ns \\
\hline ja, mehr als 2 Gläser pro Tag & 0 & $0,0 \%$ & 0 & $0,0 \%$ & \\
\hline \multicolumn{6}{|l|}{ Bier } \\
\hline ja & 25 & $1,4 \%$ & 0 & $0,0 \%$ & ns \\
\hline weniger als 1 Glas & 76 & $4,2 \%$ & 1 & $6,3 \%$ & ns \\
\hline habe fast nie Bier getrunken & 1699 & $94,4 \%$ & 15 & $93,8 \%$ & ns \\
\hline ja, 1 Glas pro Tag & 7 & $0,4 \%$ & 0 & $0,0 \%$ & ns \\
\hline ja, 2 Gläser pro Tag & 5 & $0,3 \%$ & 0 & $0,0 \%$ & ns \\
\hline ja, mehr als 2 Gläser pro Tag & 0 & $0,0 \%$ & 0 & $0,0 \%$ & \\
\hline & & & & & \\
\hline & & & & & \\
\hline
\end{tabular}


Schwangerschaften mit Beendigung bis $24+6$ vs. Beendigung ab $25+0$

\begin{tabular}{|c|c|c|c|c|c|}
\hline & $\begin{array}{c}\text { Alle } \\
\text { Teilnehmeri } \\
\text { nnen, ET ab } \\
25 \text { SSW }\end{array}$ & & $\begin{array}{c}\text { Aborte und } \\
\text { Fehlgeburten bis } \\
24+6\end{array}$ & & \\
\hline$n=$ & 1800 & & 16 & & \\
\hline & & & & & Signifikanz \\
\hline \multicolumn{6}{|l|}{ Spirituosen } \\
\hline ja, gelegentlich & 6 & $0,3 \%$ & 0 & $0,0 \%$ & ns \\
\hline ja, selten & 74 & $4,1 \%$ & 0 & $0,0 \%$ & ns \\
\hline nein, niemals & 1720 & $95,6 \%$ & 16 & $100,0 \%$ & $\mathrm{~s}$ \\
\hline \multicolumn{6}{|l|}{ Kinderwunschbehandlung } \\
\hline ja & 77 & $4,3 \%$ & 0 & $0,0 \%$ & ns \\
\hline nein & 1723 & $95,7 \%$ & 16 & $100,0 \%$ & $\mathrm{~s}$ \\
\hline \multicolumn{6}{|c|}{$\begin{array}{l}\text { Fehlbildungen in der Familie der Mutter (1./2. } \\
\text { Grades) }\end{array}$} \\
\hline ja & 63 & $3,5 \%$ & 3 & $18,8 \%$ & ns \\
\hline nein & 1737 & $96,5 \%$ & 13 & $81,3 \%$ & ns \\
\hline \multicolumn{6}{|l|}{ ENTBINDUNG } \\
\hline \multicolumn{6}{|l|}{ Dauer der Schwangerschaft } \\
\hline Ende in Sws-Woche - Mittelwert & 39,53 & & 11,6 & & $\mathrm{~s}$ \\
\hline bis 12 . Woche & 0 & $0,0 \%$ & 9 & $56,3 \%$ & ns \\
\hline 13.-16. Woche & 0 & $0,0 \%$ & 4 & $25,0 \%$ & ns \\
\hline 17.-20. Woche & 0 & $0,0 \%$ & 2 & $12,5 \%$ & ns \\
\hline 21.-24. Woche & 0 & $0,0 \%$ & 1 & $6,3 \%$ & ns \\
\hline gesamt vor 25 . Woche & 0 & $0,0 \%$ & 16 & $100,0 \%$ & \\
\hline gesamt vor 25 . Woche ohne 1 . Trimenon & 0 & $0,0 \%$ & 7 & $43,8 \%$ & \\
\hline 25.-26. Woche & 3 & $0,2 \%$ & 0 & $0,0 \%$ & ns \\
\hline 27.-31. Woche & 7 & $0,4 \%$ & 0 & $0,0 \%$ & ns \\
\hline 32.-36. Woche & 111 & $6,2 \%$ & 0 & $0,0 \%$ & ns \\
\hline 37.-41. Woche & 1470 & $81,7 \%$ & 0 & $0,0 \%$ & ns \\
\hline nach 41. Woche & 205 & $11,4 \%$ & 0 & $0,0 \%$ & ns \\
\hline gesamt ab 37. Woche & 1675 & $93,1 \%$ & 0 & $0,0 \%$ & ns \\
\hline \multicolumn{6}{|l|}{ Wie viele Kinder? } \\
\hline Einling & 1777 & $98,7 \%$ & 12 & $75,0 \%$ & $\mathrm{~s}$ \\
\hline Mehrlingsgeburt & 23 & $1,3 \%$ & 2 & $12,5 \%$ & ns \\
\hline \multicolumn{6}{|l|}{ Gewicht und Größe des Kindes } \\
\hline \multicolumn{6}{|l|}{ Körpergewicht in g } \\
\hline Mittelwert & 3415,920653 & & \#DIV/0! & & \\
\hline bis $600 \mathrm{~g}$ & 1 & $0,1 \%$ & 0 & $0,0 \%$ & ns \\
\hline $601-1000 \mathrm{~g}$ & 1 & $0,1 \%$ & 0 & $0,0 \%$ & ns \\
\hline $1001-1500 \mathrm{~g}$ & 3 & $0,2 \%$ & 0 & $0,0 \%$ & ns \\
\hline $1501-2000 \mathrm{~g}$ & 9 & $0,5 \%$ & 0 & $0,0 \%$ & ns \\
\hline $2001-2500 \mathrm{~g}$ & 66 & $3,7 \%$ & 0 & $0,0 \%$ & ns \\
\hline $2501-3000 \mathrm{~g}$ & 263 & $14,6 \%$ & 0 & $0,0 \%$ & ns \\
\hline
\end{tabular}


Schwangerschaften mit Beendigung bis $24+6$ vs. Beendigung ab $25+0$

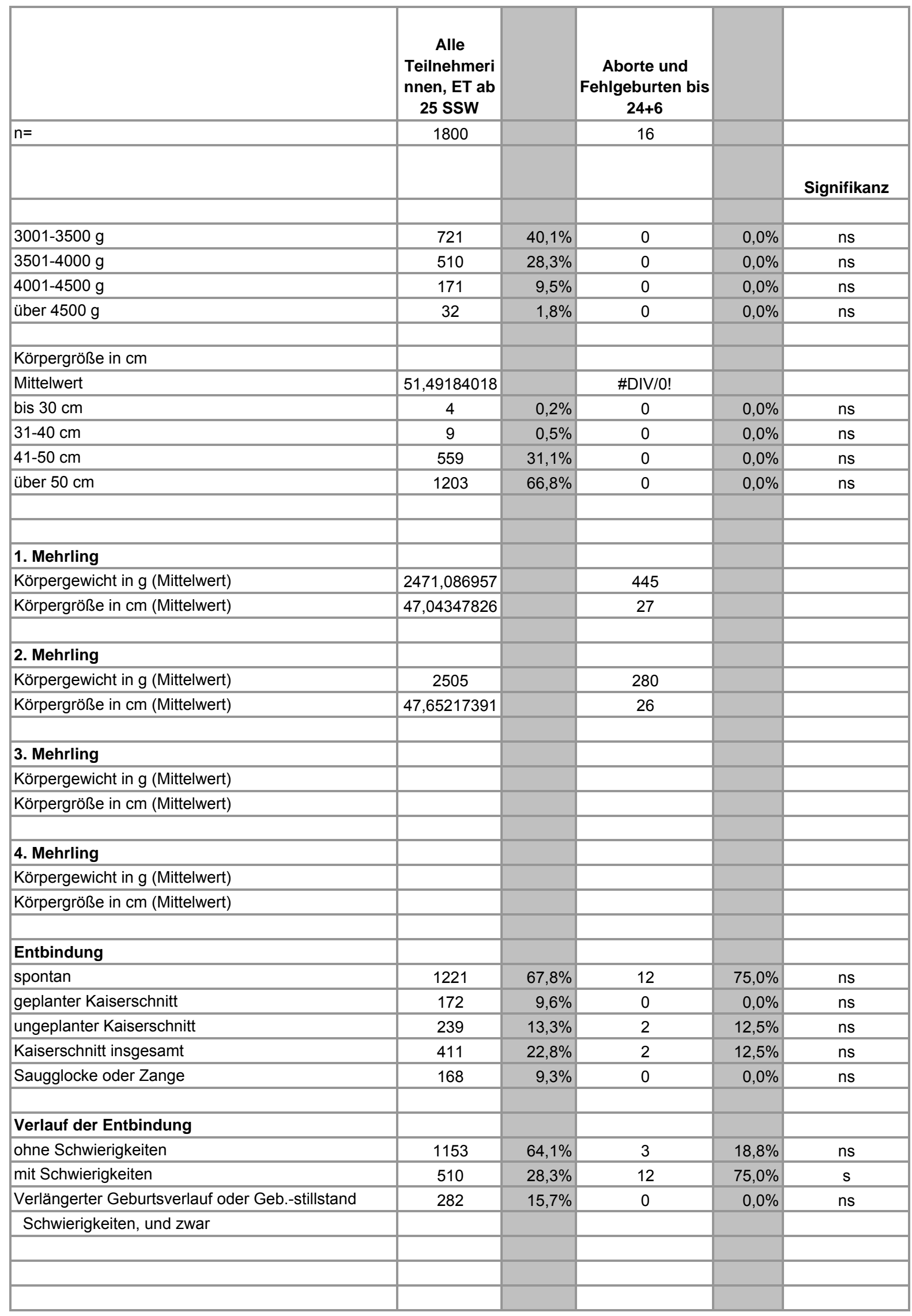


Schwangerschaften mit Beendigung bis $24+6$ vs. Beendigung ab $25+0$

\begin{tabular}{|c|c|c|c|c|c|}
\hline & $\begin{array}{c}\text { Alle } \\
\text { Teilnehmeri } \\
\text { nnen, ET ab } \\
25 \text { SSW }\end{array}$ & & $\begin{array}{c}\text { Aborte und } \\
\text { Fehlgeburten bis } \\
24+6\end{array}$ & & \\
\hline$n=$ & 1800 & & 16 & & \\
\hline & & & & & Signifikanz \\
\hline \multicolumn{6}{|l|}{ Kind nach der Geburt } \\
\hline gesund geboren & 1701 & $94,5 \%$ & 1 & $6,3 \%$ & ns \\
\hline krank geboren & 98 & $5,4 \%$ & 1 & $6,3 \%$ & ns \\
\hline Totgeburt & 0 & $0,0 \%$ & 14 & $87,5 \%$ & ns \\
\hline \multicolumn{6}{|l|}{ krank geboren, und zwar } \\
\hline \multicolumn{6}{|l|}{ Kind (er) nach einem Jahr } \\
\hline Kind (alle Kinder) gesund & 1741 & $96,7 \%$ & 2 & $12,5 \%$ & ns \\
\hline Kind (bzw. eines der Kinder) krank & 55 & $3,1 \%$ & 3 & $18,8 \%$ & ns \\
\hline Kind (bzw. eines der Kinder) verstorben & 3 & $0,2 \%$ & 10 & $62,5 \%$ & ns \\
\hline \multicolumn{6}{|l|}{ Kind krank, und zwar } \\
\hline \multicolumn{6}{|l|}{ Jahrgang der Entbindung } \\
\hline 2005 & 225 & $12,5 \%$ & 4 & $25,0 \%$ & ns \\
\hline 2004 & 340 & $18,9 \%$ & 4 & $25,0 \%$ & ns \\
\hline 2003 & 225 & $12,5 \%$ & 1 & $6,3 \%$ & ns \\
\hline 2002 & 187 & $10,4 \%$ & 1 & $6,3 \%$ & ns \\
\hline 2001 & 138 & $7,7 \%$ & 1 & $6,3 \%$ & ns \\
\hline 2000 & 124 & $6,9 \%$ & 4 & $25,0 \%$ & ns \\
\hline 1999 & 85 & $4,7 \%$ & 0 & $0,0 \%$ & ns \\
\hline 1998 & 71 & $3,9 \%$ & 0 & $0,0 \%$ & ns \\
\hline 1997 & 88 & $4,9 \%$ & 0 & $0,0 \%$ & ns \\
\hline 1996 & 47 & $2,6 \%$ & 0 & $0,0 \%$ & ns \\
\hline 1995 & 50 & $2,8 \%$ & 1 & $6,3 \%$ & ns \\
\hline 1994 & 34 & $1,9 \%$ & 0 & $0,0 \%$ & ns \\
\hline 1993 & 35 & $1,9 \%$ & 0 & $0,0 \%$ & ns \\
\hline 1992 & 27 & $1,5 \%$ & 0 & $0,0 \%$ & ns \\
\hline 1991 & 21 & $1,2 \%$ & 0 & $0,0 \%$ & ns \\
\hline vor 1991 & 103 & $5,7 \%$ & 0 & $0,0 \%$ & ns \\
\hline
\end{tabular}




\begin{tabular}{|c|c|c|c|c|c|c|c|}
\hline & & $\begin{array}{c}\text { PP } \\
\text { Keine } \\
\text { vorz. } \\
\text { Wehen }\end{array}$ & & $\begin{array}{l}\text { PP mit } \\
\text { vorz. } \\
\text { Wehen }\end{array}$ & & Wurzel S & $\begin{array}{l}\text { Signi- } \\
\text { fikanz }\end{array}$ \\
\hline & $n=$ & 423 & & 126 & & & \\
\hline Alter zu Beginn des SS & 30-34 Jahre & 170 & $40,2 \%$ & 38 & $30,2 \%$ & 2,12 & s \\
\hline BMI & Untergewicht $<20$ & 72 & $17,0 \%$ & 48 & $38,1 \%$ & 4,49 & s \\
\hline BMI & Normalgewicht 20-24,9 & 276 & $65,2 \%$ & 68 & $54,0 \%$ & 2,25 & s \\
\hline BMI & Übergewicht > 25 & 75 & $17,7 \%$ & 10 & $7,9 \%$ & 3,22 & s \\
\hline Gyn-Kompl. Vor der SWS & Summe der Nennungen & 57 & $13,5 \%$ & 33 & $26,2 \%$ & 2,99 & $\mathrm{~s}$ \\
\hline Gyn-Kompl. Vor der SWS & $1 \mathrm{Kompl}$. Angegeben & 40 & $9,5 \%$ & 21 & $16,7 \%$ & 2,00 & s \\
\hline Gyn-Kompl. Vor der SWS & Gesamt mit Komplikationen & 43 & $10,2 \%$ & 24 & $19,0 \%$ & 2,34 & s \\
\hline Schulabschluss & Fachhochschulreife & 40 & $9,5 \%$ & 22 & $17,5 \%$ & 2,18 & s \\
\hline Schulabschluss & $\begin{array}{l}\text { Allg. oder fachgebundene } \\
\text { Hochschulreife }\end{array}$ & 237 & $56,0 \%$ & 55 & $43,7 \%$ & 2,46 & s \\
\hline Reittage pro Woche vor der SWS & 2x pro Woche & 40 & $9,5 \%$ & 6 & $4,8 \%$ & 1,98 & s \\
\hline Reittage pro Woche vor der SWS & täglich & 156 & $36,9 \%$ & 54 & $42,9 \%$ & 1,20 & ns \\
\hline Dressur vor der SWS & insgesamt geritten & 349 & $82,5 \%$ & 116 & $92,1 \%$ & 3,15 & $\mathrm{~s}$ \\
\hline Dressur vor der SWS & leistungsorientiertes Reiten & 156 & $36,9 \%$ & 67 & $53,2 \%$ & 3,24 & s \\
\hline Springen vor der SWS & insgesamt geritten & 214 & $50,6 \%$ & 80 & $63,5 \%$ & 2,62 & s \\
\hline Springen vor der SWS & leistungsorientiertes Reiten & 60 & $14,2 \%$ & 28 & $22,2 \%$ & 1,97 & s \\
\hline Gangpferde vor der SWS & insgesamt geritten & 43 & $10,2 \%$ & 5 & $4,0 \%$ & 2,72 & s \\
\hline $\begin{array}{l}\text { Nach Feststellung der Sws } \\
\text { weitergeritten }\end{array}$ & $\mathrm{Ja}$ & 394 & $93,1 \%$ & 111 & $88,1 \%$ & 1,61 & ns \\
\hline $\begin{array}{l}\text { Nach Feststellung der Sws } \\
\text { weitergeritten }\end{array}$ & $\begin{array}{l}\text { und zwar bis zur } \\
\text { Schwangerschaftswoche }\end{array}$ & 29,06 & & 25,77 & & & ns \\
\hline $\begin{array}{l}\text { Ich habe mit dem Reiten } \\
\text { aufgehört, }\end{array}$ & $\begin{array}{l}\text { Blutungen oder andere } \\
\text { Probleme mit der } \\
\text { Schwangerschaft bekommen }\end{array}$ & 37 & $8,7 \%$ & 30 & $23,8 \%$ & 3,73 & s \\
\hline $\begin{array}{l}\text { Ich habe mit dem Reiten } \\
\text { aufgehört, }\end{array}$ & $\begin{array}{l}\text { andere Person mir das } \\
\text { Aufhören dringend nahe }\end{array}$ & 29 & $6,9 \%$ & 18 & $14,3 \%$ & 2,22 & s \\
\hline $\begin{array}{l}\text { Ich habe mit dem Reiten } \\
\text { aufgehört, }\end{array}$ & $\begin{array}{l}\text { erst als das Reiten } \\
\text { unbequem wurde - allein }\end{array}$ & 164 & $38,8 \%$ & 23 & $18,3 \%$ & 4,91 & s \\
\hline $\begin{array}{l}\text { Ich habe mit dem Reiten } \\
\text { aufgehört, }\end{array}$ & $\begin{array}{l}\text { weil ich vorzeitige Wehen, } \\
\text { etc. bekommen habe - allein }\end{array}$ & 18 & $4,3 \%$ & 14 & $11,1 \%$ & 2,31 & s \\
\hline $\begin{array}{l}\text { Ich habe mit dem Reiten } \\
\text { aufgehört, }\end{array}$ & zwei Gründe angegeben & 100 & $23,6 \%$ & 44 & $34,9 \%$ & 2,39 & s \\
\hline $\begin{array}{l}\text { Trotz Warnzeichen weitergeritten } \\
\text {... }\end{array}$ & 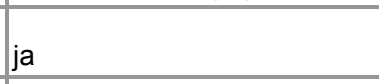 & 27 & $6,4 \%$ & 12 & $9,5 \%$ & 1,09 & ns \\
\hline $\begin{array}{l}\text { Trotz Warnzeichen weitergeritten } \\
\ldots\end{array}$ & nein & 349 & $82,5 \%$ & 93 & $73,8 \%$ & 2,01 & s \\
\hline $\begin{array}{l}\text { Durchschnittliche Reitdauer } \\
\text { während der SWS }\end{array}$ & $1 \mathrm{~h}$ & 347 & $82,0 \%$ & 113 & $89,7 \%$ & 2,32 & s \\
\hline $\begin{array}{l}\text { Durchschnittliche Reitdauer } \\
\text { während der SWS }\end{array}$ & $2-3 \mathrm{~h}$ & 72 & $17,0 \%$ & 13 & $10,3 \%$ & 2,05 & $\mathrm{~s}$ \\
\hline Ausreiten während der SWS & insgesamt geritten & 351 & $83,0 \%$ & 93 & $73,8 \%$ & 2,12 & s \\
\hline Distanzreiten & insgesamt geritten & 56 & $13,2 \%$ & 9 & $7,1 \%$ & 2,16 & $\mathrm{~s}$ \\
\hline Reiten-Rubriken in SWS & $\begin{array}{l}\text { Geländebetont, leichte } \\
\text { Belastung }\end{array}$ & 126 & $29,8 \%$ & 24 & $19,0 \%$ & 2,59 & s \\
\hline Reiten-Rubriken in SWS & Nicht geritten/keine Angabe & 22 & $5,2 \%$ & 14 & $11,1 \%$ & 1,97 & s \\
\hline
\end{tabular}




\begin{tabular}{|c|c|c|c|c|c|c|c|}
\hline & & $\begin{array}{c}\text { PP } \\
\text { Keine } \\
\text { vorz. } \\
\text { Wehen }\end{array}$ & & $\begin{array}{c}\text { PP mit } \\
\text { vorz. } \\
\text { Wehen }\end{array}$ & & Wurzel S & $\begin{array}{l}\text { Signi- } \\
\text { fikanz }\end{array}$ \\
\hline & $n=$ & 423 & & 126 & & & \\
\hline Reit-Belastung insgesamt & Leichte bis mäßige Bel & 274 & $64,8 \%$ & 79 & $62,7 \%$ & 0,42 & ns \\
\hline Reit-Belastung insgesamt & Leistungs- u. Spitzensport & 127 & $30,0 \%$ & 33 & $26,2 \%$ & 0,85 & ns \\
\hline Unfallrisiko & $\begin{array}{l}\text { fühlte mich vollig sicher, } \\
\text { Unfall ausgeschlossen }\end{array}$ & 202 & $47,8 \%$ & 45 & $35,7 \%$ & 2,45 & s \\
\hline $\begin{array}{l}\text { Unfall beim Reiten oder beim } \\
\text { Umgang mit dem Pferd }\end{array}$ & $\begin{array}{l}\text { ja (10 Frauen mit } \\
\text { Mehrfachnennungen) }\end{array}$ & 43 & $10,2 \%$ & 9 & $7,1 \%$ & 1,11 & ns \\
\hline $\begin{array}{l}\text { Unfallfolgen (\% bezogen auf } \\
\text { Unfälle) }\end{array}$ & $\begin{array}{l}\text { Unfall blieb ohne Folgen für } \\
\text { die Schwangerschaft }\end{array}$ & 43 & $100,0 \%$ & 7 & $77,8 \%$ & 6,00 & s \\
\hline Vorzeitiger BS & insgesamt ja & 25 & $5,9 \%$ & 39 & $31,0 \%$ & 5,86 & s \\
\hline Zervixinsuffizienz & bis 1 Woche danach & 14 & $3,3 \%$ & 11 & $8,7 \%$ & 2,04 & s \\
\hline Zervixinsuffizienz & später & 11 & $2,6 \%$ & 28 & $22,2 \%$ & 5,19 & $\mathrm{~s}$ \\
\hline Zervixinsuffizienz & insgesamt nein & 398 & $94,1 \%$ & 87 & $69,0 \%$ & 5,86 & $\mathrm{~s}$ \\
\hline Krankenhausaufenthalt & Komplikationen & 103 & $24,3 \%$ & 50 & $39,7 \%$ & 3,17 & $\mathrm{~s}$ \\
\hline \multirow[t]{2}{*}{ Krankenhausaufenthalt } & 29.-32. Woche & 13 & $3,1 \%$ & 18 & $14,3 \%$ & 3,47 & $\mathrm{~s}$ \\
\hline & nach 33. Woche & 60 & $14,2 \%$ & 61 & $48,4 \%$ & 7,18 & s \\
\hline Krankenhausaufenthalts & 8-14 Tage & 22 & $5,2 \%$ & 15 & $11,9 \%$ & 2,18 & $\mathrm{~s}$ \\
\hline \multirow[t]{2}{*}{ Bettruhe } & ja & 74 & $17,5 \%$ & 70 & $55,6 \%$ & 7,93 & s \\
\hline & nein & 331 & $78,3 \%$ & 53 & $42,1 \%$ & 7,49 & $\mathrm{~s}$ \\
\hline Antimykotika & ja & 36 & $8,5 \%$ & 22 & $17,5 \%$ & 2,46 & s \\
\hline \multirow[t]{2}{*}{ Wehenhemmung } & ja & 9 & $2,1 \%$ & 54 & $42,9 \%$ & 9,12 & $\mathrm{~s}$ \\
\hline & nein & 395 & $93,4 \%$ & 69 & $54,8 \%$ & 8,40 & $\mathrm{~s}$ \\
\hline Magnesium & ja & 72 & $17,0 \%$ & 86 & $68,3 \%$ & 11,31 & $\mathrm{~s}$ \\
\hline Magnesium & nein & 334 & $79,0 \%$ & 37 & $29,4 \%$ & 10,98 & $\mathrm{~s}$ \\
\hline Arbeitsbedingungen & belastet. & 5 & $1,2 \%$ & 7 & $5,6 \%$ & 2,08 & s \\
\hline Körperlich schwere Arbeit & kam vor & 143 & $33,8 \%$ & 44 & $34,9 \%$ & 0,23 & ns \\
\hline Körperlich schwere Arbeit & belastet. & 16 & $3,8 \%$ & 13 & $10,3 \%$ & 2,28 & s \\
\hline \multirow[t]{2}{*}{ Durch Familie, Beruf oder anderes } & $\begin{array}{l}\text { Sehr häufig und häufig } \\
\text { belastet }\end{array}$ & 101 & $23,9 \%$ & 47 & $37,3 \%$ & 2,81 & s \\
\hline & Sehr stark und stark belastet & 99 & $23,4 \%$ & 42 & $33,3 \%$ & 2,12 & s \\
\hline \multirow[t]{2}{*}{ Ende in Sws-Woche - Mittelwert } & nach 40. Woche & 142 & $33,6 \%$ & 27 & $21,4 \%$ & 2,81 & $\mathrm{~s}$ \\
\hline & gesamt ab 37 . Woche & 392 & $92,7 \%$ & 109 & $86,5 \%$ & 1,87 & ns \\
\hline Ende in Sws-Woche - Mittelwert & SSW) & 54 & $12,8 \%$ & 8 & $6,3 \%$ & 2,37 & $\mathrm{~s}$ \\
\hline \multirow[t]{2}{*}{ Ende in Sws-Woche - Mittelwert } & 36. Woche & 31 & $7,3 \%$ & 17 & $13,5 \%$ & 1,87 & ns \\
\hline & spontan & 239 & $56,5 \%$ & 77 & $61,1 \%$ & 0,93 & ns \\
\hline \multirow[t]{3}{*}{ Entbindung } & geplanter Kaiserschnitt & 59 & $13,9 \%$ & 8 & $6,3 \%$ & 2,76 & $\mathrm{~s}$ \\
\hline & ungeplanter Kaiserschnitt & 80 & $18,9 \%$ & 29 & $23,0 \%$ & 0,98 & ns \\
\hline & Kaiserschnitt insgesamt & 139 & $32,9 \%$ & 37 & $29,4 \%$ & 0,75 & ns \\
\hline
\end{tabular}




\begin{tabular}{|c|c|c|c|c|c|c|c|}
\hline & & Absolut & & $\begin{array}{l}\text { Absolut } \\
\text { PP BEL }\end{array}$ & & & \\
\hline & $n=$ & 742 & & 58 & & & \\
\hline & & & & & & $\begin{array}{c}\text { Wurzel } \\
\mathrm{S}\end{array}$ & $\begin{array}{l}\text { Sig- } \\
\text { nifi- } \\
\text { kanz }\end{array}$ \\
\hline \multirow[t]{3}{*}{ Tägliche Reitdauer vor der SWS } & $1 \mathrm{~h}$ & 477 & $64,3 \%$ & 44 & $75,9 \%$ & 1,97 & $s$ \\
\hline & $2-3 \mathrm{~h}$ & 234 & $31,5 \%$ & 14 & $24,1 \%$ & 1,26 & ns \\
\hline & $4 \mathrm{~h}$ und mehr & 31 & $4,2 \%$ & 0 & $0,0 \%$ & 5,70 & ns \\
\hline \multirow[t]{5}{*}{ Dressur vor der Schwangerschaft } & insgesamt geritten & 629 & $84,8 \%$ & 56 & $96,6 \%$ & 4,34 & $\mathrm{~s}$ \\
\hline & leichte Belastung & 81 & $10,9 \%$ & 8 & $13,8 \%$ & 0,62 & ns \\
\hline & mittlere Belastung & 202 & $27,2 \%$ & 15 & $25,9 \%$ & 0,22 & ns \\
\hline & leistungsorientiertes Reiten & 294 & $39,6 \%$ & 31 & $53,4 \%$ & 2,03 & $\mathrm{~s}$ \\
\hline & Spitzensport & 52 & $7,0 \%$ & 2 & $3,4 \%$ & 1,41 & ns \\
\hline \multirow[t]{2}{*}{ Weitergeritten -ja/nein } & $\begin{array}{l}\text { Nach Feststellung der Sws } \\
\text { weitergeritten }\end{array}$ & & & & & & ns \\
\hline & $\mathrm{Ja}$ & 686 & $92,5 \%$ & 53 & $91,4 \%$ & 0,29 & ns \\
\hline Bettruhe & nein & 257 & $34,6 \%$ & 34 & $58,6 \%$ & 3,58 & $s$ \\
\hline Bettruhe & $\begin{array}{l}\text { keine Eingabe, da keine Therapie } \\
\text { in der SWS }\end{array}$ & 386 & $52,0 \%$ & 16 & $27,6 \%$ & 3,97 & s \\
\hline Antimykotika & nein & 324 & $43,7 \%$ & 38 & $65,5 \%$ & 3,35 & s \\
\hline Antibiotika & $\begin{array}{l}\text { keine Eingabe, da keine Therapie } \\
\text { in der SWS }\end{array}$ & 385 & $51,9 \%$ & 16 & $27,6 \%$ & & \\
\hline $\begin{array}{l}\text { Antibiotika /Antimykotika in den } 12 \\
\text { Monaten vor der Sws }\end{array}$ & nein & 618 & $83,3 \%$ & 54 & $93,1 \%$ & 2,72 & $\mathrm{~s}$ \\
\hline \multirow[t]{2}{*}{ Körperlich schwere Arbeit } & kam nicht vor & 471 & $63,5 \%$ & 44 & $75,9 \%$ & 2,11 & $\mathrm{~s}$ \\
\hline & kam vor & 271 & $36,5 \%$ & 14 & $24,1 \%$ & 2,11 & s \\
\hline $\begin{array}{l}\text { Ich fühlte mich durch Arbeit, Beruf, } \\
\text { Familie oder anderes in der } \\
\text { Schwangerschaft belastet: }\end{array}$ & Sehr häufig und häufig belastet & 169 & $22,8 \%$ & 10 & $17,2 \%$ & 1,08 & ns \\
\hline $\begin{array}{l}\text { Ich fühlte mich durch Arbeit, Beruf, } \\
\text { Familie oder anderes in der } \\
\text { Schwangerschaft belastet: }\end{array}$ & Sehr stark und stark belastet & 168 & $22,6 \%$ & 7 & $12,1 \%$ & 2,31 & s \\
\hline Nikotin & $\begin{array}{l}\text { vor der Sws schon länger nicht } \\
\text { geraucht }\end{array}$ & 102 & $13,7 \%$ & 16 & $27,6 \%$ & 2,32 & s \\
\hline Wein, Sekt, Obstwein & habe fast nie Wein etc. getrunken & 671 & $90,4 \%$ & 56 & $96,6 \%$ & 2,37 & s \\
\hline \multirow[t]{7}{*}{ Dauer der Schwangerschaft } & Ende in Sws-Woche - Mittelwert & 39,7 & & 38,6 & & & \\
\hline & 25.-26. Woche & 1 & $0,1 \%$ & 0 & $0,0 \%$ & 0,86 & ns \\
\hline & 27.-31. Woche & 1 & $0,1 \%$ & 1 & $1,7 \%$ & 0,94 & ns \\
\hline & 32.-36. Woche & 42 & $5,7 \%$ & 6 & $10,3 \%$ & 1,13 & ns \\
\hline & 37.-40. Woche & 435 & $58,6 \%$ & 40 & $69,0 \%$ & 1,64 & ns \\
\hline & nach 40. Woche & 263 & $35,4 \%$ & 11 & $19,0 \%$ & 3,01 & $\mathrm{~s}$ \\
\hline & gesamt ab 37. Woche & 698 & $94,1 \%$ & 51 & $87,9 \%$ & 1,42 & ns \\
\hline Dauer der Schwangerschaft & Gesamt nach 40+6 (41. SSW) & 102 & $13,7 \%$ & 2 & $3,4 \%$ & 3,82 & ns \\
\hline Dauer der Schwangerschaft & $\begin{array}{l}\text { Frühgeburt bis einschließlich } 36 . \\
\text { Woche }\end{array}$ & 44 & $5,9 \%$ & 7 & $12,1 \%$ & 1,42 & ns \\
\hline \multirow[t]{5}{*}{ Entbindung } & spontan & 509 & $68,6 \%$ & 8 & $13,8 \%$ & 11,33 & $\mathrm{~s}$ \\
\hline & geplanter Kaiserschnitt & 33 & $4,4 \%$ & 33 & $56,9 \%$ & 8,02 & $\mathrm{~s}$ \\
\hline & ungeplanter Kaiserschnitt & 119 & $16,0 \%$ & 15 & $25,9 \%$ & 1,68 & ns \\
\hline & Kaiserschnitt insgesamt & 152 & $20,5 \%$ & 48 & $82,8 \%$ & 12,05 & $\mathrm{~s}$ \\
\hline & Saugglocke oder Zange & 81 & $10,9 \%$ & 2 & $3,4 \%$ & 2,84 & ns \\
\hline \multirow[t]{4}{*}{ Verlauf der Entbindung } & ohne Schwierigkeiten & 417 & $56,2 \%$ & 44 & $75,9 \%$ & 3,34 & $\mathrm{~s}$ \\
\hline & mit Schwierigkeiten & 254 & $34,2 \%$ & 11 & $19,0 \%$ & 2,80 & $\mathrm{~s}$ \\
\hline & $\begin{array}{l}\text { Verlängerter Geburtsverlaut oder } \\
\text { Geb.-stillstand }\end{array}$ & 149 & $20,1 \%$ & 6 & $10,3 \%$ & 2,30 & s \\
\hline & $\begin{array}{l}\text { Freitext-Einträge zu schwierigen } \\
\text { Verläufen }\end{array}$ & 261 & $35,2 \%$ & 11 & $19,0 \%$ & 2,98 & $\mathrm{~s}$ \\
\hline
\end{tabular}


Reiten in der Schwangerschaft

Geritten bis zur Entbindung in Terminnähe - BEL vs. SL

\begin{tabular}{|c|c|c|c|c|c|c|}
\hline & $\begin{array}{c}\text { Absolut ET } \\
\text { ab SSW } 37 \\
\text { SL }\end{array}$ & & $\begin{array}{l}\text { AbsolutET ab } \\
\text { SS" 37, } \\
\text { BEL/QL }\end{array}$ & & & \\
\hline \multirow[t]{2}{*}{$n=$} & 169 & & 5 & & & \\
\hline & & & & & Wurzel S & Signi-fikanz \\
\hline frühere Geburten & & & & & & ns \\
\hline keine & 114 & $67,5 \%$ & 4 & $80,0 \%$ & & ns \\
\hline Frühere Kompl.: & & & & & & ns \\
\hline Kaiserschnitt & 11 & $6,5 \%$ & 1 & $20,0 \%$ & 0,75 & ns \\
\hline \multicolumn{7}{|l|}{ Sportarten vor der Schwangerschaft } \\
\hline keine Sportarten zusätzlich zum Reiten & 58 & $34,3 \%$ & 0 & $0,0 \%$ & 9,40 & ns \\
\hline Insgesamt sportl. aktiv zusätzlich zum Reiten & 111 & $65,7 \%$ & 5 & $100,0 \%$ & 9,40 & $\mathrm{~s}$ \\
\hline Ausdauerbetonte Sportarten & 82 & $48,5 \%$ & 5 & $100,0 \%$ & 13,39 & $\mathrm{~s}$ \\
\hline Kraftbetonte Sportarten & 17 & $10,1 \%$ & 0 & $0,0 \%$ & 4,35 & ns \\
\hline Gymnastische Sportarten & 17 & $10,1 \%$ & 0 & $0,0 \%$ & 4,35 & ns \\
\hline Fun- und Risikosportarten & 24 & $14,2 \%$ & 0 & $0,0 \%$ & 5,29 & ns \\
\hline Spielsportarten & 12 & $7,1 \%$ & 0 & $0,0 \%$ & 3,59 & ns \\
\hline \multicolumn{7}{|l|}{ Tägliche Reitdauer vor der SWS } \\
\hline $1 \mathrm{~h}$ & 100 & $59,2 \%$ & 5 & $100,0 \%$ & 10,80 & $\mathrm{~s}$ \\
\hline $2-3 \mathrm{~h}$ & 54 & $32,0 \%$ & 0 & $0,0 \%$ & 8,91 & ns \\
\hline $4 \mathrm{~h}$ und mehr & 15 & $8,9 \%$ & 0 & $0,0 \%$ & 4,06 & ns \\
\hline \multicolumn{7}{|l|}{ Dressur } \\
\hline insgesamt geritten & 142 & $84,0 \%$ & 5 & $100,0 \%$ & 5,67 & $\mathrm{~s}$ \\
\hline leichte Belastung & 21 & $12,4 \%$ & 0 & $0,0 \%$ & 4,90 & ns \\
\hline mittlere Belastung & 37 & $21,9 \%$ & 2 & $40,0 \%$ & 0,82 & ns \\
\hline leistungsorientiertes Reiten & 62 & $36,7 \%$ & 2 & $40,0 \%$ & 0,15 & ns \\
\hline Spitzensport & 22 & $13,0 \%$ & 1 & $20,0 \%$ & 0,39 & ns \\
\hline \multicolumn{7}{|l|}{ Nach Feststellung der Sws weitergeritten } \\
\hline $\mathrm{Ja}$ & 168 & $99,4 \%$ & 5 & $100,0 \%$ & 1,00 & \\
\hline und zwar bis zur Schwangerschaftswoche & 39,43 & & 38,80 & & & \\
\hline \multicolumn{7}{|c|}{\begin{tabular}{|l|l} 
St-Abweichung &
\end{tabular}} \\
\hline \multicolumn{2}{|c|}{ Reiten in h/Woche während der Schwangerschaft } & & & & \#DIV/0! & \\
\hline bis $2 \mathrm{~h}$ & 38 & $22,5 \%$ & 0 & $0,0 \%$ & 7,00 & ns \\
\hline $3-5 \mathrm{~h}$ & 80 & $47,3 \%$ & 5 & $100,0 \%$ & 13,71 & $\mathrm{~s}$ \\
\hline 7h und mehr & 51 & $30,2 \%$ & 0 & $0,0 \%$ & & ns \\
\hline \multicolumn{7}{|l|}{ Reiterliche Disziplinen während der Sws } \\
\hline \multicolumn{7}{|l|}{ Dressur } \\
\hline insgesamt geritten & 125 & $74,0 \%$ & 5 & $100,0 \%$ & 7,71 & $\mathrm{~s}$ \\
\hline leichte Belastung & 31 & $18,3 \%$ & 0 & $0,0 \%$ & 6,16 & ns \\
\hline mittlere Belastung & 44 & $26,0 \%$ & 5 & $100,0 \%$ & 21,91 & $\mathrm{~s}$ \\
\hline leistungsorientiertes Reiten & 37 & $21,9 \%$ & 0 & $0,0 \%$ & 6,88 & ns \\
\hline Spitzensport & 13 & $7,7 \%$ & 0 & $0,0 \%$ & 3,75 & ns \\
\hline Gesamt Dressur geritten & & & & & & ns \\
\hline \multicolumn{7}{|l|}{ ENTBINDUNG } \\
\hline \multicolumn{7}{|l|}{ Dauer der Schwangerschaft } \\
\hline 25.-26. Woche & 0 & $0,0 \%$ & 0 & $0,0 \%$ & & \\
\hline 27.-31. Woche & 0 & $0,0 \%$ & 0 & $0,0 \%$ & & \\
\hline 32.-36. Woche & 1 & $0,6 \%$ & 0 & $0,0 \%$ & 1,00 & ns \\
\hline 37.-40. Woche & 103 & $60,9 \%$ & 3 & $60,0 \%$ & 0,04 & ns \\
\hline nach 40. Woche & 65 & $38,5 \%$ & 2 & $40,0 \%$ & 0,07 & ns \\
\hline gesamt ab 37. Woche & 168 & $99,4 \%$ & 5 & $100,0 \%$ & 1,00 & ns \\
\hline Gesamt nach 40+6 (41. SSW) & 25 & $14,8 \%$ & 0 & $0,0 \%$ & 5,42 & ns \\
\hline Frühgeburt bis einschließlich 36 . Woche & 1 & $0,6 \%$ & 0 & $0,0 \%$ & 1,00 & ns \\
\hline
\end{tabular}


Reiten in der Schwangerschaft

Anhang Tab. A6

Geritten bis zur Entbindung in Terminnähe - BEL vs. SL

\begin{tabular}{|c|c|c|c|c|c|c|}
\hline & $\begin{array}{c}\text { ET ab SSW } \\
37, \mathrm{SL}\end{array}$ & & $\begin{array}{l}\text { ET ab SSW } \\
37, \text { BEL/QL }\end{array}$ & & & \\
\hline$n=$ & 169 & & 5 & & & \\
\hline & & & & & Wurzel S & Signi-fikanz \\
\hline \multicolumn{7}{|l|}{ Gewicht und Größe des Kindes } \\
\hline \multicolumn{7}{|l|}{ Körpergewicht in g } \\
\hline Mittelwert & 3440,2367 & & 2991 & & & \\
\hline bis $600 \mathrm{~g}$ & 0 & $0,0 \%$ & 0 & $0,0 \%$ & & \\
\hline $601-1000 \mathrm{~g}$ & 0 & $0,0 \%$ & 0 & $0,0 \%$ & & \\
\hline $1001-1500 \mathrm{~g}$ & 0 & $0,0 \%$ & 0 & $0,0 \%$ & & \\
\hline $1501-2000 \mathrm{~g}$ & 0 & $0,0 \%$ & 0 & $0,0 \%$ & & \\
\hline $2001-2500 \mathrm{~g}$ & 4 & $2,4 \%$ & 0 & $0,0 \%$ & 2,02 & ns \\
\hline $2501-3000 \mathrm{~g}$ & 20 & $11,8 \%$ & 3 & $60,0 \%$ & 2,18 & ns \\
\hline $3001-3500 \mathrm{~g}$ & 79 & $46,7 \%$ & 2 & $40,0 \%$ & 0,30 & ns \\
\hline $3501-4000 \mathrm{~g}$ & 51 & $30,2 \%$ & 0 & $0,0 \%$ & 8,55 & ns \\
\hline $4001-4500 \mathrm{~g}$ & 14 & $8,3 \%$ & 0 & $0,0 \%$ & 3,91 & ns \\
\hline über $4500 \mathrm{~g}$ & 1 & $0,6 \%$ & 0 & $0,0 \%$ & 1,00 & ns \\
\hline \multicolumn{7}{|l|}{ Körpergröße in cm } \\
\hline Mittelwert & 51,668639 & & 49,4 & & & \\
\hline bis $30 \mathrm{~cm}$ & 0 & $0,0 \%$ & 0 & $0,0 \%$ & & \\
\hline $31-40 \mathrm{~cm}$ & 0 & $0,0 \%$ & 0 & $0,0 \%$ & & \\
\hline $41-50 \mathrm{~cm}$ & 52 & $30,8 \%$ & 4 & $80,0 \%$ & 2,70 & ns \\
\hline über $50 \mathrm{~cm}$ & 116 & $68,6 \%$ & 1 & $20,0 \%$ & 2,67 & ns \\
\hline \multicolumn{7}{|l|}{ Entbindung } \\
\hline spontan & 126 & $74,6 \%$ & 2 & $40,0 \%$ & 1,56 & ns \\
\hline geplanter Kaiserschnitt & 7 & $4,1 \%$ & 1 & $20,0 \%$ & 0,88 & ns \\
\hline ungeplanter Kaiserschnitt & 20 & $11,8 \%$ & 1 & $20,0 \%$ & 0,45 & ns \\
\hline Kaiserschnitt insgesamt & 27 & $16,0 \%$ & 2 & $40,0 \%$ & 1,09 & ns \\
\hline Saugglocke oder Zange & 16 & $9,5 \%$ & 1 & $20,0 \%$ & 0,58 & ns \\
\hline \multicolumn{7}{|l|}{ Verlauf der Entbindung } \\
\hline ohne Schwierigkeiten & 112 & $66,3 \%$ & 4 & $80,0 \%$ & 0,75 & ns \\
\hline mit Schwierigkeiten & 48 & $28,4 \%$ & 0 & $0,0 \%$ & 8,19 & ns \\
\hline Verlängerter Geburtsverlauf oder Geb.-stillstand & 19 & $11,2 \%$ & 2 & $40,0 \%$ & 1,30 & ns \\
\hline Freitext-Einträge zu schwierigen Verläufen & 49 & $29,0 \%$ & 0 & $0,0 \%$ & 8,31 & ns \\
\hline Kindslage - Angaben insgesamt & 169 & $100,0 \%$ & 5 & $100,0 \%$ & & \\
\hline unbekannt & 0 & $0,0 \%$ & 0 & $0,0 \%$ & & \\
\hline Normale Schädellage (\% aller bekannten Kindsl & 163 & $96,4 \%$ & 0 & $0,0 \%$ & 67,76 & ns \\
\hline Komplizierte Schädellage (\% aller bekannten Kil & 6 & $3,6 \%$ & 0 & $0,0 \%$ & 2,49 & ns \\
\hline Beckenendlage (\% aller bekannten Kindslagen) & 0 & $0,0 \%$ & 4 & $80,0 \%$ & 4,47 & ns \\
\hline Quer- oder Schräglage (\% aller bekannten Kind: & 0 & $0,0 \%$ & 1 & $20,0 \%$ & 1,12 & ns \\
\hline \multicolumn{7}{|l|}{ Kind nach der Geburt } \\
\hline gesund geboren & 163 & $96,4 \%$ & 5 & $100,0 \%$ & 2,49 & $\mathrm{~s}$ \\
\hline krank geboren & 6 & $3,6 \%$ & 0 & $0,0 \%$ & 2,49 & ns \\
\hline Totgeburt & 0 & $0,0 \%$ & 0 & $0,0 \%$ & & \\
\hline Freitext-Einträge & 6 & $3,6 \%$ & 0 & $0,0 \%$ & 2,49 & ns \\
\hline \multicolumn{7}{|l|}{ Kind (er) nach einem Jahr } \\
\hline Kind (alle Kinder) gesund & 167 & $98,8 \%$ & 5 & $100,0 \%$ & 1,42 & ns \\
\hline Kind (bzw. eines der Kinder) krank & 1 & $0,6 \%$ & 0 & $0,0 \%$ & 1,00 & ns \\
\hline Kind (bzw. eines der Kinder) verstorben & 0 & $0,0 \%$ & 0 & $0,0 \%$ & & \\
\hline Freitext-Einträge & 1 & $0,6 \%$ & 0 & $0,0 \%$ & 1,00 & ns \\
\hline
\end{tabular}


Primiparae

Spontanentbindungen vs.

vaginal-operative Entbindungen

\begin{tabular}{|c|c|c|c|c|c|c|c|c|}
\hline & $\begin{array}{c}\text { Absolut } \\
\text { Primi- } \\
\text { parae }\end{array}$ & & $\begin{array}{l}\text { Absolut } \\
\text { spontan }\end{array}$ & & $\begin{array}{l}\text { Absolut } \\
\text { vag.-op. } \\
\text { Entbind. }\end{array}$ & & Wurzel S & $\begin{array}{l}\text { Signi- } \\
\text { fikanz }\end{array}$ \\
\hline$n=$ & 1122 & & 703 & & 126 & & & \\
\hline \multicolumn{9}{|l|}{ Bisherige Schwangerschaften } \\
\hline keine & 1009 & $89,9 \%$ & 631 & $89,8 \%$ & 121 & $96,0 \%$ & 3,01 & $\mathrm{~s}$ \\
\hline \multicolumn{9}{|l|}{$\begin{array}{l}\text { Sportlicher Trainingszustand vor der } \\
\text { Schwangerschaft }\end{array}$} \\
\hline ausgezeichnet (täglich mind. 1 h Sport) & 281 & $25,0 \%$ & 181 & $25,7 \%$ & 30 & $23,8 \%$ & 0,47 & ns \\
\hline sehr gut (4h und mehr Sport pro Woche) & 352 & $31,4 \%$ & 211 & $30,0 \%$ & 37 & $29,4 \%$ & 0,15 & ns \\
\hline gut (2-4h Sport pro Woche) & 348 & $31,0 \%$ & 217 & $30,9 \%$ & 40 & $31,7 \%$ & 0,20 & ns \\
\hline mäßig (1-2 h Sport pro Woche) & 107 & $9,5 \%$ & 70 & $10,0 \%$ & 17 & $13,5 \%$ & 1,09 & ns \\
\hline $\begin{array}{l}\text { nicht besonders (weniger als } 1 \mathrm{~h} \text { Sport } \\
\text { pro Wo.) }\end{array}$ & 34 & $3,0 \%$ & 24 & $3,4 \%$ & 2 & $1,6 \%$ & 1,40 & ns \\
\hline \multicolumn{9}{|l|}{ Leistungsstand Reiten } \\
\hline Ungeübte Reiterin & 24 & $2,1 \%$ & 14 & $2,0 \%$ & 6 & $4,8 \%$ & 1,41 & ns \\
\hline Geübte Reiterin & 737 & $65,7 \%$ & 461 & $65,6 \%$ & 76 & $60,3 \%$ & 1,12 & ns \\
\hline $\begin{array}{l}\text { Leistungssportlerin im gehobenen } \\
\text { Bereich }\end{array}$ & 315 & $28,1 \%$ & 202 & $28,7 \%$ & 39 & $31,0 \%$ & 0,50 & ns \\
\hline Berufsreiterin & 46 & $4,1 \%$ & 26 & $3,7 \%$ & 5 & $4,0 \%$ & 0,14 & ns \\
\hline \multicolumn{9}{|c|}{ Nach Feststellung der Sws weitergeritten } \\
\hline $\mathrm{Ja}$ & 1039 & $92,6 \%$ & 654 & $93,0 \%$ & 118 & $93,7 \%$ & 0,26 & ns \\
\hline $\begin{array}{l}\text { und zwar bis zur } \\
\text { Schwangerschaftswoche }\end{array}$ & 28,9 & & 28,8 & & 29,7 & & & ns \\
\hline \multicolumn{9}{|l|}{ Reitzeit pro Woche } \\
\hline bis $0,2 \mathrm{~h}$ & 24 & $2,1 \%$ & 17 & $2,4 \%$ & 0 & $0,0 \%$ & 4,17 & ns \\
\hline ca. $0,5 \mathrm{~h}$ & 6 & $0,5 \%$ & 3 & $0,4 \%$ & 0 & $0,0 \%$ & 1,74 & ns \\
\hline $1 \mathrm{~h}$ & 66 & $5,9 \%$ & 43 & $6,1 \%$ & 10 & $7,9 \%$ & 0,71 & ns \\
\hline $2 \mathrm{~h}$ & 247 & $22,0 \%$ & 159 & $22,6 \%$ & 23 & $18,3 \%$ & 1,15 & ns \\
\hline $4 \mathrm{~h}$ & 486 & $43,3 \%$ & 301 & $42,8 \%$ & 56 & $44,4 \%$ & 0,34 & ns \\
\hline $5 \mathrm{~h}$ & 34 & $3,0 \%$ & 25 & $3,6 \%$ & 4 & $3,2 \%$ & 0,22 & ns \\
\hline $7 \mathrm{~h}$ & 114 & $10,2 \%$ & 72 & $10,2 \%$ & 16 & $12,7 \%$ & 0,77 & ns \\
\hline $10 \mathrm{~h}$ & 68 & $6,1 \%$ & 41 & $5,8 \%$ & 8 & $6,3 \%$ & 0,22 & ns \\
\hline $17,5 \mathrm{~h}$ & 57 & $5,1 \%$ & 38 & $5,4 \%$ & 7 & $5,6 \%$ & 0,07 & ns \\
\hline $35 \mathrm{~h}$ & 9 & $0,8 \%$ & 4 & $0,6 \%$ & 1 & $0,8 \%$ & 0,27 & ns \\
\hline \multicolumn{9}{|l|}{ Welche Pferde geritten } \\
\hline überwiegend Schulpferde & 16 & $1,4 \%$ & 10 & $1,4 \%$ & 2 & $1,6 \%$ & 0,14 & ns \\
\hline eigenes Pferd / Beteiligung & 919 & $81,9 \%$ & 575 & $81,8 \%$ & 108 & $85,7 \%$ & 1,14 & ns \\
\hline $\begin{array}{l}\text { mehrere Privatpferde / } \\
\text { Ausbildungspferde }\end{array}$ & 109 & $9,7 \%$ & 73 & $10,4 \%$ & 8 & $6,3 \%$ & 1,64 & ns \\
\hline keine Angabe & 78 & $7,0 \%$ & 45 & $6,4 \%$ & 8 & $6,3 \%$ & 0,02 & ns \\
\hline \multicolumn{9}{|l|}{$\begin{array}{l}\text { Reiten in h/Woche während der } \\
\text { Schwangerschaft }\end{array}$} \\
\hline bis $2 \mathrm{~h}$ & & & 222 & $31,6 \%$ & 33 & $26,2 \%$ & 1,26 & ns \\
\hline $3-5 \mathrm{~h}$ & & & 326 & $46,4 \%$ & 60 & $47,6 \%$ & 0,26 & ns \\
\hline 7h und mehr & & & 155 & $22,0 \%$ & 32 & $25,4 \%$ & 0,80 & ns \\
\hline \multicolumn{9}{|l|}{ Reiten-Rubriken } \\
\hline Dressurbetont, leichte Belastung & 363 & $32,4 \%$ & 235 & $33,4 \%$ & 44 & $34,9 \%$ & 0,32 & ns \\
\hline Dressurbetont, ambitionierte Belastung & 218 & $19,4 \%$ & 133 & $18,9 \%$ & 24 & $19,0 \%$ & 0,03 & ns \\
\hline Springbetont, leichte Belastung & 19 & $1,7 \%$ & 11 & $1,6 \%$ & 2 & $1,6 \%$ & 0,02 & ns \\
\hline Springbetont, ambitionierte Belastung & 63 & $5,6 \%$ & 41 & $5,8 \%$ & 6 & $4,8 \%$ & 0,51 & ns \\
\hline
\end{tabular}


Primiparae

Spontanentbindungen vs.

vaginal-operative Entbindungen

\begin{tabular}{|c|c|c|c|c|c|c|c|c|}
\hline & $\begin{array}{c}\text { Absolut } \\
\text { Primi- } \\
\text { parae }\end{array}$ & & $\begin{array}{l}\text { Absolut } \\
\text { spontan }\end{array}$ & & $\begin{array}{l}\text { Absolut } \\
\text { vag.-op. } \\
\text { Entbind. }\end{array}$ & & Wurzel S & $\begin{array}{l}\text { Signi- } \\
\text { fikanz }\end{array}$ \\
\hline$n=$ & 1122 & & 703 & & 126 & & & \\
\hline Nicht geritten/keine Angabe & 68 & $6,1 \%$ & 39 & $5,5 \%$ & 7 & $5,6 \%$ & 0,00 & ns \\
\hline \multicolumn{9}{|l|}{$\begin{array}{l}\text { Trainingszustand während der } \\
\text { Schwangerschaft }\end{array}$} \\
\hline ausgezeichnet & 138 & $12,3 \%$ & 89 & $12,7 \%$ & 14 & $11,1 \%$ & 0,50 & ns \\
\hline sehr gut & 220 & $19,6 \%$ & 132 & $18,8 \%$ & 27 & $21,4 \%$ & 0,67 & ns \\
\hline gut & 391 & $34,8 \%$ & 244 & $34,7 \%$ & 45 & $35,7 \%$ & 0,22 & ns \\
\hline mäßig & 260 & $23,2 \%$ & 163 & $23,2 \%$ & 34 & $27,0 \%$ & 0,89 & ns \\
\hline nicht besonders & 113 & $10,1 \%$ & 75 & $10,7 \%$ & 6 & $4,8 \%$ & 2,65 & $\mathrm{~s}$ \\
\hline \multicolumn{9}{|l|}{ ENTBINDUNG } \\
\hline \multicolumn{9}{|l|}{ Dauer der Schwangerschaft } \\
\hline Ende in Sws-Woche - Mittelwert & 39,579323 & & 39,6 & & 39,968254 & & & \\
\hline 25.-26. Woche & 2 & $0,2 \%$ & 1 & $0,1 \%$ & 0 & $0,0 \%$ & 1,00 & ns \\
\hline 27.-31. Woche & 4 & $0,4 \%$ & 2 & $0,3 \%$ & 1 & $0,8 \%$ & 0,62 & ns \\
\hline 32.-36. Woche & 64 & $5,7 \%$ & 37 & $5,3 \%$ & 3 & $2,4 \%$ & 1,80 & ns \\
\hline 37.-40. Woche & 679 & $60,5 \%$ & 437 & $62,2 \%$ & 72 & $57,1 \%$ & 1,05 & ns \\
\hline nach 40. Woche & 373 & $33,2 \%$ & 226 & $32,1 \%$ & 50 & $39,7 \%$ & 1,60 & ns \\
\hline gesamt ab 37 . Woche & 1052 & $93,8 \%$ & 663 & $94,3 \%$ & 122 & $96,8 \%$ & 1,41 & ns \\
\hline Gesamt nach 40+6 (41. SSW) & & & 76 & $10,8 \%$ & 21 & $16,7 \%$ & 1,66 & ns \\
\hline Frühgeburt bis einschließlich 36 . Woche & 70 & $6,2 \%$ & 40 & $5,7 \%$ & 4 & $3,2 \%$ & 1,41 & ns \\
\hline \multicolumn{9}{|l|}{ Gewicht und Größe des Kindes } \\
\hline \multicolumn{9}{|l|}{ Körpergewicht in g } \\
\hline Mittelwert & 3389 & & 3379 & & 3475 & & & ns \\
\hline bis $600 \mathrm{~g}$ & 1 & $0,1 \%$ & 0 & $0,0 \%$ & 0 & $0,0 \%$ & & \\
\hline $601-1000 \mathrm{~g}$ & 1 & $0,1 \%$ & 0 & $0,0 \%$ & 0 & $0,0 \%$ & & \\
\hline $1001-1500 \mathrm{~g}$ & 2 & $0,2 \%$ & 1 & $0,1 \%$ & 1 & $0,8 \%$ & 0,81 & ns \\
\hline $1501-2000 \mathrm{~g}$ & 6 & $0,5 \%$ & 0 & $0,0 \%$ & 2 & $1,6 \%$ & 1,43 & ns \\
\hline $2001-2500 \mathrm{~g}$ & 44 & $3,9 \%$ & 24 & $3,4 \%$ & 0 & $0,0 \%$ & 4,98 & ns \\
\hline $2501-3000 \mathrm{~g}$ & 179 & $16,0 \%$ & 121 & $17,2 \%$ & 11 & $8,7 \%$ & 2,94 & ns \\
\hline $3001-3500 \mathrm{~g}$ & 451 & $40,2 \%$ & 300 & $42,7 \%$ & 54 & $42,9 \%$ & 0,04 & ns \\
\hline $3501-4000 \mathrm{~g}$ & 325 & $29,0 \%$ & 199 & $28,3 \%$ & 41 & $32,5 \%$ & 0,94 & ns \\
\hline $4001-4500 \mathrm{~g}$ & 95 & $8,5 \%$ & 50 & $7,1 \%$ & 13 & $10,3 \%$ & 1,11 & ns \\
\hline über $4500 \mathrm{~g}$ & 18 & $1,6 \%$ & 8 & $1,1 \%$ & 4 & $3,2 \%$ & 1,26 & ns \\
\hline \multicolumn{9}{|l|}{ Körpergröße in cm } \\
\hline Mittelwert & 51,4 & & 51,4 & & 52,1 & & & \\
\hline bis $30 \mathrm{~cm}$ & 3 & $0,3 \%$ & 1 & $0,1 \%$ & 0 & $0,0 \%$ & 1,00 & ns \\
\hline $31-40 \mathrm{~cm}$ & 7 & $0,6 \%$ & 5 & $0,7 \%$ & 0 & $0,0 \%$ & 2,24 & ns \\
\hline $41-50 \mathrm{~cm}$ & 374 & $33,3 \%$ & 234 & $33,3 \%$ & 38 & $30,2 \%$ & 0,70 & ns \\
\hline über $50 \mathrm{~cm}$ & 736 & $65,6 \%$ & 461 & $65,6 \%$ & 88 & $69,8 \%$ & 0,96 & ns \\
\hline \multicolumn{9}{|l|}{ Verlauf der Entbindung } \\
\hline ohne Schwierigkeiten & 647 & $57,7 \%$ & 503 & $71,6 \%$ & 22 & $17,5 \%$ & 14,29 & ns \\
\hline mit Schwierigkeiten & 368 & $32,8 \%$ & 155 & $22,0 \%$ & 74 & $58,7 \%$ & 7,88 & ns \\
\hline $\begin{array}{l}\text { Verlängerter Geburtsverlauf oder Geb.- } \\
\text { stillstand }\end{array}$ & 214 & $19,1 \%$ & 83 & $11,8 \%$ & 60 & $47,6 \%$ & 7,76 & ns \\
\hline $\begin{array}{l}\text { Freitext-Einträge zu schwierigen } \\
\text { Verläufen }\end{array}$ & 386 & $34,4 \%$ & 167 & $23,8 \%$ & 76 & $60,3 \%$ & 7,87 & ns \\
\hline Kindslage - Angaben insgesamt & 800 & $71,3 \%$ & 517 & $73,5 \%$ & 83 & $65,9 \%$ & 1,69 & ns \\
\hline unbekannt & 322 & $28,7 \%$ & 186 & $26,5 \%$ & 43 & $34,1 \%$ & 1,69 & ns \\
\hline $\begin{array}{l}\text { Normale Schädellage (\% aller } \\
\text { bekannten Kindslagen) }\end{array}$ & 699 & $87,4 \%$ & 500 & $96,7 \%$ & 71 & $85,5 \%$ & 3,49 & ns \\
\hline
\end{tabular}


Reiten in der Schwangerschaft

Primiparae

Spontanentbindungen vs.

vaginal-operative Entbindungen

\begin{tabular}{|c|c|c|c|c|c|c|c|c|}
\hline & $\begin{array}{c}\text { Absolut } \\
\text { Primi- } \\
\text { parae }\end{array}$ & & $\begin{array}{l}\text { Absolut } \\
\text { spontan }\end{array}$ & & $\begin{array}{l}\text { Absolut } \\
\text { vag.-op. } \\
\text { Entbind. }\end{array}$ & & Wurzel S & $\begin{array}{l}\text { Signi- } \\
\text { fikanz }\end{array}$ \\
\hline$n=$ & 1122 & & 703 & & 126 & & & \\
\hline $\begin{array}{l}\text { Komplizierte Schädellage (\% aller } \\
\text { bekannten Kindslagen) }\end{array}$ & 43 & $5,4 \%$ & 9 & $1,7 \%$ & 10 & $12,0 \%$ & 3,50 & ns \\
\hline $\begin{array}{l}\text { Beckenendlage (\% aller bekannten } \\
\text { Kindslagen) }\end{array}$ & 51 & $6,4 \%$ & 7 & $1,4 \%$ & 2 & $2,4 \%$ & 0,74 & ns \\
\hline $\begin{array}{l}\text { Quer- oder Schräglage (\% aller } \\
\text { bekannten Kindslagen) }\end{array}$ & 7 & $0,9 \%$ & 1 & $0,2 \%$ & 0 & $0,0 \%$ & 1,17 & ns \\
\hline \multicolumn{9}{|l|}{ Kind nach der Geburt } \\
\hline gesund geboren & 1061 & $94,6 \%$ & 669 & $95,2 \%$ & 119 & $94,4 \%$ & 0,33 & ns \\
\hline krank geboren & 61 & $5,4 \%$ & 34 & $4,8 \%$ & 7 & $5,6 \%$ & 0,33 & ns \\
\hline Totgeburt & 0 & $0,0 \%$ & 0 & $0,0 \%$ & 0 & $0,0 \%$ & & \\
\hline
\end{tabular}


Reiten in der Schwangerschaft

Primiparae

Spontanentbindungen vs. sekundäre Sectiones

\begin{tabular}{|c|c|c|c|c|c|c|}
\hline & $\begin{array}{l}\text { Absolut PP } \\
\text { Spontan }\end{array}$ & & $\begin{array}{l}\text { Absolut PP } \\
\text { Sekundäre } \\
\text { Sectio }\end{array}$ & & Wurzel S & $\begin{array}{l}\text { Signi- } \\
\text { fikanz }\end{array}$ \\
\hline $\mathrm{n}=$ & 703 & & 186 & & & \\
\hline \multicolumn{7}{|l|}{ Alter zu Beginn des SS } \\
\hline$<18$ Jahre & 7 & $1,0 \%$ & 0 & $0,0 \%$ & 2,66 & ns \\
\hline 18 bis 24 Jahre & 123 & $17,5 \%$ & 16 & $8,6 \%$ & 3,55 & $s$ \\
\hline 25-29 Jahre & 283 & $40,3 \%$ & 68 & $36,6 \%$ & 0,93 & ns \\
\hline 30-34 Jahre & 236 & $33,6 \%$ & 76 & $40,9 \%$ & 1,81 & ns \\
\hline 35-39 Jahre & 49 & $7,0 \%$ & 24 & $12,9 \%$ & 2,25 & $\mathrm{~s}$ \\
\hline 40 Jahre und mehr & 5 & $0,7 \%$ & 2 & $1,1 \%$ & 0,44 & ns \\
\hline Gesamt & 703 & $100,0 \%$ & 186 & $100,0 \%$ & & ns \\
\hline Mittelwert & 28,5 & & 30,1 & & 0,35 & $\mathrm{~s}$ \\
\hline s & 4,4 & & 4,3 & & & \\
\hline \multicolumn{7}{|l|}{ BMI } \\
\hline Untergewicht <20 & 135 & $19,2 \%$ & 35 & $18,8 \%$ & 0,12 & ns \\
\hline Normalgewicht 20-24,9 & 471 & $67,0 \%$ & 112 & $60,2 \%$ & 1,69 & ns \\
\hline Übergewicht > 25 & 97 & $13,8 \%$ & 39 & $20,6 \%$ & 2,10 & $s$ \\
\hline keine Angabe & 0 & $0,0 \%$ & 0 & $0,0 \%$ & & \\
\hline Summe & 703 & $100,0 \%$ & 186 & $100,0 \%$ & & ns \\
\hline BMI Mittelwert & 22,2 & & 22,8 & & & \\
\hline Standardabweichung & 2,9 & & 3,5 & & & $\mathrm{~s}$ \\
\hline \multicolumn{7}{|l|}{ frühere Gravidität } \\
\hline keine & 631 & $89,8 \%$ & 164 & $88,2 \%$ & 0,60 & ns \\
\hline \multicolumn{7}{|l|}{ Frühere Kompl.: } \\
\hline Summe der Nennungen & 90 & $12,8 \%$ & 41 & $22,0 \%$ & 2,81 & s \\
\hline $\begin{array}{l}\text { Sportlicher Trainingszustand vor der } \\
\text { Schwangerschaft }\end{array}$ & & & & & & ns \\
\hline ausgezeichnet (täglich mind. 1 h Sport) & 181 & $25,7 \%$ & 37 & $19,9 \%$ & 1,74 & ns \\
\hline sehr gut (4h und mehr Sport pro Woche) & 211 & $30,0 \%$ & 68 & $36,6 \%$ & 1,66 & ns \\
\hline gut (2-4h Sport pro Woche) & 217 & $30,9 \%$ & 60 & $32,3 \%$ & 0,36 & ns \\
\hline mäßig (1-2 h Sport pro Woche) & 70 & $10,0 \%$ & 13 & $7,0 \%$ & 1,36 & ns \\
\hline $\begin{array}{l}\text { nicht besonders (weniger als } 1 \mathrm{~h} \text { Sport } \\
\text { pro Wo.) }\end{array}$ & 24 & $3,4 \%$ & 8 & $4,3 \%$ & 0,54 & ns \\
\hline \multicolumn{7}{|l|}{ Sportarten vor der Schwangerschaft } \\
\hline keine Sportarten zusätzlich zum Reiten & 274 & $39,0 \%$ & 56 & $30,1 \%$ & 2,31 & s \\
\hline $\begin{array}{l}\text { Insgesamt sportl. aktiv zusätzlich zum } \\
\text { Reiten }\end{array}$ & 429 & $61,0 \%$ & 130 & $69,9 \%$ & 2,31 & $\mathrm{~s}$ \\
\hline Ausdauerbetonte Sportarten & 309 & $44,0 \%$ & 83 & $44,6 \%$ & 0,16 & ns \\
\hline Kraftbetonte Sportarten & 50 & $7,1 \%$ & 17 & $9,1 \%$ & 0,87 & ns \\
\hline Gymnastische Sportarten & 80 & $11,4 \%$ & 25 & $13,4 \%$ & 0,74 & ns \\
\hline Fun- und Risikosportarten & 100 & $14,2 \%$ & 42 & $22,6 \%$ & 2,50 & $\mathrm{~s}$ \\
\hline Spielsportarten & 51 & $7,3 \%$ & 10 & $5,4 \%$ & 0,98 & ns \\
\hline \multicolumn{7}{|l|}{ Leistungsstand Reiten } \\
\hline Ungeübte Reiterin & 14 & $2,0 \%$ & 3 & $1,6 \%$ & 0,36 & ns \\
\hline Geübte Reiterin & 461 & $65,6 \%$ & 134 & $72,0 \%$ & 1,73 & ns \\
\hline Leistungssportlerin im gehobenen Bereich & 202 & $28,7 \%$ & 40 & $21,5 \%$ & 2,09 & $\mathrm{~s}$ \\
\hline Berufsreiterin & 26 & $3,7 \%$ & 9 & $4,8 \%$ & 0,66 & ns \\
\hline
\end{tabular}


Reiten in der Schwangerschaft

Primiparae

Spontanentbindungen vs. sekundäre Sectiones

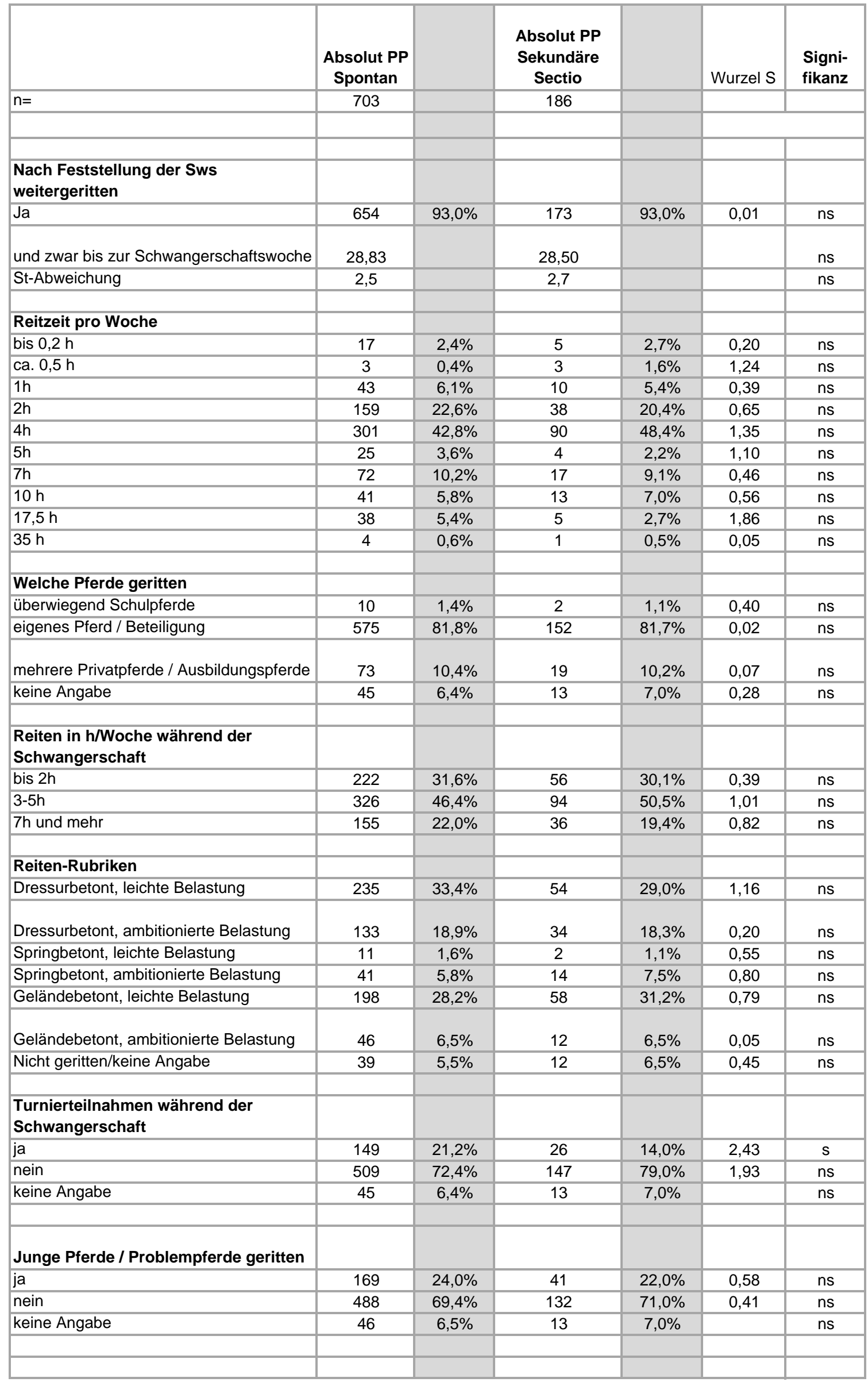


Reiten in der Schwangerschaft

Primiparae

Spontanentbindungen vs. sekundäre Sectiones

\begin{tabular}{|c|c|c|c|c|c|c|}
\hline & $\begin{array}{l}\text { Absolut PP } \\
\text { Spontan }\end{array}$ & & $\begin{array}{l}\text { Absolut PP } \\
\text { Sekundäre } \\
\text { Sectio }\end{array}$ & & Wurzel S & $\begin{array}{l}\text { Signi- } \\
\text { fikanz }\end{array}$ \\
\hline$n=$ & 703 & & 186 & & & \\
\hline \multicolumn{7}{|l|}{$\begin{array}{l}\text { Reitstil während der Schwangerschaft } \\
\text { geändert }\end{array}$} \\
\hline ja & 467 & $66,4 \%$ & 126 & $67,7 \%$ & 0,34 & ns \\
\hline nein & 190 & $27,0 \%$ & 47 & $25,3 \%$ & 0,49 & ns \\
\hline keine Angabe & 46 & $6,5 \%$ & 13 & $7,0 \%$ & & ns \\
\hline \multicolumn{7}{|l|}{$\begin{array}{l}\text { Unfall beim Reiten oder beim Umgang } \\
\text { mit dem Pferd }\end{array}$} \\
\hline ja (10 Frauen mit Mehrfachnennungen) & 64 & $9,1 \%$ & 21 & $11,3 \%$ & 0,85 & ns \\
\hline nein & 594 & $84,5 \%$ & 152 & $81,7 \%$ & 0,88 & ns \\
\hline $\begin{array}{l}\text { Kein Umgang mit Pferden während der } \\
\text { Schwangerschaft }\end{array}$ & 45 & $6,4 \%$ & 13 & $7,0 \%$ & & ns \\
\hline \multicolumn{7}{|l|}{$\begin{array}{l}\text { Trainingszustand während der } \\
\text { Schwangerschaft }\end{array}$} \\
\hline ausgezeichnet & 89 & $12,7 \%$ & 17 & $9,1 \%$ & 1,43 & ns \\
\hline sehr gut & 132 & $18,8 \%$ & 40 & $21,5 \%$ & 0,81 & ns \\
\hline gut & 244 & $34,7 \%$ & 72 & $38,7 \%$ & 1,00 & ns \\
\hline mäßig & 163 & $23,2 \%$ & 35 & $18,8 \%$ & 1,33 & ns \\
\hline nicht besonders & 75 & $10,7 \%$ & 22 & $11,8 \%$ & 0,44 & ns \\
\hline \multicolumn{7}{|l|}{$\begin{array}{l}\text { Erkrankung vor oder während der } \\
\text { Schwangerschaft }\end{array}$} \\
\hline \multicolumn{7}{|l|}{ Bluthochdruck } \\
\hline insgesamt ja & 29 & $4,1 \%$ & 20 & $10,8 \%$ & 2,77 & $\mathrm{~s}$ \\
\hline vor der Schwangerschaft & 4 & $0,6 \%$ & 1 & $0,5 \%$ & 0,05 & ns \\
\hline während der Schwangerschaft & 25 & $3,6 \%$ & 19 & $10,2 \%$ & 2,86 & $\mathrm{~s}$ \\
\hline keine Angabe & 0 & $0,0 \%$ & 0 & $0,0 \%$ & & \\
\hline \multicolumn{7}{|l|}{$\begin{array}{l}\text { Komplikationen während der } \\
\text { Schwangerschaft }\end{array}$} \\
\hline ja & 319 & $45,4 \%$ & 111 & $59,7 \%$ & 3,52 & $\mathrm{~s}$ \\
\hline nein & 384 & $54,6 \%$ & 74 & $39,8 \%$ & 3,66 & $\mathrm{~s}$ \\
\hline \multicolumn{7}{|l|}{$\begin{array}{l}\text { Komplikationen während der } \\
\text { Schwangerschaft, und zwar }\end{array}$} \\
\hline \multicolumn{7}{|l|}{ Scheiden- oder Harnwegsinfekt } \\
\hline insgesamt ja & 51 & $7,3 \%$ & 24 & $12,9 \%$ & 2,14 & $\mathrm{~s}$ \\
\hline $\begin{array}{l}\text { während der aktiven Reitzeit bis } 1 \text { Woche } \\
\text { danach }\end{array}$ & 30 & $4,3 \%$ & 20 & $10,8 \%$ & 2,71 & $\mathrm{~s}$ \\
\hline später & 21 & $3,0 \%$ & 4 & $2,2 \%$ & 0,67 & ns \\
\hline \multicolumn{7}{|l|}{ Vorzeitige Wehentätigkeit } \\
\hline insgesamt ja & 77 & $11,0 \%$ & 29 & $15,6 \%$ & 1,59 & ns \\
\hline $\begin{array}{l}\text { während der aktiven Reitzeit bis } 1 \text { Woche } \\
\text { danach }\end{array}$ & 23 & $3,3 \%$ & 10 & $5,4 \%$ & 1,18 & ns \\
\hline später & 54 & $7,7 \%$ & 19 & $10,2 \%$ & 1,04 & ns \\
\hline insgesamt nein & 239 & $34,0 \%$ & 80 & $43,0 \%$ & 2,23 & $\mathrm{~s}$ \\
\hline \multicolumn{7}{|l|}{ Vorzeitiger Blasensprung } \\
\hline insgesamt ja & 43 & $6,1 \%$ & 19 & $10,2 \%$ & 1,71 & ns \\
\hline $\begin{array}{l}\text { während der aktiven Reitzeit bis } 1 \text { Woche } \\
\text { danach }\end{array}$ & 9 & $1,3 \%$ & 6 & $3,2 \%$ & 1,43 & ns \\
\hline später & 34 & $4,8 \%$ & 13 & $7,0 \%$ & 1,06 & ns \\
\hline insgesamt nein & 273 & $38,8 \%$ & 90 & $48,4 \%$ & 2,33 & $\mathrm{~s}$ \\
\hline
\end{tabular}


Reiten in der Schwangerschaft

Primiparae

Spontanentbindungen vs. sekundäre Sectiones

\begin{tabular}{|c|c|c|c|c|c|c|}
\hline & $\begin{array}{l}\text { Absolut PP } \\
\text { Spontan }\end{array}$ & & $\begin{array}{l}\text { Absolut PP } \\
\text { Sekundäre } \\
\text { Sectio }\end{array}$ & & Wurzel S & $\begin{array}{l}\text { Signi- } \\
\text { fikanz }\end{array}$ \\
\hline$n=$ & 703 & & 186 & & & \\
\hline \multicolumn{7}{|l|}{ Zervixinsuffizienz } \\
\hline insgesamt ja & 41 & $5,8 \%$ & 12 & $6,5 \%$ & 0,31 & ns \\
\hline $\begin{array}{l}\text { während der aktiven Reitzeit bis } 1 \text { Woche } \\
\text { danach }\end{array}$ & 18 & $2,6 \%$ & 4 & $2,2 \%$ & 0,34 & ns \\
\hline später & 23 & $3,3 \%$ & 8 & $4,3 \%$ & 0,63 & ns \\
\hline insgesamt nein & 275 & $39,1 \%$ & 97 & $52,2 \%$ & 3,18 & $\mathrm{~s}$ \\
\hline \multicolumn{7}{|l|}{ Amnioninfektionssyndrom } \\
\hline insgesamt ja & 0 & $0,0 \%$ & 4 & $2,2 \%$ & 2,02 & ns \\
\hline $\begin{array}{l}\text { während der aktiven Reitzeit bis } 1 \text { Woche } \\
\text { danach }\end{array}$ & 0 & $0,0 \%$ & 0 & $0,0 \%$ & & \\
\hline später & 0 & $0,0 \%$ & 4 & $2,2 \%$ & 2,02 & ns \\
\hline \multicolumn{7}{|l|}{ Ablösung der Plazenta } \\
\hline insgesamt ja & 3 & $0,4 \%$ & 1 & $0,5 \%$ & 0,19 & ns \\
\hline $\begin{array}{l}\text { während der aktiven Reitzeit bis } 1 \text { Woche } \\
\text { danach }\end{array}$ & 0 & $0,0 \%$ & 0 & $0,0 \%$ & & \\
\hline später & 3 & $0,4 \%$ & 1 & $0,5 \%$ & 0,19 & ns \\
\hline \multicolumn{7}{|l|}{ Plazenta-Insuffizienz } \\
\hline insgesamt ja & 10 & $1,4 \%$ & 6 & $3,2 \%$ & 1,32 & ns \\
\hline $\begin{array}{l}\text { während der aktiven Reitzeit bis } 1 \text { Woche } \\
\text { danach }\end{array}$ & 4 & $0,6 \%$ & 1 & $0,5 \%$ & 0,05 & ns \\
\hline später & 6 & $0,9 \%$ & 5 & $2,7 \%$ & 1,48 & ns \\
\hline \multicolumn{7}{|l|}{ Bluthochdruck } \\
\hline insgesamt ja & 18 & $2,6 \%$ & 19 & $10,2 \%$ & 3,33 & $\mathrm{~s}$ \\
\hline $\begin{array}{l}\text { während der aktiven Reitzeit bis } 1 \text { Woche } \\
\text { danach }\end{array}$ & 6 & $0,9 \%$ & 6 & $3,2 \%$ & 1,77 & ns \\
\hline später & 12 & $1,7 \%$ & 13 & $7,0 \%$ & 2,73 & $\mathrm{~s}$ \\
\hline \multicolumn{7}{|l|}{ Präeklampsie / Gestose } \\
\hline insgesamt ja & 9 & $1,3 \%$ & 10 & $5,4 \%$ & 2,40 & $\mathrm{~s}$ \\
\hline $\begin{array}{l}\text { während der aktiven Reitzeit bis } 1 \text { Woche } \\
\text { danach }\end{array}$ & 1 & $0,1 \%$ & 1 & $0,5 \%$ & 0,71 & ns \\
\hline später & 8 & $1,1 \%$ & 9 & $4,8 \%$ & 2,28 & $\mathrm{~s}$ \\
\hline \multicolumn{7}{|l|}{ Eklampsie / HELLP-Syndrom } \\
\hline insgesamt ja & 3 & $0,4 \%$ & 6 & $3,2 \%$ & 2,12 & ns \\
\hline $\begin{array}{l}\text { während der aktiven Reitzeit bis } 1 \text { Woche } \\
\text { danach }\end{array}$ & 1 & $0,1 \%$ & 1 & $0,5 \%$ & 0,71 & ns \\
\hline später & 2 & $0,3 \%$ & 5 & $2,7 \%$ & 2,00 & ns \\
\hline \multicolumn{7}{|l|}{ Schwangerschaftsdiabetes } \\
\hline insgesamt ja & 6 & $0,9 \%$ & 3 & $1,6 \%$ & 0,77 & ns \\
\hline $\begin{array}{l}\text { während der aktiven Reitzeit bis } 1 \text { Woche } \\
\text { danach }\end{array}$ & 2 & $0,3 \%$ & 1 & $0,5 \%$ & 0,44 & ns \\
\hline später & 4 & $0,6 \%$ & 2 & $1,1 \%$ & 0,63 & ns \\
\hline \multicolumn{7}{|l|}{ Hydramnion } \\
\hline insgesamt ja & 4 & $0,6 \%$ & 1 & $0,5 \%$ & 0,05 & ns \\
\hline $\begin{array}{l}\text { während der aktiven Reitzeit bis } 1 \text { Woche } \\
\text { danach }\end{array}$ & 1 & $0,1 \%$ & 0 & $0,0 \%$ & 1,00 & ns \\
\hline später & 3 & $0,4 \%$ & 1 & $0,5 \%$ & 0,19 & ns \\
\hline & & & & & & \\
\hline & & & & & & \\
\hline
\end{tabular}


Reiten in der Schwangerschaft

Primiparae

Spontanentbindungen vs. sekundäre Sectiones

\begin{tabular}{|c|c|c|c|c|c|c|}
\hline & $\begin{array}{l}\text { Absolut PP } \\
\text { Spontan }\end{array}$ & & $\begin{array}{l}\text { Absolut PP } \\
\text { Sekundäre } \\
\text { Sectio }\end{array}$ & & Wurzel S & $\begin{array}{l}\text { Signi- } \\
\text { fikanz }\end{array}$ \\
\hline$n=$ & 703 & & 186 & & & \\
\hline \multicolumn{7}{|l|}{ Fehlbildungen } \\
\hline insgesamt ja & 1 & $0,1 \%$ & 1 & $0,5 \%$ & 0,71 & ns \\
\hline $\begin{array}{l}\text { während der aktiven Reitzeit bis } 1 \text { Woche } \\
\text { danach }\end{array}$ & 0 & $0,0 \%$ & 0 & $0,0 \%$ & & \\
\hline später & 1 & $0,1 \%$ & 1 & $0,5 \%$ & 0,71 & ns \\
\hline \multicolumn{7}{|l|}{ Uterusmyom } \\
\hline insgesamt ja & 2 & $0,3 \%$ & 5 & $2,7 \%$ & 2,00 & ns \\
\hline $\begin{array}{l}\text { während der aktiven Reitzeit bis } 1 \text { Woche } \\
\text { danach }\end{array}$ & 0 & $0,0 \%$ & 1 & $0,5 \%$ & 1,00 & ns \\
\hline später & 2 & $0,3 \%$ & 4 & $2,2 \%$ & 1,72 & ns \\
\hline \multicolumn{7}{|l|}{ Blutungen } \\
\hline insgesamt ja & 34 & $4,8 \%$ & 12 & $6,5 \%$ & 0,82 & ns \\
\hline $\begin{array}{l}\text { während der aktiven Reitzeit bis } 1 \text { Woche } \\
\text { danach }\end{array}$ & 27 & $3,8 \%$ & 10 & $5,4 \%$ & 0,85 & ns \\
\hline später & 7 & $1,0 \%$ & 2 & $1,1 \%$ & 0,09 & ns \\
\hline \multicolumn{7}{|l|}{ Fieber } \\
\hline insgesamt ja & 12 & $1,7 \%$ & 3 & $1,6 \%$ & 0,09 & ns \\
\hline $\begin{array}{l}\text { während der aktiven Reitzeit bis } 1 \text { Woche } \\
\text { danach }\end{array}$ & 6 & $0,9 \%$ & 2 & $1,1 \%$ & 0,27 & ns \\
\hline später & 6 & $0,9 \%$ & 1 & $0,5 \%$ & 0,49 & ns \\
\hline \multicolumn{7}{|l|}{ Blutgruppen-Unverträglichkeit } \\
\hline insgesamt ja & 8 & $1,1 \%$ & 5 & $2,7 \%$ & 1,24 & ns \\
\hline $\begin{array}{l}\text { während der aktiven Reitzeit bis } 1 \text { Woche } \\
\text { danach }\end{array}$ & 4 & $0,6 \%$ & 2 & $1,1 \%$ & 0,63 & ns \\
\hline später & 4 & $0,6 \%$ & 3 & $1,6 \%$ & 1,08 & ns \\
\hline \multicolumn{7}{|l|}{ Toxoplasmose } \\
\hline insgesamt ja & 1 & $0,1 \%$ & 1 & $0,5 \%$ & 0,71 & ns \\
\hline $\begin{array}{l}\text { während der aktiven Reitzeit bis } 1 \text { Woche } \\
\text { danach }\end{array}$ & 1 & $0,1 \%$ & 1 & $0,5 \%$ & 0,71 & ns \\
\hline später & 0 & $0,0 \%$ & 0 & $0,0 \%$ & & \\
\hline \multicolumn{7}{|l|}{ Sonstige Infektionskrankheiten } \\
\hline insgesamt ja & 17 & $2,4 \%$ & 8 & $4,3 \%$ & 1,18 & ns \\
\hline $\begin{array}{l}\text { während der aktiven Reitzeit bis } 1 \text { Woche } \\
\text { danach }\end{array}$ & 10 & $1,4 \%$ & 4 & $2,2 \%$ & 0,63 & ns \\
\hline später & 7 & $1,0 \%$ & 4 & $2,2 \%$ & 1,02 & ns \\
\hline \multicolumn{7}{|l|}{ Sonstige schwere Krankheiten } \\
\hline insgesamt ja & 1 & $0,1 \%$ & 0 & $0,0 \%$ & 1,00 & ns \\
\hline $\begin{array}{l}\text { während der aktiven Reitzeit bis } 1 \text { Woche } \\
\text { danach }\end{array}$ & 0 & $0,0 \%$ & 0 & $0,0 \%$ & & \\
\hline später & 1 & $0,1 \%$ & 0 & $0,0 \%$ & 1,00 & ns \\
\hline \multicolumn{7}{|l|}{ Falsche Lage des Kindes } \\
\hline insgesamt ja & 9 & $1,3 \%$ & 19 & $10,2 \%$ & 3,95 & !!! \\
\hline $\begin{array}{l}\text { während der aktiven Reitzeit bis } 1 \text { Woche } \\
\text { danach }\end{array}$ & 5 & $0,7 \%$ & 6 & $3,2 \%$ & 1,89 & !!! \\
\hline später & 4 & $0,6 \%$ & 13 & $7,0 \%$ & 3,40 & ns \\
\hline & & & & & & \\
\hline & & & & & & \\
\hline & & & & & & \\
\hline
\end{tabular}


Reiten in der Schwangerschaft

Primiparae

Spontanentbindungen vs. sekundäre Sectiones

\begin{tabular}{|c|c|c|c|c|c|c|}
\hline & $\begin{array}{l}\text { Absolut PP } \\
\text { Spontan }\end{array}$ & & $\begin{array}{l}\text { Absolut PP } \\
\text { Sekundäre } \\
\text { Sectio }\end{array}$ & & Wurzel S & $\begin{array}{l}\text { Signi- } \\
\text { fikanz }\end{array}$ \\
\hline $\mathrm{n}=$ & 703 & & 186 & & & \\
\hline \multicolumn{7}{|l|}{ Sonstiges } \\
\hline insgesamt ja & 53 & $7,5 \%$ & 13 & $7,0 \%$ & 0,26 & ns \\
\hline $\begin{array}{l}\text { während der aktiven Reitzeit bis } 1 \text { Woche } \\
\text { danach }\end{array}$ & 30 & $4,3 \%$ & 7 & $3,8 \%$ & 0,32 & ns \\
\hline später & 23 & $3,3 \%$ & 6 & $3,2 \%$ & 0,03 & ns \\
\hline \multicolumn{7}{|l|}{ ENTBINDUNG } \\
\hline \multicolumn{7}{|l|}{ Dauer der Schwangerschaft } \\
\hline Ende in Sws-Woche - Mittelwert & 39,6 & & 39,9 & & & \\
\hline Standardabweichung & 1,8 & & 2,0 & & & ns \\
\hline 25.-26. Woche & 1 & $0,1 \%$ & 0 & $0,0 \%$ & 1,00 & ns \\
\hline 27.-31. Woche & 2 & $0,3 \%$ & 1 & $0,5 \%$ & 0,44 & ns \\
\hline 32.-36. Woche & 37 & $5,3 \%$ & 14 & $7,5 \%$ & 1,07 & ns \\
\hline 37.-40. Woche & 437 & $62,2 \%$ & 86 & $46,2 \%$ & 3,90 & $\mathrm{~s}$ \\
\hline nach 40. Woche & 226 & $32,1 \%$ & 85 & $45,7 \%$ & 3,34 & $\mathrm{~s}$ \\
\hline gesamt ab 37. Woche & 663 & $94,3 \%$ & 171 & $91,9 \%$ & 1,09 & ns \\
\hline Gesamt nach 40+6 (41. SSW) & 76 & $10,8 \%$ & 34 & $18,3 \%$ & 2,44 & $\mathrm{~s}$ \\
\hline Frühgeburt bis einschließlich 36 . Woche & 40 & $5,7 \%$ & 15 & $8,1 \%$ & 1,09 & ns \\
\hline \multicolumn{7}{|l|}{ Gewicht und Größe des Kindes } \\
\hline \multicolumn{7}{|l|}{ Körpergewicht in g } \\
\hline Mittelwert in $\mathrm{g}$ & 3379 & & 3446 & & & \\
\hline Standardabweichung in $\mathrm{g}$ & 464 & & 614 & & & ns \\
\hline bis $600 \mathrm{~g}$ & 0 & $0,0 \%$ & 0 & $0,0 \%$ & & \\
\hline $601-1000 \mathrm{~g}$ & 0 & $0,0 \%$ & 0 & $0,0 \%$ & & \\
\hline $1001-1500 \mathrm{~g}$ & 1 & $0,1 \%$ & 0 & $0,0 \%$ & 1,00 & ns \\
\hline $1501-2000 \mathrm{~g}$ & 0 & $0,0 \%$ & 4 & $2,2 \%$ & 2,02 & ns \\
\hline $2001-2500 \mathrm{~g}$ & 24 & $3,4 \%$ & 12 & $6,5 \%$ & 1,58 & ns \\
\hline $2501-3000 \mathrm{~g}$ & 121 & $17,2 \%$ & 26 & $14,0 \%$ & 1,11 & ns \\
\hline $3001-3500 \mathrm{~g}$ & 300 & $42,7 \%$ & 54 & $29,0 \%$ & 3,58 & $\mathrm{~s}$ \\
\hline $3501-4000 \mathrm{~g}$ & 199 & $28,3 \%$ & 61 & $32,8 \%$ & 1,17 & ns \\
\hline $4001-4500 \mathrm{~g}$ & 50 & $7,1 \%$ & 24 & $12,9 \%$ & 2,19 & $\mathrm{~s}$ \\
\hline über $4500 \mathrm{~g}$ & 8 & $1,1 \%$ & 5 & $2,7 \%$ & 1,24 & ns \\
\hline \multicolumn{7}{|l|}{ Körpergröße in cm } \\
\hline Mittelwert & 51 & & 52 & & & \\
\hline bis $30 \mathrm{~cm}$ & 1 & $0,1 \%$ & 0 & $0,0 \%$ & 1,00 & ns \\
\hline $31-40 \mathrm{~cm}$ & 5 & $0,7 \%$ & 0 & $0,0 \%$ & 2,24 & ns \\
\hline $41-50 \mathrm{~cm}$ & 234 & $33,3 \%$ & 57 & $30,6 \%$ & 0,69 & ns \\
\hline über $50 \mathrm{~cm}$ & 461 & $65,6 \%$ & 129 & $69,4 \%$ & 0,99 & ns \\
\hline \multicolumn{7}{|l|}{ Entbindung } \\
\hline spontan & 703 & $100,0 \%$ & 0 & $0,0 \%$ & & \\
\hline geplanter Kaiserschnitt & 0 & $0,0 \%$ & 0 & $0,0 \%$ & & \\
\hline ungeplanter Kaiserschnitt & 0 & $0,0 \%$ & 186 & $100,0 \%$ & & \\
\hline Kaiserschnitt insgesamt & 0 & $0,0 \%$ & 186 & $100,0 \%$ & & \\
\hline Saugglocke oder Zange & 0 & $0,0 \%$ & 0 & $0,0 \%$ & & \\
\hline \multicolumn{7}{|l|}{ Verlauf der Entbindung } \\
\hline ohne Schwierigkeiten & 503 & $71,6 \%$ & 29 & $15,6 \%$ & 17,72 & $\mathrm{~s}$ \\
\hline mit Schwierigkeiten & 155 & $22,0 \%$ & 125 & $67,2 \%$ & 11,94 & s \\
\hline $\begin{array}{l}\text { Verlängerter Geburtsverlauf oder Geb.- } \\
\text { stillstand }\end{array}$ & 83 & $11,8 \%$ & 71 & $38,2 \%$ & 7,00 & $\mathrm{~s}$ \\
\hline $\begin{array}{l}\text { Freitext-Einträge zu schwierigen } \\
\text { Verläufen }\end{array}$ & 167 & $23,8 \%$ & 128 & $68,8 \%$ & 11,99 & $\mathrm{~s}$ \\
\hline
\end{tabular}


Reiten in der Schwangerschaft

Primiparae

Spontanentbindungen vs. sekundäre Sectiones

\begin{tabular}{|c|c|c|c|c|c|c|}
\hline & $\begin{array}{l}\text { Absolut PP } \\
\text { Spontan }\end{array}$ & & $\begin{array}{l}\text { Absolut PP } \\
\text { Sekundäre } \\
\text { Sectio }\end{array}$ & & Wurzel S & $\begin{array}{l}\text { Signi- } \\
\text { fikanz }\end{array}$ \\
\hline$n=$ & 703 & & 186 & & & \\
\hline Kindslage - Angaben insgesamt & 517 & $73,5 \%$ & 134 & $72,0 \%$ & 0,41 & ns \\
\hline unbekannt & 186 & $26,5 \%$ & 52 & $28,0 \%$ & 0,41 & ns \\
\hline $\begin{array}{l}\text { Normale Schädellage (\% aller bekannten } \\
\text { Kindslagen) }\end{array}$ & 500 & $96,7 \%$ & 96 & $71,6 \%$ & 7,43 & $\mathrm{~s}$ \\
\hline $\begin{array}{l}\text { Komplizierte Schädellage (\% aller } \\
\text { bekannten Kindslagen) }\end{array}$ & 9 & $1,7 \%$ & 23 & $17,2 \%$ & 5,49 & $\mathrm{~s}$ \\
\hline $\begin{array}{l}\text { Beckenendlage (\% aller bekannten } \\
\text { Kindslagen) }\end{array}$ & 7 & $1,4 \%$ & 11 & $8,2 \%$ & 3,33 & s \\
\hline $\begin{array}{l}\text { Quer- oder Schräglage (\% aller } \\
\text { bekannten Kindslagen) }\end{array}$ & 1 & $0,2 \%$ & 4 & $3,0 \%$ & 2,22 & ns \\
\hline \multicolumn{7}{|l|}{ Kind nach der Geburt } \\
\hline gesund geboren & 669 & $95,2 \%$ & 174 & $93,5 \%$ & 0,82 & ns \\
\hline krank geboren & 34 & $4,8 \%$ & 12 & $6,5 \%$ & 0,82 & ns \\
\hline Totgeburt & 0 & $0,0 \%$ & 0 & $0,0 \%$ & & \\
\hline Freitext-Einträge & 33 & $4,7 \%$ & 12 & $6,5 \%$ & 0,89 & ns \\
\hline \multicolumn{7}{|l|}{ Kind (er) nach einem Jahr } \\
\hline Kind (alle Kinder) gesund & 688 & $97,9 \%$ & 181 & $97,3 \%$ & 0,42 & ns \\
\hline Kind (bzw. eines der Kinder) krank & 13 & $1,8 \%$ & 5 & $2,7 \%$ & 0,65 & ns \\
\hline Kind (bzw. eines der Kinder) verstorben & 1 & $0,1 \%$ & 0 & $0,0 \%$ & 1,00 & ns \\
\hline Freitext-Einträge & 12 & $1,7 \%$ & 4 & $2,2 \%$ & 0,38 & ns \\
\hline
\end{tabular}




\begin{tabular}{|c|c|c|c|c|c|c|c|}
\hline & & $\begin{array}{l}\text { Ohne } \\
\text { Unfälle } \\
\text { beim } \\
\text { Reiten oder } \\
\text { beim } \\
\text { Umgang } \\
\text { mit dem } \\
\text { Pferd }\end{array}$ & & $\begin{array}{c}\text { Mit } \\
\text { Unfällen } \\
\text { beim } \\
\text { Reiten oder } \\
\text { beim } \\
\text { Umgang } \\
\text { mit dem } \\
\text { Pferd }\end{array}$ & & & $\begin{array}{l}\text { Odds- } \\
\text { Ratio }\end{array}$ \\
\hline & $n=$ & 1407 & & 151 & & & \\
\hline & & & & & & \multicolumn{2}{|c|}{ Wurzel S } \\
\hline Unfallfolgen & der Schwangerschaft & 12 & $0,9 \%$ & 15 & $9,9 \%$ & 3,71 & 12,82 \\
\hline Unfallfolgen & $\begin{array}{l}\text { Aus einem Unfall vor der } \\
\text { Schwangerschaft }\end{array}$ & 28 & $2,0 \%$ & 17 & $11,3 \%$ & 3,57 & 6,25 \\
\hline Zwang zu schnellen Entscheidungen & $\begin{array}{l}\text { kam vor, Ich fühlte mich } \\
\text { dadurch stark belastet. }\end{array}$ & 54 & $3,8 \%$ & 20 & $13,2 \%$ & 3,35 & 3,83 \\
\hline Unfallrisiko & $\begin{array}{l}\text { teilweise Reiten mit } \\
\text { gewissem Risiko }\end{array}$ & 66 & $4,7 \%$ & 22 & $14,6 \%$ & 3,38 & 3,47 \\
\hline Junge Pferde / Problempferde geritten & ja & 302 & $21,5 \%$ & 71 & $47,0 \%$ & 6,08 & 3,25 \\
\hline Reiten während der Schwangerschaft & $\begin{array}{l}\text { Spitzensport Dressur, } \\
\text { Springen, Gelände }\end{array}$ & 35 & $2,5 \%$ & 10 & $6,6 \%$ & 2,00 & 2,78 \\
\hline Reitzeit pro Woche & $17,5 \mathrm{~h}$ & 59 & $4,2 \%$ & 15 & $9,9 \%$ & 2,30 & 2,52 \\
\hline Bluthochdruck während der SWS & insgesamt ja & 49 & $3,5 \%$ & 12 & $7,9 \%$ & 1,98 & 2,39 \\
\hline Reiterliche Disziplinen während der Sws & $\begin{array}{l}\text { Springen mittlere } \\
\text { Belastung }\end{array}$ & 63 & $4,5 \%$ & 15 & $9,9 \%$ & 2,19 & 2,35 \\
\hline Starke Konzentration kam vor & $\begin{array}{l}\text { Ich fühlte mich dadurch } \\
\text { stark belastet. }\end{array}$ & 66 & $4,7 \%$ & 15 & $9,9 \%$ & 2,10 & 2,24 \\
\hline Reiterliche Disziplinen während der Sws & $\begin{array}{l}\text { Springen Mittel, Leistung } \\
\text { plus Spitzensport }\end{array}$ & 115 & $8,2 \%$ & 25 & $16,6 \%$ & 2,69 & 2,23 \\
\hline Welche Pferde geritten & $\begin{array}{l}\text { mehrere Privatpferde / } \\
\text { Ausbildungspferde }\end{array}$ & 131 & $9,3 \%$ & 28 & $18,5 \%$ & 2,84 & 2,22 \\
\hline Reiterliche Belastung vor der SWS & $\begin{array}{l}\text { Spitzensport alle } \\
\text { Reitsportarten }\end{array}$ & 90 & $29,8 \%$ & 19 & $26,8 \%$ & 0,80 & 2,11 \\
\hline Reiten-Rubriken & $\begin{array}{l}\text { Springbetont, ambitionierte } \\
\text { Belastung }\end{array}$ & 73 & $5,2 \%$ & 15 & $9,9 \%$ & 1,89 & 2,02 \\
\hline Distanzreiten & insgesamt geritten & 105 & $7,5 \%$ & 21 & $13,9 \%$ & 2,22 & 2,00 \\
\hline Trotz Warnzeichen weitergeritten ... & ja & 84 & $6,0 \%$ & 17 & $11,3 \%$ & 2,00 & 2,00 \\
\hline $\begin{array}{l}\text { Sportlicher Trainingszustand vor der } \\
\text { Schwangerschaft }\end{array}$ & $\begin{array}{l}\text { ausgezeichnet (täglich } \\
\text { mind. } 1 \mathrm{~h} \text { Sport) }\end{array}$ & 300 & $21,3 \%$ & 51 & $33,8 \%$ & 3,11 & 1,88 \\
\hline Reiten während der Schwangerschaft & $\begin{array}{l}\text { Ausreiten insgesamt } \\
\text { geritten }\end{array}$ & 1151 & $81,8 \%$ & 135 & $89,4 \%$ & 2,81 & 1,88 \\
\hline $\begin{array}{l}\text { oder anderes in der Schwangerschaft } \\
\text { belastet: }\end{array}$ & $\begin{array}{l}\text { Sehr stark und stark } \\
\text { belastet }\end{array}$ & 319 & $22,7 \%$ & 53 & $35,1 \%$ & 3,08 & 1,84 \\
\hline $\begin{array}{l}\text { Trainingszustand während der } \\
\text { Schwangerschaft }\end{array}$ & ausgezeichnet & 153 & $10,9 \%$ & 27 & $17,9 \%$ & 2,17 & 1,78 \\
\hline $\begin{array}{l}\text { Durchschnittliche Reitdauer/Tag während der } \\
\text { SWS }\end{array}$ & $2-3 \mathrm{~h}$ & 205 & $14,6 \%$ & 35 & $23,2 \%$ & 2,42 & 1,77 \\
\hline Ich habe mit dem Reiten aufgehört, & andere Gründe & 230 & $16,3 \%$ & 38 & $25,2 \%$ & 2,41 & 1,72 \\
\hline $\begin{array}{l}\text { Turnierteilnahmen während der } \\
\text { Schwangerschaft }\end{array}$ & ja & 262 & $18,6 \%$ & 42 & $27,8 \%$ & 2,42 & 1,68 \\
\hline Frühere Kompl.: & Summe der Nennungen & 469 & $33,3 \%$ & 69 & $45,7 \%$ & 2,91 & 1,68 \\
\hline
\end{tabular}




\begin{tabular}{|c|c|c|c|c|c|c|c|}
\hline & & $\begin{array}{c}\text { Ohne } \\
\text { Unfälle } \\
\text { beim } \\
\text { Reiten oder } \\
\text { beim } \\
\text { Umgang } \\
\text { mit dem } \\
\text { Pferd }\end{array}$ & & $\begin{array}{c}\text { Mit } \\
\text { Unfällen } \\
\text { beim } \\
\text { Reiten oder } \\
\text { beim } \\
\text { Umgang } \\
\text { mit dem } \\
\text { Pferd }\end{array}$ & & & $\begin{array}{l}\text { Odds- } \\
\text { Ratio }\end{array}$ \\
\hline & $n=$ & 1407 & & 151 & & & \\
\hline & & & & & & \multicolumn{2}{|l|}{ Wurzel S } \\
\hline $\begin{array}{l}\text { oder anderes in der Schwangerschaft } \\
\text { belastet: }\end{array}$ & stark & 247 & $17,6 \%$ & 39 & $25,8 \%$ & 2,23 & 1,64 \\
\hline Unfallrisiko & $\begin{array}{l}\text { fühlte mich einigermaßen } \\
\text { sicher, Unfallrisiko gering }\end{array}$ & 550 & $39,1 \%$ & 77 & $51,0 \%$ & 2,79 & 1,62 \\
\hline Reittage pro Woche vor der SWS & täglich & 472 & $33,5 \%$ & 67 & $44,4 \%$ & 2,56 & 1,58 \\
\hline $\begin{array}{l}\text { Reiten in } \mathrm{h} / \text { Woche während der } \\
\text { Schwangerschaft }\end{array}$ & 7h und mehr & 285 & $20,3 \%$ & 43 & $28,5 \%$ & 2,15 & 1,57 \\
\hline Reiterliche Disziplinen während der Sws & $\begin{array}{l}\text { Springen insgesamt } \\
\text { geritten }\end{array}$ & 251 & $17,8 \%$ & 38 & $25,2 \%$ & 1,99 & 1,55 \\
\hline Reittage pro Woche während der SWS & täglich & 206 & $14,6 \%$ & 31 & $20,5 \%$ & 1,72 & 1,51 \\
\hline Reittage pro Woche während der SWS & $3 x /$ Wo oder mehr & 860 & $74,7 \%$ & 105 & $77,8 \%$ & 0,86 & 1,45 \\
\hline $\begin{array}{l}\text { Ich fühlte mich durch Arbeit, Beruf, Familie } \\
\text { oder anderes in der Schwangerschaft } \\
\text { belastet: }\end{array}$ & $\begin{array}{l}\text { sehr häufig und häufig } \\
\text { belastet }\end{array}$ & 350 & $24,9 \%$ & 49 & $32,5 \%$ & 1,90 & 1,45 \\
\hline $\begin{array}{l}\text { Ich fühlte mich durch Arbeit, Beruf, Familie } \\
\text { oder anderes in der Schwangerschaft } \\
\text { belastet: }\end{array}$ & selten & 401 & $28,5 \%$ & 32 & $21,2 \%$ & 2,07 & 0,67 \\
\hline Reittage pro Woche während der SWS & 2x pro Woche & 392 & $27,9 \%$ & 31 & $20,5 \%$ & 2,10 & 0,67 \\
\hline $\begin{array}{l}\text { Reiten in } \mathrm{h} / \text { Woche während der } \\
\text { Schwangerschaft }\end{array}$ & bis $2 \mathrm{~h}$ & 483 & $34,3 \%$ & 38 & $25,2 \%$ & 2,44 & 0,64 \\
\hline $\begin{array}{l}\text { Sportlicher Trainingszustand vor der } \\
\text { Schwangerschaft }\end{array}$ & $\begin{array}{l}\text { sehr gut (4h und mehr } \\
\text { Sport pro Woche) }\end{array}$ & 410 & $29,1 \%$ & 31 & $20,5 \%$ & 2,46 & 0,63 \\
\hline $\begin{array}{l}\text { Ich fühlte mich durch Arbeit, Beruf, Familie } \\
\text { oder anderes in der Schwangerschaft } \\
\text { belastet: }\end{array}$ & kaum & 359 & $25,5 \%$ & 26 & $17,2 \%$ & 2,53 & 0,61 \\
\hline Reittage pro Woche vor der SWS & $2 x$ pro Woche & 191 & $13,6 \%$ & 13 & $8,6 \%$ & 2,02 & 0,60 \\
\hline $\begin{array}{l}\text { Durchschnittliche Reitdauer/Tag während der } \\
\text { SWS }\end{array}$ & $1 \mathrm{~h}$ & 1187 & $84,4 \%$ & 115 & $76,2 \%$ & 2,28 & 0,59 \\
\hline Unfallrisiko & $\begin{array}{l}\text { fühlte mich völlig sicher, } \\
\text { Unfall ausgeschlossen }\end{array}$ & 673 & $47,8 \%$ & 50 & $33,1 \%$ & 3,63 & 0,54 \\
\hline Junge Pferde / Problempferde geritten & nein & 996 & $70,8 \%$ & 79 & $52,3 \%$ & 4,35 & 0,45 \\
\hline Nach Feststellung der Sws weitergeritten & Ja & 1292 & $91,8 \%$ & 151 & $100,0 \%$ & 11,19 & 0,18 \\
\hline Dauer der Schwangerschaft & $\begin{array}{l}\text { Ende in Sws-Woche - } \\
\text { Mittelwert }\end{array}$ & 39,62118 & & 39,54967 & & & \\
\hline
\end{tabular}

